\title{
Quality assurance in clinical trials in breast cancer
}

Citation for published version (APA):

Poortmans, P. M. (2005). Quality assurance in clinical trials in breast cancer. [Doctoral Thesis, Maastricht University]. https://doi.org/10.26481/dis.20050610pp

Document status and date:

Published: 01/01/2005

DOI:

10.26481/dis.20050610pp

Document Version:

Publisher's PDF, also known as Version of record

\section{Please check the document version of this publication:}

- A submitted manuscript is the version of the article upon submission and before peer-review. There can be important differences between the submitted version and the official published version of record.

People interested in the research are advised to contact the author for the final version of the publication, or visit the DOI to the publisher's website.

- The final author version and the galley proof are versions of the publication after peer review.

- The final published version features the final layout of the paper including the volume, issue and page numbers.

Link to publication

\footnotetext{
General rights rights.

- You may freely distribute the URL identifying the publication in the public portal. please follow below link for the End User Agreement:

www.umlib.nl/taverne-license

Take down policy

If you believe that this document breaches copyright please contact us at:

repository@maastrichtuniversity.nl

providing details and we will investigate your claim.
}

Copyright and moral rights for the publications made accessible in the public portal are retained by the authors and/or other copyright owners and it is a condition of accessing publications that users recognise and abide by the legal requirements associated with these

- Users may download and print one copy of any publication from the public portal for the purpose of private study or research.

- You may not further distribute the material or use it for any profit-making activity or commercial gain

If the publication is distributed under the terms of Article $25 \mathrm{fa}$ of the Dutch Copyright Act, indicated by the "Taverne" license above, 
Quality assurance in clinical trials in breast cancer

PHILIP POORTMANS 
ISBN $90-9019524-6$

Copyright 22005 Phillip Poortmans, Tilburg

All rights reserved. No parts of this publication may be reproduced, stored in a retrieval system, or transmirted in any form or by any means, elcctronically, mechanically, by photocopying or otherwise, without the prior permission of the author.

Onslag: Cees van Dun

Vomgexing en druk: Drukkerij Bulckens NV, Herentals 


\section{Quality assurance in clinical trials in breast cancer}

Kwaliteitsborging bij klinisch wetenschappelijk onderzoek

bij borstkanker

\section{PROEFSCHRIFT}

ter verkrijging van de graad van doctor

aan de Universiteit Maastricht,

op gezag van de Rector Magnificus,

Prof. mr. G.P.M.F. Mols,

volgens het besluit van het College van Decanen,

in het openbaar te verdedigen

op vrijdag 10 juni 2005 om 16.00 uur.

door

Philip Martin Peter Poortmans 


\section{Promotores:}

Prof. dr. Ph. Lambin

Prof. dr. W. Van den Bogaert, UZ-KULeuven, België

\section{Copromotor:}

Dr. sc. J.B. Davis, UH Zürich, Switserland

\section{Beoordelingscommissie:}

Prof. dr. M.F. von Meyenfeldt (voorzitter)

Dr. ir. A. Minken

Dr. D. de Ruysscher

Prof. dr. P. Scalliet, UCL St. Luc, Bruxelles, België

Prof. dr. H.C. Schouten

The work described in this thesis has been carried out at the department of radiocherapy of the Dr. Bernard Verbecten Institute, "Tilburg, The Netherlands, largely on behalf of the EORTC Radiotherapy Group. The Dr. Bernard Verbecten Institute, AstraZeneca B.V., CMS GmbH, Nucletron B.V., Novartis Pharma B.V., Siemens Nederland N.V. Medical Solutions, GlaxoSmithKline, Mundipharma Pharmaceuticals B.V., Nycomed Christiaens B.V. and Roche Nederland B.V. are gratefully acknowledged for the financial contribution to the costs of the publication of this thesis. 
The patient is the centre

of the medical universe

around which all our work revolves and towards which all our efforts tend.

J.B. Murphy, $1857-1916$ 


\section{PART I: INTRODUCTION}

Chapter 1 General introduction

Chapter 2 The quality assurance programme of the Radiotherapy Group of the European

Organization for Research and Treament of Cancer (EORTC): a cricical appraisal of 20 years of continuous efforts.

\section{PART II: EORTC TRIAL 22881/10882}

Chapter 3 Recurrence rates after treatment of breast cancer with standard radiotherapy with or without additional radiation.

Chapter 4 The influence of the boost technique on local control in breast conserving treatment in the EORTC "boost versus no boost" randomised trial.

Chapter 5 Quality Assurance in the EORTC phase III randomised 'boost vs, no boost' trial for breast conserving therapy: comparison of the results of two individual case reviews performed early and late during the accrual period.

\section{Part III: EORTC TRIAL 22922/10925}

Chapter 6 The potential impact of treatment variations on the results of radiotherapy of the internal mammary lymph node chain: A quality-assurance report on the dummy run of EORTC phase III randomized trial $22922 / 10925$ in stage I-III breast cancer.

Chapter 7 A glance on qualiry assurance in EORTC study 22922 evaluating techniques for internal mammary and mediall supraclavicular lymph node chain irradiation in breast cancer.

Chapter 8 Quality assurance of EORTC trial 22922/10925 investigating the role of internal mammary - medial supraclavicular irradiation in stage I-III breast cancer: the individual case review.

Chapter 9 Quality assurance in the EORTC randomised trial 22922/10925 investigating irradiation of the internal mammary and medial stupraciavicular lymph node chain.

\section{PART TV: SUMMARY}

Chapter 10 The Quality Assurance Programme of the Radiotherapy Group of the European Organisation for Research and Treament of Cancer: Past, Present and Future.

Chapter 11 Summary and future prospects

\section{Samenvatting}




\section{CHAPTER 1}

Quality Assurance in clinical trials in breast cancer:

General introduction. 
Breast cancer is the most common cancer in women in the western industrialised world with an incidence of $124 / 100,000$ women per year in the Netherlands in 2000 . This brings the lifetime risk for breast cancer to 1 out of every 9 women. Among the explanations for the increasing incidence (from 100.6 in 1989 to an expected 159.9 in 2015 in The Netherlands) are the rising life expectancy with a higher risk of breast cancer in elderly women and also the marked change in family planning, assisted by the use of contraceptive measures, with a decreasing birth rate and an increasing age at giving birth. Breast cancer mortality rates in The Netherlands decreased gradually from 39.3/100,000 women per year in 1989 to $33.8 / 100,000$ women per year in 2000. The 2 most plausible explanations for the improved breast cancer survival are early detection thanks to public awareness and mass screening on one hand and an improvement in the results of breast cancer treatment. Moreover, the patient and tumour characteristics of the breast cancer population might have changed over time as well.

Especially in the treatment of breast cancer the approach is in most cases multidisciplinary with a close co-operation between surgeons, radiation oncologists and medical oncologists. The possible combinations of the different elements of the multimodality approach are described in current guidelines that exist at the national and the international level. Surgery and Radiotherapy are combined to optimise loco-regional control and to safeguard the aesthetic aspects of treatment and the body image of the patient with the use of a breast conserving approach when feasible. The debate on the influence of loco-regional tumour control on breast cancer and overall survival will probably only be closed after the final analysis of large prospective multi-centre trials like the European Organisation for Research and Treatment of Cancer (EORTC) No. $22922 / 10925$ trial investigating the value of adjuvant Radiotherapy of the regional lymph nodes. Adjuvant systemic treatment, including chemotherapy and hormonal treatments, mainly focuses on the eradication of microscopical metastatic disease in highrisk patients. Hormonal treatment will, in contrast to chemotherapy, decrease to a moderate extent the local failure rate after surgery alone or surgery combined with Radiotherapy. The indications have been refined and especially broadened over the years such that nowadays only the very low risk patients will receive no adjuvant systemic treatment. Recent developments in gene and protein analysis might soon enable us to predict more accurately the risk of local and systemic recurrences and survival. Patients with a predicted low-risk might than be spared unnecessary systemic treatments while patients with a high-risk profile could possibly benefit from intensified treatment regimens. With the continuing increase in long-term survival, the interest of Quality Assurance for all aspects of the treatment will grow to optimise the balance between 
efficacy and side effects.

A large part of the progress has been made with the help of well-designed prospective studies accruing thousands of breast cancer patients. Thanks to this large numbers, several meta-analyses examining a number of important questions could be performed. Some of them are repeated every 5 years to evaluate the long-term effect of treatments. However, some questions remain unanswered, several of them concerning Radiotherapy: The EORTC Radiotherapy and Breast Groups have conducted several large prospective randomised multi-centre trials focussing on several aspects of the treatment of breast cancer patients. One of them, the EORTC trial No. 10801, is considered as a milestone in the local treatment of breast cancer by confirming that breast conserving therapy and modified radical mastectomy give the same outcome in local control, disease-free survival. and survival for breast cancer patients with tumours up to $5 \mathrm{~cm}$. These data have been recently pooled with a similar Danish trial, confirming the results after a median followup of 13.4 years. In this trial however it became clear that the treatment results varied very significantly from one participating institute to another and this for both randomisation arms (Figure 1). This finding has brought the issue of the quality of the treatment into the light of day and has increased the interest in quality control what changed over time in Quality Assurance. The history of Quality Assurance in the EORTC Radiotherapy Group, a real pioneer in this field, is described in chapter 2 of this thesis.

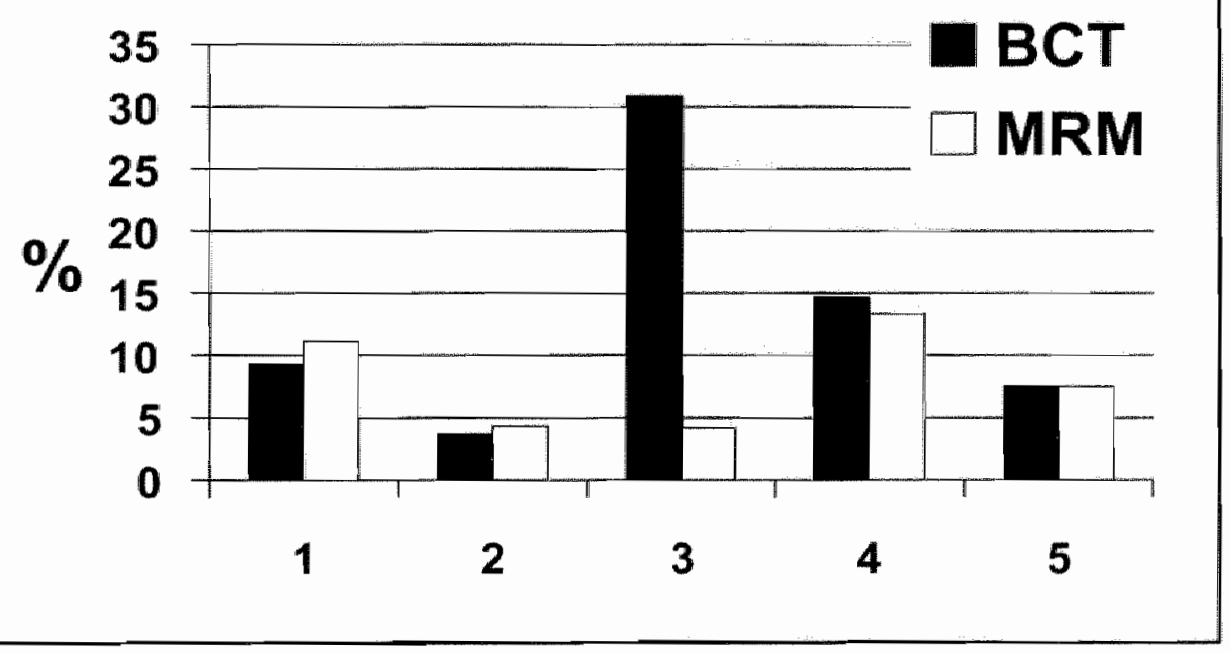

Figure 1. Local recurrence nates for the 5 major participating institutes in EORTC trial No. 10801. $B C T=$ breast conserving therapy; $M R M=$ modified radical mastectomy. 
The consecutive trial of the EORTC Radiotherapy and Breast Groups, No. $22881 / 10882$, investigating local treatment in breast cancer included 5569 patients treated with breast conserving therapy. This was one of the first trials for which a comprehensive Quality Assurance programme was organised. It was co-ordinated by Geertjan van Tienhoven and has led to several publications and helped in the development of guidelines for Quality Assurance in Radiotherapy. In contrast to the Trial No. 10801 and probably at least in part as a consequence of all the efforts to optimise the quality of treatment, we found no difference in local recurrence rates between the participating institutes (Figure 2). One of the aspects of Quality Assurance in this trial which was never looked at and never analysed carefully, was the comparison of an individual case review performed early and late during the course of the trial to see whether Quality Assurance by itself had a positive influence on the compliance to the protocol guidelines. Chapters 3,4 and 5 report on the primary results, the analysis of the boost treatment techniques and the comparison of the early and late individual case reviews of this trial respectively.

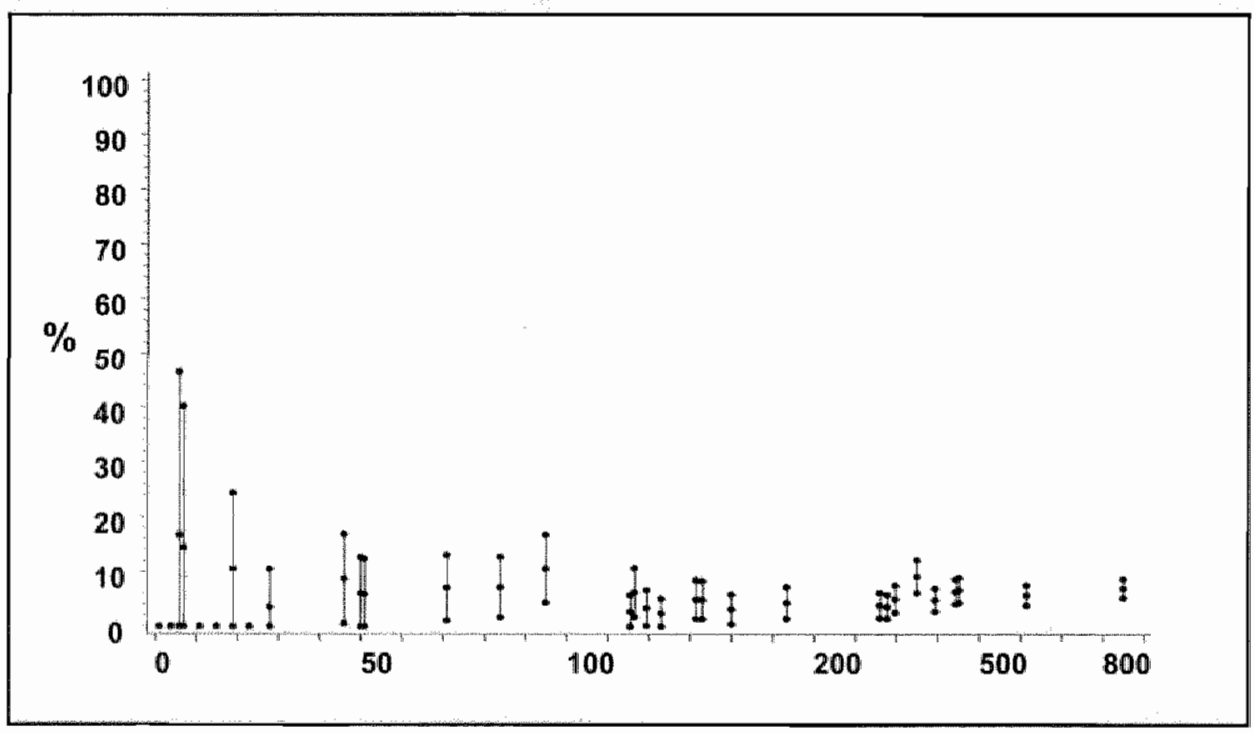

Figure 2. Local recurrence vates for all participating institutes in EORTC trial

No. 22881/10882. Numbers on abscissa are the number of randonised patients per institute.

Until the seventies, the treatment approach to breast cancer consisted of the addition of radical surgery, loco-regional Radiotherapy and on top of this systemic therapy. In the eighties however, it became clear that an aggressive treatment approach might not be appropriate for low risk patients and that this might even lead to an increased risk of complications consequently having a negative influence on survival. This has led to a 
generally more selective use of the different treatment modalicies and in some institures even to a reluctance to the use of Radiotherapy, sometimes even in high-risk patients. This led to lengthy debates and to local treatment guidelines, which were often based more on beliefs and habits rather than on scientific evidence. When the EORTC Radiotherapy and Breast Groups, in 1992, launched a joint initiative for a trial investigating the value of regional Radiotherapy in stage I to III breast cancer patients, at first the Protocol Review Committee of the EORTC did not support the study for lack of projected benefit (although this was larger than in most studies on systemic therapy), but in 1996 approval was given. However, when 1 year later, in 1997, 2 very important papers on this topic appeared in the New England Journal of Medicine, showing survival benefit in randomised studies after post-operative irradiation, the debate on the value of the trial was reopened. Finally, the debate re-enforced the idea that the question asked in the trial was a valid one and not yet answered by the aforementioned publications. This explains why, after a short hesitation, the parient accrual even rose with an increasing number of participating institutes. Similar to the preceding EORTC trial, a very comprehensive Quality Assurance programme was organised, now co-ordinared by the author of this thesis. Chapters 6 to 9 describe this work.

Quality Assurance has been progressively recognised as an important part of clinical trial research, as well as in daily cancer treatment. For many years now, the EORTC has already a Quality Assurance Committee with members from most disciplines involved in cancer research. Radiotherapy has always played a leading role in this. This year, a special issue of the European Journal of Surgical Oncology will appear with a contribution on Quality Assurance from every major discipline in oncology. Chapter 10 deals with the Radiotherapy contribution.

In summary, this thesis focuses on various aspects of Quality Assurance in clinical trials, especially in breast cancer. The results of one of the involved large prospective multicentre trials are included as an example of the high walue of this kind of research in terms of "evidence based medicine". 


\section{CHAPTER 2}

The Quality Assurance programme of the Radiotherapy Group of the European Organization for Research and Treatment of Cancer (EORTC): a critical appraisal of 20 years of continuous efforts.

VE. Kouloulias, P.M. Poortmans, J. Bernier, J.-C. Horiot, K.-A. Johansson, B. Davis, F, Godson, G. Garavaglia, M. Pierart, E. van der Schueren, for the European Organization for Research and Treatment of Cancer Radiotherapy Group.

European Journal of Cancer 39: 430-437, 2003. 


\section{Abstract}

In 1982, the European Organization for Research and Treatment of Cancer (EORTC) Radiotherapy Group established the Quality Assurance (QA) programme. During the past 20 years. QA procedures have become a major part of the activities of the group. The methodology and steps of the QA programme over the past 20 years are briefly described. Probletrs and conclusions arising from the results of the long-lasting QA programme in the EORTC radiotherapy group are discussed and emphasised. The EORTC radotherapy group continues to lead QA in the European radiotherapy community. Furure challenges and perspectives are proposed.

Keywords: Quality assurance; Radiotherapy; European Organization for Research and Treatment of Cancer (EORTC); Chnical trials; Review 


\section{Introduction}

In a period where an overhaul of costly healthcare systems is at the centre of looming budget battles in most industrialised countries, the deleterious consequences of poor quality treatments undoubtedly contribute to the rise of health costs. Moreover, it is now well documented that large national differences in survival rates are found among patients with similar diagnostic backgrounds: variations in care quality not only affecr the effectiveness of therapeutic management for a given disease, but also lead to severe complications which significantly reduce the quality of life. In this perspective, the European Organization for Research and Treatment of Cancer (EORTC) Radiotherapy Group developed, throughout the least 20 years, a comprehensive Quality Assurance (QA) programme as a means to develop cost-effective medical practice.

As a rule, this QA programme was implemented in every clinical trial activated by the Radiotherapy Group and has resulted in a specific field of clinical research to develop methodologies that are now gradually transferred from clinical research entities to the entire radiotherapy community.

The objectives of this retrospective analysis are threefold: (1) To revisit the key issues addressed by the cohort of quality control procedures set-up over the last 20 years, by the Radiotherapy Group; (2) To analyse the potential impact of their message(s) on disease outcome following radiotherapy; (3) To show how the experience gained over the last two decades can be used to build a platform of novel approaches in QA.

\section{Quality Assurance in radiotherapy: from strategies to implementation}

Since 1982, the year of the activation of its Quality Assurance programme, the Radiotherapy Group progressively extended its original project into pilot studies in an attempt (a) to promote a systematic check of individual patients and (b) to improve the reliability of treatment procedures. Once general requirements needed to warrant a valid cooperation were identified, the group directed its efforts to the clinical ground and, in particular, to the setup of reliable control procedures to improve the quality of protocols of phase III clinical studies. This general philosophy led to considerable improvements in the writing of protocols, in data management and also in the detection and correction of dosimetric parameters.

The QA programme was actually developed in three main phases articulated around the development of two Quality Systems: the Programme of Physics Audir Quality and the Assurance of Protocol Compliance. In the first phase, that roughly lasted 5 years 
(1982-1987), various centres were visited by a team of radiotherapists and radiation physicists. In 1987, a vast programme of mailed dosimetry was activated to document, through a large number of beam calibrations and measurements, the profile of the dose deviations between the values determined by the team of radio-physicists of the EORTC and those reported by institutions. In 1987, the Radiotherapy Group activated a second phase of the QA programme and set up a series of procedures (dummy-runs) to document systematic errors made in single institutions and control the accuracy of the design and the application of phase III study protocols. Finally, in 1989, a third phase, more patient-oriented, was activated to tackle random errors: individual case reviews directed to patient data and treatment parameters were aimed at improving the compliance of the participating centres to study protocols and at detecting obscurities in protocol guidelines.

All this implementation of QA procedures was consolidated during the 1995-1996 period, which was actually characterised by the further development of QA procedures that had been validated during the three phases mentioned above, with a particular emphasis put on the mailed TLD programme for the Programme of Physics Audit Quality, and on dummy-runs and individual case reviews for the Programme for Assurance of Protocol Compliance.

The procedure of QA is defined most generally as all those planned and systematic actions necessary to provide adequate confidence that a product or service will satisfy given requirements for quality. QA in radiotherapy [1] is defined by all those procedures that ensure consistency of the radiotherapy prescription and the safe fulfilment of that prescription, with regard to the dose to the target volume, together with a minimal dose to normal rissues, minimal exposure of personnel and adequate patient monitoring aimed at determining the end result of treatment. Quality Standards are a criterion or ser of criteria against which the quality of any form of activity can be assessed. A Quality Audit is a systematic and independent review (examination and evaluation) of the complete treatment delivery, to determine whether the system is implemented effectively and whether the activities and results produce the required end-point complying with the predetermined quality standards.

The sequence of published actions concerning QA is presented in chronological order and divided into two sections: those related to department-structure and radio-physics and those related to clinical trials. 


\section{Historical review}

\subsection{Department structure and physics}

The first QA activities in the EORTC Radiotherapy Group were reported in 1986. The evaluation included three steps: a comparison of megavoltage units, technical and staff environment, and data present in clinical and radiotherapy charts for each centre; radiation physics calibration of photon and electron beams; and radiation physics measurements on an anatomical phantom. The study included 17 institutions that were visited by a group of 'experts' in radiotherapy and radiation physics from January 1982 to December 1984 . Horiot and colleagues [2] reported the first part of the QA study in terms of assessment of medical staff and equipment. Large variations were observed in the number of patients treated per year, per radiation oncologist, per radiation physicist and per rechnologist. The number of simulators was sub-optimal in $12 / 17$ centres. In $5 / 17$ centres, major problems were present which made it difficult to comply with all of the requirements of the EORTC protocols. However, the quality of work-up regarding tumour extension was considered to be satisfactory in all centres. The second part was reported by Johansson and colleagues [3] regarding dosimetric intercomparisons. The deviations between the absorbed dose values, for specific points along the beam axis in a water phantom, determined by the QA committee and reported by the hospitals were within an acceptable level of variation $( \pm 3 \%)$. In some scanning electron beams, large deviations were found. Concerning the flatness and symmetry of the beams, $73 \%$ of the $\mathrm{X}$-ray and $60 \%$ of the electron beams were within the acceptable levels of variations. The third part was again reported by Johansson and colleagues [4] regarding intercomparison in an anatomical phantom. A tonsillar tumour and a homolateral subdigastric node were marked in an anatomical phantom. The institutions were asked to treat the phantom once as they would treat a normal patient. The phantom was loaded with dosimeters and irradiated. No major dosimetric problems related to absorbed dose calibration or calculation in the phantom were found.

In 1993, Van Dam and colleagues [5] reported results of mechanical checks of megavoltage units and simulators that were included in the on-site physics programme of the EORTC from 1987. Measurements were obtained in 16 different centres with regard to 23 accelerators, 14 cobalt units and 14 simulators. In general, the deviations observed for accelerators and simulators were smaller than for cobalt units, possibly related to the advanced age (up to 20 years) of some of the latter kind of units. Hansson and colleagues [6] reported the mailed thermo luminance dosimeter (TLD) dosimetry programme for machine output check and clinical application which was started in 1986. The therapy machine output checks have revealed a few large deviations $(>7 \%)$ between 
EORTC-measured and institute-stated dose. Special measurements were done for the EORTC trial $22881 / 10882$. It was reported that $80 \%$ of the measured doses were within $5 \%$ from the doses computed in the institute. Horiot and colleagues [7] in 1993 reported on the minimum requirements for $\mathrm{QA}$ in radiotherapy. Several steps of a $\mathrm{QA}$ structure were mentioned. First-line radiation physics and radiotherapy parameters were defined such as: beam calibration; mechanical checks of equipment; dose specifications to prescribe and report radiotherapy; treatment planning system and compliance to a programme of measurements and checks in each radiotherapy department. Methodology of QA in radiation physics should include site visits and mailed TLD. Guidelines were also given regarding the QA of the clinical trials (e.g. data management, case report forms, dummy-runs, identification and score of deviations) and the development, testing and promotion of transfer of new methodologies of QA. In the framework of the experimental implementation of a European QA network for external radiotherapy, a report was published in 1995 on the use of mailed TL dosimeters to check the beam output and the beam quality of the photon beams [8]. Inter-comparisons of the results with the EORTC own mailed TLD, have shown an agreement better than $2 \%$ for all energies.

In 1996, Bernier and colleagues [9] reported the profile of radiotherapy departments participating in clinical trials of the radiotherapy group of the EORTC and compared it with their earlier report [2]. The general results showed that: large variations in equipment and staff were still observed among participating centres; the number of cancer patients treated per year per radiation oncologist seemed to diminish slightly, especially in those centres experiencing a considerable staff shortage before; the most significant improvement was observed for the number of cases treated per year per member of the radiation physics ream; the radiation technologist's workload shows the opposite trend; the situation for equipment is unchanged in comparison with that observed 6 years before. Bentzen and colleagues [10] in 2000 reported the possible clinical impact of dosimetry QA programmes assessed by radiobiological modelling of data from the TLD dosimetry study of the EORTC. In 2001, Gomola and colleagues [11] checked 330 electron beam set-ups in the reference centres and some local centres of the EC Network Project and in addition the centres participating in the EORTC trial 22922. The standard deviation of the measured dose to stated dose was $3.2 \%$, while in seven beam set-ups deviations greater than $10 \%$ were observed, showing the usefulness of external audits of clinical electron beams. 


\subsection{Radiotherapy-clinical trials}

In the early 1980s, the EORTC Radiotherapy Group observed several major problems related to sub-optimal planning, treatment technique and dose distribution. Subsequently, a consensus meeting in 1987 focusing on QA was held. Several recommendations and guidelines were published for the establishment of a programme of QA in radiotherapy inside the EORTC [12]. It was recommended that this centralised QA programme should include site visits, mailed dosimetric measurements and a review of the radiotherapy treatment records. Criteria for acceptable practices were reported concerning dose delivery, deviation tange of prescribed dose and patient immobilisation, as well as verification of (portal) films. Emphasis was given to the mandatory use of the target volume as defined by the International Commission on Radiation Units and Measurements (ICRU) recommendations. In 1990, the EORTC Radiotherapy Group held a consensus meeting in Tübingen, Germany to review the treatment sequence in the conservative management of early breast cancer (EORTC trial 22811/10882: "assessment of the role of the booster dose in breast-conserving therapy") from the viewpoint of QA [13]. The reports of this meeting considered the most relevant criteria for evaluating pathology, surgery and radiotherapy techniques, not only for selected institutions participating in prospective randomised trials, but also aiming at developing QA procedures for use anywhere for routine standard treatment.

A consensus on a QA programme in the treatment of early breast cancer was reached in a multidisciplinary EORTC and European Society of Mastology (EUSOMA) meeting of surgeons, pathologists, radiation oncologists, physicists and radiation technologists [14]. Specific guidelines were established for treatment preparation, careful location and excision with marking of the primary tumour, target volumes for irradiation of the wholle breast and boost area. Radiation close prescription rules, specification and checking procedures were provided, together with guidelines to achieve a homogeneous dose within the target volume. At a QA meeting of the Radiotherapy Group in January 1993 [1.5], three goals were defined: designation of minimum requirements, evaluation of dose-volume effect in normal tissues and predictive tests for a better characterisation of individual radiosensitivity. The programme of QA of the EORTC Radiotherapy Group and a historical review was reported in 1993 by Horiot and colleagues [16]. In this publication, special attention was also given to the definitions of acceptable variation, major and minor deviations detected in clinical trials. One of the main aspects of the QA programme is the establishment of clear and representative end-points in terms of radiation-induced morbidity. In 1995, the EORTC Late Effects Working Group, reported together with the US Radiation Therapy Oncology Group (RTOG) the 
SOMA/LENT scale for the scoring of the late effects of radiation-induced toxicity [17].

The concept of a Master Protocol for phase III studies was realised, in order to make the work of future study co-ordinators easier and to improve the homogeneity in the protocol descriptions of a clinical trial [18]. The Master Protocol defines and explains, in a logical order, the different steps that must be taken in the design of a randomised trial, from the rationale to the references. Particular attention is paid to eligibility criteria, volumes of interest defined according to the recommendations of ICRU Report 50 (gross tumour volume, clinical target volume, planning target volume and organs at risk), simulation procedure, treatment technique, normal tissue-sparing, dose computation, equipment and dose specification. Last, but not least, the different QA procedures are defined (site visits, dummy run procedure, in vivo dosimetry, individual case review) to allow working plans to be made in advance.

The first results of a dummy-run procedure for quality checking in the EORTC trial $22881 / 10882$ were published in 1991 by van Tienhoven and colleagues [19]. Three transverse sections of a patient were sent to 16 participating institutions with a request to make a three-plane treatment plan, according to the protocol prescriptions. The evaluation revealed a large variation in the treatment techniques used, especially concerning the use of wedge filters. Two institutions did not apply wedges, whereas wedge angles in the other institutions varied between 6 and $46^{\circ}$. An examination of the dose-distributions showed that the dose was specified at a point according to the protocol guidelines in 11 institutions, to the 90,95 or $100 \%$ isodose curve in four institutions while in one institution the dose was normalised to a point in the chest wall. Twelve institutions applied lung density corrections in their treatment planning, while nine reported problems with their planning system in off-axis dose distribution calculation and/or the simulation of collimator rotation. Recalculation of the dose at the isocentre showed agreement within $2 \%$ compared with the stated dose. The dose reported in the tumour excision area varied berween 93 and 100\%. In 1997, van Tienhoven and colleagues [20] overviewed the QA in the same EORTC trial (22881/10882) with a progranme consisting of a dummy run procedure, an individual case review procedure, in vivo dosimetry studies and phantom dosimetry studies. Three types of systematic protocol deviations were detecred and defined: deviations due to ambiguities in the protocol prescriptions; deviations not known to the institution, such as mistakes in implementation of treatment planning algorithms resulting in a systematic overdosage or underdosage and inability of an institution to cope with (precise) protocol prescriptions for technical or logistic reasons.

The results of a dummy run involving nine centres participating in a study comparing 
radiotherapy alone with radiotherapy plus hormone therapy in patients with high metastatic risk prostate cancer (EORTC protocol 22863) were reported in 1995 by Dusserre and colleagues [21]. In all centres but one, patients are treated in the same way. The authors reported also that protocol compliance could be improved by a better assessment of the target volume, by using individualised shields and by using computerised tomography (CT) scan images for treatment planning determining beam position on a large number of slices.

In 1998, Valley and colleagues [22] reported results from a dummy run that was organised to test the compliance of participating centres with the guidelines of EORTC protocol 22931, which compared radiotherapy alone with concomitant radiochemotherapy in the postoperative setting for patients presenting with locally advanced head and neck carcinomas. The results showed wide intercentre variations in PTV extensions raising the question of the reproducibility when pooling patients in multicentric trials and a large variability in the field arrangements which was left to the discretion of the investigators. Only three out of 10 of the institutions followed the ICRU 50 recommendations for dose reporting and, moreover, protocol requirements were not met for dose distribution homogeneity in any centre. In 1.999, an EORTC Scientific Strategy Meeting reported the exceptional and pioneering role of the EORTC in the field of QA in cancer research for radiotherapy, surgery, medical oncology and data management [23].

In 2001, Poortmans and colleagues [24] published a report on the dummy run of the EORTC phase III randomised trial 22922/10925 investigating the role of adjuwant internal mammary and medial supraclavicular (IM-MS) irradiation in stage I-III breast cancer. The evaluation of 32 centres showed a number of more or less important deviations in the treatment set-up and prescription. A deviation of the measured dose in the IM-MS region from the prescribed dose was assessed in a large number of evaluated treatment plans. Due to this sub-optimal dose distribution, a real survival benefit of $5 \%$ would be measured as only a 3.8\% benefit. Moreover, Lievens and colleagues [25] by performing an analysis based on the dummy run data of the same trial, showed that if the recommendations of the QA Committee are applied, both standard and alternative IM-MS irradiation techniques produce acceptable dose distributions.

Recently, Julien and colleagues [26] reviewed the medical files of 824 out of 1010 randomised patients ( $82 \%$ ) of $10853 \mathrm{EOR} \Gamma \mathrm{C}$ trial investigating the role of radiotherapy in breast-conserving treatment for ductal carcinoma in situ of the breast. Large variations occurred, particularly in the surgical procedures and histopathological work-up, emphasising the need for establishing uniform guidelines for diagnostic and therapeutic 
procedures.

In 2001, within the framework of the Emmanuel Van der Schueren Fellowship, the EORTC Radiotherapy Group will complete QA in three protocols:

- EORTC trial 22961: Long- term adjuvant hormonal treatment with luteinising hormone-releasing hormone (LHRH) analogue versus no further treatment in locally advanced prostate carcinoma treated by external irradiation and a 6-months combined androgen blockade-a phase III study.

- EORTC trial 22921: Four arm phase III clinical trial for T3-T4 resectable rectal cancel: comparing preoperative pelvic irradiation with preoperative irradiation combined with 5-fluorouracil and leucovorin with or without postoperative adjuvant chemotherapy.

- EORTC trial 22922/10925: Phase III randomised trial investigating the role of adjuvant IM-MS irradiation in stage I-III breast cancers.

The group continues its efforts in the area of QA with the aim of increasing and consolidating the quality of clinical research and routine daily practice in radiotherapy.

\section{Discussion}

In onder to put into perspective both the orientations that the promoters wanted to give to a project of QA in Radiotherapy and the strategies followed during the development and implementation of the relevant control procedures, it is necessary to revisit the expectations that the EORTC Radiotherapy Group had claimed, when it embarked on this long-term programme. Firstly, it was felt that this QA programme should be a valuable tool to both harmonise treatments and provide clinicians and decision-makers with information on optimal workloads for equipment and human resources. Secondly, this project should have helped EORTC investigators to develop harmonised QA procedures in the therapeutic management of widespread diseases to improve, on a largescale, local control and survival, and reduce the severity of treatment complications. Thus, one must ask how to interpret the clinical relevance of the achievements mentioned above. The analysis of the clinical impact of a QA Programme has to be stratified according to both radiation physics and clinical parameters.

\subsection{Physical parameters}

In 1993, Dutreix and colleagues [27] reported on the preliminary results of a QA network for radiotherapy centres in Europe, showing that the large majority of the beams 
(23/25) with deviations $>3 \%$ belonged to centres that did not participate in external audits in the previous 5 years. Moreover; Hansson and colleagues [6], reporting measurements from mailed dosimetry during a period of 6 years (1987/1992), showed an acceptable level of deviation $( \pm 4 \%)$ between the EORTC measured dose and the institute-stated dose value. However, the most important results coming from this study concerned the positive impact of re-checking using mailed TLDs. The mean rario subsequently approached unity, and the standard deviation decreased, as the number of consecutive mailings increased. This improvement can be explained by corrections that had been made by the participating institutions after a timely feedback regarding their dosimetric status. Correlating information from the TL measurements and the on-site visits led to further improvements in the beam deviations. This report is an exceptional example of the potential role of mailed TLD and local QA audits. Mailed TLD checks should be an integral part of a continuously ongoing QA activity in radiotherapy. In 1994, the European Society for Therapeutic Radiology and Oncology (ESTRO) published an advisory report to the European Commission within the framework of its 'Europe against Cancer' Programme [28]. The main item of this report was the documentation of guidelines for QA in radiotherapy. Moreover, ESTRO offers to its members the possibility to participate in a programme called EQUAL, an acronym for ESTRO QA in radiotherapy [29]. The aim of this network, is to stimulate more permanent action at the level of the EU member states, typically for those smaller centres which up to now did not have the opportunity to participate in any national or international QA programme. In 2000, Ferreira and colleagues [29] published the first report of measurements regarding the EQUAL project in 102 centres. Approximately $3 \%$ of the outputs in reference conditions show deviations outside tolerance level ( $>5 \%$ ). The ESTRO-EQUAL report together with a study by Gomola and colleagues emphasised the feasibility and the potential role of external audits with mailed TLDs in detecting deviations in electron or photon beams $[11,29]$. It seems that nowadays the instruments for measuring the quality of radiotherapy treatment and performing QA activities are available, easy-to-use and reliable. The final implementation of the QA procedure remains the only problem.

Importantly enough, the EORTC Radiotherapy Group was the first to demonstrate, by means of a Quality Assurance Programme, that small deviations in beam output may lead to clinically important variations in outcome. Bentzen and colleagues [10] by a rigorous evaluation showed that in $10 \%$ of the beams with the most pronounced under-dosage, the loss in tumour control probability could be up to 7-8\%. Likewise, in the $10 \%$ of the beams with the most pronounced overdosage, the calculated increase in the probability 
of mild/moderate morbidity would be up to 19-22\%. Moreover, for severe morbidity, the same beams raised the estimated probability of severe complications from 5 to 9-10\%. The decrease in the probability of an uncomplicated cure was estimated at $1 \%$ both for high and low-energy beams; sequential mailings considerably improved the uniformity of clinical outcome. The clinical impact of beam dose deviations from the stated dose is certain.

The infrastructure of a radiotherapy department can also have an impact on QA outcome, and this should not be underestimated [9]. The organisation of a department should also be subject to a QA system. Leer and colleagues [30], described how the socalled ISO 9001 quality standards can be applied to create a QA system in a hospital department.

\subsection{Radiotherapy clinical trials}

QA procedures allow a good estimation of patient-to-patient and inter-institutional variations, and early detection of (potential) systematic protocol deviations of 3 types [20]: deviations due to ambiguities in the protocol prescriptions; deviations not known to the institution, such as mistakes in the implementation of treatment planning algorithms resulting in a systematic over-dosage or under-dosage; and the inability of an institution to cope with (precise) protocol prescriptions for technical or logistic reasons. The first two types of deviations may be corrected or avoided by direct discussions with the study co-ordinator, specific recommendations for the dose in the ICRU point and in vivo dosimetry. With respect to the third type of deviation, it is up to the trial coordinator to accept or refuse a centre's participation, depending upon the relative importance of the particular deviation(s) for the trial endpoints. To be effective, such a QA programme must be implemented as early as possible in the course of a clinical trial $[18,20]$.

This historical review showed, in general, many problems concerning protocol inconsistencies and routine clinical practice. Potential systematic protocol deviations possibly leading to false-negative/-positive results are often detected $[10,19,20,22,24,25]$. Large or small intercentre variations in planning target volume (PTV) extensions are also recorded. This fact often raises the question of reproducibility when pooling patients in multicentric trials [22]. Unfortunately, not all institutions followed the ICRU 50 recommendations for dose reporting [19,20,22,24,25]. Protocol requirements are often not met for dose homogeneity in participating centres [10,19,20,22,24,25]. Moreover, the possible influence of deviations in treatment delivery on the end-results of studies was calculated and this fact emphasised the immense value of QA in clinical trials $[10,24,25]$. 
This retrospective analysis clearly shows that, throughout the last decade, the Cooperative Group for Radiotherapy has been able to extend its basic quality assurance of equipment and dosimetry into prospective investigations consisting of pilot studies for systematic checkings of individual treatment and treatment reliability, resulting in a large body of data on treatment precision level, systematic deviations and individual errors. The tackling of systematic and random errors has been extremely successful since the set-up of QA procedures such as the dummy-runs and individual case reviews enabled the identification of the major sources of ambiguities, as well as all causes of poor compliance to the protocols, resulting in the release of helpful recommendations for all participating centres.

QA programmes are not only well accepted by all participants, but also felt by everyone to be mandatory for the validity of cooperative work between several centres. This project has and continues to provoke lively and very constructive discussions within the group, especially during the last 5 years where individual contacts among investigators and local teams have been promoted. From the experience gained by the Radiotherapy Group, the main axes of research are now centered around the following issues:

- Cost-benefit analysis of the dummy-runs, case report forms and individual case review procedures, in specific randomised trials of the Radiotherapy Group of the EORTC.

- Update of information on required radiotherapy infrastructure in EORTC institutions based on mailed questionnaires, in collaboration with ESTRO and possibly in the frame of a concerted action supported by the European Commission.

- Through the use of telematic services, the EORTC Radiotherapy group will also investigate the feasibility of teleconferencing QA audits by physicists and medical specialists for new radiotherapy techniques such as three-dimensional-conformal radiotherapy (3D-CRT) and intensity-modulated radiation therapy (IMRT) planning.

- To identify all prerequisites related to an improved efficacy of 'integrated' QA programmes, which consider the accuracy of radiotherapy in the framework of mulridisciplinary treatments and require a prospective implementation of various Quality Systems. 


\section{Conclusions}

A QA system should include an interactive feedback procedure in all steps of a clinical protocol and/or of the radiation treatment (prescribed dose, treatment planning, patientpositioning, etc.); corrections should be made immediately after an audit. The main aspect of QA is not only to diagnose an error or inconsistency, but also to give advice, aiming to improve protocol compliance and to ensure that corrections are made as soon as possible. Thus, a QA structure should be friendly, informal and confidential, without aggressive censuring of an investigator/institution, but aiming at improving the quality of their routine dinical practice. Results of a QA procedure should always be published anonymously.

From the experience gained in the EORTC Radiotherapy Group, it is clear that all opportunities to transfer the knowledge of QA have to be identified and enforced on a large-scale between institutions inwolved in clinical research and lay community hospitals. This should be done by exchanging experience on QA procedures applying to radiation physics parameters, transferring these into daily practice pilot Quality Systems that are able to monitor the effects of measures taken in the context of multidisciplinary treatments and expanding information networks on the generic quality standards developed for new radiotherapy techniques.

\section{References}

1. World Health Organization (WHO), Qualicy Assurance in Radiorherapy. Geneva, WHO, 1988.

2. Horior JC, Johansson KA, Gonzalez $D G$, van der Schueren $E$, van den Bogaer W, Notrer $G$. Quality assurrance control in the EORTC cooperative group of radionherapy. 1. Assessment of radiotherapy staff and equipment. European Organization for Research and Treatment of Cancer. Radiother Oncol 1986, 6, 275-284.

3. Johansson KA, Horior JC, Van Dam I, Lepinoy D, Sentenac I, Sernbo G. Quality assurance control in the EORTC cooperative group of adiontherapy. 2. Dosimetric intercomparison. Radiother Oncol 1986, 7, 269-279.

4. Johansson KA. Horiot JC. van der Schteren E. Quality assurance control in the EORTC cooperatiwe group of radiotherapy. 3. Lntercompatison in an anatomical phantom. Radiorher Onool 1987, 9, 289-298.

5. Van Dam J, Johansson KA, Bridier $A$, Sembo $G$, Hansson U. EORTC radioherapy group quality assurance: medranical checks and beam alignments of megavoltage equipment. Radiother Oncol 1993, 29, 91-96.

6. Hansson U, Johansson KA, Horiot JC, Bernier J. Mailed TL dosimetry programme for machine outpur check and dinical application in the EORTC laddiotherapy group. Radiother Oncol 1993, 29, 85-90.

7. Horiot JC, Bernier J. Johansson KA, van der Schueren E, Bartelink H. Minimum requirements for quality assurance in radiotherapy. Radiother Oncol 1993,29, 103-104.

8. Derteumaux S, Chawadra I, Bridier A, Rosserti V, Dutreix A. European quality assurance nerwork for radiorherapy: dose measurement procedure. Phys Med Bioll 1995, 40, $1191-1208$.

9. Bernier J, Horiot JC, Bartelink H, et al Profile of radiotherapy departments contributing to the Cooperative Group of Radiotherapy of the European Organizarion for Research and Trearment of Cancer. Int I Radiar Oncol Biol Phys 1996, 34, 953-960.

10. Bentzen SM, Bernier J, Davis JB, et al. Clinical impact of dosimetry qualiry assurance programmes assessed by radiobiological modeling of data from the thermoluminescent dosimerry study of the European Organization for Research and Treatment of Cancer. Eur I Cancer 2000, 36, 615-620. 
11. Gomola I, Van Dam J, Isern-Verdum I, er al. Exrernal audirs of electron beams using mailed TLD dosimerry. preliminary results. Radiother Oncol $2001,58,163-168$.

12. Johansson KA, Hanson WF, Horior JC. Workshop of the EORTC Radiotherspy Group on qualing assurance in cooperative trials of radiorherapy: a recommendation for BORTC Cooperative Groups. Radiother Oncol 1988. $11,201-203$.

13. Horior JC. Rationale for a guality assurance programme in clinical crials of conservative management of breast carcinoma. European Organization for the Research and Treatment of Cancer. Radiorher Oncol 1991, 22, 222-225.

14. Bartelink $H$, Garavaglia $G$, Johansson $K A$ er al. Quality assurance in conserwative treatment of exirly breast carcer. Report on a consensus meeting of the EORTC Radiotherapy and Breast Cancer Cooperative Groups and the EUSOMA (European Sociery of Mastology). Radiother Oncol 1991, 22, 323-326.

15. Bartelink H, Bernier J, Kurtz J, Garavaglia G. Radiother Oncol 1993, 29, xi-xïi.

16. Horior JC, van der Schueren E, Johansson KA, Bernier J, Bartelink H. The programme of qualiry assurance of the EORTC radiotherapy group. A historical review. Radiorher Oncol 1993, 29, 81-84.

17. Payy J], Denekamp ], Letschert J, er al. EORTC Lare Effects Working Group. Late effecrs toxicity scoring: the SOMA scale. Radiother Oncol 1995, 35, 11-15.

18. Bolla $M$, Bartelink $H$, Garavaglia $G$, et al. EORTC guidelines for writing protocols for clinical urials of radiotherapy. Radiother Oncol 1995, 36, 1-8.

19. wan Tienhoven $G$, wan Bree NA, Mijnheer BJ, Bartelink $H$. Quality assurance of the EORTC trial 22881/10882:"assessment of the role of the booster dose in breast conserving therapy": the dummy run. EORTC Radiorherapy Cooperative Group. Radiother Oncol 1991, 22, 290-298.

20. van Tienhoven $G$, Mijnheer BJ, Bartelink $H$, Gonzalez DG. Quality assurance of the EORTC Trial 22881/10882:boost versus no boose in breast conserving therapy. An overwiew. Srrahlenther Onkol 1997, 173, $201-207$.

21. Dusserre A, Garavaglia G, Giraud JY, Bolla M. Quality assurance of the EORTC radiotherapy trial 22863 for prostatic cancer: the dummy run. Radiorher Oncol 1995, 36, 229-234.

22. Valley JF Bernier J, Tercier PA, et al. Quality assurance of the EORTC radiortherapy trial 22931 for head and neck carcinomas: the dummy run. Radiother Oncol 1998, 47, 37-44.

23. Meunier F, Horiot JC. EORTC scientific strategy meeting 25-26 March 1999. Eur J Cancer 1999, 35 , $1423-1430$.

24. Poortmans PM, Venselaar JL, Struikmans H, et al. The potential impact of treatment variations on the results of radiotherapy of the internal mammary lymph node chain: a quality-assurance report on the dummy run of EORTC Phase III randomised trial 22922/10925 in Stage I-III breast cances: Int J Radian Oncol Biol Phys 2001, $49,1399-1408$.

25. Lievens Y, Poortmans P, Van den Bogaert W. A glance on quality assurance in EORTC study 22922 evaluating techniques for internal mammary and medial supraclawicular lymph node chain iradiation in breast cancer. Radiorher Oncol 2001, 60, 257-265.

26. Bijker N, Rurgers EJ, Peterse $\int L$, et al. Variations in diagnostic and therapeutic procedures in a mulricentre, randomized dinical trial (EORTC 10853) investigating breast-conserving treatrnent for DCIS. Rur J Surg Oncol $2001,27,135-140$.

27. Durreix $A$, van der Schueren E, Derreumaux S, Chavaudra J. Preliminary resulte of a qualiry assurance network for radiotherapy centres in Europe. Radiother Oncol 1993, 29, 97-101.

28. Thwates D, Sealliet P, Leer JW, Overgaard ]. QA in radiotherapy; ESTRO report 1994. Radiother Oncol 1995. $35,61-73$.

29. Ferreira IH, Durreix A, Bridier A, Chavaudra J, Svensson H. The ESTRO-QUALiry assurance network (EQUAL). Radiother Oncol 2000, 55, 273-284.

30. Leer JW, Corwer R, Kraus JJ, v d Togt JC, Buruma OJ. A quality assurance system based on ISO standards: experience in a radiotherapy deparment. Radiother Oncol 1995, 35, 75-81. 



\section{CHAPTER 3}

\section{Recurrence rates after treatment of breast cancer with standard radiotherapy with or without additional irradiation.}

Harry Bartelink, Jean-Claude Horiot, Philip Poormans, Henk Struikmans, Walter wan den Bogaert, Isabelle Barillot, Alain Fourquet, Jacques Borger, Jos Jager. Willem Hoogenrad, Lawrence Collette, Marianne Pieran, for the European Organization for Research and Treatment of Cancer Radiotherapy and Breast Cancer Groups.

N EnglJ Med 345: 1378-1387, 2001. 


\section{Abstract}

Background: Radiotherapy prevents local recurrence of breast cancer after breastconserving surgery. We evaluated the effect of a supplementary dose of radiation to the tumor bed on the rates of local recurrence among patients who received radiotherapy after breast-conserving surgery for early breast cancer.

Methods: After lumpectomy and axillary dissection, patients with stage I or II breast cancer received $50 \mathrm{~Gy}$ of radiation to the whole breast in 2-Gy fractions over a five-week period. Patients with a microscopically complete excision were randomly assigned to receive either no further local treatment (2657 patients) or an additional localized dose of 16 Gy, usually given in eight fractions by means of an external electron beam (2661 patients).

Results: During a median follow-up period of 5.1 years, local recurrences were observed in 182 of the 2657 patients in the standard-treatment group and 109 of the 2661 patients in the additional-radiation group. The five-year actuarial rates of local recurrence were 7.3 percent (95 percent confidence interval, 6.8 to 7.6 percent) and 4.3 percent ( 95 percent confidence interval, 3.8 to 4.7 percent), respectively ( $\mathrm{P}<0.001$ ), yielding a hazard ratio for local recurrence of 0.59 (99 percent confidence interval, 0.43 to 0.81 ) associated with an additional dose. Patients 40 years old or younger benefited most; at five years, their rate of local recurrence was 19.5 percent with standard treatment and 10.2 percent with additional radiation (hazard ratio, 0.46 [99 percent confidence interval, 0.23 to 0.89 ]; $P=0.002$ ). At five years in the age groups 41 to 50 years old, 51 to 60 years old, and above 60 years additional radiation reduced local recurrence rates from 9.5 percent (99 percent confidence interval, 8.2 to 10.7 percent) to 5.8 percent (99 percent confidence interval, 4.8 to 6.8 percent), from 4.2 percent $(99$ percent confidence interval, 3.5 to 4.9 percent) to 3.4 percent ( 99 percent confidence interval, 2.7 to 4.1 percent), and from 4.0 percent (99 percent confidence interval, 3.2 to 4.7 percent) to 2.5 percent $(99$ percent confidence interval, 1.9 to 3.2 percent), respectively. No differences were found in rates of metastasis or overall survival (which were 87 and 91 percent, respectively?.

Conclusions: In patients with early breast cancer who undergo breast-conserving surgery and receive $50 \mathrm{~Gy}$ of radiation to the whole breast, an additional dose of $16 \mathrm{~Gy}$ of radiation to the tumor bed reduces the risk of local recurrence, especially in patients younger than 50 years of age. 


\section{Introduction}

Several randomized clinical trials [1-5] and a meta-analysis [6] have shown similar survival rates after breast-conserving therapy and after mastectomy in patients with early breast cancer. The B-06 trial of the National Surgical Adjuvant Breast and Bowel Project and other trials demonstrated that after microscopically complete excision, irradiation of the whole breast with a dose of 50 Gy reduced the rate of recurrence in the ipsilateral breast from 35 percent to 10 percent. [4,7-10] However, little is known about the effect of irradiation of the breast at doses higher than $50 \mathrm{~Gy}$.

In the absence of data from randomized trials, there is no uniformity in the dose of radiation and the schedules of fractionation that are used after breast-conserving surgery. To investigate the effect of an additional dose of radiation (a boost) aimed at the tumor bed after the administration of $50 \mathrm{~Gy}$ to the whole breast, the European Organization for Research and Treatment of Cancer (EORTC) designed the present trial. We report the first analysis of outcomes in patients with completely excised primary tumors who were randomly assigned to receive the standard therapy - 50 Gy of radiation to the whole breast - or standard therapy plus an additional dose of radiation of 16 Gy directed at the tumor bed.

\section{Methods}

\subsection{Siudy Design}

Between 1989 and 1996, we enrolled 5569 patients with stage I or II breast cancer who had undergone macroscopically complete surgical removal of the rumor and axillary dissection. The 5318 patients in whom the tumor had been completely excised on microscopical examination (as judged on the basis of the parhology report) were randomly assigned to undergo 50-Gy irradiation of the whole breast with or without an additional dose of $16 \mathrm{~Gy}$ to the tumor bed; 2661 patients were assigned to the additionalradiation group and 2657 to the standard-treatment group. Patients with a microscopically incomplete excision were assigned to receive booster doses of 10 or 26 Gy; these 251 patients were excluded from the analyses reported here. At randomization, patients were stratified according to age, menopausal status, the presence or absence of an intraductal component in and around the invasive tumor, clinical tumor size, clinical nodal status, and center. The sample size was calculated so as to give the study a 90 percent probability of detecting a 5 percent improvement in 10-year survival. An independent data monitoring committee was convened before 10 years had elapsed. The committee advised that we publish the data on local control of the cancer after a median 
of five years of follow-up, since a statistically significant effect of the higher dose of radiation on the rate of local recurrence was clearly demonstrated and since it was concluded that these results were unlikely to change with additional follow-up.

\subsection{Eligibility}

Patients with breast cancer of clinical stage $\mathrm{T1}-2, \mathrm{~N} 0-1, \mathrm{M} 0$ were eligible for the trial. [11] Patients older than 70 years of age, or those with pure carcinoma in situ, multiple tumor foci in more than one quadrant, a history of other cancers, an Eastern Cooperative Oncology Group (ECOG) performance score higher than 2, residual microcalcifications on mammography, or gross residual disease in the breast after lumpectomy (unless reexcision had been performed) were ineligible. Oral informed consent was obtained according to the guidelines of the EORTC and the local and national rules of the participating centers. Patients came from 31 centers in nine countries. There were major violations of eligibility criteria in the cases of 26 patients - residual microcalcifications on postoperative mammography ( 6 patients), previous history of a malignant tumor ( 5 patients), pure intraductal carcinoma in situ ( 3 patients), stage T3 tumor ( 2 patients), clinically fixed axillary nodes ( 2 patients), and various other violations ( 8 patients). In addition, 107 patients were older than 70 years, and for 343 patients the delay between surgery and the start of radiotherapy was longer than that allowed by the protocol. The latter two groups were considered to have minor deviations from the protocol. All patients, whether eligible or ineligible, were included in the analysis.

\subsection{Treatment}

The protocol called for patients to undergo surgical excision of the primary tumor, with a $1-\mathrm{cm}$ margin of macroscopically normal tissue (lumpectomy), and an axillary dissection. Any removal of additional breast tissue after the excision of the primary tumor was termed a reexcision, whether it was performed during the same session or later. Postoperative mammography was required if suspicious microcalcifications were seen before lumpectomy. The surfaces of specimens were routinely marked with India ink in the participating centers. Any invasive carcinoma that was found on microscopical examination to have crossed the inked margin was defined as incompletely excised. Patients with a microscopically incomplete excision underwent randomization separately to an additional dose of radiation of either $10 \mathrm{~Gy}$ or $26 \mathrm{~Gy}$. Patients with axillary lymphnode involvement received adjuvant systemic therapy: premenopausal patients received chemotherapy, and postmenopausal patients received tamoxifen. Patients not given adjuvant chemotherapy began radiotherapy within nine weeks after lumpectomy. For patients who received adjuvant chemotherapy, a delay of up to six months before 
irradiation was allowed. Irradiation of the whole breast was performed with the use of two tangential megavoltage photon beams (high-energy $x$-ray or tele-cobalt). A total dose of 50 Gy over a five-week period, with a dose of $2 \mathrm{~Gy}$ per fraction, was delivered at the intersection of the central axes of the beams. [12] The additional dose of $16 \mathrm{~Gy}$ was given to the center of the area from which the tumor had been excised; it was given in eight equal external-beam fractions with fast electrons or tangential photon fields, or alternatively, by means of an iridium-192 implant with a dose rate of 10 Gy per 24 hours. [12-15]

The target area for the additional dose of radiation was the site of the primary tumor, with a margin of $1.5 \mathrm{~cm}$ around the primary tumor after microscopically complete excision and of $3.0 \mathrm{~cm}$ after incomplete excision or in case of invasive cancer with extensive ductal carcinoma in situ.

\subsection{Quality Assurance}

At the start of the trial, the participating physicians received guidelines, $[13,14]$ a qualityassurance program was implemented to confirm that breast irradiation was carried out in a standard fashion in all centers, [14-17] a team of physicists verified the calibration of the beams in the participating centers, and a program was set up for thermoluminescent dosimetry. If a major deviation between the calculated doses and the delivered doses was detected, a local audit was held to identify the reason. A central pathology review was performed by one pathologist at the Netherlands Cancer Institute.

\subsection{Statistical Analysis}

All analyses were conducted according to the intention-to-treat principle. The randomization was performed centrally at the EORTC Data Center by means of the minimization technique. [18] The time to local recurrence was calculated from the date of randomization. All recurrences in the treated breast, before or after the detection of distant metastases, were taken into account. Data for parients who remained free of local disease were censored as of the date when the last follow-up information was obtained. The primary analysis of time-to-event end points was performed with the use of a twosided log-rank test at the 5 percent level of significance. [19]

Analysis was performed on subgroups defined by the factors that had been used for stratification before randomization: age, menopausal status, presence or absence of ductal carcinoma in situ, clinical tumor size, and nodal status. The probability of event-free survival was estimated with the Kaplan-Meier technique. For the analysis of prognostic factors, we used a Cox proportional-hazards regression model, stratified according to treatment-group assignment. [20] These latter analyses included all 5569 patients. 
Univariate and multivariate analyses were conducted to determine which factors were associated with local control of the cancer. A univariate analysis was conducted first, and then a backward-selection procedure was used to determine the composition of the multivariate models. Initially, all the variables that were significant at the 0.05 level in the univariate analysis were entered in the modell. The least significant variables were then sequentially removed from the model. The last variables to be removed were checked for reentry into the model, in order to validate the final model. For the multivariate analyses, the models obtained with both the 0.05 and 0.01 levels of significance are described.

\section{Results}

\subsection{Characteristics of the Patients, the Tumors, and the Treatments}

Characteristics of the patients were similar in the two groups (Table 1). To achieve a microscopically complete resection, reexcision was required in 24 percent of the patients. In 99 percent of the patients, axillary dissection was performed. The median number of axillary nodes examined was 12 (range, 0 to 49 ), and in 60 percent of the patients more than 10 lymph nodes were examined. A total of 13 percent of the patients had a hematoma or breast infection as a complication. Another 17 percent had a seroma or an infection of the axillary wound. The median interval between lumpectomy and the start of radiotherapy was 39 days (range, 3 to 156) for patients who did not receive postoperative chemotherapy and 52 days (range, 14 to 469) for those who did receive postoperative chemotherapy. The median dose of radiation delivered to the tumor bed was 50 Gy (range, 2 to 73) in the standard-treatment group and $66 \mathrm{~Gy}$ (range, 23 to 79) in the additional-radiation group. Axillary irradiation was given to the 272 patients who did not undergo a complete axillary dissection or to patients with positive lymph nodes in the highest level of the axillary surgical specimen. Irradiation of the internal mammary lymph nodes was performed in 1089 of the patients. In the standard-treatment group, 53 (2 percent) nevertheless received an additional dose, and the treatment was not documented in 24 patients ( 1 percent). In the additional-radiation group, 26 patients ( 1 percent) received no additional dose and for 21 patients ( 0.8 percent) the information was missing. These patients' treatments were considered to be protocol violations; 


\begin{tabular}{|c|c|c|c|c|c|}
\hline CHARABATEATSTE: & $\begin{array}{c}\text { STMKDART- } \\
\text { TAEATHATI } \\
\text { GFOUP } \\
(N=2657)\end{array}$ & 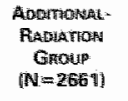 & CHARACTESUSTK: & 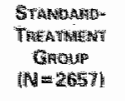 & 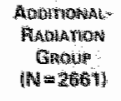 \\
\hline \multicolumn{6}{|c|}{$y e$} \\
\hline \multicolumn{6}{|l|}{ Pariente } \\
\hline A $\$ g C$ & & & & & \\
\hline Nedtatio & 54.9 & 54.6 & & & \\
\hline Rangze & $22.7-83.5$ & $25.6-78.48$ & & & \\
\hline & \multicolumn{2}{|c|}{ no. $\{x\}$} & & \multicolumn{2}{|c|}{ no. } \\
\hline Age gromp & & & Thumor converuned ly & & \\
\hline$-35 y$ & $72(27)$ & $82(3.1)$ & Wistologe rype at carcionomas & & \\
\hline $30-40 y$ & $15(5.5)$ & $139,5,2)$ & Unkin?owit & $(0,3)$ & $(0,3)$ \\
\hline $41-50 y t$ & 66525.00 & $660(251)$ & lankasive dueral & $2155(81,1)$ & $2198(236)$ \\
\hline $51-60$ yr & $943(355)$ & $860(3 \geq 3)$ & 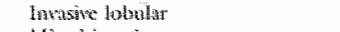 & $228(8.6)$ & $20(0,2)$ \\
\hline 360 ye & $82,30,9)$ & $911(342)$ & Mixed invatsive & $(45)(2,4)$ & BI. $\{,(1)$ \\
\hline Mcynoparusal sentris & & & Tubula & $99(3,7)$ & $7[(2.7)$ \\
\hline Ustenow & $10(04)$ & $8(0.3)$ & Medatllary & $58(2.2)$ & $49(1.8)$ \\
\hline Premanomasal & $999(37.6)$ & $1004\{37.7\}$ & Golloid & $37(1.4)$ & $33(4.2)$ \\
\hline 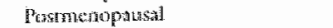 & $1648,62.0 j$ & $6049(62.0)$ & Othes: & $7(0,3)$ & (t) $(1)$ \\
\hline DCOG perfontanace scoren & & & Nis, of nodes extuninded & & \\
\hline Gnknown & $10(04)$ & $9(0,3)$ & Unkionsy & $69(26)$ & $7 \%(2)$, \\
\hline 6 & $2335(879)$ & $2335(877)$ & 0 & $21(0,8)$ & $10(0,6)$ \\
\hline $1-2$ & $312(1), 7\}$ & $317(11.9)$ & $1-5$ & $170(6.4)$ & $170(6,6)$ \\
\hline Tuintior & & & $6-10$ & $83.3(30.6)$ & $820: 3104$ \\
\hline Saze deremined by pulpation & & & $11-15$ & $876(3,0)$ & $914\left\{\begin{array}{l}3 \\
4\end{array}\right\}$ \\
\hline Unknown & $336(12.6)$ & $348(13.1)$ & $>15$ & $708<20.6)$ & $654(24 ; 6,6)$ \\
\hline Not polpable & $509(21.4)$ & $581(21.9)$ & Nortalener of positive markes & & \\
\hline$<\mathrm{cm}$ & $315(11.9)$ & $313(11.8)$ & Unk国omit & $25(0.9)$ & $20(0)$ \\
\hline $1-1.96 \mathrm{mi}$ & $856,32.2 j$ & $820(312)$ & 0 & $2078(75.2)$ & $20 \% 9(78,5)$ \\
\hline $2-3 \mathrm{cmll}$ & $433(16.3)$ & $449(16,7)$ & $1-3$ & $452(1700)$ & $446(169)$ \\
\hline$>3 \mathrm{cmi}$ & $148(5,6)$ & $141(5,3)$ & 34 & $102(3)$ & $10 \geq(3.8)$ \\
\hline 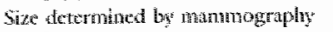 & & & Hotmone-receptor shatust & & \\
\hline$<1 \mathrm{~cm}$ & $5.76(21.7)$ & $525(197)$ & Estrogen & & \\
\hline $1-1.9 \mathrm{~cm}$ & $1027(38.7)$ & $1067(40.1)$ & Nrgaztivit & $525(19.2)$ & $528(19 \mathrm{~s}$ \\
\hline $2-3 \mathrm{~cm}$ & $397(14.97$ & $436(10.4)$ & Posirive & $1391(52.4)$ & $1400(5,0)$ \\
\hline 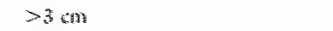 & $110(4.1)$ & $104(3.9)$ & Unknown & $74: 1\langle 279\}$ & $724(27.2)$ \\
\hline Unynowery & 54720.67 & $529(190)$ & Plagesternons? & & \\
\hline Tlitumor stage & & & Negativit & $601\langle 22.6\}$ & 62592355 \\
\hline $\mathrm{T} 1$ & $1379(50.9)$ & $1373(51,6)$ & Positive & $168\langle 44,0\}$ & 11804040 \\
\hline 12 & $1274\{47.9\}$ & $1281\{48.1\}$ & Linkumys! & $888(3.4 .4)$ & $849(3.9)$ \\
\hline 13 & $4(0.2)$ & $7(0.3)$ & Eostroginn and pogresterone & & \\
\hline Notal states & & & Unkmonats & $893(33.6\}$ & 85903210 \\
\hline No & $2409(90.7)$ & $2383\{189,6\}$ & Estrogen pesitive, progersterasto & $10,138.8\}$ & $1042(39.2)$ \\
\hline $\mathrm{N} 1-2$ & $182\{68\}$ & 20097.94 & positive & & \\
\hline$N X^{2}$ & $66(2.5)$ & $69(2.6)$ & Estrogen positike. propesterath & $255(9.6)$ & $208010.0 \%$ \\
\hline Reercision & & & negarive & & \\
\hline Unknowery & $8(0.3)$ & $8(t) 3)$ & 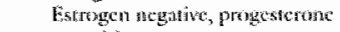 & $133(5,0)$ & $14 \|(5.3)$ \\
\hline s do & $2003,(75,4)$ & $199)(748)$ & powitive & & \\
\hline Yus & $646(243)$ & $662(24,4)$ & 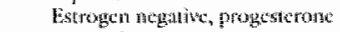 & $3 \times 5(13,0)$ & $358(135)$ \\
\hline 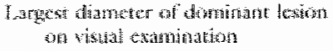 & & & nogarice & & \\
\hline Lnknoway & $49(18)$ & $0 \geq<33$ & & & \\
\hline$<169$ tmon & $683\{25.7$ & $035(230)$ & & & \\
\hline a $0-20$ mut & $402,52.84$ & $1451(545)$ & & & \\
\hline$>20$ in & $523(197)$ & $5113\{19.3\}$ & & & \\
\hline
\end{tabular}

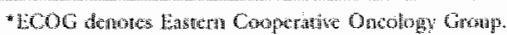

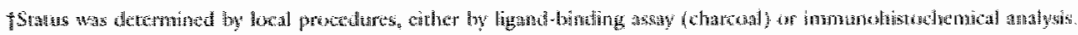

Table 1. Cratacteristics of the Patients and the Tumors

however, they were included in the intention-to-treat analysis. In 90 percent of the patients in this group, external irradiation was used for the additional dose (63 percent with electrons and 27 percent with photons), and in 8 percent an interstitial implant was used; no information was available on the method of delivery of the additional dose for the remaining 1 percent. Details regarding systemic adjuvant therapies are presented in Table 2. 


\begin{tabular}{|c|c|c|c|}
\hline SUEGHOUP & 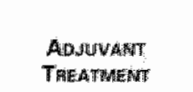 & $\begin{array}{l}\text { STAMLOHAD } \\
\text { TREMTHENIT } \\
\text { GFOUP }\end{array}$ & 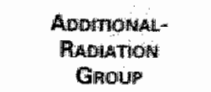 \\
\hline & & \multicolumn{2}{|c|}{ no total wo. with diata in sultogroup (a) } \\
\hline 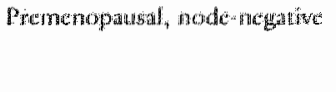 & $\begin{array}{l}\text { Unknowy } \\
\text { None } \\
\text { Chemonherays }\end{array}$ & $\begin{array}{c}5 / 775(0.6) \\
726 / 775(937) \\
44 / 775(5.7)\end{array}$ & $\begin{array}{c}5 / 774(0.6) \\
723 / 774(93) \\
46 / 774(69)\end{array}$ \\
\hline 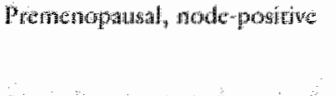 & $\begin{array}{l}\text { Unknown } \\
\text { None } \\
\text { Chementherapy }\end{array}$ & $\begin{array}{c}0 / 221 \\
47 / 221(21.3) \\
174 / 221(78.7)\end{array}$ & $\begin{array}{c}1 / 226(0.4) \\
28 / 226(12.4) \\
197 / 226(87.2)\end{array}$ \\
\hline Pontmenopasisal, hode negratiwe & $\begin{array}{l}\text { Whknowis } \\
\text { None } \\
\text { Chemotherapy } \\
\text { Tamositen } \\
\text { Chemotherapy } \\
\text { and ramoxiten }\end{array}$ & $\begin{array}{c}13 / 1301(10) \\
1072 / 1301(82.4) \\
16 / 1301(1.2) \\
189 / 1301(14.5) \\
11 / 1301(0.8)\end{array}$ & $\begin{array}{c}12 / 1316(0.9) \\
1062 / 1316(80.7) \\
24 / 1316(1.8) \\
212 / 1316(16.1) \\
6 / 1316(0.5)\end{array}$ \\
\hline Postnienophasal, node-positive & $\begin{array}{l}\text { Unknown } \\
\text { None } \\
\text { Chemotherapy } \\
\text { Tamosifen } \\
\text { Chemotherapy } \\
\text { and Emowomen }\end{array}$ & $\begin{array}{r}5 / 333(1.5) \\
22 / 333(6.6) \\
40 / 333(12.0) \\
231 / 333(69.4) \\
35 / 333(10.5)\end{array}$ & $\begin{array}{c}5 / 325(1.5) \\
22 / 325(6.8) \\
29 / 325(8.9) \\
247 / 325(76.0) \\
22 / 325(6.8)\end{array}$ \\
\hline
\end{tabular}

Table 2. Adjuvan Hornonal Treatment or Chemotherapy

\subsection{Follow-up}

The median duration of follow-up was 5.1 years (maximum, 10.2 years), by which point 479 of the 5318 patients with a microscopically complete resection had died and 291 had had local recurrences.

\subsection{Local Control}

Local recurrence was observed in 182 of the 2657 patients assigned to receive only the initial 50 Gy of irradiation of the whole breast and in 109 of the 2661 patients assigned to receive $50 \mathrm{~Gy}$ plus an additional dose of $16 \mathrm{~Gy}$. This incidence corresponds to fiveyear actuarial rates of local recurrence of 7.3 percent $(95$ percent confidence interval, 6.8 to 7.6 percent) and 4.3 percent (95 percent confidence interval, 3.8 to 4.7 percent), respectively $(\mathrm{P}<0.001)$, and a hazard ratio for local recurrence of 0.59 (99 percent confidence interval, 0.43 to 0.81 ) associated with an additional dose (Fig. 1).

Local recurrence was the first event in 5.9 percent of the patients in the standardtreatment group and 3.3 percent of those in the additional-radiation group. In the standard-treatment group, 18 local recurrences were reported after another recurrence (i.e., a distant metastasis, a regional metastasis, or contralateral breast cancer); 9 such events occurred in the additional-radiation group. Overall, 47 percent of the local recurrences occurred in the primary tumor bed, 9 percent in the scar, and 29 percent outside the area of the original tumor, and 27 percent were diffuse throughout the breast. 


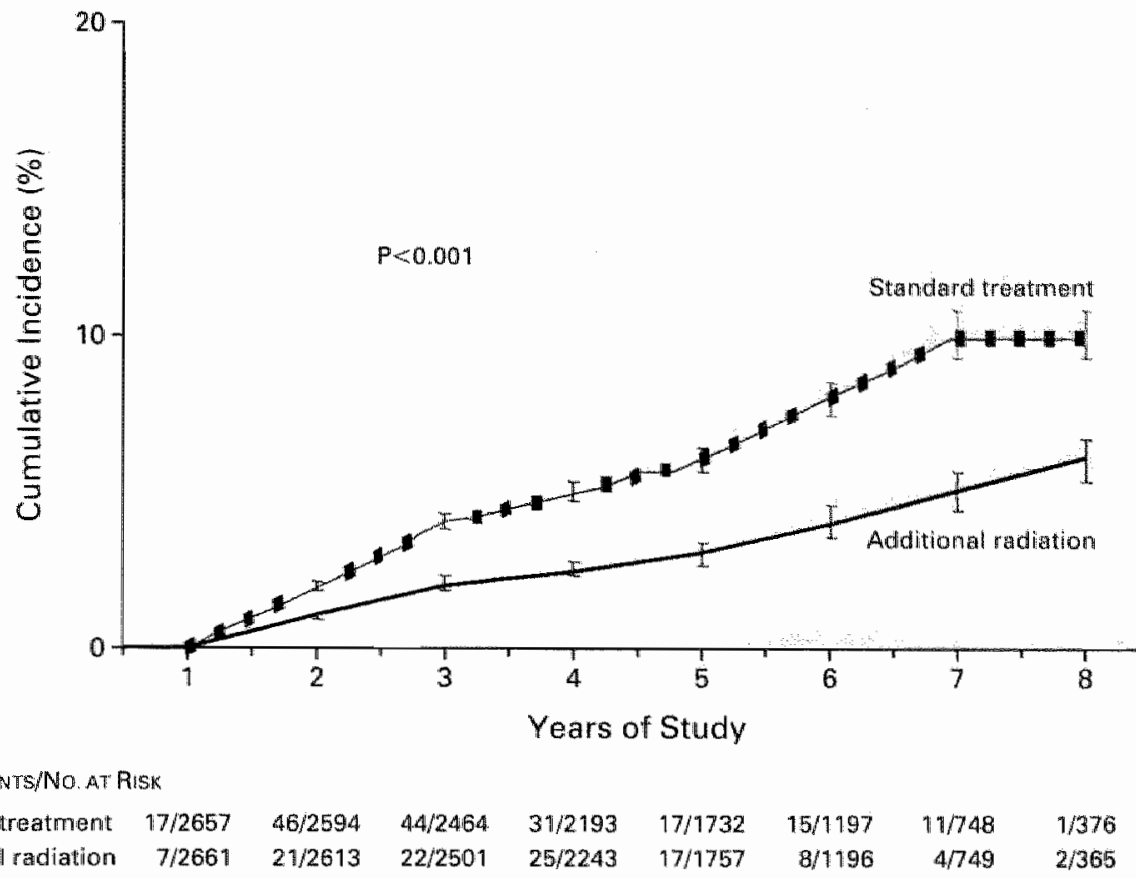

NO. OF EVENTS/NO. AT RisK

$\begin{array}{lrrrrrrrr}\text { Standard treatment } & 17 / 2657 & 46 / 2594 & 44 / 2464 & 31 / 2193 & 17 / 1732 & 15 / 1197 & 1 / 1 / 748 & 1 / 376 \\ \text { Adliditional iadiation } & 7 / 2661 & 21 / 2613 & 22 / 2501 & 25 / 2243 & 17 / 1757 & 8 / 1196 & 4 / 749 & 2 / 365\end{array}$

Figure 1. Cumulative Incidence of Recurrence of Tumor in the Ipsilateml Breast after Whole-Breast Iradiation at 50 G), with or without an Additional Dose to the Tumor Bed.

The P value was calculated by the overall log-rank test for the comparison of the sime to local recurrewce benween the groups. The I bars represent standard deviations.

WAFIASLLE

Age

$\times 40 \%$

$41-50 \mathrm{yT}$

$51-60$ yre

$>60 \mathrm{yr}$

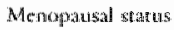

premenopatusis

Postmonopanal

Thtons stage

T2

Nontal shates

No

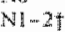

Dunctiol arcimomia in siput

Abscin!

ipesent
STAFHDAFD-TIREATFWIIT GIROUF

No. OF $1 \mathrm{AXCA}$

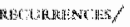

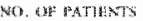
MrThin

46228

611665

43,943

$32 / 821$

$106 / 900$

76,71648

98,1879

$94 / 1274$

169,2409

9,182

$92 / 1641$

$90 / 1015$
ACTHARA

4.

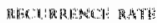

(950) C. H)

parconot
$495(165-225$

$9.5(5.2-10.7)$

$4.2(3,5-2,4)$

$10.3(9.2-11,4)$

$4.6(4.1-5.2\}$

5.9 $\{5.2-6.6\}$

$7.8(7.0-8.7)$

(0.) $(6.4-7.5)$

$5.63 .7 \ldots 7)$

$5.4(4.8-6,0)$

$9.7(82-10) 3)$

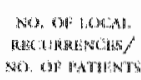

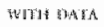

ADdIIONAL-RADMaTION GROUP

Remuction : IN THE

ANMTHAL ODIDS OF

LOCTAL FiELUPAENCE (95\% Cil)

P VALLE:

$$
\begin{aligned}
& \text { MCruaken }
\end{aligned}
$$

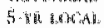

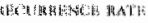

$$
\begin{aligned}
& (95) \mathrm{Cl})
\end{aligned}
$$

porterent

\begin{tabular}{|c|c|c|c|}
\hline $22 / 21$ & $10.2(7.9-12.5)$ & $54(25 \cdots 7)$ & 0,002 \\
\hline $38 / 609$ & $5.8(48-64)$ & $38(8-58)$ & 0.02 \\
\hline $25 / 460$ & $3,1(27,4,1)$ & $26(0-60)$ & 0.07 \\
\hline $24 / 611$ & $2.5(1.9-32)$ & $32(0 \cdots 600)$ & 0.14 \\
\hline $61 / 004$ & $(9.8(5,8,8-7,6)$ & $(x)(1) \ldots 56)$ & 0.001 \\
\hline $45 / 1649$ & $2.8(2.3-32)$ & $41(5-59)$ & 10.604 \\
\hline $50 / 1373$ & $4.0(3.3-4.6\}$ & $42 ! 19-57$ & QOOSL. \\
\hline $58 / 1281$ & $4.5(3,9)-5,2\}$ & $3 x)\{1(3-50\}$ & 0.002 \\
\hline $92 / 2383$ & $4.2\{3,4-\cdots, 6\}$ & $43(27-55\}$ & 0,001 \\
\hline $11 / 209$ & $5.7(3.7 \cdots 7.6)$ & $0(0-56)$ & 0.99 \\
\hline $56 / 10646$ & $3.5(30)-40)$ & sito $\{17-50\}$ & 00102 \\
\hline $53 / 1014$ & $5.6+4.3-6.53$ & $41(17 \cdots 57\}$ & 0,0002 \\
\hline
\end{tabular}

-Cll a denotes somfidence interyal.

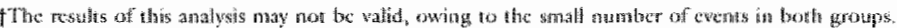

Table 3. Results of Subgroup Analyses of Local Control According to Stratification Factors* 
Analyses of subgroups defined according to the stratification factors (Table 3) found the largest clinical benefit from the higher dose of radiation in patients 40 years old or younger (Fig. 2). The benefit of the additional dose in local control was independent of whether the patients received adjuvant systemic treatment. The hazard ratio was $0.66(99$ percent confidence interval, 0.47 to $0.93 ; \mathrm{P}=0.002$ ) for patients who received no adjuvant treatment, and 0.40 (99 percent confidence interval, 0.20 to $0.81 ; \mathrm{P}=0.001$ ) for patients who received adjuvant treatment.
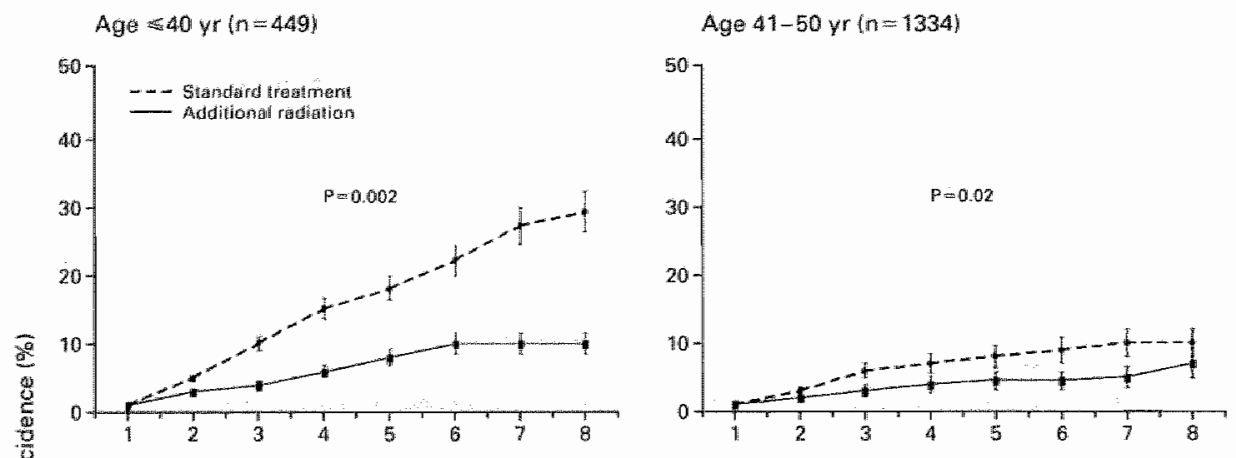

Age $5 \% 11-60$ yr $(n=1803)$

Age $>60$ yr $(n=1732)$
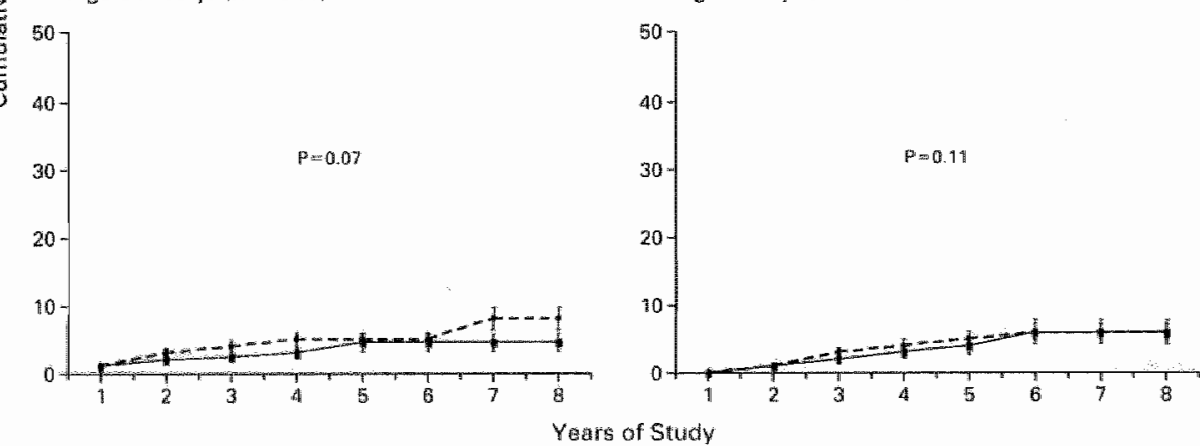

Figure 2. Cumulative Incidence of Recurrence of Tumor in the Ipsilateral Breatst after Whole-Breast Irradiation at $50 \mathrm{~Gy}$, with or without an Additional Dose to the Tumor Bed, According to Age.

Ar fite years, the rate of local recurrence in patients 40 years old or yourger was 10.2 percent in the additionalradiation group, as compared with 19.5 pexcent in the standard-treatment group (hazard ratio, 0.46199 percent confidence interval. 0.23 to 0.891); in patients 41 to 50 years old, the rates were 5.8 percent (99 percent confidence interval, 4.8 to 6.8 percent) and 9.5 percent (99 percent confidence interval, 8.2 to. 10.7 percent), respectively; in patients 51 to 60 years old, the rates were 3.4 percent (99 percent confidence interval, 2.7 to 4.1 percent) and 4.2 percent ( 99 percent confidence interval, 3.5 to 4.9 percent), respectively; and in patients older whan 60 years of age, the rates were 2.5 percent (99 percent confidence interval, 1.9 to 3.2 percent) and 4.0 percent (99 percent confidence interval, 3.2 to 4.7 percent), respectively. P values were calculated by the overall log-rank test for the comparisons of the time to local recurrence between the groups. The I bars represent standard dewations, and $N$ denotes the number of patients in each age group. 


\subsection{Univariate and Multivariate Analysis of Prognostic Factors for Local Control}

In the univariate Cox proportional-hazards regression analysis, patients were stratified according to treatment-group assignment. The following factors had a significant negative effect on local control: young age, premenopausal status, large tumor size (as determined by palpation or as pathologically measured), incomplete first excision of the tumor, absence of estrogen or progesterone receptors, and lack of systemic adjuvant treatment. The variables that reached the 0.05 level of significance in association with age or local control for the overall population were entered in the first step of the multivariate model. Except for the duration of the interval between Jumpectomy and the start of radiotherapy, the total dose to the tumor bed, and the use or nonuse of axillary irradiation, all variables were entered in the first step of the multivariate analysis. The backward-elimination process showed that three variables were significant at $\mathrm{P}<0.05$ : older age ( $\mathrm{P}<0.001$; hazard ratio, 0.59 [95 percent confidence interval, 0.48 to 0.71 ]), the presence of palpable tumors $(\mathrm{P}=0.007$; hazard ratio, 2.14195 percent confidence interval, 1.23 to 3.72$])$, and the presence of a progesterone receptor $(\mathrm{P}=0.004$; hazard ratio, 0.66 [95 percent confidence interval, 0.49 to 0.87 ]), which indicates that the risk of local recurrence was highest in younger patients (Fig. 3), in those in whom the tumor was palpable, and in those who lacked progesterone receptors. At a significance level of 0.01 , the model retained the same factors, but at a significance level of 0.001 it retained only age ( $P<0.001$; hazard ratio, $0.56[99$ percent confidence interval, 0.51 to 0.68$]$ ).

The multivariate analysis of factors associated with local control was repeated with the additional treatment as a covariate as well as the presence or absence of progesterone receptors, the presence or absence of palpable tumor, and age. This analysis demonstrated that all three clinical factors remained significant at the 0.05 level, but onlly age and the use of an additional dose of radiation remained significant at a level of 0.001 . The effect of the additional dose remained significant in this multivariate analysis (hazard ratio, $0.51 ; 95$ percent confidence interval, 0.37 to $0.70 ; \mathrm{P}<0.001)$.

\subsection{Salvage Treatment for Local Recurrences}

Salvage treatment for local recurrences consisted primarily of rotal mastectomy; some patients underwent conservative salvage operations. Mastectomy and lumpectomy were performed in 131 and 24 patients, respectively, among the 182 patients with local recurrence in the standard-treatment group, and 82 and 11 patients, respectively, among the 109 patients with local recurrence in the additional-radiation group; 34 patients received systemic treatment alone or combined with hormonal therapy; and in 9 patients no information was available regarding salvage therapy. 


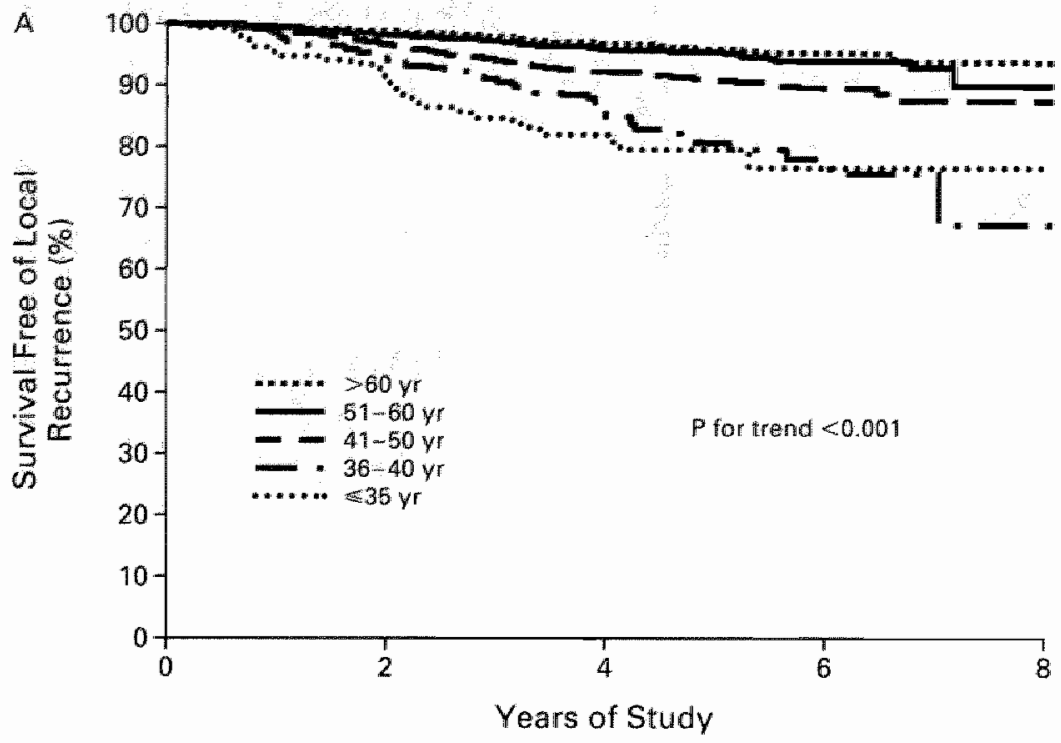

NO AT RISK

$\begin{array}{lrrrrr}>60 \mathrm{yr} & 1607 & 1694 & 1237 & 518 & 85 \\ 51-60 \mathrm{yr} & 1885 & 1768 & 1220 & 525 & 102 \\ 41-50 \mathrm{yr} & 1407 & 1316 & 912 & 407 & 75 \\ 36-40 \mathrm{yr} & 314 & 285 & 200 & 90 & 17 \\ \leqslant 35 \mathrm{yr} & 166 & 137 & 95 & 46 & 9\end{array}$

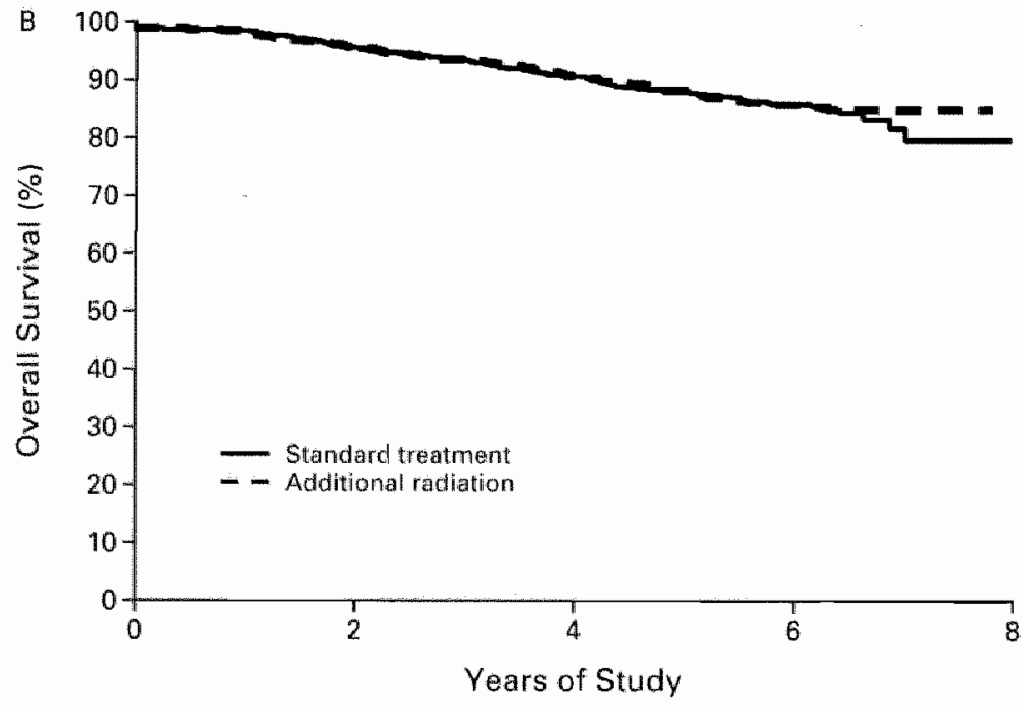

No. AT RISK

Standard treatment 2657

Figure 3. Survinal Free of Local Recurrence According to Age for the Entire Cohort (Panel A) and Overall Survival According to Treatment-Group Assignment (Panel B). There were a total of 58 local recurrences among patients older than 60 years of age. 75 among those 51 to 60 years of age, 109 among those 41 to 50 years of age, 48 among those 36 to 40 years of age, and 28 among those 35 years of age or younger. 


\subsection{Side Effects of the Booster Dose}

The higher dose of radiation was expected to increase the incidence and the degree of fibrosis and cause an unsatisfactory cosmetic result. To date, we have not seen a significant difference in the grades of fibrosis, as scored by physicians on a four-point scale (with 0 indicating the absence of fibrosis and 3 indicaring severe fibrosis) between the patients who received the additional dose and those who did not. The grades of fibrosis in the whole breast for patients in the standard-treatment and additionalradiation groups were as follows: 1288 patients ( 48 percent) and 1339 patients (50 percent), respectively, had no fibrosis; 991 (37 percent) and 908 (34 percent), respectively, had minor fibrosis; $236(9$ percent) and 261 (10 percent), respectively, had moderate fibrosis; 24 ( 1 percent) and 32 ( 1 percent), respectively, had severe fibrosis; and in 118 ( 4 percent) and 121 (5 percent), respectively, the grade of fibrosis was unknown. The cosmetic results were the subject of separate studies and have been published elsewhere. [21,22] Excellent or good cosmetic results were obtained in 86 percent of the patients in the standard-treatment group, as compared with 71 percent of the patients in the additional-radiation group. No other side effects of the additional dose have been observed.

\subsection{Survival and Causes of Death}

Survival free of distant metastases and overall survival were similar in the two treatment groups ( $P=0.93$ and $P=0.63$, respectively), with five-year rates of 87 percent and 91 percent, respectively, in both groups (Fig. 3). No analysis of subgroups defined according to the stratification factors was conducted. The causes of death were also similar in the two treatment groups. Of the 479 deaths, $361-186$ in the standard-treatment group and 175 in the additional-radiation group - were due to breast cancer.

\section{Discussion}

Multiple randomized trials have conclusively demonstrated that breast irradiation markedly reduces the rate of local recurrence after conservation surgery for breast cancer, but these studies did not deal with the dose or fractionation of radiation. The decision to deliver an additional localized dose of irradiation is based on the fact that most local recurrences occur within the vicinity of the primary tumor. For this reason, additional treatment was prescribed in all but one of the randomized trials that established the equivalence of breast-conserving and radical surgical therapy. [1-5] However, the National Surgical Adjuvant Breast and Bowel Project trials limited the dose of radiation to 50 Gy when the excised tumor had microscopically negative margins, a practice that 
has been adopted as standard therapy in published guidelines. [23] Our trial investigated the benefits and risks of adding an additional 16-Gy dose to this standard 50-Gy irradiation of the whole breast in patients with completely excised early breast cancer. We found that an additional dose to the primary tumor area nearly halved the annual odds of local reeurrence (hazard ratio, 0.59). Preliminary data from a smaller trial are similar to ours. [24]

A 41 percent reduction in the rate of local recurrences is substantial in relative terms, but the absolute benefit of additional treatment depends on each patient's risk of recurrence after receiving the initial $50 \mathrm{~Gy}$ of radiation. The only factor we could associate with the extent of the benefit of additional treatment was age. Since the unfavorable influence of young age on the risk of local recurrence and overall survival is well established, [25-29] it was not unexpected that patients 40 years old or younger would derive the greatest absolute benefit from additional treatment; the cumulative five-year rate of local recurrence was 19.5 percent withour an additional dose and 10.2 percent with an additional dose. Our analysis also indicated that breast cancer in younger patients is more responsive to radiation, but there is no clear radiobiologic explanation for this. We do not suggest that additional treatment is ineffective in older patients, but its efficacy does decrease with increasing age, perhaps because in older parients local control after $50 \mathrm{~Gy}$ of irradiation of the whole breast alone is excellent. In our opinion, the absolute benefit of the additional dose justifies its use in patients 50 years old or younger.

The excellent local control found in this study was probably due, at least in part, to the intensive quality assurance program that monitored surgical and radiotherapy procedures and the evaluation of pathology specimens. Unlike many other breast-cancer trials, this study did not involve the use of systemic adjuvant therapy in most patients, although such therapy has been shown to improve local control. We found that systemic adjuvant treatment reduced the rate of local recurrence (hazard ratio, 0.75), although its significance disappeared in the multivariate analysis. The contribution of the additional dose to improved local control was similar regardless of whether patients received systemic adjuvant treatment. Nevertheless, since the patients were not stratified according to the use or nonuse of such treatment, it cannor be considered a substitute for the additional dose, particularly in young patients.

There are indications that the improvement in local control associated with breast irradiation after breast-conserving surgery or mastectomy translates into a survival advantage. $[6,30-32]$ In our trial, however, the five-year survival rate was identical in the two treatment groups. We believe that a follow-up period of at least 10 years would be needed to find a difference in overall survival, and we plan to follow our patients for at 
least 10 years.

Soft-tissue sarcomas are rarely seen after breast irradiation (incidence, less than 0.5 percent). [33] A slight increase in the incidence of contralateral breast cancer was observed in women treated with radiation after mastectomy, probably as a result of the small dose of "scatter" radiation to the other breast. [34] An increase in the incidence of second malignant tumors as a consequence of the higher additional dose has not yet been observed in our trial. However, it is unlikely that the delivery of an additional 16-Gy dose to a limited volume of tissue, with the use of modern irradiation techniques, will lead to a measurable increase in the risk of such tumors from that associated with 50 Gy of irradiation of the whole breast.

We found no significant increase in the degree of fibrosis in the treated breast among women who received an additional dose of radiation. However, a panel consisting of surgeons, radiation oncologists, and laypersons concluded that excellent or good cosmetic results were obtained in 86 percent of the patients in the standard-treatment group at three years, as compared with 71 percent of those in the additional-radiation group. 'The additional dose was, however, not the only factor with a negative influence on the cosmetic outcome. Other factors included the location of the primary tumor, the size of the tumor, the volume of tissue excised, and any complications of surgery. [21,22] A 5-year follow-up period is too short to allow a definitive analysis of late effects of radiation, [35] and a further analysis will be performed at 10 years. The reduction in the incidence of local recurrences associated with the additional dose far outweighs the slight increase in the poorer cosmetic outcome, especially in patients younger than 50 years of age.

\section{Acknowledgements}

Supported by grants (5R10-CA11488 through 5U10-CA11488-30) from the National Cancer Institute. The content of the article is solely the responsibility of the authors and does not necessarily reflect the official views of the National Cancer Institute.

We are indebted to the following persons for their help in the concept and design of the trial and their active participation: E. van der Schueren, Leuven, Belgium; B. Pierquin, Paris; R.P. Müller, Cologne, Germany; J. Kurtz, Geneva; D. Morgan, Nottingham, United Kingdom; J.B. Dubois, Montpellier, France; E. Salamon, Namur, Belgium; R.O. Mirimanoff, Lausanne, Switzerland; J.W.H. Leer, Leiden, the Netherlands; M. Bolla, Grenoble, France; A. Kuten, Haifa, Israel; A. Renaud, La Louvière, Belgium; U. Schulz, Krefeld, Germany; P.C.M. Koper, Rotterdam, the Netherlands; D. Van den Weyngaert, 
Antwerp, Belgium; G.A. Storme, Brussels, Belgium; G.H.M. Calitchi, Creteil, France; W. Budach, Berlin, Germany; S. Roth, Düsseldorf, Germany; M. Poulsen, Brisbane, Australia; M.A. Dominguez, Pamplona, Spain; E. Monpetit, Vannes, France; F. Kovner, Tel Aviv, Israel; A. Biete Sola, Barcelona, Spain; P. Calvo, Madrid, Spain; and C. Vrieling, Amsterdam, the Netherlands; and to J.L. Peterse for pathology review.

\section{References}

1. Sarrazin $\mathrm{D}, \mathrm{Le} M \mathrm{MG}$, Arriagada $\mathrm{R}$, et al. Ten-year results of a randomized trial comparing a conserwative treatment to mastectomy in early breast cancer. Radiother Oncol 1989;14:177-84.

2. Blicherr-Toft $M$, Rose $C$, Andersen JA, er al. Danish randomized trial comparing breast conservation rherapy with mastectomy: six yeats of liferable analysis. In. Consensus development conference on the rreatment of early-stage breast cancer. Journal of the National Cancer Institute monographs. No. 11. Washingron, D.C.: Government Printing Office, 1992:19-25. (NIH publication no. 90-3187.)

3. Veronesi $U$, Salwadori $B$, Luini $A$, et al. Breast conserwation is a safe method in patients with small cancer of the breast: long-term results of three randomised trials on 1,973 patients. Eur J Cancer 1995;31A:1574-9.

4. Fisher B, Anderson S, Redmond CK, Wolraark N, Wickerham DL, Cronin WM. Reanalysis and results affer 12 years of follow-up in a randomized clinical trial comparing total mastectomy with lumpectomy with or without irradiation in the treatment of breast cancer. N Engl J Med 1995; 333:1456-61.

5. van Dongen JA, Voogd $A C$, Fentiman IS, et al. Long-term results of a randomized trial comparing breastconserving therapy with mastectomy: European. Organizarion for Research and Treatment of Cancer 10801 trial. If Natl Cancer Inst 2000;92:1143-50.

6. Early Breast Cancer Trialists" Collaborarive Group. Effects of radiotherapy and surgery in early breast cancer: an overview of the randomized trials. N Engl J Med 1995;333:1444-55. [Erratum, N Engl J Med 1996;334:1003.]

7. Forrest AB, Stewant H], Everington D, et al. Randomised controlled rrial of conservation therapy for breast cancer: 6-year analysis of the Scottish trial. Lancet 1996;348:708-13.

8. Veronesi U, Luini A, Del Vecchio $M$, et al. Radiocherapy after breastpreserving surgery in women with localized cancer of the breast. N Engl J Med 1993;328:1587-91.

9. Uppsala-Orebro Breast Cancer Study Group. Sector resection with or without postoperative radiorherapy for stage I breast cancer" a randomized rrial. J Narl Cancer Inst 1990;82:277-82.

10. Clark RM, Whetan T. Levine $\mathrm{M}_{3}$ et al. Randomized clinical trial of breast irradiation following lumpectomy and axillary dissection for nodenegative breast cancer: an update. I Natl Cancer Inst 1996;88:1659-64.

11. International Union against Cancer. TNM dinical dassification. In: Hermanek P, Sobin LH, eds. TNM classification of malignant rumors, 4 th ed. rew. Berlin, Germany: Springer-Verlag, 1987:95-6.

12. Prescribing, recording, and reporting with photon beam therapy, Report 50. Berhesda, Md.: International Commission of Radiation Units and Measurcments, September 1993.

13. Barrelink $\mathrm{H}$, Garavaglia $\mathrm{G}$, Johansson KA, et al. Quality assurance in conservative treatment of early breast cancer: report on a consensus meering of the EORTC Radiorherapy and Breast Cancer Cooperative Groups and the EUSOMA (European Society of Mastology). Radiother Oncol 1991;22:323-6.

14. Van Tienhoven G, Van Bree NAM, Mijnheer BJ, Bartelink H. Qualicy assurance of the EORTC arial 22881/10882: "assessment of the role of the booster dose in breast conserving therapy": the Dummy Run. Radiother Oncol 1991:22:290-8.

15. Hamers HP, Johansson KA, Venselaar JLM, de Brouwer P, Hansson U, Moudi C. Entrance and exit TL-dosimetry in the conservative treatment of breast cancer: a pilot study for the EORTC-Radiotherapy Cooperative Group. Radiother Oncol 1991;22:280-4.

16. Heukelom $S$, Lanson $J H$, wan Tienhoven $G$, Mijnheer BJ. In vivo dosimetry during tangential breast treatment. Radiother Oncol $1991: 22: 269.79$.

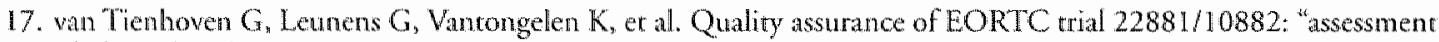
of the role of the booster dose in breast conserving therapy": the individual case review. Radiother Oncol 1992:24:Suppi:535, abstract. 
18. Pocock SJ, Simon R. Sequential treatment assignment with balancing for prognostic factors in the controlled dinical trial. Biometrics 1975;31:103-15.

19. Kaplan EL, Meier P. Nonparametric estimation from incomplete observations. I Am Stat Assoc 1958:53:457-81.

20. Cox DR, Oakes D. Analysis of survival dara. London: Chapnanan \& Hall, 1984.

21. Vrieling $C$, Collerte $L$, Fourquet $A$, et al. the influence of the boost in breast-conserving rherapy on cosmetic outcome in the EORTC "boost versus no boost" trial. Int J Radiat Oncol Biol Phys 1999;45:677-85.

22. Vrieling $C$, Colletre $L$, Fourquet $A$, et al. The influence of parient, tumor and treatmen factors on the cosmetic resules after breast-conserving therapy in the EORTC "boost vs no boost" trial. Radiother Oncol 2000; 55:219. 32.

23. The Steering Commitree on Clinical Practice Guidelines for the Care and Treatment of Breast Cancer. Breast radiotherapy afrer breast-conserving surgery. CMAJ 1998;158:Suppl 3:S35-\$42.

24. Romestaing $P$, Lehingue $Y$, Carrie $C$, et al. Role of a 10-Gy boost in the conservative treatment of early breast cancer: results of a tandomized clinical trial in Lyon, France. J Clin Oncol 1997:15:963-8.

25. de la Rochefordiere A, Asselain B, Campana F, et al. Age as prognostic factor in premenopausal breast carcinona. Lancet 1993;341:1039-43.

26. Abain KS, Allred DC, Clark GM. Breast cancer ontcome and predictors of outcome: are there age differentials? In: Breast cancer in young women. Journal of the National Cancer Institute monograplss. No. 16. Washington, D.C. Government Printing Office, 1994:35 42. (NIH publication no. 93-03559.)

27. Borger $J$, Kemperman $H$, Hart A, Peterse $\mathbb{H}$, wan Dongen J, Bartelink $H$. Risk factors in breast-conservatton therapy. \Clin Oncol 1994;12:653-60.

28. Voogd AC, Nielsen M, Pererse JL, er al. Differences in risk facrors for local and distant recurrence after breastconserving therapy or mastectomy for stage I and II breast cancer: pooled results of two large European randomized trials. ] Clin Oncol 2001;19:1688-97.

29. Elkhuizen PHM, van Slooren H], Clahsen PC, et all. High local recurrence risk after breast-conserwing therapy in node-negarive premenopausall breast cancer patients is grealy reduced by one course of perioperative chemotherapy: a European Organizarion for Research and Treatment of Cancer Breast Cancer Cooperative Group Srudy. I Clin. Oncol 2000;18:1075-83.

30. Owergaard $M_{n}$ Hansen PS, Overgaard I, et al. Postoperative radiotherapy in high-risk premenopausal women with breast cancer who receive adjuvant chemotherapy. N Engl J Med 1997:337:949-55.

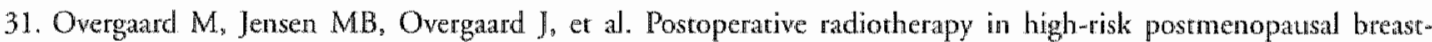
cancer patients given adjuvant tamoxifen: Danish Breast Cancer Cooperative Group DBCG $82 c$ randomised trial. Lancet 1999;353:1641-8.

32. Ragax J. Jackson SM, Le N, et al. Adjuvant radiorherapy and chemotherapy in node-positive premenopausal women with breast cancer. N Engl J Med 1997;337:956-2.

33. Huang J, Mackillop WJ. Increased risk of sof tissue sarooma after radiotherapy in women with breast carcinoma. Cancer 2001:92:172-80.

34. Shapiro CL, Recht A. Side effects of adjuvant trearment of breast cancer. N Engl J Med 2001;344:1997-2008.

35. Curran D, van Dongen JP, Aaronson NK, et al. Quality of life of earlystage breast cancer pritients treated with radical mastectony or breast-conserwing procedures: results of EORTC Trial 10801. Fur J Cancer 1998;34:307. 14. 


\section{CHAPTER 4}

The influence of the boost technique on local control in breast conserving treatment in the EORTC 'boost versus no boost' randomised trial.

Philip Poortmans, Harry Bartelink, Jean-Chaude Horiot, Henk Struikmans, Walter Van den Bogaert, Alain Fourquet, Jos Jager, Willem Hoogenraad, Parrick Rodrigus, Carla Warlam-Rodenhuis, Laurence Collette, Marianne Pierart, On behalf of the EORTC Radiotherapy and Breast Cancer Groups.

Radiotherapy and Oncology 72:25-33, 2004. 


\section{Abstract}

Background and purpose: The EORTC Trial 22881/10882 investigating the role of a boost dose in breast conserving therapy demonstrated a significantly better local control rate with the higher radiotherapy dose, especially in women younger than 50 years of age. This paper investigates the potential impact of the different boost techniques on local control and on fibrosis after breast conserving therapy.

Patients and methods: From 1989 to 1996, 2661 patients were randomised to receive a boost dose of $16 \mathrm{~Gy}$ to the primary tumour bed after microscopically complete tumorectomy and $50 \mathrm{~Gy}$ whole breast irradiation. The choice of the boost technique was left to the treating investigator. Treatment data were prospectively recorded as well as the clinical outcome in terms of local control and fibrosis. Sixty-three percent of the patients received a boost dose with fast electrons, $28 \%$ with photon beams and $9 \%$ with interstitial brachytherapy.

Results: At 5 years, local recurrences were seen in 74 of the 1635 patients who received an electron boost $(4.8 \%$, CI $3.6-5.9 \%)$, in 28 of the 753 patients who received a photon boost ( $4.0 \%, \mathrm{CI} 3.4-5.5 \%)$ and in 6 of the 225 patients after an interstitial boost (2.5\%, $\mathrm{Cl} 0.3-4.6 \%)$. The grade of fibrosis in the whole breast as well as at the primary tumour bed, as scored by the treating radiation oncologist, was similar in the three groups.

Conclusions: Although the three groups are of a rather unequal size, the results of the interstitial boost seem similar in terms of fibrosis and at least as good in terms of local control, despite a lower treatment volume and a longer overall treatment time.

Keywords: Breast cancer; Radiotherapy; Breast-conserving therapy; Brachytherapy; Interstitial boost; Electrons 


\section{Introduction}

Radiotherapy is useful to prevent cancer recurrence in the conservatively operated breast [6]. A dose-response relationship for local control was suggested in the retrospective analyses of clinical data published by several authors [1,5,20]. A large bulk of data, including trial 10801 conducted by the European Organisation for Research and Treatment of Cancer (EORTC) during the 1980s, demonstrated that boost doses in excess of $20 \mathrm{~Gy}$ following the administration of $50 \mathrm{~Gy}$ to the whole breast resulted in a poor cosmetic outcome and could hamper clinical and radiological examination during follow-up [19,24]. These observations motivated the EORTC to design a large randomised trial (EORTC 22881/10882) investigating the potential advantages and disadvantages of delivering a higher localised radiation dose to the tumour bed following conservative surgery for early breast cancer. The previous unfavourable experience with boost doses in excess of $20 \mathrm{~Gy}$ motivated the choice of a dose of $16 \mathrm{~Gy}$ for the new trial. An intensive quality assurance program was implemented to encourage uniformity of treatment delivery among centres [2]. The results after a median follow-up of 5.1 years were published earlier. They confirmed and strengthened the conclusions of a smaller French trial $[3,17]$.

According to the EORTC protocol, patients randomised to receive a boost dose received a supplementary dose of $16 \mathrm{~Gy}$ at the site of the original tumour. As the effect of the type of boost on the outcome was uncertain, all three commonly used techniques were allowed for delivering the boost dose: external photon irradiation, external electron irradiation and interstitial irradiation. The present report describes the influence of the boost technique on local control and on fibrosis after breast conserving therapy in the group of patients receiving a boost in the framework of a large prospective randomised multi-centre trial.

\section{Patients and methods}

From 1989 to 1996, EORTC Trial 22881/10882 accrued 5569 stage I and II breast cancer patients whom had undergone a macroscopically complete surgical removal of the breast tumour and an axillary dissection. The 5318 patients whose tumours had been microscopically completely excised (as derived from the local pathology examination) were randomised to receive 50 Gy whole breast irradiation with $(n=2661)$ or without $(n=2657)$ a boost of 16 Gy to the tumour bed. The randomisation was stratified for age, menopausal status, presence of intraductal component, clinical tumour size, dinical nodal status, and institure. Patients with clinical stage $\mathrm{T} 1-2, \mathrm{NO}-1$, and $\mathrm{MO}$ breast cancer 
Type of Boost

\begin{tabular}{|c|c|c|c|c|c|}
\hline & $\begin{array}{c}\text { Unknown } \\
(\mathbb{N}=48)\end{array}$ & $\begin{array}{l}\text { Electrons } \\
(\mathrm{N}=1635)\end{array}$ & $\begin{array}{l}\text { Photons } \\
(\mathrm{N}=753)\end{array}$ & $\begin{array}{c}\text { Interstitial } \\
(\mathrm{N}=225)\end{array}$ & $\begin{array}{c}\text { Total } \\
(\mathrm{N}=2661)\end{array}$ \\
\hline & $\mathbb{N}(\%)$ & $N(\%)$ & $\mathrm{N}(\%)$ & $\mathrm{N}(\%)$ & $\mathbf{N}(\%)$ \\
\hline \multicolumn{6}{|l|}{$\overline{\text { Agre }}$} \\
\hline$<=40$ & $8(16.7)$ & $149(9.1)$ & $50(6.7)$ & (6.2) & $221(8.3)$ \\
\hline $41-50$ & $8(16.7)$ & $411(25.1)$ & $187(248)$ & $63(28,0)$ & $669(25.1)$ \\
\hline $51-60$ & $13(27.1)$ & $520(31.8)$ & $256(34.0)$ & $711(31.6)$ & $860(32.3)$ \\
\hline 61.70 & $19(39.6)$ & $555(33.9)$ & $260(34.5)$ & $77(34.2)$ & $911(34.2)$ \\
\hline Median & 57.8 & 54.6 & 55.7 & 54.4 & 54.8 \\
\hline Range & $28.3-75.2$ & $26.4-78.8$ & $25.6 \cdot 73.8$ & $27.3-72.1$ & $25.6-78.8$ \\
\hline \multicolumn{6}{|l|}{ Clinical T-staging } \\
\hline$T 1$ & $23(47.9)$ & $886(54.2)$ & $363(48.2)$ & $101(44.9)$ & $1373(51.6)$ \\
\hline 12 & $25(52.1)$ & $747(45.7)$ & $389(51.7)$ & $120(53.3)$ & $1281(48.1)$ \\
\hline T3 & $0(0.0)$ & $2(0.1)$ & $\llbracket(0.1)$ & $4(1.8)$ & $7(0.3)$ \\
\hline \multicolumn{6}{|l|}{ Clinicall N-staging } \\
\hline No & $39(81.3)$ & $1462(89.4)$ & $676(89.8)$ & $206(91.6)$ & $2383(89.6)$ \\
\hline $\mathrm{NI}$ & 1. $(2.1)$ & $45(8.9)$ & $47(6.2)$ & $16(7.1)$ & $209(7.8)$ \\
\hline Unknown & $8(16.7)$ & $28(1.8)$ & $30(4.0)$ & $3(1.3)$ & $69(2.6)$ \\
\hline \multicolumn{6}{|l|}{ Renexcision performed } \\
\hline $\mathrm{No}$ & $30(62.5)$ & $1241(75.9)$ & $541(71.8)$ & $179(79.6)$ & $1991(74.8)$ \\
\hline Yes & $11(22.9)$ & $393(24,0)$ & $212(28.2)$ & $46(20.4)$ & $662(24.9)$ \\
\hline Unknown & $7(14,6)$ & $1(0.1)$ & $0(0.0)$ & $0(0.0)$ & $8(0.3)$ \\
\hline \multicolumn{6}{|l|}{ Pathological T-measurement } \\
\hline$<10 \mathrm{~mm}$ & $10(20.8)$ & $390(23.9)$ & $183(24.3)$ & $52(23.1)$ & $635(23.9)$ \\
\hline $110-20$ & $26(54.2)$ & $912(55.7)$ & $393 .(52.2)$ & $120(53.3)$ & $1451(54.5)$ \\
\hline$>20$ & $2(4.2)$ & $305(18.7)$ & $1.59(21.1)$ & $47(20.9)$ & $513(19.3)$ \\
\hline Unknown & $10(20.8)$ & $28(1.7)$ & $18(2.4)$ & $6(27)$ & $62(2.3)$ \\
\hline \multicolumn{6}{|l|}{ Nb Positive Nodes } \\
\hline None & $35(72.9)$ & $1283(78.5)$ & $594(78.9)$ & $178(79.1)$ & $2090(78.5)$ \\
\hline $1-3$ & $5(10.4)$ & $272(16.6)$ & $133(17.7)$ & $39(17.3)$ & $449(16.9)$ \\
\hline $4+$ & $\mathbb{1}(2 . \mathrm{I})$ & $70(4.3)$ & $23(3.1)$ & $8(3.6)$ & $102(3.8)$ \\
\hline Unknown & $7(14.6)$ & $10(0.6)$ & $3(0.4)$ & $0(0.0)$ & $20(0.8)$ \\
\hline \multicolumn{6}{|l|}{ Histologic type } \\
\hline Invasive ductal carcinoma & $33(68.8)$ & $1356(82.9)$ & $625(83.0)$ & $184(81.8)$ & $2198(82.6)$ \\
\hline Invasive lobular carcinoma & $2(4.2)$ & $144(8.8)$ & $54(7.2)$ & $19(8.4)$ & $219(8.2)$ \\
\hline Other & $6(12.6)$ & $134(8.4)$ & $74(9.9)$ & $22(9.8)$ & $236(8.8)$ \\
\hline Unknown & $7(14.6)$ & $1(0.1)$ & $0(0.0)$ & $0(0,0)$ & $8(0.3)$ \\
\hline \multicolumn{6}{|l|}{ Estrogen receptor status (ER) } \\
\hline Negatiwas & 9) (18.8) & $329(20.1)$ & $156(20.7)$ & $34(15.1)$ & $528(19.8)$ \\
\hline Positive & $7(35.4)$ & $872(53.3)$ & $396(52.6)$ & $124(55.1)$ & $1409(53.0)$ \\
\hline Unknown & $22(45.9)$ & $434(26.6)$ & $201(26,7)$ & $67(29.8)$ & $724 .(27.2)$ \\
\hline \multicolumn{6}{|l|}{ Progestronc receptor scatus (PR) } \\
\hline Negative & $4(8.3)$ & $400(24.5)$ & $175(23.2)$ & $46(20,4)$ & $625(23.5)$ \\
\hline Positive & $19(39.6)$ & $715(43.7)$ & $349(46.3)$ & $104(46.2)$ & $1187(44.6)$ \\
\hline Unknown & $25(52.1)$ & $520(31.8)$ & $229(30.4)$ & $75(33.3)$ & $849(31.9)$ \\
\hline
\end{tabular}

Table 1. Pationt and tumour chanacteristics of the study population, divided per boost technique 
were eligible for the trial. The patients were treated in 31 institutions from nine countries. Informed consent was obtained according to EORTC guidelines and the local and national rules of the participating centres [3]. This report concerns the 2661 patients with a complete excision of the primary tumour who were randomised to receive a boost dose of $16 \mathrm{~Gy}$ to the primary tumour bed. Patient characteristics are displayed in Table 1 .

In the absence of adjuvant chemotherapy, radiotherapy was to start within 9 weeks following lumpectomy. For patients receiving adjuvant chemotherapy, a delay of up to 6 months before beginning breast irradiation was allowed. Whole-breast irradiation was given using two tangential megavoltage photon (high-energy $\mathrm{X}$-ray or tele-cobalt) beams. A close of $50 \mathrm{~Gy}$ in 5 weeks, with a dose per fraction of $2 \mathrm{~Gy}$, was to be specified at the intersection of the central axes of the beams $[2,11]$. The target area for the boost was the original site of the primary tumour, with a safety margin of $1.5 \mathrm{~cm}$ around the primary tumour after microscopic complete excision and of $3 \mathrm{~cm}$ in case of invasive cancer with an extensive DCIS component. The boost dose of $16 \mathrm{~Gy}$ was specified at the centre of the tumour excision area. For electrons, the dose was specified at the depth of Dmax with the $85 \%$ isodose encompassing the target volume. In case of an interstitial boost, $15 \mathrm{~Gy}$ was prescribed according to the Paris system. This dose was fixed and independent of the dose delivered to this area during the external irradiation of the whole breast. For the localisation of the primary tumour site, information was used from clinical examination before and after surgery, preoperative mammography, surgical reports describing the tumour site, surgical marker clips if present and the scar. The choice of the boost technique of boost delivery was free and could be individualised based on experience and on patient and tumour specific parameters. The majority of the patients randomised to receive the boost dose $(1635 / 2661=63 \%)$ was treated with eight external-beam fractions of 2 Gy each with fast electrons. $753(29 \%)$ patients were treated with eight fractions of tangential or wedged oblique photon fields, and $225(9 \%)$ were given a boost of 15 Gy with an iridium-192 implant delivered at a dose rate of 10 Gy per 24 h. For 22 patients, data on the boost technique are missing and 26 patients did not receive a boost.

Ar each follow-up visit, the radiation oncologist scored the grade of fibrosis based on physical examination, for the whole breast as well as for the primary tumour bed only. The scoring was on a 4-point scale (none-minor-moderate-severe). The worst score reported over the follow-up is used for the present report.

An extensive quality assurance program was implemented to ensure that breast irradiation was carried out according to acceptable standards in all centres $[9,15,21,22]$. This program involved a 'dummy run' procedure to check dose prescription and dose calculations, as well as site visits to verify accuracy of data. Moreover, as part of the 
EORTC Radiotherapy Group Quality Assurance Program, a team of physicists verified beam calibration in the participating centres and a trial specific thermoluminescent dosimetry program was set up. If a major deviation between dose calculation and dose delivery was detected, a local audit was held to identify the reason. A central pathology review was performed at the Netherlands Cancer Institure.

At the time of this report, the median follow-up was 5.11 years (maximum 10.2 years). In the 2661 patients with a microscopically complete resection randomised to receive a boost, 234 deaths had occurred, of which 175 (74.8\%) were due to breast cancer. All analyses presented in this report are descriptive. Local failure rates were estimated using the Kaplan-Meier technique.

\section{Results}

Local recurrence was observed in 109 of the 2661 patients randomised to receive an additional $16 \mathrm{~Gy}$ boost following $50 \mathrm{~Gy}$ whole-breast irradiation. This corresponds to a 5-year actuarial local recurrence rate of $4.3 \%(95 \% \mathrm{CI}: 3.8-4.7 \%) .48 \%$ of the local recurrences occurred in the primary tumour bed, $9 \%$ in the scar, $28 \%$ outside the original tumour area, and $14 \%$ were diffuse.

The local recurrence rates by boost technique are displayed in Table 2 and in Fig. 1. After a median follow-up of 5.11 years, the apparent difference in local control rate between the different boost techniques is not statistically significant and the precision of the curves is not high due to the less number of events and patients in some of the groups.

As age was, together with the use of a boost dose, by far the most important prognostic factor for local control, we analysed the use of the different boost techniques in four different age groups. No differences could be found, thereby excluding age as a potential confounding factor in the comparison of the outcome of treatment across the different boost techniques (Table 3). The dose delivered at the centre of the primary tumour bed and the volume of the boost are displayed in Table 4 for the three different boost techniques. The median boosted volume was smallest with the interstitial technique $(60 \mathrm{cc})$, more than twice as large with an electron boost $(144 \mathrm{cc})$ and nearly five times as large with photons $(288 \mathrm{cc}$ ). The overall treatment time is also displayed in Table 4: whereas the external boost started for most patients without any delay following whole breast irradiation, a median delay of 18 days was noted between the end of external beam. irradiation and the administration of the interstitial boost. This resulted in a considerably longer overall treatment time in this group of patients ( 54 versus 48 days). 
Type of Boost

\begin{tabular}{|c|c|c|c|c|c|}
\hline & & & & & \multirow[b]{2}{*}{$\begin{array}{c}\text { Total } \\
(\mathrm{N}=2661)\end{array}$} \\
\hline & $\begin{array}{c}\text { Unkanown } \\
(\mathrm{N}-48)\end{array}$ & $\begin{array}{l}\text { Elecrrons } \\
(\mathrm{N}=1635)\end{array}$ & $\begin{array}{l}\text { Photonas } \\
(\mathrm{N}=753)\end{array}$ & $\begin{array}{c}\text { Interstitial } \\
(\mathrm{N}=225)\end{array}$ & \\
\hline \multicolumn{6}{|l|}{ Local failures } \\
\hline $\mathbf{n}$ & $\mathbb{1}$ & 74 & 28 & 6 & 109 \\
\hline$\%$ & & $47 \%$ & $4.0 \%$ & $25 \%$ & 4,306 \\
\hline $95 \% \mathrm{CI}$ & & $(3.6-5.9 \%)$ & $(2.4-5.5 \%)$ & $(0.3-4,60)$ & $(3.9-4700)$ \\
\hline
\end{tabular}

Table 2. 5-year local failure rates, diwided by boost technique

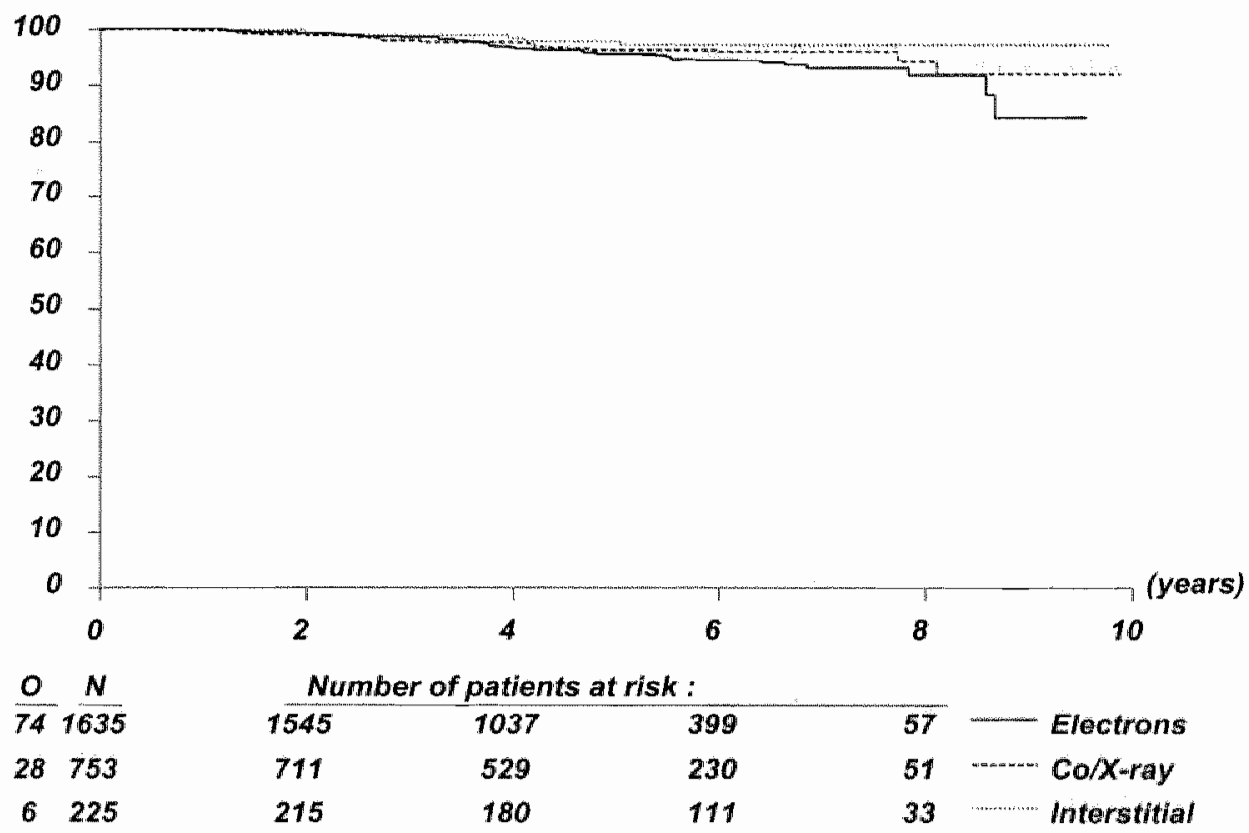

Figure I. Local cantrol divided by boost technigue (Kaplan-Meier)

\begin{tabular}{|c|c|c|c|c|c|}
\hline & & $\mathrm{Ag}$ & refirs) & & \\
\hline & $\begin{array}{c}c=40 y \\
(N=221)\end{array}$ & $\begin{array}{c}41-50 \\
(N=669)\end{array}$ & $\begin{array}{c}51-60 \\
(\mathrm{~N}=860)\end{array}$ & $\begin{array}{c}61-70 \\
(\mathrm{~N}=911)\end{array}$ & $\begin{array}{c}\text { Total } \\
(N=2661)\end{array}$ \\
\hline Type of boost & $N \%$ & $\mathrm{~N} \%$ & $\mathbb{N} \%$ & $N \%$ & $N \%$ \\
\hline Electroms & $1.49(67.4)$ & $411(61.4)$ & $520(60,5)$ & $555(60.9)$ & $1635(61.4)$ \\
\hline Photons & $50(22.61)$ & $187(28.0)$ & $256(29.8)$ & $260(28.5)$ & $753(28.3)$ \\
\hline Interstitial & $14(6.3)$ & $63(9.4)$ & $71(8.3)$ & $77(8.5)$ & $225(8.5)$ \\
\hline Unknown & $8(3.6)$ & $8(1.2)$ & $13(1.5)$ & $19(2.1)$ & $48(1.8)$ \\
\hline
\end{tabular}

Table 3. Boost techniques, divided by age group 


\section{Wlectrons}

\begin{tabular}{|c|c|c|c|}
\hline \multicolumn{4}{|l|}{ Boost dose (Gx) } \\
\hline Med han & 16.0 & 16.0 & 15.0 \\
\hline Range & $60-260$ & 70.262 & $12.7-25.0$ \\
\hline \multicolumn{4}{|c|}{ Boost targer wollunte $(\mathrm{sc})$} \\
\hline Med han & $\therefore 1440$ & 280.0 & 60,0 \\
\hline Range & $22.0-940.0$ & $48.0-1386.0$ & $10.0-240.0$ \\
\hline \multicolumn{4}{|c|}{ Time from end of WB l no boost (days) } \\
\hline Median & 1.0 & 1.0 & 18.0 \\
\hline Range: & $-25.0-95.0$ & $-9.0-19.0$ & $1.0-54.0$ \\
\hline \multicolumn{4}{|c|}{ Total trearment tine (WHI + Boost) (days) } \\
\hline Median & 48.0 & 48.0 & 540 \\
\hline Range & $37.0-141.0$ & $39.0-81.0$ & $35.0-94.0$ \\
\hline
\end{tabular}

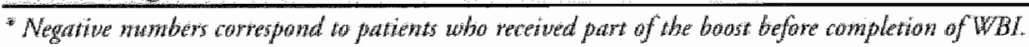

Table 4. Dose, wolnme and time characteristics of the different boost techniques.

WBI = whole breast imadiation.

As shown in Tables 5 and 6 and in Figs. 2 and 3, the occurrence and the grade of fibrosis in the whole breast as well as in the boost area was similar for the three boost techniques. Over all, $12 \%$ of the patients developed moderate to severe fibrosis in the whole breast and $25 \%$ at the site of the primary tumour.

\section{Type of Boost}

\begin{tabular}{|c|c|c|c|c|c|}
\hline & $\begin{array}{c}\text { Unknown } \\
(\mathrm{N}=48)\end{array}$ & $\begin{array}{l}\text { Electrons } \\
(\mathrm{N}=1635)\end{array}$ & $\begin{array}{l}\text { Photons } \\
(\mathrm{N}=753)\end{array}$ & $\begin{array}{c}\text { Interstitial } \\
(\mathrm{N}=225)\end{array}$ & $\begin{array}{c}\text { Total } \\
(\mathrm{N}=2661)\end{array}$ \\
\hline & $\mathrm{N}(\%)$ & $N(\%)$ & $\mathbf{N}(\%)$ & $\mathbf{N}(\%)$ & $\mathbf{N}(\%)$ \\
\hline \multicolumn{6}{|c|}{ Palpable fibrosis in whole breast } \\
\hline None & $14(292)$ & $824(50.4)$ & $383(50.9)$ & $118(52.4)$ & $1339(50.3)$ \\
\hline Minor & $12(25.0)$ & $575(35.2)$ & $243(32.3)$ & $78(34.7)$ & $908(34.1)$ \\
\hline Moderate & $2(4.2)$ & $1480.1)$ & $97(12.9)$ & $14(6.2)$ & $261(9.8)$ \\
\hline Severe & $0(0.0)$ & $14(0.9)$ & $16(2.1)$ & $2(0.9)$ & $32(1.2)$ \\
\hline Unknown & $20(41.7)$ & $74(4.5)$ & $14(1.9)$ & $13(5.8)$ & $121(4.5)$ \\
\hline of with fibrosis at 5 yetrs & & $47.2 \%$ & $48.2 \%$ & $39.6 \%$ & Logrank P \\
\hline$(95 \% \mathrm{CD})$ & & $(44,5-49.9)$ & $(44.4-52.1)$ & $(33.8-46.3)$ & 0.013 \\
\hline
\end{tabular}

Table 5. Grude of fibrosis in the whole breast, divided by boost technigue 
Type of Boost

\begin{tabular}{|c|c|c|c|c|c|}
\hline & $\begin{array}{l}\text { Unknown } \\
(\mathrm{N}=\mathbf{4 8})\end{array}$ & $\begin{array}{l}\text { Electrons } \\
(\mathrm{N}=1635)\end{array}$ & $\begin{array}{l}\text { Photons } \\
\text { (N-753) }\end{array}$ & $\begin{array}{c}\text { Interstitial } \\
(\mathrm{N}=225)\end{array}$ & $\begin{array}{c}\text { Total } \\
(\mathrm{N}=2661)\end{array}$ \\
\hline & $\mathbf{N}(\%)$ & $N(\%)$ & $N(0)$ & $\mathbf{N}(\%)$ & $N(\%)$ \\
\hline \multicolumn{6}{|c|}{ Balpable fibrosis in wholle breast } \\
\hline None & $15(31.3)$ & $533(32,6)$ & $285(37.8)$ & $59(26.2)$ & $892(33.5)$ \\
\hline Minor & $5(10,4)$ & $661(40.4)$ & $256(34.0)$ & 91. $(40.4)$ & $1013(38.1)$ \\
\hline Moderate & $4(8.3)$ & $320(196)$ & $165(21,9)$ & $51(227)$ & $540(20.3)$ \\
\hline Severe & $0(0.0)$ & $46(2.8)$ & $33(4.4)$ & $10(4,4)$ & $82(3.3)$ \\
\hline Unlknown & $24(50.0)$ & $75(4.6)$ & $14(1.9)$ & $14(6,2)$ & $127(4.8)$ \\
\hline of with fibrosis at 5 years & & $65.6 \%$ & $61.4 \%$ & $67.2 \%$ & Logrank $\mathrm{P}$ \\
\hline$(95 \% \mathrm{CI})$ & & $(63,0-68.2)$ & $(57.6-65.1)$ & $(60.6-73.9)$ & 0.672 \\
\hline
\end{tabular}

Table 6. Grade of fibrosis at the primary tumown bed, divided by boost tedmigne

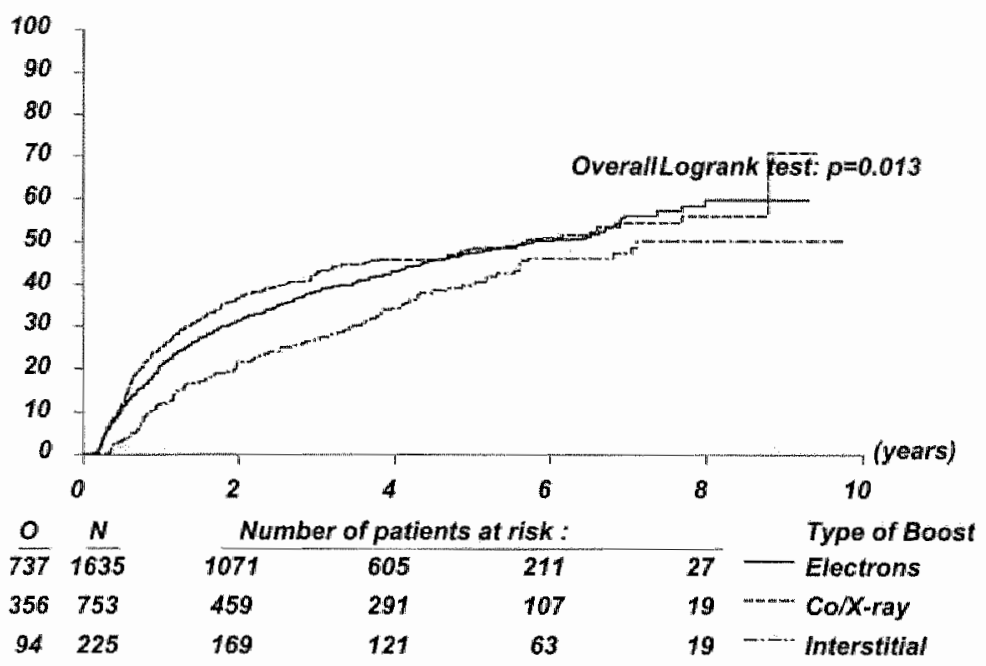

Figure 2. Thme to the development of fibrosis (any grade) in the whole breast (Kaplan-Meter)

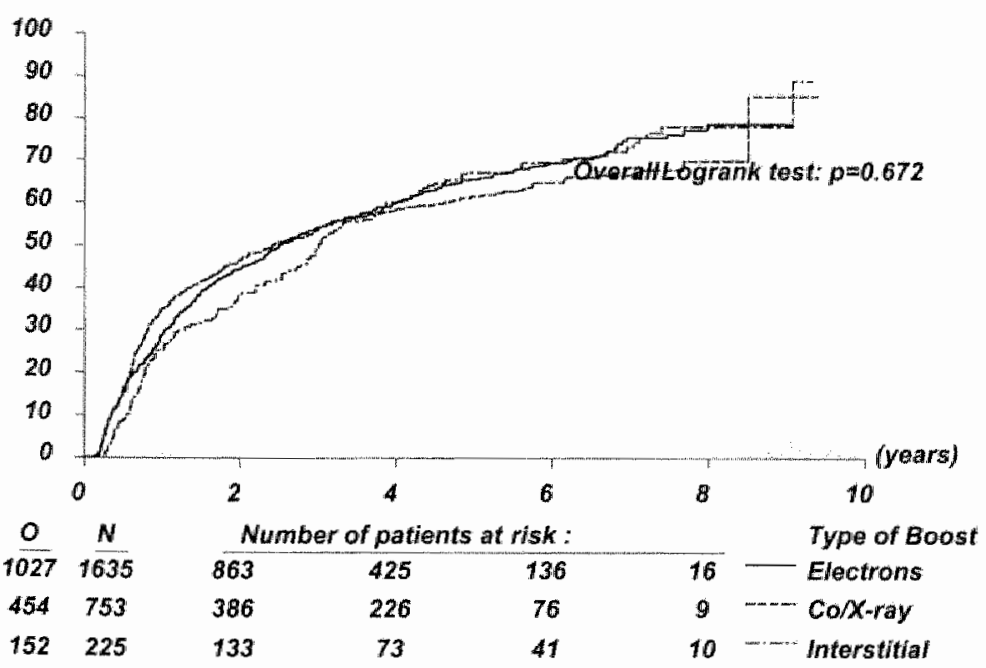

Figure 3. Time to the development of fibrosis (any grade) in the boost area (Kaplan-Meier) 
We analysed the use of the different boost techniques over time and over the major participating countries (Tables 7 and 8 ). In the Netherlands, which was by far the major contributor to the study, an electron boost was used in $59 \%$ of the parients, a photon boost in $31 \%$ and interstitial therapy in $8 \%$. In France, the latter technique was applied in $11 \%$ of the patients. Belgium was the country where the interstitial therapy boost was used most $(16 \%)$ and where a photon boost was rarely used. Over the time span of this trial, the relative use of an external photon beam technique and especially the interstitial therapy boost technique decreased gradually from 30 to $25 \%$ and from 14 to $4 \%$, respectively, compensated by a rise in the use of electrons from 54 to $69 \%$.

\begin{tabular}{ccccc} 
& $\begin{array}{c}\text { Unknown } \\
(\mathbf{N}=48)\end{array}$ & $\begin{array}{c}\text { Electrons } \\
(\mathbf{N}=1635)\end{array}$ & $\begin{array}{c}\text { Photons } \\
(\mathbf{N}=753)\end{array}$ & $\begin{array}{c}\text { Interstitial } \\
(\mathbf{N}=225)\end{array}$ \\
\cline { 2 - 5 } & $\mathbf{N}(\%)$ & $\mathbf{N}(\%)$ & $\mathbf{N}(\%)$ & $\mathbf{N}(\%)$ \\
\hline COUNTRY & & & & \\
\hline The Netherlands & $24(1.9)$ & $747(59.4)$ & $390(31.0)$ & $97(7.7)$ \\
\hline France & $13(2.3)$ & $311(55.5)$ & $172(90.7)$ & $64(11.4)$ \\
\hline Belgium & $0(0.0)$ & $291(75.0)$ & $36(9.3)$ & $61(15.7)$ \\
\hline Others & $11(2.4)$ & $286(62.9)$ & $155(34.1)$ & $3(0.7)$ \\
\hline
\end{tabular}

Table 7. Boost techriques, divided by country

\begin{tabular}{ccccc} 
& $\begin{array}{c}\text { Unknown } \\
(\mathbf{N}=48)\end{array}$ & $\begin{array}{c}\text { Electrons } \\
(\mathbf{N}=1635)\end{array}$ & $\begin{array}{c}\text { Photons } \\
(\mathbf{N}=753)\end{array}$ & $\begin{array}{c}\text { Interstitial } \\
(\mathbf{N}=225)\end{array}$ \\
\cline { 2 - 5 } & $\mathbf{N}(\%)$ & $\mathbf{N}(\%)$ & $\mathbf{N}(\%)$ & $\mathbf{N}(\%)$ \\
\hline Period & & & & \\
\hline $1989-1992$ & $22(2.1)$ & $562(53.8)$ & $316(30.2)$ & $145(13.9)$ \\
\hline $1993-1994$ & $12(1.2)$ & $677(65.0)$ & $296(28.4)$ & $56(5.4)$ \\
\hline $1995+$ & $14(2.4)$ & $396(68.9)$ & $141(24.5)$ & $24(4.2)$ \\
\hline
\end{tabular}

Table 8. Boost techniques, divided by vime period

\section{Discussion}

EORTC trial 22881/10882 demonstrated that a higher radiation dose significantly reduced the incidence of local recurrences after breast conserving therapy, while only slightly impairing the cosmetic outcome. The clinical benefit of the extra radiation dose was especially significant and clinically relevant in younger patients. A longer follow-up is needed to evaluate the benefit of the treatment in terms of distant-metastases free and overall survival and long-term side effects. These results so far demonstrate that, at least for patients younger than 50 years, whole breast irradiation to $50 \mathrm{~Gy}$ should be followed 
by a boost dose of $16 \mathrm{~Gy}$.

The present analysis indicated that the technique of the boost delivery did not seem to have an impact on local control nor on treatment induced fibrosis. Longer follow-up is needed to assess whether or not the observed small trend in favour of the interstitial boost technique for local control will hold. But, as we report on non-randomised patient groups and as the median follow-up in the interstitial boost subgroup is considerably longer than in the other groups, no definitive conclusions regarding this question will be reached with this study and the results should be regarded as exploratory in nature. However, no major differences in patient characteristics were seen between the three groups, probably because of the large number of patients involved in the trial as a whole (Table 1). Longer follow-up is also needed for the definitive assessment of long-term side effects of the treatment including the development of fibrosis.

Frazier and colleagues found no significant differences in local recurrence rates or cosmetic outcome in their institutional based retrospective analysis of 552 patients with 563 stage I and II breast cancers, whether the boost was given with electrons ( $n=232$ ); photons $(n=15)$ or an interstitial implant $(n=316)$ [7]. Perez and colleagues retrospectively reviewed 701 patients treated with breast conserving therapy over a long period of time (1967-1990). Local tumour control, disease-free survival, cosmetic results and morbidity were equal for 449 patients treated with an electron boost and for 129 patients who received an interstitial boost [14]. Resch found a favourable actuarial 10year local control rate of $96.1 \%$ in 410 patients treated at one institution with BCT including interstitial therapy [16]. In an overview article, Hammer and colleagues concluded that careful selection of treatment procedures for specific subgroups of patients as well as refinement in surgical procedures and radiotherapy techniques may be useful to improve the clinical and cosmetic results of breast conserving therapy. They proposed to compare the different boost techniques in a prospective trial [10]. The safety of the three different boost techniques in terms of local control and fibrosis is confirmed in our large multi-centre international trial, with a possible advantage of the interstitial therapy boost technique. Although the boosted volume was significantly smaller and the overall treatment time markedly longer, the local control in this group was at least as good as with the other boost techniques. This might be explained by a favourable radiobiological effect of continuous low dose rate interstitial therapy on proliferating breast cancer cells, as compared to the effect of separate fractions of 2 Gy spread over nearly 2 weeks. Another explanation can be an effect of the higher doses, which are part of the heterogeneous dose distribution within the interstitial boost target volume, specified according to the Paris system. 
In terms of cosmesis, the most important variables which negarively affect the cosmetic outcome, are an inferior tumour locarion, a larger excised volume, post-operative breast complications and the delivery of a boost dose $[25,26]$. We reported earlier that at 5 years median follow-up the delivery of a boost dose did not lead to significant differences in the grade of fibrosis in the whole breast compared to the patients who did only receive whole breast irradiation [3]. About the impact of the boost technique itself, reports in the literature are conflicting $[4,10,18,23,27]$. This might relate to a possible influence of the technical aspects of especially the interstitial therapy techniques $[8,12,13]$. If interstitial therapy is used properly for deeply seated target volumes, target volume coverage will be more conformal by interstitial therapy than by electron beams or two tangential photon. beams. This difference is less evident of course for more superficially located target volumes. The fibrosis as measured in the whole breast and at the primary tumour site was similar with the different boost techniques.

The fibrosis at the primary tumour bed was more obvious than in the whole breast. This can be explained by the higher dose delivered to the primary tumour bed, leading to localised fibrosis without a substantial influence on the remaining breast tissue.

The relative use of the different boost techniques varied considerably between countries. This is probably based on personal experience of the treating radiation oncologists with a broad experience in the treatment of breast cancer and on local variations in technical choices proposed by major teaching hospitals. From the late $1980 \mathrm{~s}$ to the mid-1990s, the use of an interstitial boost decreased by about 60\% while the electron boost further gained popularity. This can be explained by the modernisation of the external radiotherapy equipment in the early 1990 s when most of the older cobalt and single lowenergy photon linear accelerators were replaced by modern higher energy linear accelerators allowing access to a wider range of electron beam energies. Moreover, an increasing burden of radiation protection restrictions reduced the practice of manual afterloading inrerstitial therapy, whicln was widely replaced by the use of an electron beam boost. Nowadays, in several centres the application of the interstitial boost technique is restricted to deeply seated target volumes in large breasts because of its unrivalled dose distribution in this situation, provided that the boost target volume can be determined reliably.

\section{Conclusions}

The large randomised EORTC 22881 trial clearly demonstrated the benefit of a 16 Gy boost dose after complete resection and whole breast irradiation to $50 \mathrm{~Gy}$, especially in 
patients younger than 50 years. Notwithstanding the huge number of patients entered in our study, definite condusions on the optimal choice for a boost technique cannot be drawn because this question was not randomised and the comparison therefore only descriptive in nature. The interstitial boost technique seems similar in terms of fibrosis and at least as good in terms of local control, despite the considerably lower treatment volume and the longer overall treatment time. This might be explained by radiobiological factors and the heterogeneity of the dose distribution within the interstitial boost volume. At this moment, a new international randomised multicenter trial is being prepared to investigate the effect of further increasing the boost dose to $26 \mathrm{~Gy}$ in patients up to 50 years of age with the use of modern interstitial or conformal external radiotherapy.

\section{Acknowledgements}

We acknowledge and thank sincerely the following for their help in the concept, the design and the active participation of the trial: van der Schueren $\mathrm{E}$, Leuven (B); Pierquin B, Paris (F); Müller RP, Cologne (D); Kurtz J, Geneva (CH); Morgan D, Nottingham (GB); Dubois JB, Montpellier (F); Salamon E, Namur (B); Mirimanoff RO, Lausanne (C); Leer JWH, Leiden (NL); Bolla M, Grenoble (F); Kuten A, Haifa (I); Renaud A, La Louviere (B); Schulz U, Krefeld (D); Koper PCM, Rotterdam (NL); Van den Weyngaert D, Antwerpen (B); Storme GA, Brussels (B); Calitchi GHM, Creteil (F); Budach W, Berlin (D); Roth S, Dusseldorf (D); Poulsen M, Brisbane (A); Dominguez MA, Pamplona (E); Monpetit E, Vannes (F); Kovner F, Tel Aviv (I); Biete Sola A, Barcelona (E); and Calvo F, Madrid (E).

This publication was supported by grants number 3U10 CA11488 through 18S1 through 5U10 CA11488-33 from the National Cancer Institute (Bethesda, Maryland, USA). Its content is solely the responsibility of the authors and does not necessarily represent the official views of the National Cancer Institute. The data management for the Dutch patients was supported by a grant from the Dutch cancer foundation.

\section{References}

1. Arriagada R, Mouriesse H, Sarrazin D, Clark RM, Deboer G. Radiotherapy alone in breast cancer. I. Analysis of tumor parameters, tumor dose, and local control: the experience of the Gustave-Roussy Institure and the Princess Margaret Hospital. Int J Radiat Oncol Biol Phys 1985;11:1751-7.

2. Bartelink $\mathrm{H}$, Garavaglia $\mathrm{G}$, Johansson $\mathrm{KA}$, er al. Quality assurance in conservative treatment of carly breast cancer. Wheport on a consensus meering of the EORTC Radiotherapy and Breast Cancer Cooperative Groups and the EUSOMA (European Society of Mastology). Radiother Oncol 1991;22:323-6.

3. Bartelink $\mathrm{H}$, Horior $\mathrm{J}-\mathrm{C}$, Poortmans $\mathrm{P}$, et al. Recurrence rates after treatment of breast eancer with standard radiotherapy with or without additional radiation. N Engl J Med 2001;345:1378-87. 


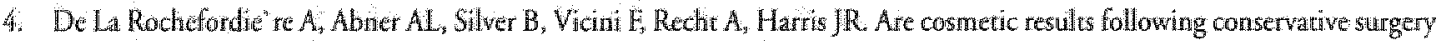
and radiation therapy for early breast cancer dependent on technique? Int J Radiat Oncol Biol Phys 1992;23:925-31.

5. Denharn JW. The radiation dese-response relarionship for control of primary breast cancer. Radionter Oncol $1986,7: 107-23$.

6. Early Breast Cancer 'Trialisis' Collaborative Group, Effecs of radiotherapy and surgery in carly breast cancer. An overview of the randonized trials. N Engl J Med 1995;333:1444-55.

7. Frazue $\mathrm{kC}$, Kestin LL, Kini $\mathrm{V}$, et al. Impact of boost technique on outcome in carly-stage breast cancer patients treated with breastconserwing therapy. Am ] Clin Oncol 2001;24:26-32.

8. Habibollahi F, Mayles HM, Mayles WR et al. Assessment of skin dose and its rellacion to cosmesis in the conservative treatment of carly breast cancer. Int I Radiat Oncol Biol Phys 1988:14:291-6.

9. Hamers HP, Johansson KA, Venselaar JLM, De Brouwer P, Hansson U, Moudi C. Entrance and exit TL-dosimetry in the conservarive treatment of breast cancer: a pilot study for the EORTC Radiorherapy Cooperative Group. Radiother Oncol $199122: 280-4$.

10. Hammer J, Mazeron J-J, Van Limbergen E. Breast boost-why, how, when ...? Strahlenther Onkol 1999;175:478-83.

11. International Commission of Radiation Units and Measurements. Dose specificarion for reporting external beam therapy with photons and electrons. Report 50. Bethesda, Maryland USA: ICRU Publication; 1992.

12. McRae D, Rodgers J, Dritschilo A. Dose-volume and complication in interstitial implants for breast carcinoma. Int J Radiat Oncol Biol Phys 1987;13:525 -9.

13. Olivotio LA, Rose MA, Osteen RT, et al. Late cosmeric outcome after conservative surgery and radiotherapy: analysis of causes of cosmetic failure. Int J Radiat Oncol Biol Phys 1989;17:747-53.

14. Perez CA, Taylor ME, Halverson $K$, Garcia D, Kuske RR, Lackett MA. Brachytherapy or electron beam boost in conservation therapy of carcinoma of the breast: a nonrandomized comparison. Int \& Radiat Oncol Biol Phys 1996,34:995-1007.

15. Ptaszynski A-M, Van den Bogaert W, Horiot J-C, er al. Radration dose homogeneity in an EORTC multicenter trial on breast irtadiation. Acta Oncol 1999;38:19-27.

16. Resch $A_{*}$ Po"trer $R$, Van Limbergen E, er al. Long-tern results (10 years) of intensive breast conserwing therapy induding a high-dose and large-wolume interstitial brachytherapy boost (LDR/HDR) for T1/ T2 breasr cancer. Radiother Oncol 2002;63:47-58,

17. Romestaing $\mathbb{D}$, Lethingue $Y$, Carrie $C$, et all. Role of a $10-G y$ boost in the conservative treatment of early breast cancer: resul rs of a randomized clinical trial in Lyon, France. J Clin Oncol 1997:15:963-8.

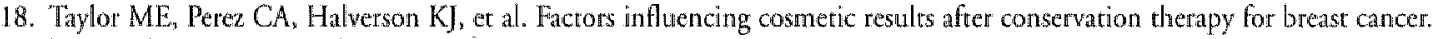
Int J Radiat Oncol Biol Phys 1995:31:753-64.

19. Van Dongen JA, Bartelink $\mathrm{H}$, Pentiman IS, et all. Long-term results of at randomized trial comparing breast-conserving therapy with mastectomy: European Organization for Research and Treatment of Cancer $1080 \mathrm{l}$ trial. I Natl Cancer Inst $2000 ; 92(14): 1143-50$.

20. Van Limbergen E, Van den Bogaert W, Van der Schueren E, Rijnders A. Timor excision and radiotherapy as primary treatment of breast cancer, analysis of patients and treatment parameters and local control. Radiother Oncol 1987;8:1-9.

21. Van Tienhoven G, Van Bree NAM, Miinheer BJ, Barrelink H. Quality assurance of the EORTC trial 22881/10882: "assessment of the role of the booster dose in breast-conserving therapy": the dummy run. Radiorther Oncol 1991:22:290-8.

22. Van Tienhoven G, Mijnheer B], Bartelink H, Gonzalez DG. Quality assurance of the EORTC Trial 22881/108:82: boost versus no boost in breast conserving therapy. An owerview. Srahlenther Onkol 1997;173:201-7.

23. Vicini FA. Horwitz EM, Lacena MD, at al. Long-term outcome with interstitial brachytherapy in the management of patients with earlystage breast cancer treated with breast-conserving therapy. Int I Radiat Oncol Biol Phys 1997;37:845-52.

24. Voogd AC, Nielsen M, Peterse IL, ce al. Differences in risk Gactors for local and distant recurrence after breast-conserving therapy or mastectomy for stage I and II breast cancer: pooled results of two large European randonized trials. I Clin Oncol $2001 ; 19(6): 1688-97$.

25. Vrieling $\mathrm{C}$, Colletre L. Fourquet A, et al. The influence of the boost in breast-conserving therapy on cosmeric ourcome in the EORTC 'boost versus no boose' trial. Inc J. Radiar Oncol. Bitol Ihys 1999;45:677-85.

26. Vrieling C, Colletre L, Fourquer A, ex all. The influence of patient, tumor, and treatrnent factors on the cosmetic results aster breastconserving therapy in the EORTC 'boost versus no boose' trial. Radionther Oncol 2000;55:219-32.

27. Wazer DE, Kamer B, Schmid C, Ruthazer R, Ulin K, Schmidt-Ullrich R. Factors determining outcorne in patients treated with interstitial implantation as a radiation boost for breast conservation therapy. Int J Radiat Oncol Biol Phys $1997 ; 39 \% 381-93$ 


\section{CHAPTER 5}

Quality Assurance in the EORTC phase III randomised 'boost vs. no boost' trial for breast conserving therapy: comparison of the results of two individual case reviews performed early and late during the accrual period.

Philip Poortmans, Fatma Ataman, J. Bernard Davis, Harry Bartelink, Jean-Claude Horiot, Marianne Pierart, Laurence Collette, Geertjan Van Tienhoven, On behalf of the EORTC Radiotherapy and Breast Cancer Groups.

Radiotherapy and Oncology accepted, 2005. 


\section{Abstract}

Background and purpose: To evaluate the impact of quality assurance on treatment compliance, we compared the outcome of the two individual case reviews (ICR) conducted early and late during the accrual period of a large prospective multi-centre trial.

Materials and Methods: At the onset of the trial medical files of 5 patients from each participating centre were evaluated for the compliance to the protocol for eligibility, surgery, pathology and radiotherapy and for the quality of reporting of the data on the case report forms. In 9 major centres, this procedure was repeated near the end of the trial.

Results: Both in the early and the late ICR we found a very limited number of deviations from the guidelines for eligibility, staging, surgery and pathology. Compliance to radiotherapy requirements was good with the exception of a too low minimal dose in $30 \%$ and the lack of target volume delineation in the majority of the evaluated patients. The comparison of the late with the early ICR demonstrated an improvement of the quality of data reporting by $6 \%$ and of target volume delineation from $33 \%$ to $53 \%$.

Conclusions: The initial ICR has lead to the identification of a number of parameters, which needed a clarification in the protocol. These items have been corrected and the individual institutions have been made aware of the necessary adaptations. The evaluation at the end of the trial period showed that there was an improvement but also showed that continuous monitoring is necessary, especially for institutions which have the most deviations in the first ICR.

Keyuords: Randomised clinical trial, Quality assurance, Individual case review, Breast cancer, Radiotherapy, Breast-conserving therapy. 


\section{Introduction}

A number of randomised trials and a meta-analysis have demonstrated similar survival rates in early breast cancer with breast conserving therapy (BCT) as with mastectomy $[5,6,20,23,29]$. However, radiotherapy (RT) schedules were not standardised and there are not enough data on the optimal total dose at the primary tumour site in BCT. The European Organisation for Research and Treatment of Cancer (EORTC) trial No. $22881 / 10882$ was designed to investigate the effect of the boost dose following whole breast radiation on local control, survival, and cosmetic outcome in stage I and II breast cancer patients treated with BCT. Patients with microscopically negative margins were randomised to receive an additional boost of $15 / 16$ Gy or no boost after 50 Gy of whole breast irradiation. The 5-year follow-up results of this trial showed a statistically significant decrease in the local recurrence rate with the additional boost dose from 7.3 to $4.3 \%$ [2]. A total of 251 patients with an incomplete excision was randomised between a boost dose of $10 \mathrm{~Gy}$ and $25 / 26 \mathrm{~Gy}$. Follow up is still too short to evaluate this part of the trial.

Based upon experience with institution-bound $[10,12,15,16]$ and trial-bound quality assurance (QA) programmes [27], an extensive QA programme was set up in the framework of this trial. This consisted in a dummy run procedure (DR) [26], an individual case review (ICR), in vivo dosimetry [8,9] and phantom dosimetry [26]. Before the start of the trial, a dummy run was organised to detect systematic errors and protocol ambiguities as early as possible. Thereafter an ICR, to detect residual ambiguities in the trial protocol and as well systematic and random errors, was conducted. This procedure was repeated at the end of the accrual period for the 9 major participating centres.

The results of this trial-bound QA programme are described elsewhere, with the exception of the ICR $[11,22,24,25]$. The current paper describes the analysis of the possible impact of QA on treatment compliance by comparing the outcome of the 2 ICR conducted early and late during the accrual period of the trial. Based on this, we can estimate the need for this type of trial-bound QA measures in future clinical trials.

\section{Materials and Methods}

From 1989 to 1996,32 institutions in 9 countries entered a total number of 5569 patients into EORTC trial 22881/10882. The trial required whole breast irradiation of $50 \mathrm{~Gy}$ in 5 weeks (2Gy/fraction/day) with two tangential opposing fields by Co-60 or by 4-8 MV photons. The boost dose had to be prescribed at the site of the primary tumour 
with a margin of $1,5 \mathrm{~cm}(3 \mathrm{~cm}$ for microscopically incomplete resected tumours and in case of an extensive intraductal tumour component). The technique of the boost could be electrons, photons or brachytherapy [18]. The dose to the whole breast should be specified at point A (generally the intersection of the beam axes). The boost dose had to be prescribed at point $B$, the centre of the primary tumour bed, irrespective of the dose delivered to this point with the whole breast RT [13]. The dose distribution in three planes had to be reported: the central plane (level of point $A$ ), tumour plane (level of point B), and the border plane at the other side of the tumour plane [3]. For treatment planning and evaluation, the whole breast target volume had to be delineated on the 3 transverse slices and point $B$ on the tumour plane.

The first ICR was carried our at the beginning of the accrual period, from October 1990 till October 1992. Twenty-one institutions, together responsible for $88 \%$ of the totall number of patients participated. The findings of this first evaluation were discussed immediately with the representatives of the institutions. Thereafter, a list of recommendations was distributed among all participants to clarify ambiguities in the protocol and case report forms, with the aim to improve compliance to the protocol. Based primarily on the findings of the DR, a revised version of the trial protocol was prepared and distributed. Towards the end of the accrual period, from October 1995 till October 1996, 9 major participants (together responsible for the accrual of $55 \%$ of the patients) participated in a second ICR. The findings of this second ICR were compared with the first one. In April 1997, 2 more centres, which started participating later, were reviewed for the first time.

For the ICR, the medical files of five parients were evaluated for compliance to the protocol guidelines and for the quality of reporting of patient-, tumour- and treatmentrelated parameters. To avoid the high costs and the time of on-site visits, participating institutions were invited to send a representative for an evaluation preceding one of the biannual meetings of the EORTC Radiotherapy Group. The participating centres received a list of 5 randomly selected parients only few days before the meeting. All but one of the parients selected for the second. ICR were treated after the first ICR of the same centre. The representative had to bring a complete set of medical information and RT data for each of those patients. The evaluation was performed by a QA review team consisting of radiation oncologists, radiation physicists and data managers. A review form containing numerous parameters on patient eligibility, surgery, pathology and radiotherapy (simulation, target volume delineation, dose specification, homogeneity, treatment execution, treatment verification) was used. When needed, the QA team delineated the target volume for the whole breast irradiation and point $\mathrm{B}$ to assess dose 
homogeneity. Treatment according to the other randomisation arm and a minimum dose $<90 \%$ or a maximum dose $>115 \%$ were defined as major protocol deviations. All other deviations were considered as being minor, including a minimum dose $90-94 \%$ and/or a maximum dose of $111-115 \%$ and the absence of delineation of target volumes. The number of major and minor protocol deviations was recorded per patient and per centre. The QA team reviewed an overall total of 159 patient files, including 10 patient files from 2 institutions, which started accruing patients later during the trial. They were evaluated for the first time in the second round of the ICR only and were therefore excluded from this analysis.

For all patients, the parameters on the trial case report forms (CRF) concerning eligibility, patient characteristics, staging, surgery, pathology, and radiotherapy were compared with the actual patient files for the transcription of information. We transferred the results of the evaluation to review forms, which were specifically designed for this purpose.

\section{Results}

\subsection{Compliance to the protocol (21 centres)}

In the first ICR, we reviewed a total number of 104 patients from 21 centres.

The vast majority of the patients was eligible (97\%). Staging (94\%), surgery (99\%) and pathology (99\%) were done according to the guidelines in the protocol for nearly all patients. Compliance to the RT requirements was excellent for dose specification, dose prescription, fractionation schedule and overall treatment time. A lower compliance, however, was observed in the documentation of the target volumes on the isodose plots and for dose homogeneity.

\subsection{Transcription of data from the medical files to the CRF ( 21 centres)}

Overall, the percent of correctly transcribed parameters was $82 \%$. The data were not available in the patient files, incorrectly transcribed, available in the file but missing on the CRF and reported on the CRF but missing in the file in $6 \%, 6 \%, 2 \%$ and $4 \%$ respectively.

\subsection{Comparison of first and second ICR (9 centres)}

Figure 1 displays the overall compliance figures for the early and the late ICR of the 9 major participating centres. In the analysis by institution, the compliance for eligibility, staging, surgery, and pathology was more than $90 \%$ for 8 centres. In one centre, 3 
patients had major deviations in the late ICR (all 3 were incorrectly staged of which one was also ineligible).

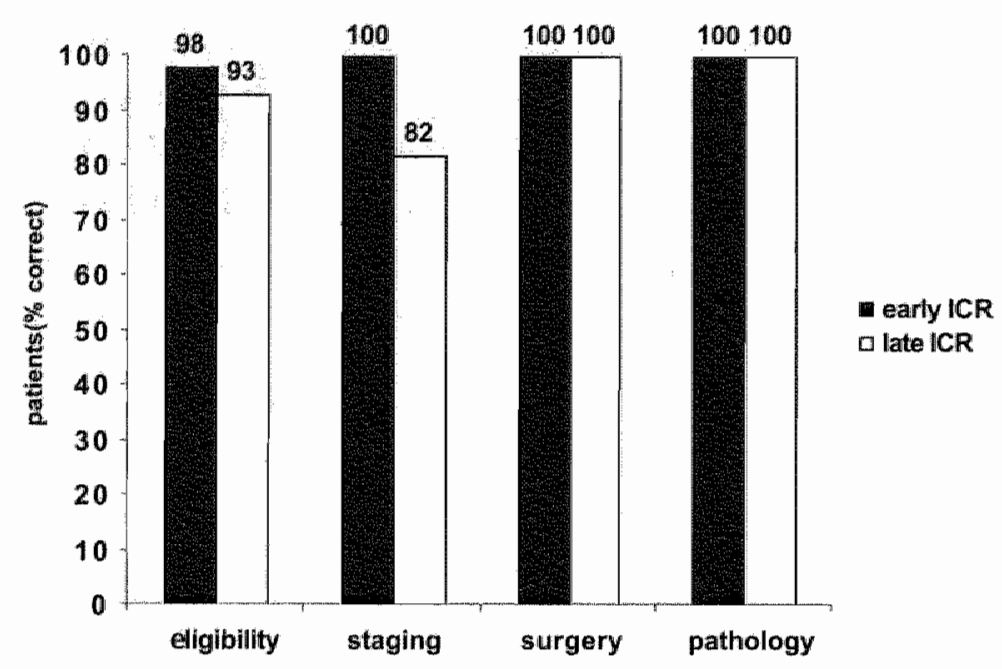

Figure 1: Comparison of the early and the late ICR for eligibility, staging, surgery and pathology (9 centres).

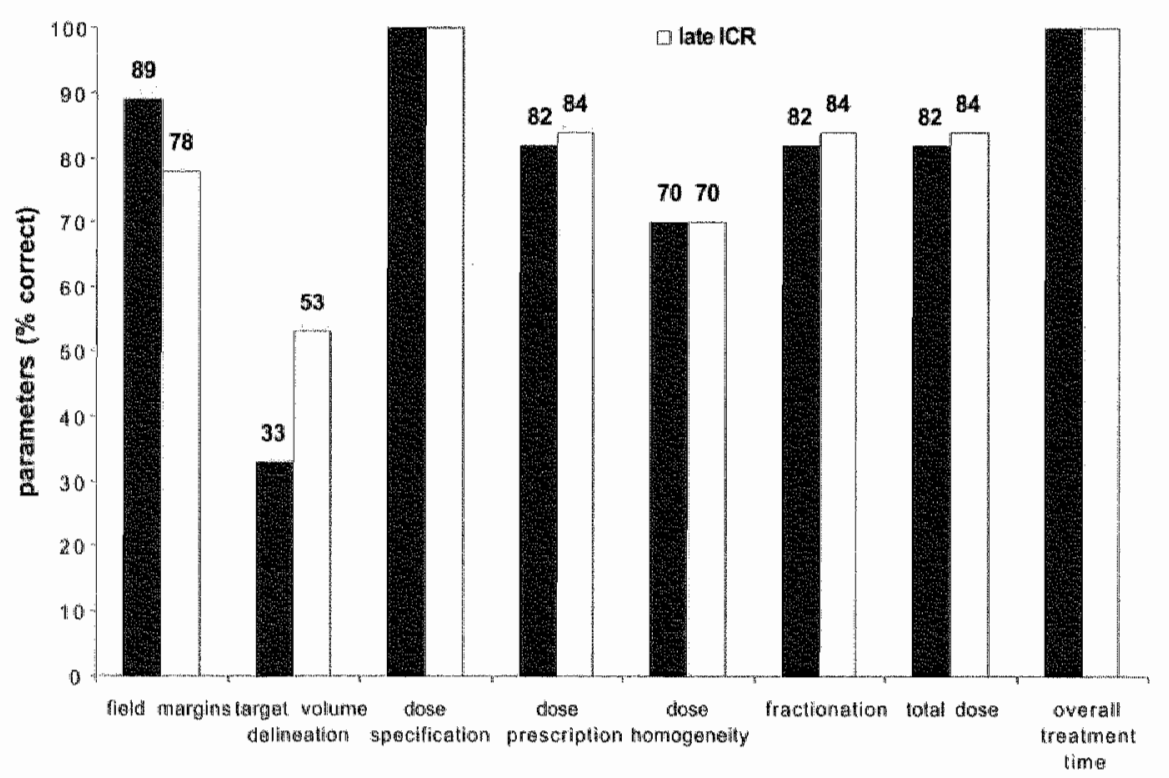

Figure 2: Comparion of the ealy and the late ICR for RT parameters (9 centres)

Compliance with RT requirements is shown in Figure 2. As the most important deviation we found that target volume delineation was missing on the treatment plans in $67 \%$ of the patients in the first and still $47 \%$ of the patients in the second ICR. Dose homogeneity was for $70 \%$ of the patients within the protocol requirements (range 47.5- 
$55 \mathrm{~Gy}$ ), equally for both ICR. In 11\% a major deviation (range 40-45 Gy) and in 19\% a minor deviation (range 45-47 Gy), always due to a too low minimum dose, was noted. The analysis by institution showed that the relatively low compliance rates with a number of RT requirements were caused by a few centres, which had deviations in all 5 randomly selected parients.

As displayed in Figure 3, the transfer of data from the patient files to the CRF was correct for most patients and most parameters, except for RT-related data. An overall improvement varying from 3 to $9 \%$ for data transcription from the first to the second ICR was noted. In the analysis by institution, there was an overall improvement in the quality of data transcription for 5 centres, of which 4 scored initially below $80 \%$. One centre had very poor data reporting ( $21 \%$ correct) in the early ICR, mainly due to missing information in $58 \%$ of the parameters. This centre improved its data reporting strikingly (over $30-80 \%$ for different parameters to $68 \%$ overall) in the late ICR. There were 3 centres with an overall low rate of approximately $65 \%$ correctly transcribed data on RT parameters. This improved in one of these centres, but further decreased in another centre.

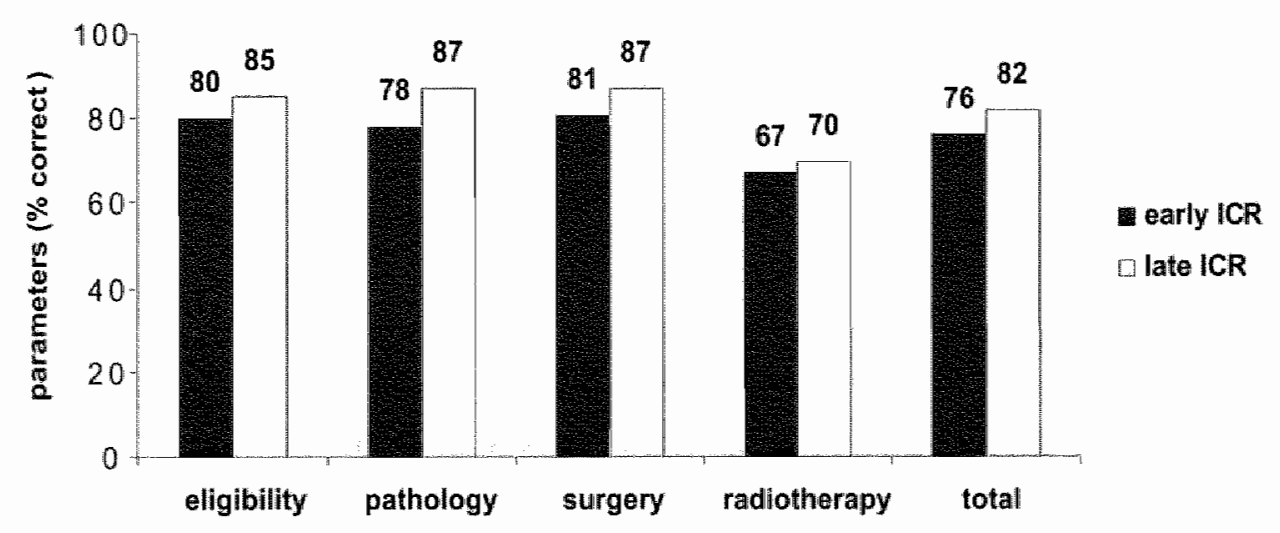

Figure 3: Comparison of the early and the late ICR for data transcription (9 centres)

\section{Discussion}

EORTC trial 22881/10882 demonstrated a significantly improved local control rate with an acceptable cosmetic outcome in early-stage breast cancer patients treated with breast conserving surgery and tangential field irradiation followed by a boost of $16 \mathrm{~Gy}$ to the primary tumour bed $[5,30]$. Especially for a large multi-institutional study with a long accrual period, it is of tremendous importance to monitor the quality of the treatment procedures all along the trial. In this paper, we summarised and compared the analysis of 
the two individual case reviews to evaluate the impact of quality assurance on treatment compliance and to estimate the need for trial-bound measures in future clinical trials. $U_{p}$ to our knowledge, a comparison between QA procedures performed early and late during the course of a clinical trial has not been reported before.

Only 21 of the 32 participating institutes completed the first ICR. However, since the 21 participants are the ones with the highest accrual (88\%) and 2 more centres participated later, this sample is representative of the total number of patients. The QA procedures were performed in the very early phase of the trial, before a significant number of patients was accrued. This enabled the study co-ordinators and the QA committee to give recommendations for improvements to the participants individually as well as during the meetings of the EORTC Radiotherapy Group. By doing so, participating institutes including those which had not yet participated to the QA programme or did not actually start patient accrual, were encouraged to improve the compliance to the trial protocol. In the second ICR, done at a later stage of the trial, a significant sample of 9 major participants, together accruing 55\% of the patients, was reviewed again. Ideally, participation to QA in the framework of clinical trials should be made obligatory. This is currently being introduced in some of the more recent and complex trials of the EORTC Radiotherapy Group.

\subsection{Compliance to the protocol (21 centres)}

A total of 38 parameters was reviewed for eligibility, staging, surgery and pathology. According to the guidelines of the protocol, only minor deviations and a limited interinstitutional variation were noted. Most aspects of the radiotherapy planning, prescription and execution were consistent with the protocol guidelines in the majority ( $\geq 80 \%$ ) of the evaluated patients. Treatment planning for tangential irradiation had to be done at 3 different transverse CT slices or manual outlines with delineation of the target volume. This was not routine practice at the start of this trial, when most centres defined field borders based on the patients' anatomy during the simulation procedure. This can explain the deviations for dose homogeneity which were always due to a too low dose at the borders of the target volume (drawn by the QA team when missing), most often in the off-axis treatment plans. Because we found nearly no breast cancer recurrences at the field borders, we judge this deviation however to be of less importance. The median dose and dose range within the target volume is similar to other prospective and retrospective studies $[1,7,14,21,28]$. Dose heterogeneity within the breast target volume with standard tangential fields is well documented, illustrating that with wedges only it is often not possible to comply fully with ICRU guidelines for dose homogeneity $[4,17]$. Today, target volume definition and dose homogeneity can be handled better with 
nowadays treatment facilities including IMRT. Remarkably, in half of the cases the target volume was not delineated, which might hamper proper treatment prescription and evaluation. This shortcoming has also been noted in a more recent EORTC trial [19]. Therefore, when writing future protocols, more attention should be paid to the proper definition and delineation of target volumes. The simplest way might be to recommend a strict adherence to ICRU guidelines, although discrepancies will always occur because of the different interpretation of the clinical data by different clinicians. Moreover, even today firm definitions for target volume delineation, for the whole breast as well as for the boost volume, are lacking. This can also explain the reluctance of the participants to delineate these target volumes.

\subsection{Transcription of data from the medical files to the CRF (21 centres)}

A good quality of data on the CRF is essential to relliably analyse the results of the trial. In only 3 of the 21 centres the rate of correct reporting of parameters on patient characteristics, eligibility, surgery and pathology was less than $80 \%$. However, about $10-$ $20 \%$ more errors and missing data were found for RT parameters. This can be explained by the more extensive information for RT details where nearly 3 times more parameters were evaluated. This very detailed verification of a large number of parameters turned out to be a heavy burden for the QA team, especially because of the large number of participants, the lack of financial support typical for academic trials and the need for a quick analysis and reporting to the participants.

\subsection{Comparison of first and second ICR (9 centres)}

Compliance to the protocol guidelines for eligibility, surgery, pathology and radiotherapy was high in both ICR. Dose homogeneity remained unchanged from the early to the late ICR for these nine centres. This can be explained because the treatment techniques remained largely unchanged during the course of this trial. Target volume delineation on isodose plots increased from 33 to $53 \%$, which was still lower than expected. The average percent of parameters correctly transcribed increased by $6 \%$ from $76 \%$ to $82 \%$ overall.

The overall good performance throughout the trial can be explained by a number of factors like the carefully written protocol, the instructions given at the start of the trial, the dummy run, the experienced investigators and the communication between study coordinators, QA team and investigators. The format of this kind of ICR proved to be adequate to evaluate the compliance to a clinical trial. Therefore, the costs and time of on site visits can be avoided in the framework of a multi-centre group like in the EORTC, probably unless severe deviations are found in the ICR or in another step of the 
QA programme. The review form that was prepared for this ICR was later developed into the "form 100 ", which is currently still the basis for the quality assurance review forms used for EORTC Radiotherapy Group trials (19).

\section{Conclusions}

In the individual case review a high compliance to the patient inclusion criteria was found. A number of potentially systematic deviations from the protocol or from general guidelines; were nevertheless detected. This may indicate that, even in protocols where, for reasons of accrual, the radiotherapy guidelines are kept as simple as possible, a more precise prescription of the radiotherapy procedures may be necessary. The high rate of mistakes in the transfer of RT data from the patient file to the CRF urges for RT specific training of data managers.

The initial ICR has lead to the identification of a number of parameters, which led to clarifications and corrections. The second ICR, done at a later stage of the trial, confirmed an improvement in the compliance to the guidelines of the protocol and in the transcription of data from patient files to CRF, which strengthens the reliability of the final trial results. This work thereby underlines the importance of a thorough QA programme. Financial and logistic support for QA in the framework of non-sponsored trials should be organised on a structural basis.

\section{Acknowledgements}

This publication was supported by grants number 3U10 CA1 1488-18SI through 5U10 CA11488-34 from the National Cancer Institute (Bethesda, Maryland, USA). Its content is solely the responsibility of the authors and does not necessarilly represent the official views of the National Cancer Institute. The Emmanuel Van der Schueren fellowship (Dr. Fatma Ataman) for Quality Assurance in radiotherapy is financially supported by the Vlaamse Kankerliga. The data management for the Dutch patients was supported by a grant from the Dutch Cancer Society.

This study was made possible by the co-operation of the following radiotherapy departments: Belgium: Brussels (Storme GA); La Louviere (Renaud A); Leuven (van den Bogaert W); Namur (Salamon E). France: Creteil (Calitchi GHM); Dijon (Horiot IC); Grenoble (Bolla M); Montpellier (Dubois JB); Paris (Fourquet A). Germany: Cologne (Müller RP); Dusseldorf (Roth S); Krefeld (Schulz U). Israel: Haifa (Kuten A). Switzerland: Geneva (Kurtz J); Lausanne (Mirimanoff RO). The Netherlands: 


\section{Amsterdam (Bartelink H); Heerlen (Jager JJ), Leiden (Leer JWH); Nijmegen (Hoogenraad W); Rotterdam (Koper PCM); Tilburg (Poortmans P); Utrecht (Struikmans H). United Kingdom: Nottingham (Morgan D).}

\section{References}

1. Arriagada $R, L e M G$, Rochard $F$, Contesso $G$. Conservative treatment versus mastectomy in carly breast cancer: patterns of failure with 15 years of follow-up data. linstitur Gustawe Roussy Brease Cancer Group. I Clin Oncol $1996 ; 14: 1558-1564$.

2. Bartelink $H$, Horiot $\mathbb{J C}$, Poortmans $\mathrm{P}$, et al. Recurrence rates after treament of breast cancer with standard radiocherapy with or without addirional radiation. N Engl J Med 2001;345:1378-1387.

3. Bartelink H, Horior JC, van der Schueren E. Phase III study in the conservative management of breast carcinona by tumorectomy and radiotherapy: assessment of the role of a booster dose of radiotherapy. Protocol $22881 / 10882$. EORTC Radiotherapy Cooperative Group EORTC Data Center, Brussels, Belgium.

4. Bucholtz TA, Gurgose E, Bice $\mathbb{E S}$, et al. Dosimerric analysis of intact breast irradiation in off-axis planes. Tnt J Radiat Oncol Biol Phys 1997;39:261-267.

5. Early Breast Cancer 'Trialists' Collaborative Group. Effects of radiotherapy and surgery in early breast cancer: an overview of randomised trials. N Engl Med 1995;333:1444-1455. [Erratum, N Engl J Med 1996;334:10031.

6. Fisher B, Anderson S, Redmond $\mathrm{CK}_{n}$ Wolmark N, Wickerham DL, Cronin WM. Reanalysis and results after 12 years of follow-up in a randomised clinical rrial comparing total mastectomy with lumpectomy with or wirhout irradiation in the trearment of breast cancer. N Engl J Med 1995;333:1456-1461.

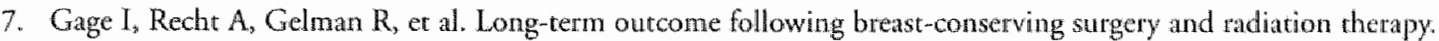
Inc J Radiat Oncol Biol Phys 1995,33:245-251.

8. Hamers HP, Johansson KA, Venselaar \L, de Brouwer P, Hansson U, Moudi C. Entrance and exit TL-dosimerry in the conservative treatment of breast cancer: a pilot study for the EORTC-Radiotherapy Cooperarive Group. Radiother Oncol. 1991;22:280-284.

9. Hamers HP, Johansson KA, Venseliar JL, de Brouwer P, Hansson U, Moudi C. In vivo dosimetry with THD in conservative treatment of breast cancer patients treated with the EORTC protocol 22881. Acta Oncoll $1993 ; 32: 435-443$.

10. Hansson U, Johansson KA. Qualiry audit of radiotherapy with EORTC mailed in warer TL-dosimerry, Radiother Oncol, 1991;20:191-196.

11. Heukelom $S_{x}$ Lanson $J H$, wan Tienhoven $\mathrm{G}$, Mijnheer B]. In vivo dosimetry during angential breast treatment. Radiorher Oncol 1991:22:269-79.

12. Horiot JC, Johansson KA, Gonzalez DG, van der Schueren E, van den Bogaert W, Notter G. Quality assurance control in the EORTC cooperative group of radiotherapy. 1. Assessment of radiotherapy staff and equipment. Radiorher Oncol 1986;6:274-284.

13. ICRU Report 50. Prescribing, Recording and Reporting Phoron Beam therapy. International Commission on radiation Unit and Measurements. 7910 Woodmonc Avenue, Bethseda, MD 20814, USA.

14. Jacobson JA, Danforth DN, Cowan KH, er al. Ten-year restults of a comparison of conservarion wirh mastectomy in the trearment of stage I and II breast cancer. N Engl J Med 1995;332:907-911.

15. Johansson KA, Horiot JC, Van Dam J, Lepinoy D, Sentenac I, Sernbo G. Quality assurance control 2. Dosimetric intercomparison. Radiother Oncol 1986;7:269-279,

16. Johansson KA, Horiot JC, van der Schueren E. Quality assutance control in the EORTC cooperarive group of radiotherapy. 3. Intercomparison in an anatomical phanton. Radiother Oncol, 1987;9:289-298.

17. Krasin M, McCall A, King S, et al. Evaluation of Standard Breast Irradiarion Technique: A Dose-Volume Analsis of Tangential Irradiation using Three Dimensional Tools. Int J Radiat Oncol Biol Phys 2000;47:327-333.

18. Poortmans $\mathrm{P}$, Bartelink $\mathrm{H}$, Horior $\mathrm{JC}$, ct al. The influence of the boost technique on local control in breast conserving treatment in the EORTC "boost versus no boost" randomised trial. Radiother Oncol 2004:72:25-33.

19. Poortmans $P_{3}$ Kouloulias VE, Venselaar JL, et al. Qualiry assurance of EORTC trial 22922/10925 investigating the role of internal mammary - medial supraclavicular irradiation in stage L-III breast cancer: the individual case review. Eur ] Cancer 2003;39:2035-2042. 
20. Sarrain $\mathrm{D}$. Le $\mathrm{MG}$, Arriagada $\mathrm{R}$, et all. Ten-year results of a randomised trial comparing a conservative treatment to mastectomy in early brease cancer. Radiother Oncol $1989 ; 14: 177-184$.

21. Touboul $\mathrm{E}$, Buffat $\mathrm{L}$, Belkacumi $\mathrm{Y}$, et al. Local recurrences and distant metastases after breast-conserving surgery and radiation therapy for early breast cancer. Int J Radiat Oncol Biol Phys 1999;43:25-38.

22. Van Bree NA, van Batrum LJ, Huizenga $H$, Mijnheer BJ. Three-dimensional dose distribution of tangential breast treatment: a nationall dosimetry intercomparison. Radiother Oncol 1991;22:252-260.

23. Van Dongen JA, Voogd AC, Fentiman IS, et al. Long-term results of a randomised trial comparing breastconserving therapy with mastectomy: European Organization for Research and Treatment of Cancer 10801 trial. I Nat Cancer Inst 2000;92:1143-1150.

24. Vin Thenhoven $G$, Lanson JH, Crabeds D, Heukelom S, Mijnheer BI. Accuracy in tangential breast treatment setup: a portal imaging study. Radiother Oncol $1991 ; 22: 317-322$.

25. Van Tienhoven $G$, Mijnheer BJ, Bartelink H, Gonzalex DG. Qualicy assurance of the EORTC Trial 22881/10882: boose vertsus no boost in breast conserving therapy. An overview. Strahlenther Onkol, 1997;173:201-207.

26. Van Tienhowen G, Van Bree NAM, Mijnheer B and Bartelink H. Quality assurance of the EORTC mial 22881/10882: "assessment of the role of the booster dose in breast conserving therapy": the Dummy Run. FORTC Radiotherapy Cooperarive Group. Madiother Oncol, 1991:22:290-298.

27. Vantongelen K, Rotmens. N, van der Schueren E. Quality control of validity of datal collected in clinical trials. EORTC Study Group on Data Management (SGDM). Eur J Cancer Clin Oncol, 1989;25:1241-1247.

28. Veronesi $U, B a n f i$, Salvadori $B$, et al. Breast conservation is the treatment of choice in small breast cancer: longterm results of a randomized trial. Eur J Cancer 1990;26:668-670.

29. Veronesi $U_{y}$ Salvadori $B$, Luini $A$, et al. Breast conserwation is a safe method in patients with small cancer of the breasti long-term results of three randomised trials on 1973 patients. Eur J Cancer 1995;31:1574-1579.

30. Vrieling $C$, Collette $L$, Fotrquet $A_{F}$ Hoogenraad WJ, et al. The influence of the boost in breast-conserving therapy on cosmetic outcome in the EORTC "boost versus no boost" trial. EORTC Radiotherapy and Breast Cancer Cooperative Groups. European Organization for Research and Treatment of Cancer. Int J Radiar Oncol Biol Phys $1999 ; 45: 677-685$ 


\section{CHAPTER 6}

The potential impact of treatment variations on the results of radiotherapy of the internal mammary lymph node chain: a quality-assurance report on the dummy run of EORTC phase III randomized trial 22922/10925 in stage I-III breast cancer.

Philip M. P. Poortmans, Jack L. M. Venselaar, Henk Struikmans, Coen W. Hurkmans, J. Bernard Davis, Dominique Huyskens, Geertjan van Tienhoven, Vernon Vlaun, Jan J. W. Lagendijk, Ben J. Mijnheer, Karin A. De Winter, Marleen van der Hulst and Walter EC. van den Bogaert.

Int. J. Radiat. Oncol. Biol. Pbys. 49: 1399-1408, 2001. 


\section{Abstract}

Purpose: To present the results of the dummy run of the European Organization for Research and Treatment of Cancer (EORTC) trial investigating the role of adjuvant internal mammary and medial supraclavicular (IM-MS) irradiation in Stage I-III breast cancer.

Materials and Methods: All participating institutions were asked to produce a treatment plan without (Arm 1) and with (Arm 2) simultaneous IM-MS irradiation of 1 patient after mastectomy and of 1 patient after lumpectomy. Thirty-two dummy runs have been evaluated for compliance to protocol guidelines, with respect to treatment technique and dose prescription.

Results: A number of more or less important deviations in treatment setup and prescription have been found. The dose in the IM-MS region deviated significantly from the prescribed dose in 10\% of the cases for Arm 1, and in 21\% for Arm 2. Assuming a true 5\% 10-year survival benefit from optimal IM-MS irradiation, an increase of only $3.8 \%$ will be found due to this suboptimal dose distribution.

Conclusions: In the dummy run, a number of potential systematic protocol deviations that might lead to false-negative results were detected. By providing recommendations to the participating institutions, we expect to improve the interinstitutional consistency and to promote a high quality irradiation in all institutions participating in the trial.

Keywords: Quality assurance, Breast cancer, Radiotherapy, Randomized trial, Internal mammary node. 


\section{Introduction}

In operable breast cancer, the influence of regional lymph node involvement on prognosis is well known. The incidence of lymph node invasion of the internal mammary chain ranges from $4 \%$ in axillary node-negative patients with upper lateral tumor location to $72 \%$ in axillary node-positive patients with lower medial tumor location (1-7). Despite this significant risk of tumoral involvement and despite several decades of combination of surgery and radiotherapy in the local treatment of breast cancer, there remains an important controversy concerning the effectiveness of irradiating the regional lymph node areas. Although in a large number of studies a significant decrease in locoregional recurrence was found ( $8-16)$, a survival benefit could not be demonstrated for patients irradiated to the regional lymph node areas. Some reports have even shown a negative effect on long-term survival (17-20). One of the latest meta-analyses, however, demonstrated a reduction of long-term breast cancer death rate in the group of irradiated patients (18). This was only recently confirmed in three randomized trials, which demonstrated the positive impact of adjuvant locoregional irradiation on survival in high-risk patients (21-24). In these trials, it was not clear what resulted in the advantage: the prevention of local recurrences through irradiation of the chest wall, or the adjuvant regional irradiation treating micrometastases in lymph nodes, or both. To date, only one randomized radiotherapy trial on the effectiveness of adjuvant internal mammary irradiation alone is published (25). The follow-up is too short and the number of patients too limited (270 patients total) to provide an answer on the usefulness of adjuvant internal mammary irradiation.

A number of authors have pointed out statistical as well as technical flaws in a large number of these studies. They have questioned the validity of the conclusions, based on the treatment results of different treatment periods and different techniques $(4,26,27)$. It has been suggested that the lack of evidence of a survival benefit was largely due to insufficient numbers of patients treated in an accurate way (28) and that inadequate treatment techniques, resulting in too high doses to the heart, could explain the negative effect found in some studies $(17,18,29)$. This was confirmed by a report from the Stockholm group, which clearly demonstrated that too high doses to the myocardium would result in a higher death risk from ischemic heart disease (30).

In May 1996, the Radiotherapy and the Breast Cancer Groups of the European Organization for Research and Treatment of Cancer (EORTC) initiated a large Phase III randomized multicenter trial. Its objective was to investigate the value of adjuvant irracliation of the internal mammary and medial supraclavicular (IM-MS) lymph node chain in patients with localized breast cancer Stage I-III with medially or centrally 
located tumors and/or axillary lymph node invasion (31). The principal goal of this trial, in which patients are randomized between irradiation of the IM-MS lymph nodes or not, is to solve the controversy about the possible benefit of IM-MS irradiation on survival. Furthermore, the question of the importance of the possible side effects on the heart and the lungs will be examined in an era of modern radiotherapy techniques.

\begin{tabular}{ll}
\hline Patient position & Supine \\
\hline Field borders & Lateral: middle clavicle \\
& Upper: $3 \mathrm{~cm}$ above medial clavicular head \\
& Medial: $1 \mathrm{~cm}$ across midline \\
& Lower: lower border 4 th rib (lower in inferior located rumors) \\
\hline Dose prescriprion according & Photons: at $3 \mathrm{~cm}$ depth (max $10 \mathrm{MV}) ; 13 \times 2 \mathrm{~Gy}$ \\
to ICRU Reports 50 and 62 & Electrons: at depth of Dmax (12 to $14 \mathrm{MeV}) ; 12 \times 2 \mathrm{~Gy}$ \\
\hline
\end{tabular}

ICRU a International Commission on Radiation Unirs and Measurements

Table I: Requivemexts for standard treament technique and dose prescription

A large number of patients (3780) and a long follow-up (10 years) will be needed to answer the questions of this trial. To allow many radiotherapy institutions to participate in the trial and accrue a large and representative sample of patients, a simple so-called standard treatment setup technique for the IM-MS irradiation is recommended and described in the protocol (Table 1). In this technique, the IM-MS lymph node area is treated with mixed photon and electron beams matched to the tangential field borders of the breast or thoracic wall (which can alternatively be treated with a direct electron field). Exact localization of the internal mammary nodes is not mandatory for the standard technique but the use of a CT scan; ultrasound or lymphoscintigraphy is recommended to localize the IM-MS lymph nodes in the patient. This information can be used to select the electron beam energy, the prescription depth for the photon beam and the relative beam weights. Several inscitutions have developed specific irradiation techniques for this indication. More complex treatment setups may be accepted in the trial, provided that these are in accordance with the basic recommendations. If the use of an alternative technique is deemed necessary, then individual localization of the internal mammary nodes becomes mandatory. The optimal beam dimensions depend on the location of the nodes and the method of measurement.

The quality assurance (QA) program of the EORTC Radiotherapy Group aims at testing the ability of the participating institutions to cope with protocol guidelines. The 
variations in the dose delivered due to uncertainties in treatment-planning systems (TPS) and in the calibration of the treatment machines are investigated by the general EORTC Radiotherapy Group QA procedures for institutional infrastructure. In the early phase of clinical trials various QA procedures are performed to check protocol compliance and to detect and correct possible ambiguities in the protocol, which might introduce systematic protocol deviations $(28,32-36)$. In EORTC trial 22922/10925 the specific QA program consists of (1) control of data consistency at the EORTC Data Center by a double data entry procedure in the database, (2) a dummy run procedure, (3) an early eligibility and treatment compliance check, and (4) mailed TLD electron dosimetry.

The current paper describes the outcome of the analysis of the dummy run from 32 major participating institutions. The potential influence of inevitable variations between institutions or individual patients in treatment setup and dose to the IM-MS nodes on the final outcome of the trial is estimated. Possible implications are discussed.

\section{Materials and Methods}

A set of 3 CT-based life-size transverse slices together with all information necessary for treatment planning of 1 anonymous and representative patient after mastectomy on the left side (Fig. 1) and for 1 patient after a lumpectomy in the right breast (Fig. 2) was sent to all radiotherapy departments that had expressed their intention to participate in the trial. The participants were asked to develop a treatment plan based on their radiotherapy equipment and to calculate a complete set of isodose distributions with their TPS, according to each arm of the protocol for a clinical target volume (CTV) $(37,38)$ that was limited to the thoracic wall/breast only and for a CTV that included the thoracic wall/breast as well as the IM-MS lymph node region. If an institution used different techniques for treating such patients, the dummy run procedure was repeated for each of these techniques. If treatment technique was changed during participation in the trial, the dummy run had to be repeated for the new technique. The dummy run was not intended for irradiation of the IM-MS alone for cases in which, according to the local treatment guidelines, irradiation of the thoracic wall was not indicated.

Data from the participating institutions were mailed to Tilburg where they were checked within weeks of receipt by the coordinating radiation oncologist and physicist. 'This allowed a quick detection of possible protocol deviations and enabled a prompt response to the participaring institutions in the form of a letter. The following points were discussed in this letter: treatment technique; extent of irradiation of normal tissues; dose homogeneity in the CTV; dose prescription; and a general conclusion. In most cases, 

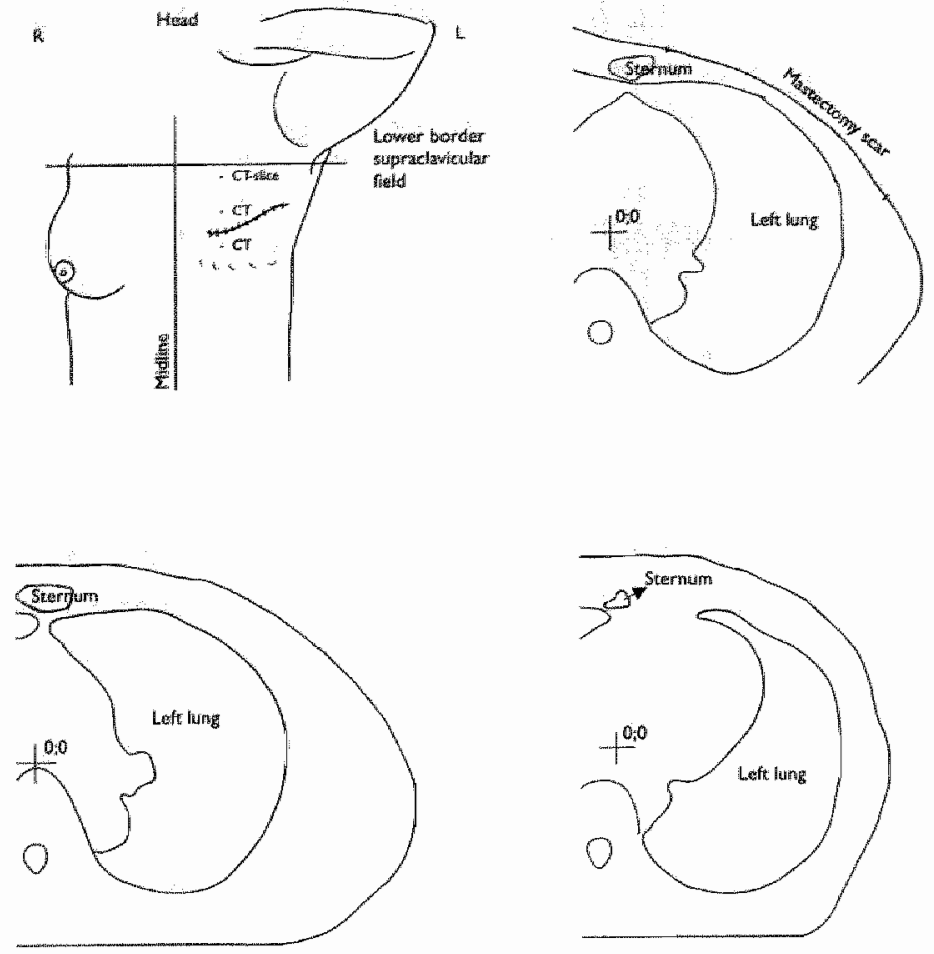

Figure 1: Pavient 1 after mastectomy of the left side.
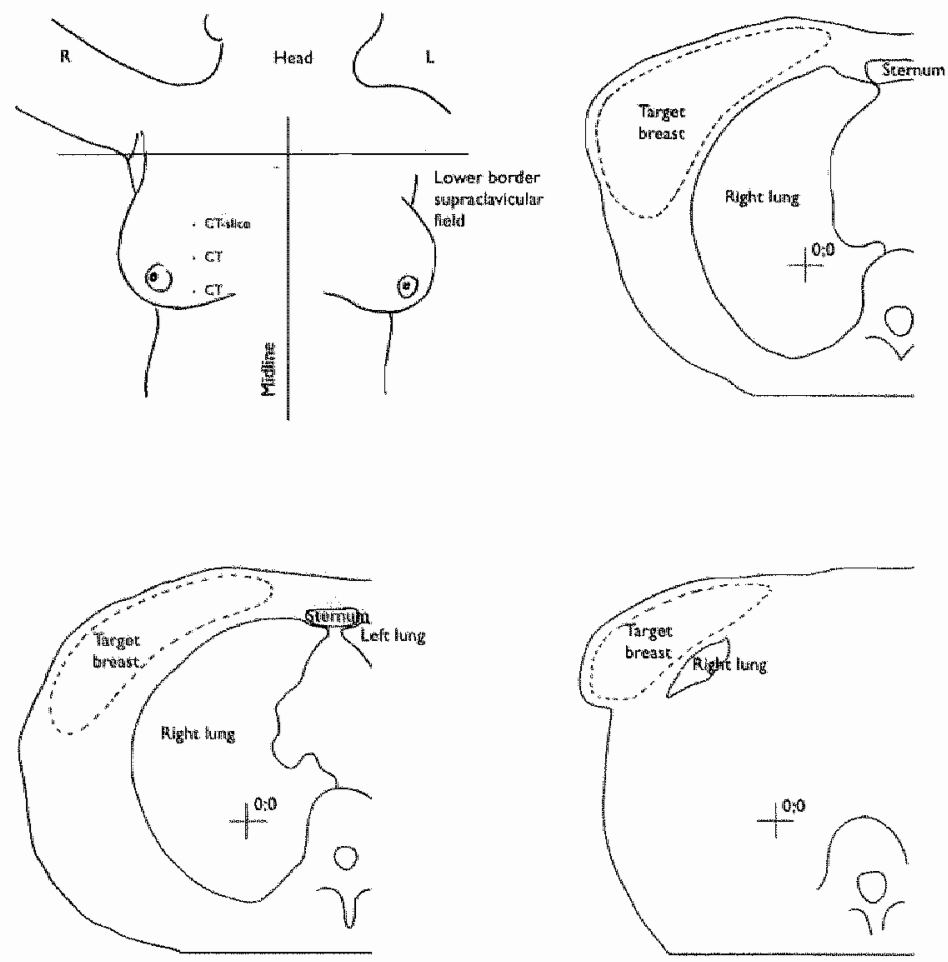

Figure 2: Patien 2 after lumpectomy of the right breast. 
participants were asked to clarify or to complete the information supplied. Further evaluation of the data consisted of a quantitative assessment of the compatibility of the treatment techniques with the protocol. At regular time intervals, a QA committee meeting was organized to discuss the findings and to complete the provisional answer already sent to the participating institutions. These findings were discussed again with the institutions on an individual basis.

Based on the dummy run, a letter with recommendations to improve protocol compliance was sent to all participants. At every biannual meeting of the EORTC Radiotherapy Group an updated overview of the findings was presented to keep participants well informed on possible ambiguities in the protocol prescriptions.

Because irradiation of the medial supraclavicular part of the IM-MS lymph node region is very straightforward, the dummy run focused on the irradiation of the internal mammary part of the IM-MS lymph node region. To avoid possible misunderstandings, this will be referred to as IM-MS from here on.

The location of the IM-MS lymph nodes varies considerably, both in the lateral and in the ventrodorsal direction. Approximately $85 \%$ of the lymph nodes lie within $4 \mathrm{~cm}$ of the midline laterally and at a depth of $4 \mathrm{~cm}$ or less in the first three intercostal spaces (39, 40). Because of the closeness of the IM-MS lymph nodes to the skin of the patient, a supplementary margin of only $0.5 \mathrm{~cm}$ has to be added to this CTV to obtain the planning target volume (PTV) $(37,38)$. To cover this with at least the $95 \%$ isodose line for photons and $85 \%$ for electrons, the prescription point for photons is defined at $3-\mathrm{cm}$ depth, and the electron energy has to be between 12 and $14 \mathrm{MeV}$, unless individual localization of the IM-MS is performed. For the evaluation of the calculated dose at the IM-MS, two reference points were defined for each slice: the point $\mathrm{P}(\mathrm{IM})$ represents an estimation of the anatomic location of the IM lymph nodes; the point $\mathrm{P}(3,3)$ is the point located $3-\mathrm{cm}$ below the skin and 3-cm homolateral of the midline of the patient (Fig. 3).

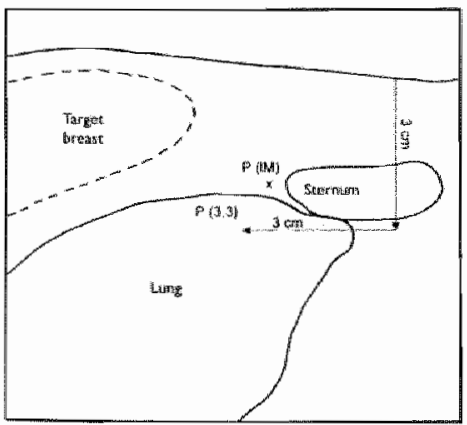

Figure 3: Illustration of the definition of points $P(M M)$ and $P(3.3)$. 
These points were not previously outlined on the CT-based slices sent to the participants. The calculated dose at both points was measured on the central slice and on the cranial slice for the dummy run treatment plans according to both arms of the protocol, i.e., without and with IM-MS irradiation. Because tumoral involvement of the IM-MS lymph node region occurs mostly in the first three intercostal spaces, the calculated IMMS dose at the caudal slice was deemed not relevant.

The statistical considerations of the trial are based on an expected survival rate in the control group withour IM-MS irradiation of $50 \%$ at 10 years, and a minimal potential benefit of clinical significance of $5 \%$ to $55 \%$ survival at 10 years in the patient group with IM-MS irradiation (31). To estimate the theoretical influence of inter-institutional dose variation to the IM-MS lymph node chain on the outcome of the trial, we assumed, based on the typical sigmoidal cell survival curves, that a dose of $0-49 \%$ to the IM-MS region has no effect, a dose of $50-84 \%$ has $10 \%$ of the effect and a dose of higher than $85 \%$ has the full effect on survival.

\section{Results}

By July 1996, the first patients were entered in the study. By February 2000, 39 institutions in 12 different countries had expressed the wish to participate in the study. At that time, 5 of them had not included any patient in the protocol. The dummy run procedure was completed by 32 institurions (Table 2 ).

Irradiation of the breast alone was always performed by two tangential fields, while the irradiation of the thoracic wall after mastectomy was done in 18 institutions by 2 tangential photon fields, in 9 institutions by a direct electron field; in 3 institutions, no data were given on irradiation of the thoracic wall after mastectomy, and, in 2 institutions, the technique was individualized. For the combined irradiation of the IMMS lymph node chain and the breast/thoracic wall, 25 institutions used the standard technique as described in the protocol, and 7 institutions presented an alternative technique. Several participating institutions presented more than one treatment technique for combined irradiation of the IM-MS and the breast/thoracic wall target volumes. One institution stated that they would only include patients after lumpectomy, as only very few patients are referred to them after mastectomy. Another institution only participates with postmastectomy patients who do not have an indication for irradiation of other target volumes to avoid the problems of beam matching at the junction of the IM-MS field with the breast/thoracic wall fields. 


\begin{tabular}{|c|c|c|c|}
\hline City & Country & Institutilion: & $\begin{array}{l}\text { Local responsible radianon oncologust } \\
\text { and physicist }\end{array}$ \\
\hline Bruselles & Belgiuns. & University Hospiral Sam-Luc & C. Kirkliove \\
\hline Lewven & Belginm & Universiry Hospiral & Wwan den Bogaer, D.Huyskns \\
\hline Wilrijk & Belgrum & AZ Sin-atuguscinus: & Ph. Huger, K. Haest \\
\hline Sanriago & Chile & Instituto de Radiomedicina & R.Aritagadat, L.Shwartzman \\
\hline Besancon & France & University Hospital & V.Magnin, ].FBosset \\
\hline Bordeaux & France & Institut Bergoniế & 1. M Dilhuydy, S Landrian \\
\hline Dijon & France & Centre G.-FLederc & I.Barillor, S. Naudy \\
\hline Grenoble & France & Unversicy Hospital & M.Bolla, A. Dusserre \\
\hline Nice & France & Centre Artoine-Lacassagne & J Lagrange, Mirelse \\
\hline Rennes & France & Cantre Regional Eugene Marquis & C.Chenal \\
\hline Villejuif & France & Institut Gustave Rroussy & J.Ph. Pignol, H. Boullnilk \\
\hline Berlin & Germany & Universicy Hospital Charitể & VBudach, J.Groll, U.Jahn \\
\hline Götringeda & Germany & University Hospital & wain Drünen \\
\hline Köln & Germany & University Hospital & R.Bongartz \\
\hline Tübingen & Gemany & University Hospiral & N.Weidner \\
\hline Harfa & Israel & Rambam Medical Center & Elahu Gez, R. Bar Deroma \\
\hline Tel-Hashomer & Israel & Chaim Sheba Medical Center & M. Raphal Pfefter, J.Gotfman \\
\hline Como & Iraly & Ospedale Sant'Anna. & M. Walli, A.Monti, A.Ostinellí \\
\hline Genova & Italy & Isc. Naz. per la riserca sill cancro & EFoppiano, MGuenzi \\
\hline Gidansk & Poland & University Hospital & D. Jassem \\
\hline Barcelona & Spain & Instituto Català d'Oncologga & S. Villia, EMoreno \\
\hline Genère & Switzerland & Uniwersity Hospitall & J. Kurtz, Ph. Nouer \\
\hline Lausanne: & Switzerland & University Hospiall & W. Jeanneret, H. Do \\
\hline Zürich & Swikgerland & University Hospiral & Ch,Glanzmann, J. BS. Davis \\
\hline Amsterdan & The Nerherlands & Nederlands Kanker Instiruut & B. Pierers, C. Hurkmans \\
\hline Deventer & The Nerherlands & RTI Sted endriehoek e: 0 . & A.Mak, A.wan st Rier \\
\hline Enschede & The Nerherlands & Medisch Spectrum Twente & J.J Jobsen \\
\hline Groningen & The Netherlands & University Hospital & W. Dolsma, Revan dex Hullst \\
\hline Tilburg & The Nerhertlands & Dr. Bernard Verbeten Instiituut & Ph. Pootrinatus J Wenselate \\
\hline Utrecht & The Netharlands & University Medical Center & H. Struikmanss I. I.agendijk \\
\hline Istanbus! & Tukey & University Hospital & Mincer \\
\hline Norringham & Unitad Kingdon & Notringhatm City Hospital & D.Momgan, J Berridge \\
\hline
\end{tabular}

Table 2: List of participants in the dummy run.

A large number of minor and major deviations from the protocol or from general guidelines were encountered through the dummy run procedure, most of them possibly leading to potential systematic protocol deviations (Table 3). The comments to the deviations can be divided into general remarks, specific remarks on the irradiation of the IM-MS, and remarks related to the irradiation of the other target volumes. No institution was excluded from the trial because of unacceptable protocol deviations, although, in 2 institutions, modifications were made to the technique. This resulted in a repeat of the dummy run for those institutions. 


\subsection{General remarks}

In 7 cases, relewant treatment-related data were incomplete. Also, 7 institurions did not provide a treatment plan for irradiation of the breast/thoracic wall alone (Arm 1). Another frequently occurring protocol deviation was that the dose prescription and normalization were not according to the protocol or to the ICRU guidelines $(37,38)$. Some of the most common general deviations in fact only hampered the evaluation of the durnmy run itself: in the majority of the treatment plans too few isodose lines (e.g., $70,90,110,130 \%$ ) were plotted, and, in two dummy runs, the internal structures were not drawn on the treatment plans, or the magnification factor of the plan was not given or even different from slice to slice.

\subsection{Remarks on the irradiation of the IM-MS clinical target volume}

The length of the IM-MS field often varied, without an explanation for the relation of the length of this field to the relative position of the primary tumor. If the IM-MS field was too short, in some occasions the lower medial part of the breast/thoracic wall was not covered by any of the treatment fields. Areas of overdose or underdose were present at the junction zone between the IM-MS lymph node region and the breast/thoracic wall. This over- or underdose depended on the presence or absence of an overlap with the breast/thoracic wall fields as well as on the position of the IM-MS field itself. In 11 cases, the IM-MS electron beam energy was not according to the protocol prescription (12-14 $\mathrm{MeV})$. In 8 cases, the dose prescription of the IM-MS photons was not at the required $3-\mathrm{cm}$ depth, and, in 6 cases, the prescribed photon and electron dose was not $26 \mathrm{~Gy}$ and $24 \mathrm{~Gy}$, respectively, without specifying that individual localization of the IM-MS lymph nodes had been done.

\section{General remarks:}

- Too few isodose lines

- Dose prescription not acoording to ICRU recommendations

- Normalization not on whole plan

- Missing beam data / monitor units calloulations

- No plans previded for arm 1 (without IM-MS)

Dose prescription not $25 \times 2$ Gy

- No lung densiry correction

- Electron fields not planned

- No drawing of internal structures

- Magnificarion fincor different from plan ro plan

- Only inclusion of specific patient subgroups

- Separate elecron and phoron plans 
Specific remarks on the irradiation of the IM-MS lymph node chain

- Lengch IM-MS, too long or woo shorr

- Overlap IM-MS and breast thoracic wall fields, cave hot spot 14

- Exact natch ar juncrion, cave underdose.

- MM-MS elactron energy II

- Dose prescription IM-MS photons 8

- Position IM-MS field, cave underdose 6

Proportion photons/electrons not $26 \mathrm{~Gy} / 24 \mathrm{~Gy}$

Remarks on the irradiation of the breast/thoracic wall:

- Too low minimal dose to breast/thomacic wall CTV 18

- Dose to IM-MS (unintended) $\geq 25 \%$

- Tangential fields ar $180^{\circ}$

- Wide fields around targer volume

Ton large or too low wedge faction 2

Table 3: Remarles on the dummy mn.

\subsection{Remarks on the irradiation of the other target volumes (breast/thoracic wall)}

In 18 cases the calculated dose to the breast/thoracic wall CTV was too low, generally due to too narrow margins around the CTV. In $11 / 24$ dummy run treatment plans without IM-MS irradiation, the dose to the IM-MS lymph node region was considered too high $(\geq 25 \%)$. Other less frequent deviations were mainly concerned with treatment plans using a less than optimal beam set-up.

\subsection{Dose to the IM-MS region in both randomization arms}

The calculated doses at the points $\mathrm{P}$ (IM) and $\mathrm{P}(3,3)$ as determined on the central slice and the cranial slice are displayed in Table 4 for the treatment plans "without IM-MS irradiation" (Arm 1) and in Table 5 for the treatment plans "with IM-MS irradiation" (Arm 2). The determined dose values were categorized as: $<25 \%, 25-49 \%, 50-74 \%$, $75-84 \%, 85-94 \%$ and $\geq 95 \%$ of the prescribed dose ( $50 \mathrm{~Gy}$ ). The relative distribution of the number of treatment plans in the dose categories is further summarized in the categories $<25 \%, 25-84 \%$, and $\geq 85 \%$. In Table 4 , results are shown for the plans with tangential beam techniques and the plans with electron field techniques (used in 1/3 of the mastectomy patients) separately. In the latter category, a higher percentage of plans showed an unintended high dose to the points $\mathrm{P}(\mathrm{IM})$ and $\mathrm{P}(3,3)$. Table 5 illustrates that the calculated dose to these points is more variable among the institutions that used a standard technique (used in 78\%) as compared to those that used alternative techniques (used in $22 \%$ ) with less underdose to the $\mathrm{P}$ (IM) and $\mathrm{P}(3,3)$ points for the alternative techniques. 


\begin{tabular}{|c|c|c|c|c|c|c|}
\hline \multicolumn{7}{|c|}{ TANGENTLA FUEDSTO WREAT/THORACIC WALL } \\
\hline Tof pursecribed dose: & $<25 \%$ & $25-49 \%$ & $50-760 \%$ & $75-84 \%$ & $85-94 \%$ & $\geq 95 \%$ \\
\hline PIIM $\mathrm{CAX}$ & $y_{1}$ & 5 & & & $1\left(\mathrm{e}^{*}\right)$ & 1 \\
\hline P(IM) CRAS & 33 & 3 & 1 & & & \\
\hline$P(3,3) C A X$ & 27 & 8 & 1 & $1\left(e^{m}\right)$ & & 1 \\
\hline$P(3,3) C R A$ & 29 & 6 & 1 & & & 1 \\
\hline Suminary in $\%$ & \multicolumn{2}{|c|}{940} & \multicolumn{2}{|c|}{$3 \%$} & \multicolumn{2}{|c|}{$3 \%$} \\
\hline
\end{tabular}

ELECTRON FIELD TO THORACIC WALL

\begin{tabular}{|c|c|c|c|c|c|c|}
\hline Q0 Of prescribed dose; & $<25 \%$ & $25-49 \%$ & $50-74 \%$ & $75-84 \%$ & $85-94 \%$ & $\geq 95 \%$ \\
\hline$P(M M) C A X$ & & & 6 & & & 2 \\
\hline P(IM) CRAN & 2 & 2 & 1 & & 1 & \\
\hline$P(3,3) C A X$ & & 2 & 2 & 2 & 1 & 1 \\
\hline$P(3,3)$ CRAN & 2 & 2 & 1 & 1 & & \\
\hline Summary in $\%$ & & & & & & \\
\hline
\end{tabular}

See Matcrials and Methods section and figure 3 for definition of points $\mathrm{P}(\mathrm{IM})$ and $\mathrm{P}(3.3) ; \mathrm{CAX}=$ central slice; CRAN $=$ cranial slice $e^{-}=$electron field added to the medial part of the mastectomy scar.

Table 4. Number of treatment plans with the dose in $\%$ of the prescribed dose at the internal mammary lymph node chain for arm I (no IM-MS treatment).

\begin{tabular}{|c|c|c|c|c|c|c|}
\hline \multicolumn{7}{|c|}{ STANDARD TECHNIQUE } \\
\hline \% Of prescribed dose: & $<25 \%$ & $25-49 \%$ & $50-74 \%$ & $75-84 \%$ & $85-94 \%$ & $\geq 95 \%$ \\
\hline P(IM) CAX & 1 & & 1 & 1 & 3 & 33 \\
\hline P(IM) CRAN & & 1 & 1 & 1 & 4 & 35 \\
\hline $\mathrm{P}(3.3) \mathrm{CAX}$ & 1 & 3 & 6 & 4 & 6 & 19 \\
\hline$P(3,3)$ CRAN & 1 & 4 & 5 & 5 & 10 & 17 \\
\hline Sammary in $\%$ & \multicolumn{2}{|c|}{$7 \%$} & \multicolumn{2}{|c|}{$15 \%$} & \multicolumn{2}{|c|}{$78 \%$} \\
\hline \multicolumn{7}{|c|}{ ALTERNATIVE TECHNIQUES } \\
\hline \% Of prescribed dose: & $<25 \%$ & $25-49 \%$ & $50-74 \%$ & $75-84 \%$ & $85-94 \%$ & $\geq 95 \%$ \\
\hline$P(I M)$ CAX & & & & & 4 & 8 \\
\hline P(IM) CRAN & & & 1 & & 1 & 10 \\
\hline$P(3,3) C A X$ & & & 2 & 2 & 5 & 3 \\
\hline P(3.3) CRAN & & & 3 & 2 & 5 & 3 \\
\hline Sutnmary in \% & \multicolumn{2}{|c|}{$0 \%$} & \multicolumn{2}{|c|}{$20 \%$} & \multicolumn{2}{|c|}{$80 \%$} \\
\hline
\end{tabular}

Sec Materials and Methods secrion and Figure 3 for deffinition of poinss $\mathrm{P}(\mathrm{IM})$ and $\mathrm{P}(3,3) ; \mathrm{CAX}=$ central sliec; $\mathrm{CRAN}=$ cranial slice.

Table 5: Number of treatmen plans with the dose in of of the prescribed dose at the internal mammary lymph node chain for arm 2 (IM-MS treatment).

\subsection{Potential influence on trial outcome}

To calculate the possible impact of these potential dose variations on the final outcome of the trial, we estimated the potential dose variation in the trial based on the present accrual until now of $76 \%$ of the patients with breast conserving surgery and $24 \%$ with mastectomy. Projecting the above mentioned calculated dose variation to the IM-MS nodes over these figures, the potential dose variation to the IM-MS nodes may be 
estimated (Table 6). Combining the dummy run dose variation with the relative frequency of the use of the different treatment techniques, an estimation could be made of the potential dose variation to the IM-MS nodes in the total trial population for each randomization arm.

\begin{tabular}{lccc}
\hline \% Of prescribed dose: & $50 \%$ & $50-84 \%$ & $285 \%$ \\
\hline Arm 1 & 90 & 6 & 4 \\
Arm 2 & 5 & 16 & 79 \\
\hline
\end{tabular}

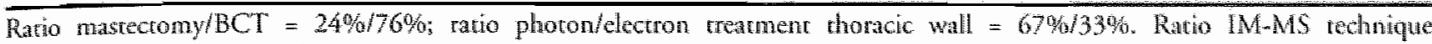
standard/alcernative $=78 \% / 22 \%$.

Table 6: Summary of the dose at the internal mammary lymph node chain for both treatment arms.

The principal endpoint of the trial will be overall survival. The expected survival rate in the control group, without IM-MS irradiation, is estimated to be $50 \%$ at 10 years. "The minimal potential benefit considered to be of clinical significance is a $5 \%$ gain in the 10 year survival to $55 \%$ in the arm with IM-MS irradiation (31). 'To illustrate the potential impact of the dose variation on survival, we assume, based on the typical sigmoidal cell survival curves, that a dose of $50 \%$ or less to the IM-MS region has no effect, a dose of $50-84 \%$ has a $10 \%$ effect, and a dose $85 \%$ or more has a full effect. Taking these assumptions into account, it can be calculated that the effect of the dose variation will be as follows: in case of a true 10-year survival benefit of 5\% with IM-MS irradiation, a benefit of only $3.8 \%$ would be measured due to a potential "overdose" in the control arm and a potential "underdose" in the treatment arm (Table 7).

\begin{tabular}{|c|c|c|c|c|}
\hline \multicolumn{5}{|c|}{ ARM I: BREAST/THORACIC WALL ONLY } \\
\hline$\%$ Of prescribed dose: & $0.50 \%$ & $50-84 \%$ & $85 \%$ & \\
\hline Dose to $1 \mathrm{M}-\mathrm{MS}$ region & 90 & 6 & 4 & \\
\hline 10-Year survival & 50 & 50.5 & 55 & \\
\hline Total & 45 & 3 & 2.2 & $=50.2 \%$ \\
\hline \multicolumn{5}{|c|}{ ARM II: BREAST/THORACIC WALL ONLY + IMC } \\
\hline \% Of prescribed dose: & $50 \%$ & $50-84 \%$ & $285 \%$ & \\
\hline Dose ro IM-MS region & 5 & 16 & 79 & \\
\hline 10-Year survival & 50 & 50.5 & 55 & \\
\hline Toral & 2.5 & 8.1 & 43.4 & $-54.0 \%$ \\
\hline
\end{tabular}

Table 7: Dose to internal mammary chain region

Notes: Theoretical influence of the actual dose at the IM-MS region as measured with the dummy run procedure on survivall, assuming that a dose $<50 \%$ to the IM-MS region has no effect, a dose of $50-84 \%$ has a $10 \%$ effect and a dose $\geq 85 \%$ has a full effect, based on a 10-year survival of $50 \%$ in the control group and a $5 \%$ absolute improvement from 50 to $55 \%$. The real difference in 10 -year survival of $5 \%$, at the level of significance, will be observed as a difference in 10-year survival of $3.8 \%$. 


\section{Discussion}

The EORTC 22922-10925 study investigates whether or not the elective irradiation of the internal mammary and medial supraclavicular lymph node chain has a positive impact on survival. To guarantee the accrual of a large number of patients, within an acceptable period of time, the participation of a large number of radiotherapy departments is necessary. Because of this, the treatment setup guidelines in the protocol were not very strictly defined, suggesting a simple treatment technique but allowing more complex treatment setup techniques as well. However, to limit the interinstitutional variability and to detect potential systematic protocol deviations due to ambiguities in the protocol prescriptions in an early phase of the trial, the Quality Assurance Committee initiated a dummy run procedure.

The dummy run procedure showed a significant number of, in most cases, small deviations from the protocol guidelines. Most of these deviations are due (1) to ambiguities in the protocol, (2) to disregarding the dose prescription guidelines of the ICRU $(37,38)$, and (3) for some institutions, to a lack of familiarity with irradiating the complex set of target volumes constituted by the IM-MS region and the breast/thoracic wall. A rather typical problem when using the standard technique for IM-MS irradiation together with breast/chest wall irradiation is the matching of the field junctions. Fourteen of the 25 institutions using the standard technique use a small overlap between the IMMS and the breast/chest wall fields, resulting in a superficial zone of overdose. Eleven institutions, in contrast, chose an exact match between the fields, resulting in a zone of underdose at depth. Most of the institutions claimed to individualize their choice of an exact match or an overlap based on patient- and tumor-related characteristics. Seven institutions used a modification of the standard technique to improve the dose distribution at the junction zone. The treatment plans provided by the 7 institutions using an alternative technique all showed an improved dose distribution at the field junctions.

As deviations in the irradiation of the IM-MS lymph node region itself will be of great importance for the final results of the trial, these were given more emphasis. Other deviations, which were judged to have only a limited impact on the endpoints of the trial, were reported as well, but were only considered as minor protocoll deviations.

The calculated doses at the points $\mathrm{P}$ (IM) and $\mathrm{P}(3,3)$ showed a significant variation from the ideal situation where a dose of $10 \%$ or less in Arm 1 and $95 \%$ or greater in Arm 2 should be expected. Because the sample size of the trial is based on significance at a $5 \%$ measured difference, this true $5 \%$ difference might, thus, lead to a (falsely) nonsignificant 
outcome of the trial. In other words, with the estimated dose variation, to measure a significant $5 \%$ difference in 10 -year survival, a true $6.6 \%$ benefit from (optimal) IM-MS irradiation would be needed. Similarly, the influence of the estimated interinstitutional dose variation on a hypothetical negative effect of IM-MS irradiation on long term survival due to side effects may be calculated. This will even aggravate the calculated differences. Of course, these hypotherical calculations, which can be done for every clinical trial, have to be considered with caution. The dummy run treatment plans only represent a theoretical patient and might give an erroneous impression of the actual treatment. We are well aware of the fact that we have to evaluate real patient data, which will lead to the final clinically relevant result of the trial.

As the dummy run was performed in the early phase of the trial, recommendations were given to the participants before a significant number of patients were accrued. These recommendations were given repetitively and on an individual basis after the review of the dummy run and during the plenary sessions of the meetings of the EORTC Radiotherapy Group. A letter with recommendations was sent around in November 1997 to all participants. Based primarily on the findings of the dummy run, a Revised Version of the trial protocol was prepared and distributed in May 1999. Further evaluation is ongoing in the third part of the QA program, which consists of an early eligibility and treatment compliance check in which adherence to the protocol can be checked in real (participating) patients. The potential dose variation at the IM-MS lymph node region will be calculated. This will allow us to check whether or not the participating institutions implement the recommendations based on the dummy run.

In the dummy run, a number of potential systematic protocol deviations that might lead to a false-negative result of the endpoint of the trial have been detected. It is expected that our suggestions and recommendations to improve the dose distribution will lead to a lower interinstitutional dose variation than encountered in the dummy run. This will improve the reliability of the final trial results.

\section{References}

1. Handley RS. Carcinoma of the breast. Ann R Coll Surg Engl 1975;57:59-66.

2. Handley RS. A surgeon's view of the spread of breast cancer. Cancer $1969 ; 24: 1232$.

3. Lacour J, Bucalossi $\mathrm{P}$. Caceres E, et al. Radical mastectomy versus radical mastectomy plus internal mammary dissection. Cancer 1976;37:206-214.

4. Levitr SH. Is there a role for post-operatiwe adjuvant radiation in breast cancery Beauriful hypothesis versus ugly facts: 1987 Gilbert H. Flexcher lecture. Int J Radiar Oncol Biol Phys 1988;14:787-796.

5. Veronesi $U$, Valagussa P. Inefficacy of internal mammary node dissecrion in breast cancer surgery. Cancer $1981: 47: 170-175$. 
6. Veronesi $U$, Cascinelli $N$, Greco $M$, et al. Proginosis of breast cancer patients afrer mastecromy and dissection of internall mannary wodes. Anin Surg 1985;202:702-707.

7. Veronesi U, Marubini $L$, Mariani $L_{\text {s }}$ et al. The dissection of internal mammary nodes does not improve the survival of breast cancer patients. 30-year results of a randomised trial. Eur I Cancer 1999;35:1320-1325.

8. Arkins $\mathrm{H}_{*}$ Hayward JL, Klugiman DJ, et al. Treatment for early breast cancer: A report after 10 years of a dinical trial. Br Med J $1972 ; 2: 423-429$.

9. Bruce J. Operable cancer of the breast: $A$ controlled clinical trial. Cancer $1957 ; 28: 1443-1452$.

10. Easson EC. Post-operative radiotherapy in breast cancer. In: Forrest APM, Kunkler PB, editors. Prognostic factors in breast caincer. New York: E \& S Livingstone, Lid; 1968. p. 93-102.

11. Fisher B, Slack $\mathrm{NH}_{0}$ Cawanaugh PJ, et al. Postoperarive radiotherapy in the rreatment of breast cancer: results of the NSABP clinical trial. Ant Surg 1970;172:711-730.

12. Wisher $B$, Wickerham DL, Deursch $M$, et al. Breast rumour recurrence following lumpectomy with and without breast irradiation: An overview of recent NSABP findings. Sem Surg Oncol 1992;8:153-160.

13. Hamilon T, Langlands AO, Prescorr RJ. The treatmenr of operable cancer of the breast: A clinical trial in the Southeast tegion of Scotland. Br I Surg 1974;61:758-761.

14. Kata $S$, Johansen $H$. Breast cancer-A comparison of the results of simple mastectomy with postoperative roentgen irradiation by the MeWhirter method with those of extended radical mastectomy. Acta Radiol 1959;188(Suppl.): 155-161.

15. Patterson R, Rusgell M. Clinical trials in malignant disease: Part IIll. Breast Cancer. Evaluation of postoperative radiotherapy. J Fac Radiol 1959:10:175-180.

16. Fowble $B$, Hanlon $A$, Fredman $G$, et al. Internal mammary node irradiation neither decreases distant metastases nor improves surviwal in stage I and II breast cancer. Int J Radiat Oncol Biol Phys 2000;47:883-894.

17. Cuzick I" Stewart $H$, Peto $R$, et al. Overview of randomized trials of postoperarive adjuvant radiotherapy in breast cancer. Cancer Treat Rep 1987;71(1):15-25.

18. Cuzick J. Stewart $H$, Rutqwist $\mathbb{L E}$, er al. Cause-specific mortality in long-term survivors of breast cancer who participated in trials of radiotherapy. I Clin Oncol 1994;12:447-453.

19. Stjernsward ]. Decreased survival. related to irradiation postoperatively in early operable breast cancer. Lancet 1974:2:1285-1286.

20. Harris JR, Hellman S. Put the "Fockey stick" on ice. Int J Radiar Oncol Biol Phys 1988;15:497-499.

21. Overgaard M, Hansen PS, Overgaard J, et al. Postoperative radiotherapy in high-risk premenopausal women with breast cancer who receive adjurvant chemotherapy. N Engl J Med 1997;337:949-955.

22. Overganded M, Jensen M-J, Overgaard J, er al. Posroperative radiotherapy in high-risk postmenopausal breastancer patients given adjuvant Tamoxifen: Denish Breast Cancer Cooperative Group DBCG $82 \mathrm{crandomised} \mathrm{trial.}$ Lancet 1999; 353:1641-1648.

23. Ragaz l. Jakkson $S M, L e N$, er al. Adjuvant radiotherapy and chemotherapy in node-positive premenopausal woirict with breast cancer. N Jing J Med 1997;337:956-962.

24. Højris 1. Owergaard M, Christensen JJ, et al. Morbidity and mortality of ischemic heart disease in high-risk breastcancer patients after adjuvant post-mastectomy systemic treatment with or without radiotherapy: Analysis of DBCG $82 \mathrm{~B}$ and $82 \mathrm{C}$ randomised trials. Lancet 1999;354:1425-1430.

25. Kaja H, Mauna P. Tangential breast irradiation with or without internal mammary chain irradianion: Results of a randomized rrial. Radiother Oncol 1995;36:172-176.

26. Edland RB. Presidential address: Does adjuvant radiotherapy have a role in the postmastectomy management of parients with operabte breast cancer-Revisited. Int I Radiat Oncol Biol Phys 1988;15:519-535.

27. Fetelier $\mathrm{GH}$, Montigue ED. Does adequate irradiation of the internal mammary chain and supraclavicular nodes improve survival rates? Int J Radiat Oncol Biol Phys 1978:4:481-492.

28. wan "Tienhoven G, van Bree NAM, Mijnheer BJ, et al. Quality assurance of the EORTC trial 22881/10882: "Assessment of the role of the booster dose in breast conserving therapy:" The dummy run. Radiother Oncol $1991,22: 290-298$.

29. Hurkmans $\mathrm{CW}$, Borger $] \mathrm{H}$, Bos LJ, et all. Cardiac and lung complication probabilities after breast cancer irradianion. Radiother Oncol 2000;55:145-151.

30. Rutquist LE, Lax I, Fornander T, et al. Cardiovascular mortalicy in a randomized trial of adjuvant radiation therapyversus surgery alone in primary breast cancer. Int J Radiat Oncol Biol Phys 1992;22:887-896. 
31. Van den Bogaere W, Struikmans $H_{3}$ Fourquet $A$, al. Internal mammary and medial supridavicular (IM-MS) lymph node chain irradiation in stage I-1II breast cancer. Aphase III randomised trial of the EORIC, protocol 22922/10925, May 1996, revision May 1999. Brussels EORTC 1999.

32. Dusserre A, Garavalia G, Giraud J-Y. er a. Quality assurance of the EORTC radiotherapy riat 22863 for prostaric cancer: The dummy run. Radiother Oncol 1995;36229 234.

33. Thwaites D. Quality assurance into the next century. Editorial. Radionter Oncol 2000;54:vii-ix.

34. Valley J-F, Bernier ], Tercier P-A, er al. Quality assurance of the EORTC radionthempy rrial 22931 for hewd and neck carcinomas: The dummy run. Radiother Oncol 1998:47:37-44.

35. Bolla $\mathrm{M}$, Bartelink $\mathrm{H}$, Garavaglia $\mathrm{G}$, et al. EORTC guidelines for writing protocols for dinical trials of radiotherapy. RadiorherOncol 1995;36:1-8.

36. wan Tienhoven G, Mijnheer B], Bartelink H, al. Quality assurance of the EORTC rial 22881/10882; "Boost versus no boost in breast conserving therapy: An overview." Strahlenther Onkol 1997;173:201-207.

37. International Commission on Radiation Units and Measurements (ICRU). ICRU report 50. Preseribing, recording, and reporting photon beam therapy. Bethesda, MD: International Commission on Ractiation Units and Measurements; 1993.

38. International Commission on Radiation Unirs and Measurements (ICRU). ICRU report 62. Prescribing, recording, and reporting photon beam therapy (Supplement to ICRU report 50). Bethesda, MD: International Commission on Radiation Units and Measurenents.

39. Pierce LJ, Lichter AS. Postmastectomy radiotherapy: More than locoregional control. I Clin. Oncol $1994 ; 12: 444-446$.

40. Struikmans H, van Rijk P. Optimizing radiotherapy of the internal mammary chain in breast carcinoma by scintigraphy. Radiother Oncol 1996;41:15-20. 



\section{CHAPTER 7}

A glance on quality assurance in EORTC study 22922 evaluating techniques for internal mammary and medial supraclavicular lymph node chain irradiation in breast cancer.

Yolande Lievens, Philip Poortmans, Walter Van den Bogaert.

Radiother, Oncol. 60: 257-265, 2001. 


\section{Abstract}

Purpose: To evaluate the irradiation techniques used for the irradiation of the internal marnmary and medial supraclavicular lymph node chain (IM-MS) in the EORTC 22922 study, which evaluates its impact on survival in stage I-III breast cancer patients with axillary node invasion and/or central or medial location of the primary tumour.

Materials and Methods: The analysis was performed based on the dummy run data of the Quality Assurance Programme of the study. A standard irradiation technique was proposed within the study protocol, and the use of other treatment set-up techniques was allowed.

Results: By March 2000, 39 centres from 12 different countries had participated in the study; 32 of these had already fulfilled the dummy run procedure. No centres had to be excluded from the study. Seventy-eight percent of the centres are using the standard irradiation technique, $64 \%$ of these with minor variations. Twenty-two percent of the centres developed an alternative irradiation technique. The remarks to the centres using the standard set-up were most often related to the junction problem and the possible under- or overdosage in the target volumes. The remarks to the centres with alternative techniques most often concerned the possible enhanced dose to the lungs and the heart.

Conclusions: In a multi-centre trial an easy irradiation technique applicable in a large number of centres should be provided. A quality assurance programme allows early detection of possible problems with treatment planning and delivery. The analysis of the dummy run data showed that if the recommendations of the Quality Assurance Committee are applied, both standard and alternative IM-MS irradiation techniques produce acceptable dose distributions.

Keywords: Irradiation technique; Internal mammary and medial supraclavicular lymph node chain; Quality assurance 


\section{Introduction}

The irradiation of the internal mammary lymph node chain in post-operative breast cancer patients remains an object of controversy and debate.

Adjuvant loco-regional radiotherapy in breast cancer has sufficiently been proved to decrease local recurrence $[1,3,7,8,10,15,23]$. Still, in older radiotherapy studies this advantage did not translate into an improved survival $[4,12]$ because the reduced odds of dying from breast cancer were counterbalanced by an increased risk of death from other causes, mostly related to an increased cardiac mortality [5]. It is onlly very recently that three randomized trials could demonstrate the positive impact of loco-regional irradiation on survival $[21,22,26]$, without compromising survival due to cardiac side effects [14]. Although it has been suggested that the effect on survival in these studies may have been mainly related to the irradiation of the internal mammary lymph node chain, it is difficult to prove the advantages of the elective irradiation of these lymph nodes.

In early surgical series metastases in the internal mammary lymph nodes have been described to range between $4 \%$ in axillary node-negative patients with upper lateral tumour location and $72 \%$ in tumours of the lower medial quadrant of the breast with histologically proven positive axillary lymph nodes $[11,17,20,33]$. In a recent literature overview based on surgical series as well as on data from sentinel lymph node mapping [9], the involvement of the internal mammary chain was estimated to be much lower in today's breast cancer patients, diagnosed by screening mammography. According to this overview, internal mammary lymph node involvement over $25-30 \%$ is associated with large primary tumours in the breast or high numbers of involved axillary nodes.

Surgical series investigating the impact of the dissection of this lymph node region were not able to demonstrate any survival advantage $[17,33]$. To date only two randomized radiotherapy trials have focused on the possible benefit of adjuvant internal mammary and medial supraclavicular irradiation alone $[16,28]$. No differences in outcome have as yet been shown, but in both studies follow-up is still too short. In the Finnish study, moreover, the number of patients is too limited to demonstrate any effect [16]. The Lyon study was designed to demonstrate a $10 \%$ survival difference, which could be hard to obtain [28].

In May 1996 a large multi-centre trial was initiated by the EORTC to further elucidate the problem of improved outcome - loco-regional control and survival - after the irradiation of the internal mammary and medial supraclavicular lymph node chain (further referred to as IM-MS) in patients with localized breast cancer. The principal goal 
of this EORTC 22922/10925 trial [31], randomizing between the irradiation of the IMMS or not, is to solve the controversy concerning its role in terms of survival: are the benefits from possible improved tumour control jeopardized or not by the negative influence from possible side effects?

To prove or rulle out an existing difference in survival from this adjuvant treatment, large numbers of patients are needed. In order to accrue a large and representative sample of the average European patient through such a multi-centre trial, a simple standard treatment set-up was chosen for the IM-MS irradiation in patients after mastectomy or after lumpectomy. More complex treatment set-ups were also accepted, provided that these irradiation techniques met the basic trial recommendations.

In multi-centre trials it is necessary to perform quality assurance procedures in order to obtain reliable information about a centre's ability to cope with the protocol prescriptions and its compliance to the protocol. Moreover, it allows ambiguities in the protocol prescriptions and possible systematic protocol deviations to be readily detected. In this article the IM-MS irradiation techniques used by the participating centres and the possible problems related to the use of these techniques are presented. The presented data are derived from the dummy run data of the EORTC 22922/10925 trial Quality Assurance Project, focusing on the internal mammary part of the IM-MS irradiation.

\section{Materials and methods}

\subsection{The Quality Assurance Project}

As it is customary in studies performed in the EORTC radiotherapy group, the Quality Assurance Project of trial 22922/10925 has been divided into two consecutive parts: the dummy run (Part $\mathrm{I}$ ) is followed by an individual case review early in the accrual phase of the trial (Part II).

In the dummy run procedure the contributing centres are asked to perform treatment planning according to the EORTC 22922 protocol under two different circumstances: the treatment volume either limited to the thoracic wall/ breast or including the internal mammary and medial supraclavicular lymph node regions. A set of three transverse slices of a patient after a mastectomy on the left side and after a lumpectomy on the right side is provided to each centre. The dummy run procedure is to be performed for each of the different techniques the centre intends to use for the treatment of patients within the triall.

The replies of the participating institutions are analyzed, qualitatively as well as 
quantitatively, in order to quickly detect ambiguities in the protocol prescriptions as well as technical impossibilities that may hamper protocol compliance. After the evaluation of the received data by the quality assurance team, a letter with comments on the acceptability of the plans is sent to the participating centre.

In the second part of the Quality Assurance Project, actual treatment plans will be evaluated. This work, which is still ongoing, will enable us to evaluate the effect of the dummy run procedure on the dose variation between the participating centres and between different patients.

\subsection{The standard irradiation technique}

The patient is treated in supine position, which is reproducible during simulation and treatment. The lateral border of the field is defined at the middle of the clavicle, and the upper border is $3 \mathrm{~cm}$ above the head of the clavicle. The medial field border lies $1 \mathrm{~cm}$ across the midline, and the lateral field border of the internal mammary part of the field is $5 \mathrm{~cm}$ lateral from the midline. The lower border of the fourth rib defines the lower field border; in medial inferior quadrant lesions the inferior border may have to be lowered in order to include the fifth intercostal space. The prescribed dose is $50 \mathrm{~Gy}$ in 25 fractions of $2 \mathrm{~Gy} ; 26 \mathrm{~Gy}$ is delivered with photons (minimum energy of Co-60 and maximum energy of $10 \mathrm{MV}$ ), and $24 \mathrm{~Gy}$ is delivered with electrons. To cover the clinical target volume (CTV) with at least the $95 \%$ isodose line for photons and $85 \%$ for electrons, the prescription point for photons is defined at $3 \mathrm{~cm}$ depth and the electron energy is chosen between 12 and $14 \mathrm{MeV}$. With the standard technique as described here, the planning target volume covers the internal mammary nodes, which in more than $85 \%$ of the patients lie within $4 \mathrm{~cm}$ lateral to the midline and extend up to $4 \mathrm{~cm}$ depth $[24,27]$. Fig. 1 shows the dimensions and position of the standard IM-MS field, with a block at the upper medial border to protect the laryngeal structures. When individual localization of the IM-MS lymph node region is performed, participating centres are encouraged to individually adapt the beam energy and prescription depth.

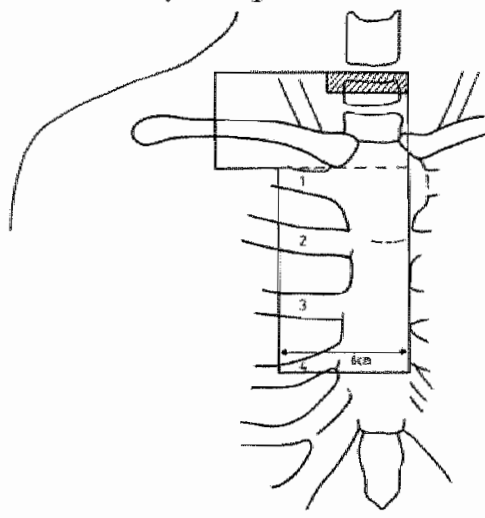

Figure 1: Standard irradiation set-up of the EORTC 22922 study. 


\subsection{Data analysis}

The following data were analyzed: the inclusion rate and the country and ciry of the centre, the completion of the Quality Assurance Programme (first and/or second part) by the centre and the remarks of the Quality Assurance Committee following the dummy run procedure per centre. Furthermore, the techniques in use were described, including deviations from the proposed standard technique and centre-specific techniques as well as the hereby potentially induced problems.

The data were organized with a File Maker Pro programme. Descriptive analysis was performed to summarize the data.

\section{Results}

\subsection{Participating centres}

By March 2000, 39 centres from 12 different countries had participated in the study; 35 of these centres are active, i.e. have already accrued patients in the study. Thirty-two centres completed the dummy run, of which ten also completed the second part of the Quality Assurance Program with individual case reviews of the first six patients (three in each treatment arm) enrolled in the study. Six centres are active without having fulfilled the dummy run procedure. Further results will only include centres that have completed the dummy run procedure (Appendix A).

In two centres the dummy run showed such extensive irradiation of the normal tissues (especially lung) for one proposed technique that this was not accepted for treatment of patients within the treatment protocol. In one centre other techniques were proposed within the same dummy run and accepted, and in the other centre a new dummy run was performed and accepted. No centres have been excluded from the trial due to unacceptable treatment techniques or protocol deviations.

\subsection{Irradiation techniques used}

\subsubsection{Thonacic wall irradiation}

The majority of the centres use tangential photon fields for the irradiation of the thoracic wall ( 18 out of $32 ; 56 \%$ ), and nine centres ( $28 \%$ ) use electrons in a direct field set-up. No data are known for three centres and the remaining two centres restrict the inclusion of patients to breast-conserving situations or to the irradiation of the IM-MS only. Amongst the nine largest centres (>50 patients included), eight use tangents, and only one centre uses electrons. 


\subsubsection{M-MS irradiation}

Table 1 gives an overview of the IM-MS irradiation techniques used. Twenty-five centres $(78 \%)$ use the standard technique as described in the trial protocol and in the materials and methods section. Sixteen of these slightly adapt this standard set-up (Table 2). The most frequently used adaptation - in nine centres - is a limited inclination of $10-15^{\circ}$ of the IM-MS field towards the tangential fields, in order to overcome an underdosage at the junction of the fields. Of these, one centre only inclines the electron IM-MS irradiation, and one only the photon IM-MS irradiation. In two centres a special junction technique is used: the technique of Lagendijk and Hofman [18] and that of Lebesque [19]. Three centres use a shifting junction between the IM-MS field and the medial edge of the breast/thoracic wall field. The Quality Assurance Committee accepted all of these centre-specific adaptations.

\begin{tabular}{lcc}
\hline M-MS technique & Number of centers & Percentage of centers \\
\hline Standard (unmodified) & 9 & 28 \\
Standard (nodified) & 16 & 50 \\
Alsernative & 7 & 22 \\
\hline
\end{tabular}

Table 1: Distribution of the IM-MS techniques used.

An adaptation of the width of the IM-MS field (either wider or narrower, each seen in one centre) was only judged to be acceptable if specific localization techniques are used. The remaining adaptations were not accepted and were corrected before inclusion of patients in the trial. Four centres positioned the IM-MS field at midline, i.e. too far to the contralateral side, thus compromising the dose to the parasternal lymph nodes. In six centres the energy used in the IM-MS field did not conform to the protocol: in three cases the proportion between electrons and photons was changed, two centres proposed only photon fields, and one centre proposed only electron irradiation.

\begin{tabular}{lc}
\hline Way of adaptation & Number of centres \\
\hline Inclination M-MS & 9 \\
Adapted electrons or phorons & 6 \\
Shifr central planc & 4 \\
Shifting junction & 3 \\
Width IM-MS & 2 \\
Junction teclanique & 2 \\
\hline
\end{tabular}

Table 2: Different ways of adapting the standard technique. 
Seven centres use an alternative technique individualized to the specific patient based on the treatment planning $\mathrm{CI}$ scan. Two of these centres use both the standard and an alternative technique, the choice in a specific patient being motivated by the localization of the tumour and the anatomy of the patient. In the five other centres only the centrespecific technique is used.

All the alternative techniques have the following set-up in common: the mediolateral tangential field is enlarged in order to encompass the breast/thoracic wall as well as the parasternal lymph node chain, whereas the lateromedial tangential field is delimited by the breast/thoracic wall target volume. Since this enlarged medial field is insufficient to ensure a correct dose to the parasternal region, an additional field is delivered. Its energy (electrons, photons or a mixture of both), inclination, localization and specification are defined on the basis of the three-dimensional dose calculation and vary amongst the centres. Fig. 2 demonstrates the set-up of a representative technique.

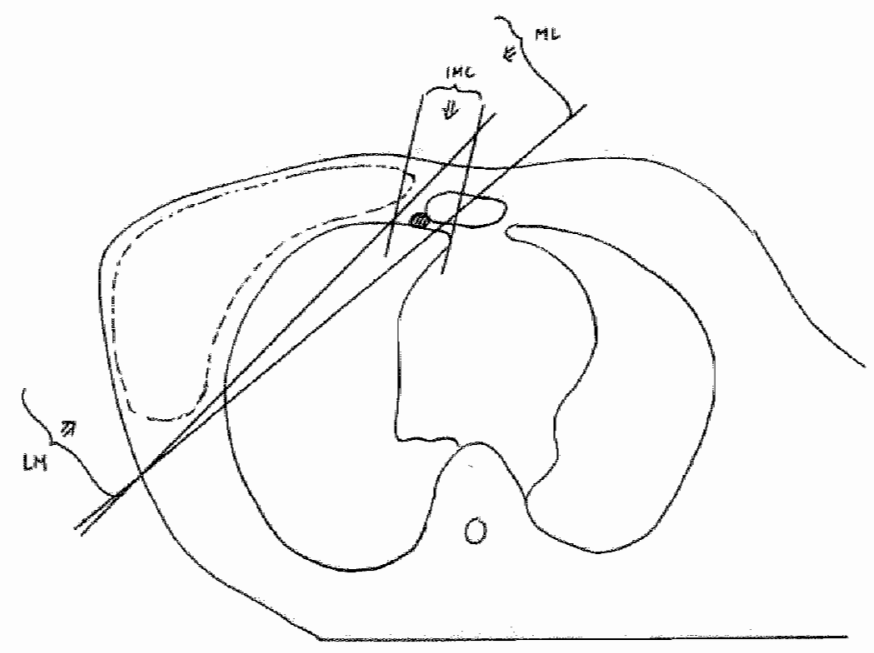

Figure 2: Example of an alternative irradiation technique

Fig. 3 shows the frequency of use of the standard technique versus the alternative techniques. If all 32 active centres are considered, $78 \%$ use the standard technique, of which $44 \%$ accept an overlap at the junction zone, against 34\% not accepting an overlap. Only seven centres use the alternative technique (22\%), of which two sometimes still use the standard technique. When taking only the largest centres into consideration, i.e. the centres that included more than 50 patients, the proportion of centres using the alternative technique increases (three out of nine; $33 \%$ ). 


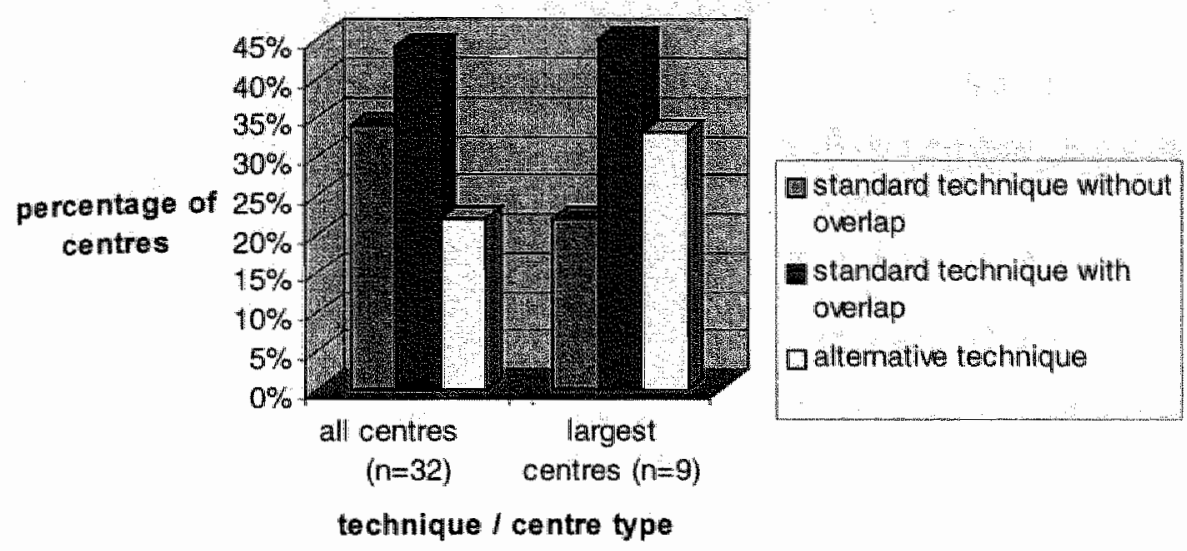

Figure 3: Distribution of the wadiation techniques of the IM-MS.

\subsection{Remarks of the Quality Assurance Committee}

Table 3 shows the remarks of the Quality Assurance Committee concerning potential systematic protocol deviations. They have been divided into general remarks, remarks concerning the irradiation of the breast/thoracic wall and problems specifically related to the irradiation of the IM-MS. Distinction has further been made according to the IMMS irradiation technique used by the radiotherapy centre. Centres using the standard technique have been compared to centres using the alternative technique or both alternative and standard techniques.

\subsubsection{General remarks}

The general remarks were overall well balanced between the two groups. Still, some problems were predominantly encountered in centres using the standard technique: dose prescriptions not conforming to the ICRU guidelines (32 vs. $1.4 \%$ ), normalizations not performed on the whole plan (28 vs. 14\%), plans without lung density correction (16 vs. $0 \%)$ and no monitor unit calculations provided (24 vs. $14 \%)$.

\subsubsection{Remarks on the irradiation of the breastithoracic wall}

In 14 centres the minimal dose to (part of) the target volume turned out to be less than 95\% of the prescribed dose; this problem was more frequent with the alternative technique $(71 \%)$ than with the standard technique $(36 \%)$. In 13 centres this too low minimal dose was at least partly due to narrow margins around the CTV.

An unintended high dose ( $>25 \%$ ) to the IM-MS region in case of isolated irradiation of the breast or the thoracic wall was more frequently seen in centres using the standard technique (40\%) than in those using allernative approaches $(29 \%)$. 


\subsubsection{Remarks on the irradiation of the IM-MS: IM-MS technique and dose bomogeneity}

The use of an incorrect length or width of the IM-MS field was as frequently seen in both groups, as well as the erroneous extension of the bolus of the scar into the IM-MS field, resulting in an insufficient depth dose.

Problems with the junction of the IM-MS and the breast/thoracic wall field were predominant in the standard technique. An overlap resulting in a hot-spot (a dose of $>110 \%$ ) was seen in $48 \%$ of these centres using the standard technique, an exact match or an incorrectly positioned IM-MS field (in 40 and 24\%, respectively), leading to an underdosage (a dose of $<95 \%$ ) in the target volume.

The unacceptable selective use of electrons or photons for IM-MS irradiation was only encountered in the standard technique.

\begin{tabular}{|c|c|c|}
\hline Type of arradiation tised (w) & $\begin{array}{l}\text { Standard } \\
(n=25)(\%)\end{array}$ & $\begin{array}{l}\text { Altern } \\
n=7\end{array}$ \\
\hline \multicolumn{3}{|l|}{ Genluryititimarks } \\
\hline Missing asadose lines (20) & $15(60)$ & $5(71)$ \\
\hline Dose descripton not ICRU' $(9)$ & $8(32)$ & $1(14)$ \\
\hline Normalization not or whole piltan (8) & $7(28)$ & $1(14)$ \\
\hline No monitor units catcullations (7) & $6(24)$ & $(14)$ \\
\hline No plans withoul H-MS (7) & $500)$ & $2(29)$ \\
\hline Dose prescription nol $25 \times 2 \mathrm{~Gy}(\mathrm{~s})$ & $4(16)$ & I(1) \\
\hline Platning wuthout clectrons ( 4 ) & $2(8)$ & $2(29)$ \\
\hline No lung denrsity correction $(4)$ & $4(16)$ & $0(0)$ \\
\hline No drawing of internal sinsethres (2) & I (4) & $1(14)$ \\
\hline Magniffention filctor platin (2) & $2(B)$ & $0,(0)$ \\
\hline Only inclusion of spacitic subgroups (2) & $2(8)$ & $0(0)$ \\
\hline Missing beam data (2) & (4) & $1(14)$ \\
\hline \multirow{2}{*}{ Scpantite edectron and photom plates (1) } & $(4)$ & $0(0)$ \\
\hline \multirow{2}{*}{\multicolumn{3}{|c|}{ 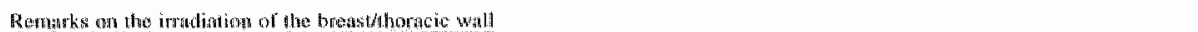 }} \\
\hline & & \\
\hline 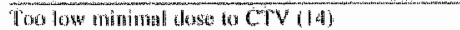 & $9(36)$ & $5(7)$ \\
\hline 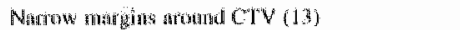 & $10(40 \mathrm{~g}$ & $3(43)$ \\
\hline 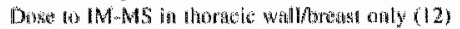 & $10(40)$ & $2(29)$ \\
\hline Tangential listles in $180^{\circ}(3)$ & $3(12)$ & $0(0)$ \\
\hline 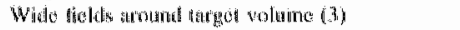 & $3(12)$ & $0(0)$ \\
\hline Whong wed dae factor (2) & $2(8)$ & $0(0)$ \\
\hline
\end{tabular}

Rernarks on thw Jualiation or the HM MS

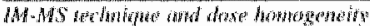

Overtap: mind hur

$12(48)$

$\left.2(2)_{1}\right)$

Finct wateh; mind tiadkrelossuge (1.1)

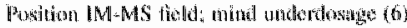

Widtu M-MS (4) 


\subsubsection{Remarks on the irradiation of the IM-MS: dose prescription of the IM-MS}

Problems related to the dose prescription were evenly distributed between both groups.

\subsubsection{Remarks on the imadiation of the $I M-M S$ : dose to nomal tisnes}

All seven centres using complex centre-specific IM-MS irradiation techniques were identified as being at risk of irradiating a substantial volume of lung and heart up to a significant dose (50-70\% of the prescribed dose). Three of these centres produced dose volume histograms of their techniques, enabling quantification of the volume of lung irradiated. In the different plans provided by these centres, respectively 18-33, 38 and 20$28 \%$ of the homolateral lung was irradiated up to a dose of 20 Gy. One centre also provided data on the volume of heart irradiated. The maximal dose to the heart in combined left-sided breast and IM-MS irradiation was 25 Gy. The standard technique with an inclined IM-MS field, used in nine centres, could potentially result in a slightly higher lung dose as well. Following the dummy run data, this was judged to be the case only in one centre.

Although 14 standard technique centres were found to allow some overlap between the internal mammary field and the breast or thoracic wall fields, a high skin dose (up to $150 \%$ ) was proportionally not more frequent in these centres (44\%) than in the centres using an alternative technique ( $43 \%)$.

\section{Discussion}

The EORTC 22922/10925 study [31] investigates whether or not the elective irradiation of the internal mammary and medial supraclavicular lymph node chain has a positive impact on survival. In order to recruit the large number of patients necessary to solve this question, an easy irradiation technique, applicable in a large number of centres, is necessary. The standard technique as proposed in the study fulfils these requirements: this simple and straightforward technique produces an adequate dose distribution to the target volume in the majority of the patients, while limiting the dose delivered to heart and lung. This is accomplished by the use of the mixed beam IM-MS technique and by defining the lower limit of the IM-MS field at the lower border of the fourth rib (except for tumours of the lower quadrant), thus sparing the largest part of the heart. The majority of the centres participating in the study $(78 \%$ ) are using this standard technique, be it in some cases with minor variations.

Alternative techniques were allowed, provided that these fulfil the basic trial recommendations. Twenty-two percent of the centres adopted an alternatiwe irradiation 
technique, representing one-third of the large centres participating in the trial.

The Quality Assurance Committee initiated a dummy run procedure to rule out potential systematic protocol deviations and to detect mistakes of particular institutions. A detailed qualitative and quantitative overview of all the possible deviations, as well as their potential impact on the final outcome of the trial, are discussed elsewhere [25]. In Table 3 these deviations are analyzed according to the IM-MS irradiation technique used.

General problems and problems related to the irradiation of the breast or thoracic wall were easily solved by distributing recommendations or clarifications of parts of the protocol to the participating centres. Some deviations were accepted as minor protocol deviations. Of interest is that even if not all of these problems are technique-specific, more corrections had to be made in the centres using the standard technique. This may be related to the fact that centres newly implementing the standard technique in order to participate in the study were less familiar with the technical difficulties than centres who developed their own irradiation technique. Moreover, the additional effort associated with the individual treatment planning of the alternative techniques may limit these minor deviations.

The remaining problems concerned alterations of the IM-MS irradiation technique, possibly resulting in dose inhomogeneity or in enhanced dose to the critical organs. Most of these were also easily corrected or judged to have no major impact on the endpoints of the trial. In two cases the particular deviations showed a too high dose to critical organs and were therefore unacceptable in the scope of the endpoints of the trial. Because in these particular institutes another suitable technique was present, they were not excluded from the study.

Deviations of the protocol related to the junction of the breast or thoracic wall field and the IM-MS field were predominantly seen in centres using the standard technique. Sixteen centres (Table 2) proposed different solutions.

An inclination of the IM-MS field towards the tangential fields aims at minimizing the 'cold triangle' that is inevitably seen if a sharp junction of the IM-MS with the tangential fields is chosen. This 'cold triangle' increases in volume with increasing breast volume and with decreasing distance between the midline of the patient and the medial border of the breast. In laterally implanted breasts of small to median size, this zone of underdosage remains negligible provided that the tumour bed is not localized in this region. Although a potential drawback of the inclination is a higher dose to the lungs and possibly to the heart, this risk was estimated to be limited after analysis of the dummy run data and the inclination was thus accepted as a minor protocol deviation. In three centres a shifting 
junction technique is used. In this approach the junction between the IM-MS and the breast or thoracic wall field is shifted from day to day, thus avoiding a high dosage to a small area. Other special ways of junctioning the IM-MS with the tangential field (used in two centres) have been described in the literature as the junction techniques of Lagendijk and Hofman [18] and Lebesque [19]. Fourteen centres allow an overlap between the lateral border of the internal mammary field and the tangential field. This practice increases the risk of late skin reactions - match line fibrosis and telangiectasia as a consequence of an overdosage to the skin [32]. Since it does not impact on survival it was accepted by the Quality Assurance Committee.

The IM-MS field was shifted towards the contralateral side in four centres. Since this may result in insufficient coverage of the IM-MS region, these centres were asked to abandon it. If in the concomitant breast/thoracic wall and IM-MS irradiation the simulation procedure is started by delineating the breast or thoracic wall field, the risk exists that the IM-MS field needs to be shifted contralaterally in order to avoid overlap between the fields. It is therefore strongly recommended to start the concomitant simulation with the delineation of the IM-MS field.

Seven participating centres use an alternative field set-up, which is individualized to the specific patient based on the treatment planning CT scan. These techniques optimize the dose distribution in the junction zone. This is especially advantageous in patients where the breast tissue extends far medially or when the tumour is located in the superior internal quadrant of the breast. Another advantage of the use of individual anatomical information is that the treatment field dimensions, energies and beam weights can be individually adjusted in order to encompass the CTV with sufficient margins. Common to these techniques is the use of a larger mediolateral field and an inclined parasternal field. This explains the possible disadvantage of a higher dose - and thus side effects - to the lungs and possibly to the heart and the skin related to these techniques. In the dummy run procedure one centre proposing a more complex IM-MS irradiation technique was indeed identified as irradiating a too large volume of lung and heart up to a significant dose $(50-70 \%$ of the prescribed dose). This centre was discouraged from using this set-up for the study. In three other centres dose volume histograms of the proposed techniques were produced, quantifying the volume of homolateral lung irradiated. These data are based on only three CT slices of the homolateral lung provided by the dummy run procedure. This makes these dose volume histograms difficult to interpret in comparison to the literature, where it is standard to evaluate the side effects after pulmonary irradiation, based on the total lung volume. One centre also produced a dose volume histogram of the heart for the combined irradiation of the breast and the 
IM-MS at the left side. The study of Hurkmans et al. [13] suggested that one should not have too many concerns about a higher dose to the heart and the lung in alternative techniques. In their dosimetric study they compared the standard technique, allowing 5 mm overlap between the internal mammary nodes and the tangential fields, to an alternative technique, aligning the internal mammary field to the tangential fields. In both techniques $70 \%$ of the dose was delivered by electrons, and $30 \%$ was delivered by 6-8 MV photons. Doses to the heart tended to be lower with the new technique, be it at the expense of small increases in lung dose [13].

Six centres changed the proportion of electrons and photons within the IM-MS field, without individual anatomical information. One centre even planned its IM-MS field only with electrons. Although this technique will of course diminish the side effects on the deeply localized organs (oesophagus, heart and lungs), it will on the contrary enhance the side effects to the skin. The inverse is true when only photons are used for the IMMS field irradiation, as proposed by two centres. The resulting dose to the underlying deeply localized organs (heart and lung, as well as oesophagus) will be too important and enhance the risk of important late side effects to these organs. Because of the possible impact on the major endpoint of the study (survival), this practice was discouraged.

Four centres adapted the width of the IM-MS field. It was specified that this was only acceptable if an individualized localization of the internal mammary lymph node chain (e.g. by CT scan or ultrasonography) is performed. Bentel et al. [2] showed that the CT scan is an accurate localization method. They observed a median depth of the internal mammary vessels of $2.4 \mathrm{~cm}$ (range $1-6 \mathrm{~cm}$ ) and a variation in lateral distance berween 1.7 and $3.7 \mathrm{~cm}$ (median $2.5 \mathrm{~cm}$ ). Scintigraphy may be an even more specific method for localizing the parasternal nodes [6]. Struikmans and van Rijk [30] observed a wide range in the localization of this lymph node chain in their scintigraphic study in 320 parients. They conclude that the important anatomical variations may render the use of fixed target volumes in the IM-MS radiotherapy inappropriate. Patient-specific localization allowing individualized treatment planning, leading to a better coverage of the target volume while optimally sparing the critical organs, should therefore be encouraged $[2,29]$, not only in the alternative technique but also when the standard technique is used.

The alternative techniques are more complex and time-consuming - and therefore maybe also more cost-consuming - than the standard set-up, rendering them nowadays unsuitable for standard use in all participating centres. However, when analyzing the centres with the highest inclusion rate, it turns out that they more frequently use these alternative techniques as compared to the totality of the centres. 
It has sufficiently been shown that treatment techniques and delivery play an important role when it comes to avoiding side effects that could jeopardize survival advantages $[4,5]$. That an improvement of survival is possible with post-operative radiotherapy in breast cancer patients has been shown in recent randomized studies $[21,22,26]$. If this is also true for the elective irradiation of the internal mammary and medial supraclavicular lymph node chain remains to be tested. The only randomized trials investigating this topic $[16,28]$ until now did not show any difference but lack the statistical power to do so or aim for unrealistic high survival advantages. Hopefully the results of this large randomized EORTC study will be able to solve the controversy.

\section{Acknowledgements}

The authors thank the following members of the Quality Assurance Programme of the study: Philip M.P. Poortmans, Jack L.M. Venselaar, Vernon Vlaun, Karin A. De Winter, Marleen H. Van der Hulst (Dr. Bernard Verbeeten Instituut, Department of Radiotherapy, Tilburg, The Netherlands); Henk Struikmans, Jan J.W. Lagendijk (University Medical Centre, Department of Radiotherapy, Utrecht, The Netherlands); Coen W. Hurkmans, Ben J. Mijnheer (Antoni van Leeuwenhoek Huis, Department of Radiotherapy, Amsterdam, The Netherlands); J. Bernard Davis (University Hospital, Department of Radiotherapy, Zurich, Switzerland); Dominique Huyskens, Walter F.C. Van den Bogaert (University Hospital, Department of Radiotherapy, Leuven, Belgium); Geerrjan van Tienhoven (Academic Medical Centre, Department of Radiotherapy, Amsterdam, The Netherlands).

\begin{tabular}{|c|c|c|c|}
\hline City. & Country & Institution & Local contact persons \\
\hline Brukelles & Belginum & Universicy Hospial Sann-Luc & C.Kirkhove \\
\hline Leaven & Belgium & Uniwersiry Hospital & W. wan den Bogater, D.Huysikens \\
\hline Whilrijk & Belgium & A2 Sine $A$ ugustimes & Ph. Huget K.Hatest \\
\hline Samiago & Chile & Instituro de Radiomedicina & R.Arriagada, 1.Shwarcman \\
\hline Besancon & France & Uniwersity Hospial & O. Goubard, J. F.Bossed \\
\hline Bondeatix & France & Institur Bergonit & J-Mid Oilhugedy, S.Landriate \\
\hline Dijon & France & Centre G. PlLecterio & 1. Barillor, S.Naudy \\
\hline Grenoble & France & Wniwersity Hospinal & M.Bolla, A, Dussers \\
\hline Nice & France & Centre Antoine-Lacassagne & I.Lagrange, M.Petses \\
\hline Renowes & France & Centre Régional Eugetme Marquins & Gohenal \\
\hline Villejuif & France & Instintut Gustave Roussy & I. Ph. Pignol, H.Bouhnik \\
\hline Berlin & Germany & Universicy Hospital Charité & V.Budach, J Groll, U.Jahn \\
\hline Görningen & Germany & University Hospital & van Drunen \\
\hline Köln & Germany & Uniwersity Hospial & R. Bongatre \\
\hline Tubingen & Germany & Uniwersity Hospital & N. Weidner \\
\hline
\end{tabular}




\begin{tabular}{|c|c|c|c|}
\hline Harfin & listad & Rambam Medical Center & D. Barm Deromia \\
\hline Telowhoner & ferat & Clatur Shida Medical Center & M.Raphat Pfeffer, J.Goffmar \\
\hline Rombo & draly & Ospedale Sant Anra & M. Valli, AMonti, A Ostinelli \\
\hline Genowal & Waby & Ist. Naz per la riserca stal concro & MFoppiano, M.Guend \\
\hline Gudanist: & Boland & University Hospical & D. Jassem \\
\hline Bancelona & Spain & Insuturo Carala dOncologat & S. Yitlla, J Moreno \\
\hline Geneve & Switzerland & University Hospical & J. Kurm, Wh. Nouet \\
\hline Lauranne & Switzerland & University Hosputal & W. Jeannerec, H.Do \\
\hline Turich & Switserland & Univerisity Hospital & Ch.Ghanzmann, J.Dwis \\
\hline Amsterdam & The Netherlands & Nederlands Kanker Instítuat & B. Pieters, C. Hurkmans \\
\hline Deventer & The Newerlands & RTI Sredendriehoek a. & A.Mak, A.van 't Rier \\
\hline Enschede & The Netherlands & Medisch Spectrum Twente & I. Jobsen \\
\hline Groningen. & The Netherlands & Unuversity Hospital & W. Dolsma, Pvan der Hulst \\
\hline Tilburg & The Netherlands & Dr. Bernatd Vefbeten Instiruut & Ph. Poortmans, J Venselar \\
\hline Ularedht & The Netherlands: & University Medical Center & H.Struikmans, J Lagendijlk \\
\hline Istanbul & Turkey & University Hospital & M. Dincer \\
\hline Nowngham & Unired Kingdom & Notungham Cicy Hospical & D.Morgan, J.Berridge \\
\hline
\end{tabular}

Appendix A. List of participants in the dummy run.

\section{References}

1. Atkins $\mathrm{H}$, Hayward JL, Klugnman DJ, Wayte AB. Treatment for earlly breast cancer: a report after 10 years of a clinical trial. Br Med J 1972;2:423-429.

2. Bentel G, Marks LB, Hardenbergh P, et al. Variability of the locanion of internal mammary vessels and glandular breast tissue in breast cancer patients undergoing routine CT-based treatment planning. Int J Radiar Oncol Biol Phys 1999;44:1017-1025.

3. Bruce J. Operable breast cancer: a controlled clinical trial. Cancer 1957:28:1443-1452.

4. Cuzick J, Stewart H, Peto R, et al. Overwiew of randomised trials comparing radical mastecromy withour radiotherapy against simple mastectomy with radiotherapy in breast cancer. Cancer Treat Rep 1987;71:7-14.

5. Cuzick J. Stewart H, Rutquist J, et al. Cause-specific mortality in long-term-survivors of breast cancer who participated in trials of radiotherapy. J Clin Oncol 1994;12:447-453.

6. Ege GN, Clarck RM. Internal manmary lymphoscintigraphy in the conservarive surgical management of breast carcinoma, Clin Radiol 1980;31:559-563.

7. Fisher B, Slack NH, Cavanaugh PJ, et al. Postoperative radiotherapy in the treatment of breast cancer: results of the NSABP clinical trial. Ann Surg 1970;172:711-730.

8. Fisher B, Wickerham DL, Deutsch $\mathrm{M}$, $\mathrm{Cr}$ al. Breasr cumour recurrence following lumpectomy with and withour breast irradiation: an overview of recent $\mathrm{NSABP}^{3}$ findings. Semin Surg Oncol 1992;8:153-160.

9. Freedman GM, Fowble BL. Nicolaou $\mathrm{N}$, et al. Should internal mammary lymph nodes in breast cancer be a target for the radiation oncologist? Int 』 Radiar Oncol Biol Phys 2000;46:805-814.

10. Hamilron T, Langlands $A O$, Prescotr $\mathbb{R}$. The treatment of operable cancer of the breast: a clinical trial in the Southeast region of Scotand. Br J Surg 1974;61:758-761.

11. Handley RS. A surgeon's view of the spread of brease cancer. Cancer 1969;24:1231.

12. Harris JR, Hellman S. Put the "Hockey stick" on ice. Int J Radiat Oncol Biol Phys 1988;15:497-499.

13. Hurkmans CW, Saarnak AE, Pieters JH, er al. An improved technique for breast cancer irradiation including the loco-regional lymph nodes. Radiother Oncol 2000;55:145-151.

14. Hojris 1 , Overgatrd M, Christensen JJ, Overgaard J. Morbidity and mortality of ischemic heart disease in highrisk breast-cancer parients after adjuvant post-mastectomy systemic trearment with or without radiotherapy: analysis of DBCG $82 \mathrm{~B}$ and $82 \mathrm{C}$ randonised trials. Lancet 1999:354:1425-1430. 
15. Kane $S$, Johansen $H$. Breast cancer \pm a comparison of che results of simple mastecromy with postoperative roentgen irradiation by the Methriter method with those of extended radical mastectony. Acti Radiol 1959; 188(Suppl): 155-161.

16. Kaija H. Mauna P. Tangential breast irradiation with or withour internal manmary chain irradiation: results of a randomized trial. Radiother Oncol 1995:36:172-176.

17. Lacour J, Bucalossi P, Caceres $\mathrm{E}$, et al. Radical mastecromy wersus radical mastectomy plus internal nammary dissection. Cancer 1976;37:206-214.

18. Lagendijk JJ, Hofman $\mathbb{P}$. A standardized multifield irradiarion technique for breast numours using asymmetrical collimators and bean angulation. Br J Radiol 1992;65:56-62.

19. Lebesque JV. Field matching in breast irradiation: an exact solution to a geomerrical problem. Radiother Oncol $1986 ; 5: 47-57$.

20. Levitr $\mathrm{SH}$. Is there a role for post-operarive adjuvant radiation in breast canoer? Beautiful hypothesis versus ugly facts: 1987 Gilber H. Fletcher lecture. Int J Radiat Oncol Biol Phys 1988;14:787-796.

21. Overgaard $M$, Hansen PS, Overgaard J, et all. Postoperarive radiocherapy in high-risk premenopausal women with breast cancer who receive adjuvant chemotherapy. N Lingl J Med 1997;337:949-955.

22. Overgaard $\mathrm{M}$, Jensen $\mathrm{M}-\mathrm{J}$, Overgaard J, et al. Postoperative radiotherapy in high-risk postmenopatral breastcancer patients given adjuvant Tamoxifen: Danish Breast Cancer Cooperative Group DBCG 82 c randomised trial. Lancet $1999 ; 353: 1641-1648$.

23. Patterson R, Russell M. Clinical trials in malignant disease: part III. Breast cancer. Evaluation of postoperative radiotherapy. J Fac Radiol 1959;10:175-180.

24. Pierce LJ, Lichter AS. Postmastectomy radiotherapy: more than locoregional control. I Clin Oncol 1994:12:444446.

25. Poortmans $\mathrm{P}$, Venselaar I, Struikmans $\mathrm{H}$, et al. The potential impact of treatment variations in radiotherapy of the internal mammary lymph node chain: a quality assurance report on the dummy run of EORTC phase III randomised trial 22922/10925 in stage I-III breast cancer. Int J Radiat Oncol Biol Phys 2001 in press.

26. Ragaz J, Jackson SM, Le N, er al. Adjuvant radiotherapy and chemotherapy in node-positive premenopausal women with breast cancer. N Engl J Med 1997;337,956-962.

27. Recht $A$, Siddon RL, Kaplan WD, et al. Three-dimensional internal mammary lymphoscintography implications for radiation therapy treatment planning for breast cancer. Int J Radiat Oncol Biol Phys 1988; 14(3):477-481.

28. Romestaing $\mathrm{P}$, Ecochard $\mathrm{C}$, Hennequin $\mathrm{C}_{3} \mathrm{etal}$. The role of internal mammary chain irradiation on surwival after mastectomy for breast cancer \pm results of a phase III SFRO Trial (abstract 306). Proceedings of the 19th ESTRO Meeting, Istanbul, 2000.

29. Rutquist LE. Novel approaches using radiation therapics. Recent Results Cancen Res 1998:1522255-264.

30. Struikmans $\mathrm{H}$, wan Rijk PP. Optimizing radiotherapy of the internal mammary chain in breast carcinoma by scintigraphy. Radisother Oncol 1996,41:15-20.

31. Van den Bogaer W, Struikmans $H$, Fourquet $A$, et al. Internal mammary and medial supradivicular (IM MS) lymph node chain irradiation in stage I-III breast cancer. A phase III randomised trial of the EORTC, protocol 22922-10925, May 1996.

32. Van Limbergen $E$, Rijnders $A$ van der Schueren $E$, er al. Cosmetic evaluation of breast conscrving treament for mammary cancer. 2. A quantitarive analysis of the influence of radiarion dose, fractionarion schedules and surgical treatment techniques on cosmetic results. Radiother Oncol 1989;16:253-267.

33. Veronesi $U$, Vallegussa P. Inefficacy of incernal mammary node dissection in breast cancer surgery. Cancer $1981: 47: 170-175$. 


\footnotetext{
?

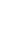

$\therefore$

.

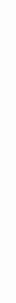

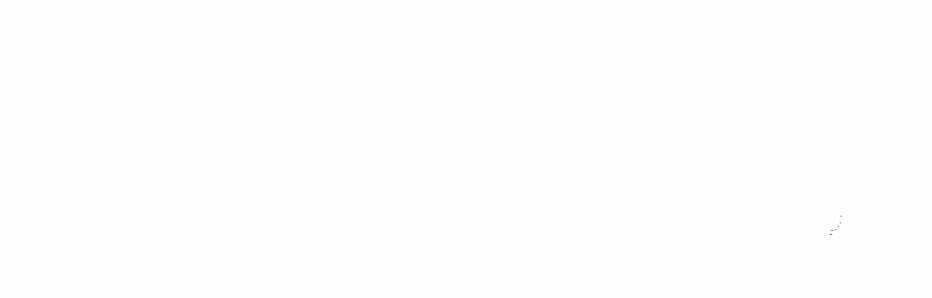




\section{CHAPTER 8}

Quality assurance of EORTC trial 22922/10925 investigating the role of internal mammary - medial supraclavicular irradiation in stage I-III breast cancer: the individual case review.

P. Poortmans, VE. Kouloulias, J.L. Venselaar, H. Struikmans, J.B. Davis, D. Huyskens, G. van Tienhoven, C. Hurkmans, B. Mijnheer, W. Van den Bogaert.

Eur J Cancer 39: 2035-2042, 2003. 


\begin{abstract}
To assess consistency among participants in an European Organisation for Research and Treatment of Cancer (EORTC) phase III trial randomising between irradiation and no irradiation of the internal mammary and medial supraclavicular (IM-MS) lymph nodes, all participating institutes were invited to send data from 3 patients in each arm as soon as they started accrual. The evaluation focused on eligibility, compliance with the radiotherapy guidelines, treatment techniques and dose prescription to the IM-MS region. Nineteen radiotherapy departments provided a total of 111 cases, all being eligible. Minor discrepancies were found in the surgery and pathology data in almost half the patients. Major radiotherapy protocol deviations were very limited: 2 cases of unwarranted irradiation of the supraclavicular region and a significant dose deviation to the internal mammary region in 5 patients. The most frequently observed minor protocol deviation was the absence of delineation of the target volumes in $80 \%$ of the patients. By detecting systematic protocol deviations in an early phase of the trial, recommendations made to all the participating institutes should improve the interinstitutional consistency and promote a high-quality treatment.
\end{abstract}

Keywords: Randomised clinical trial; Individual case review; Quality assurance; Breast cancer; Radiotherapy; Intemal mammary chain 


\section{Introduction}

In operable breast cancer ( $\mathrm{TO}-3, \mathrm{NO}-2, \mathrm{MO}$ ), the influence of regional lymph node involvement on prognosis is well known [1]. The incidence of lymph node invasion of the internal mammary chain ranges from $4 \%$ in axillary node-negative patients with upper lateral tumour location to $72 \%$ in axillary node-positive patients with lower medial tumour location [2-4]. In spite of this significant risk of tumoral involvement and several decades of a combination of surgery and radiotherapy in the local treatment of breast cancer, there remains an important controversy concerning the usefulness of irradiating the regional lymph node areas [5-12]. A meta-analysis of randomised trials of postoperative adjuvant radiotherapy, demonstrated a reduction of the long-term breast cancer death rate in the group of irradiated patients [13]. This was later confirmed in three randomised trials, which demonstrated the positive impact of adjuvant locoregional irradiation, including the internal mammary and supraclavicular lymph node areas, on survival in high-risk patients [14-16]. The explanation of the observed advantage remains unclear: the prevention of local recurrences through irradiation of the chest wall, the prevention of regional recurrences through irradiation of the lymph nodes, or both. To date, only one randomised radiotherapy trial on the effectiveness of adjuvant internal mammary irradiation alone has been published [17]. The follow-up is too short and the number of patients too limited (270) to provide an answer on the usefulness of adjuvant irradiation of the internal mammary chain. The thin line between the advantages and the side-effects of internal mammary and medial supraclavicular (IMMS) irradiation was very recently summarised in an editorial by Lievens and Van den Bogaert [18].

In May 1996, the Radiotherapy and the Breast Cancer Groups of the European Organisation for Research and Treatment of Cancer (EORTC) initiated a large phase III randomised multicentre trial. Its objective is to investigate whether adjuvant iradiation of the IM-MS lymph node chain for patients with localised breast cancer stage I-III with medially or centrally located tumours and/or axillary lymph node invasion after lumpectomy and axillary node dissection or modified radical mastectomy will significantly improve overall survival by at least $5 \%$ at 10 years [19]. Furthermore, the incidence of serious late side-effects on the heart and the lungs will be registered for patients treated with modern radiotherapy equipment and techniques for treatment preparation and treatment execution.

A large number of patients (3780) and a long follow-up (10 years) will be needed to answer the questions of this trial. To allow many radiotherapy institutes to participate in the trial and to accrue a large and representarive sample of patients, a standard treatment 
technique for the MM-MS irradiation is recommended and described in the protocol (Fig. 1). In this technique, the IM-MS lymph node area is treated with mixed photon and electron beams matched to the tangential field borders of the breast or thoracic wall (which can alternatively be treated with a direct electron field). Individual anatomical localisation of the internal mammary nodes was not mandatory for the standard technicque, but the use of a computed tomography (CT) scan, ultrasound or lymphoscintigraphy was recommended. This information can be used to select the most suitable electron beam energy, the dose prescription depth for the photon beam and the relative beam weights. In addition, the optimal beam dimensions depend on the location of the nodes. Several institutes have developed specific irradiation techniques for this indication [20]. These more complex treatment set-ups may be accepted in the trial, provided that they use individual localisation of the internal mammary nodes.

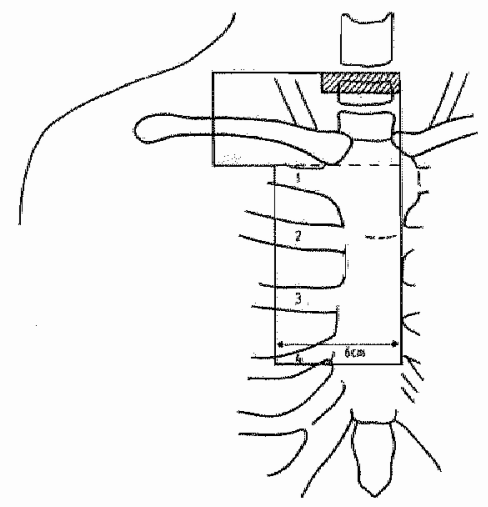

Figure 1. Recommended standard tectrique for internal mammary and medial supraclavicular irradiation $(M-M S)$

The Quality Assurance (QA) programme of the EORTC Radiotherapy Group aims to test the ability of the participating institutes to comply with the protocol guidelines. The vartiations in the dose delivered to the target volumes due to uncertainties in treatment planning systems (TPS) and in the calibration of the creatment machines were investigated by the general EORTC Radiotherapy Group QA procedures for institutional infrastructure. In the early phase of clinical trials, various QA procedures were performed to check protocol compliance and to detect and correct possible ambiguities in the protocol, which might introduce systematic protocol deviations [21-26]. In EORTC trial 22922/10925, the specific QA programme consists of (i) control of data consistency at the EORTC Data Centre by a double data entry procedure in the database, (ii) a dummy run procedure, (iii) an individual case review procedure (ICR) and (iv) mailed TLD electron dosimetry. The evaluation of the dummy run has already been reported, indicating a number of deviations in treatment set-up and dose prescription [27]. Based 
on the dummy run, a thorough evaluation of the irradiation techniques used in the 22922/10925 EORTC trial for the irradiation of the internal mammary and medial supraclavicular lymph node chain was also reported [20]. The external audit of electron beams using TLD dosimetry was performed by the EQUAL-ESTRO structure [28].

The current paper describes the outcome of the analysis of the individual case review from the 19 major participating institutes accruing $77 \%$ of the patients. The objective was to evaluate data consistency and to detect deviations from the protocol guidelines in a selection of patients accrued in the early phase of the trial.

\section{Patients and methods}

All participating institutes were invited at the onset of the trial to send six complete patients' records (3 patients with irradiation of the IM-MS and 3 without) including the surgical and pathology report, radiation treatment charts, treatment planning calculations and plots, as well as information on the primary tumour site and adjuvant systemic treatment. To facilitate and improve the evaluation, several case report forms (CRF) collected at the EORTC Data Centre were used. All data from the participating institutes were mailed to 'Tilburg where they were checked within weeks of receipt by the co-ordinating radiation oncologist. This allowed a quick detection of possible protocol deviations and enabled a prompt response to the participating institutes in the form of a letter and a copy of the Evaluation-CRF (E-CRF), developed and used for the evaluation of each individual patient (Table 1). In some cases, participants were asked to clarify or to complete the information supplied. At several of the bi-annual meetings of the EORTC Radiotherapy Group, an overview of the findings of the ICR was presented and discussed. Based on the results of the ICR, as well as of the dummy run, a letter with recommendations to improve compliance with the protocol was sent to all participants, including those who did not yet participate in the QA programme. Furthermore, a revised protocol was introduced in May 1999, including several improvements and clarifications based upon the findings of the QA procedures.

As displayed in Table 1, the evaluation focused on the eligibility to the protocol ( 8 items), surgery (breast and axilla) (3 items), pathology ( 4 items), radiorherapy (18 items) and systemic therapy ( 4 items). Some space for additional comments was allowed on the $\mathrm{E}$ CRF. All items were scored for all patients on the dedicated E-CRF and collected in a spreadsheet. Depending on the specific item, the score could be qualitative (yes, no, unknown) or quantitative (dose or number of days). For the evaluation of the calculated dose to the IM lymph node region, a reference point was defined at the cranial or central 
(whichever was the most relevant for the specific patient) treatment-planning slice through the breast target volume, representing an estimation of the anatomicall location of the IM lymph nodes. Results were analysed for the entire group of patients and also per participating centre and per treatment arm.

\begin{tabular}{|c|c|}
\hline \\
\hline \multicolumn{2}{|c|}{$\frac{\text { Eligibility }}{\text { Tlistologicalyoconfirmed invaiswe adenocarcinoma? }}$} \\
\hline & 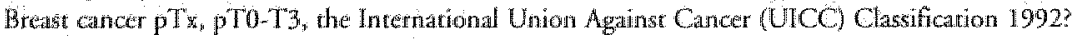 \\
\hline & Bralst cancer pNO-N2, UICC Classification 1992? \\
\hline & Abstince of distant metastaseng \\
\hline & Mastectomy or breast-conserwing suggery and axillary dissection? \\
\hline & Lethal/N1-2 or cencromedial/N0 1-2 rumour location? \\
\hline & Woman corm 75 years" \\
\hline & Written informed consern? \\
\hline
\end{tabular}

Surgery

Was surgery performed accoorling to prowocol?

Type of breast surgery on OSF acconding to pathology report?

Axillary sugery on $\mathrm{OSF}$,according to prithology report?

\section{Pathology}

Primary adenocarcinoma of the breist?

Tumout size and T-stage on OSF corresponds with parhology teport?

Number of positive lympla nodes on OSF corresponds with parthology report?

Number of examined lymph nodes on OSF corresponds with pathology report?

\section{Radiotherapy}

Dose specification according to ICRU Report 50 ?

Dose homogeneity acconding to ICRU Report 50 ?

IM-MS nodes reated according to vandomisarion arm?

Target volume interal mammany and medial supaclawicular (M-MS) nodes drawn on parient contour?

"Tafget wolume breast or thoracic wall drawn on patient concout?

IM-MS rechnique used... .

1M/point ind widenal anaromical information?

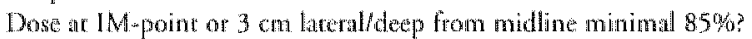

Dose 1 in $\mathrm{Cy}$ at $1 \mathrm{M}-$ point or $3 \mathrm{~cm}$ lareral/deep from midline. . .

IM.MS sccron energy conform protocol:

Dose spectication of MS nodes at 3 cm deptli?

IM-MS dose fracionation seheme $50 \mathrm{~Gy}$ in 25 fractons?

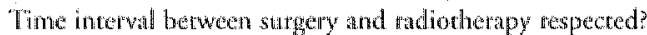

Number of days between date of surgery and forse fraction of radiotherapye...

Date of first irradiaton on OSF coincides with teatment dart?

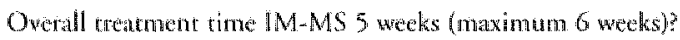

Overall tadiotherapy reatment vime in days...

Megavoltage film or alectronic portal imaging of IM-MS fedd?

\section{Systemic therapy}

Systemic therapy given oorresponds with OSF?

Type of systemic therapy: 1 whomonal therapy, 2 wechemotherapy, 3 moth

Intormation on dare of first cycle of chemotherapy?

Information on treatment scheme of chemotherapy?

OSFeron scudy form.

Table 1: Jtems sored on the wathation ase repon form tused for the individual case revien 
Treatment according to the other randomisation arm and over- or under dose at the IM point were defined as major protocol deviations. All other deviations were considered as being minor deviations, including the absence of delineation of target volumes because the advised standard treatment technique did not oblige the use of individual anatomical information.

\section{Results}

By January 2003, over 3300 patients were accrued by 45 institutes in 12 different countries. Nineteen radiotherapy departments, responsible for the accrual of $77 \%$ of the patients, participated in this ICR (Table 2). Eighteen out of those 19 institutes sent 3 cases per randomisation arm (six records per institute), while one institute sent only 3 cases ( 1 case without IM-MS irradiation, and 2 cases with IM-MS irradiation). Therefore, a total of 111 cases was available for evaluation, 55 cases in arm 1 and 56 cases in arm 2.

\begin{tabular}{|c|c|c|c|}
\hline City & Country & Institution & $\begin{array}{l}\text { Local responsible radiation oncologist } \\
\text { and physicist }\end{array}$ \\
\hline Braxelles & Belgium & Universily Hospital Saint-Luc & C. Kinkhove, S. Wynckier \\
\hline Leuwen & Belgium & University Hospital & W. wan den Bogaert. D. Huyskens \\
\hline Santiago & Chile & Insticuro de Radiomedicina & R. Arriagadal, L, Schnwarcoman \\
\hline Bordeaux & France & Instirut Bergonié & J.-M. Dilhuydy, V. Luric \\
\hline Dijon & France & Centre G.-FLederc & 1. Barillot, S. Naddy \\
\hline Grenoble & France & University Hospiral & M. Bolla, A.n Dusserre \\
\hline Pats & France & Instivat Curie & A. Fourquer \\
\hline Willejuif & France & Institur Gustave Roussy & C. Le Pechotik \\
\hline Berlin & Getmany & Universiry Hospiral Charite & V. Bubach, U. Whan, J. Gooll \\
\hline Koln & Germany & University Hospicall & R. Bongarty \\
\hline Tubingen & Germany & University Hospiral & M. Bamberg, N. Weidner, E. Hermann \\
\hline Como & Irally & Ospedate Sant'Anna & M. Vall, An Musti, A. Ostinelli \\
\hline Genève & Swizerland & Universtry Hospital & 1. Kurc, Th. Nourt \\
\hline lausarne & Swilzerland & University Hospital & W Jeannere, H. Do \\
\hline Zürich & Switzerland & Uniwersity Haspital & Ch. Glanamann, J.B. Davis \\
\hline Amscerdam & The Necherlands & Nederlands Kanker Instritutut & B. Meters, C, Hurkmans \\
\hline Deventer & The Netherlands & RTI Stedendriehoek co. & I. limmertech $A$, wan $\mathrm{C}$ Rict \\
\hline Tilburg & The Nerlietlands & Dr. Bernard Verbeten Insticuut & Ph. Poormmans, I. Vensentiar \\
\hline Uredr & The Nethetands & University Modical Centre & H. Sruikmanas J. Eagendijk. \\
\hline
\end{tabular}

EORTC, Europenn Organisation for Reasearch and Treament of Cancen:

Table 2: List of participants in the quality assumance programme of EORTC trial 


\subsection{Eligibility}

All 111 patients were fully eligible, without any item being incomplete. 88 patients $(79 \%)$ were treated with breast-conserving techniques. Only 23 of the 111 patients $(21 \%)$ were treated with a modified radical mastectomy.

\subsection{Surgery}

In 1 patient, the type of breast surgery reported was not in agreement with the pathology report: on the CRF, a mastectomy was reported whereas, according to the pathology file and the other patient and treatment data, it was clear that a tumorectomy and a reexcision had been done. In another patient, some items were not correctly specified on the CRF, one pathology report was missing and in 2 patients some of the surgical details were not available. In 15 out of the 19 centres (79\%), all data were present and correctly reported.

\subsection{Pathology}

The tumour size, as mentioned in the pathology report, was not correctly reported on the CRF in 14 patients (13\%) and could not be evaluated in 8 other cases (7\%). The total number of tumour positive or of examined lymph nodes was not correctly reported in 1 and 5 patients, respectively, and could not be evaluated in 7 cases (6\%) each. Supplementary remarks were: incorrectly specified items on the CRF in 17 patients $(15 \%)$, there were missing pathology reports and missing CRF in 6 patients each $(5 \%)$. In 1 case, immediate breast reconstruction with prosthesis was performed, but not reported. Only six centres (32\%) complied completely for all their patients.

\subsection{Radiotherapy}

2 patients, randomised to arm 1, were not irradiated at all because of a lack of indication for post-mastectomy irradiation of the thoracic wall. For the same reason, 2 other parients, randomised to arm 2, were only treated in the IM-MS lymph node region. 2 parients, randomised to arm 1 , were erroneously irradiated at the supraclavicular lymph node region.

Dose specification was not according to the International Commission on Radiation Units and Measurements (ICRU) Report 50 [29,30] in 10 cases (9\%). Dose homogeneity was not within the prescribed limits for 1 patient and could not be evaluated for 2 patients. In 4 cases (7\%), the fractionation schedule of IM-MS irradiation differed from the prescription of 25 daily fractions of 2 Gy (24-28 fractions of $1.8-2.25$ Gy). The target volumes for the breast/thoracic wall and for the IM-MS lymph nodes were not drawn on the treatment plans in $87(80 \%)$ and $64(59 \%)$ of the patients, 
respecrively. However, 95 patients $(87 \%)$ had an individual localisation of the IM-MS nodes, either on CT scans ( 83 patients) or using ultrasonography, lymphoscintigraphy or combinations. In the 56 cases of irradiation of the IM-MS, most centres used the standard treatment technique as described in the study protocol (14 centres (74\%) with 41 cases). However, for 32 of these 41 cases $(78 \%)$, anatomical information was used to individualise the field set-up. Four centres $(21 \%)$ with 12 patients developed an individualised treatment set-up. In one centre with 3 patients, the choice between the standard technique or a customised technique depended on the individual parient's anatomy. The specified maximum interval of 8 weeks between surgery and randomisation, unless chemotherapy was given in between, was not met in 10 cases (9\%) from six centres. The overall treatment time of the IM-MS lymph nodes ranged from 25 to 55 days (mean=38.3 \pm 5.5$)$ : less than 6 weeks in $48(86 \%)$ and less than 7 weeks in all 56 cases. In 22 cases (39\%), information about portal imaging of the IM-MS field was missing. In all centres, at least one item on radiotherapy was missing or incompletely reported.

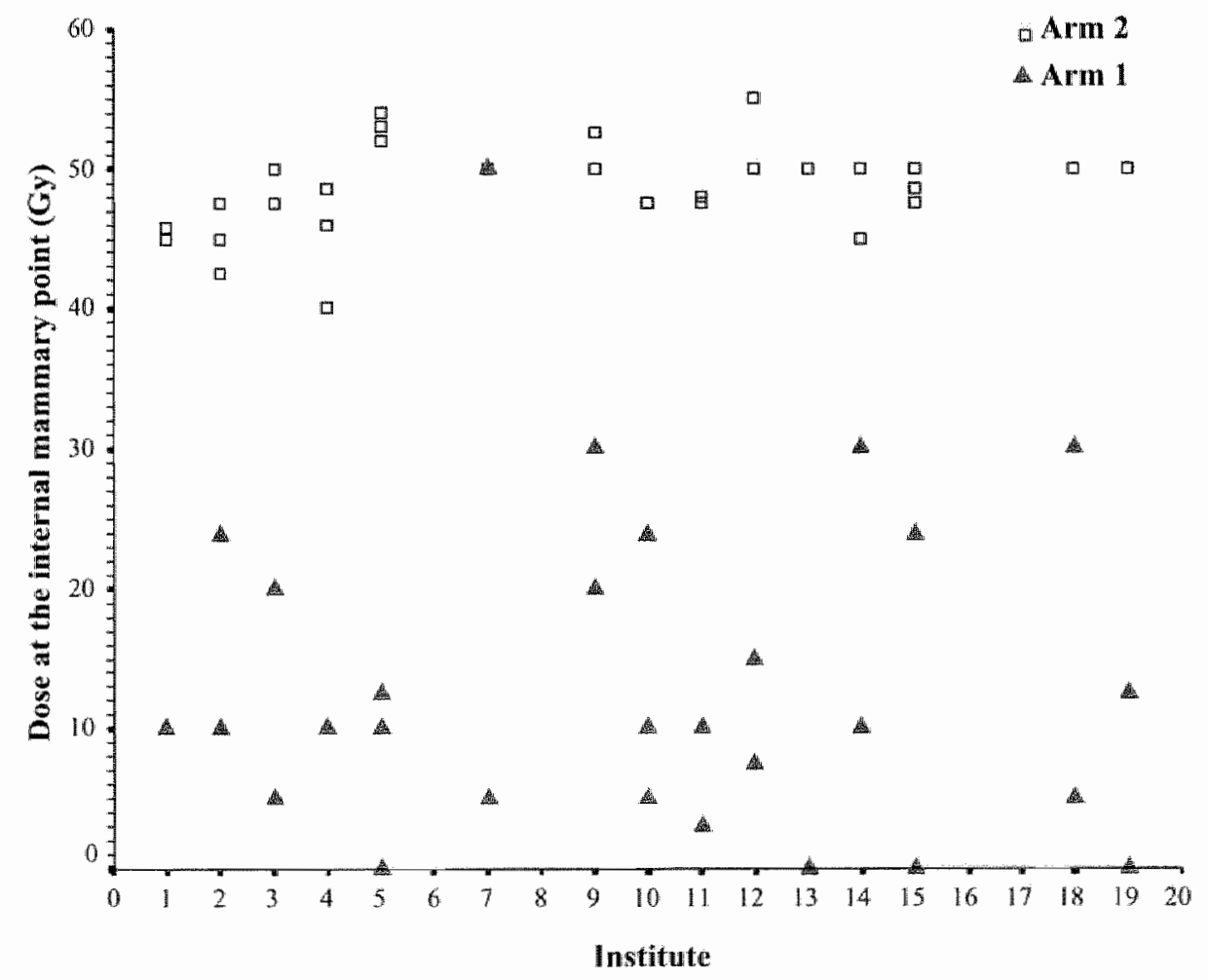

Figure 2. Dose at the internal mammary point stratified by institute and per randomisation arrw. The wean dose ( 1 standard deviation) in arm 1 was $12.8 \pm 11.7$ Gray (Gy) and in arm 2, 48.7.3.3 Gy. 


\subsection{Dose to the IM-MS lymph node region}

In the 56 cases of irradiation of the IM-MS, we had sufficient information to recalculate the dose to the IM lymph node area in 45 patients $(80 \%)$. The median dose in this group was $49.25 \mathrm{~Gy}$. It was at least $85 \%$ of the prescribed dose of $50 \mathrm{~Gy}$ in 44 patients (98\%). In 37 cases (66\%), the dose to the MS lymph nodes was prescribed at the 3-cm depth as specified in the protocol, in 17 cases $(30 \%)$ it was prescribed at a different depth (in general at the depth of Dmax) and in 2 cases (4\%) it was not retrievable. In the 55 patients randomised to arm 1, i.e. without irradiation of the IM-MS, the dose to the IM lymph node area could be recalculated in 42 patients $(76 \%)$. The median dose here was 9 Gy. For 9 patients (21\%), this dose was more than $25 \%$ of $50 \mathrm{~Gy}$ and for 4 patients $(10 \%)$ even in excess of $50 \%$. The dose to the IM lymph node area is displayed in Fig. 2.

\subsection{Systemic therapy}

Guidelines on systemic therapy were not included in this protocol. However, institutes had to specify their general policies concerning adjuvant treatment. All data were present and correctly reported for all patients in nine centres (47\%). Information on adjuvant systemic treatment was not complete for 13 patients (12\%). The treatment given according to the patient file did not correspond to the CRF in 7 patients (7\%). 27 patients (24\%) received chemotherapy only, 32 (29\%) hormonal therapy only, and 23 (21\%) a combination of hormonal and chemotherapy. Only 20 patients (18\%) did not receive any adjuvant systemic treatment.

\section{Discussion}

EORTC 22922-10925 trial investigates the impact of elective irradiation of the internal mammary and medial supraclavicular lymph node chain on long-term survival for patients with localised breast cancer stage I-III with medially or centrally located tumours and/or axillary lymph node invasion after lumpectomy and axillary node dissection or modified radical mastectomy. To guarantee the accrual of a large number of patients within an acceptable period of time, the participation of a large number of radiotherapy departments is necessary. Therefore, treatment set-up guidelines in the protocol were not very strictly defined. A simple treatment technique was suggested, but more complex treatment set-up techniques were also allowed. At the time of activation of the protocol, a QA committee was formed in order to initiate the necessary procedures to guarantee the quality of the data by limiting the inter-institutional variability and by detecting potential systematic protocol deviations which may be due to possible ambiguities in the protocol guidelines. The dummy run procedure has already shown a significant number 
of minor deviations from protocol guidelines [27]. In this paper, the results from the individual case review procedure are described. Both eligibility as well as protocol compliance was evaluated for 3 patients randomised to each of the two treatment arms.

\subsection{Eligibility}

All 111 patients were eligible for participation in this trial according to the inclusion and exclusion criteria defined in the protocol. Concerning the details on surgery, only one item in 1 patient was not correctly specified. In 4 patients, less important deviations or missing information were noted. For pathology data, a total of 20 deviations in 18 patients was found. One centre forgot to send evaluation data on this item for all their patients, with missing data on four items in a further 3 patients. We conclude, therefore, that compliance to the eligibility criteria of the trial and participation to the QA programme was excellent in the majority of the patients of the 19 centres who participated in the ICR. The 26 centres that did not participate included only $23 \%$ of all randomised patients. Nevertheless, their participation to the QA should be encouraged to cover as much as possible the smaller centres with less experience in the participation of clinical trials. Remarkably, all patients from seven of the centres were treated with a breast-conserving approach, which might suggest a kind of patient selection by those participants.

\subsection{Protocol compliance}

The number of major protocol deviations encountered was very limited. In the early phase of the trial, 2 patients randomised to arm 1 received radiotherapy to the supraclavicular area. After having highlighted this point during meetings of the EORTC radiotherapy group and in the list of recommendations sent to all participants, this was not encountered again. The dose calculated at the IM point was in the vast majority of the cases according to the randomisation arm: at least $85 \%$ of the prescribed dose in all but 1 of the patients randomised to arm 2 and in only 4 patients randomised to arm 1 , was it more than $50 \%$ of the prescribed dose of $50 \mathrm{~Gy}$ in 25 fractions. Depending on the individual patient's anatomy, but especially when the thoracic wall was treated with one or more electron beams, it was difficult to avoid giving part of the dose also to the IM lymph node area for patients randomised into arm 1 . Suggestions made to the participants to decrease the dose at the IM point included an adaptation of the field setup and the local use of tissue equivalent material.

Only three of the participating centres irradiated the IM-MS lymph node region without the use of individual anatomical information. They all followed strictly the standard treatment set-up technique as described in the trial guidelines. Centres using customised 
anatomical localisation of the internal mammary nodes were encouraged to adapt the electron beam energy, the prescription depth for the photon beam and the relative beam weights according to the patient's anatomy when using the standard technique. Five of the participating institutes used more complex and highly individualised treatment setup techniques developed specifically for this indication.

A rather typical feature when using the standard technique for IM-MS irradiation together with breast/chest wall irradiation is the matching of the field junctions. Most of the institutes using the standard technique individualise the choice of an exact match or an overlap based on patient- and tumour-related characteristics. A modification of the standard technique was used in 11 cases, resulting in an improved dose distribution at the field junction [20]. Remarkably, only a minority of the participants clearly delineated the target volumes. Even with the actual CT-based patient anatomy clearly visible on the screen of the TPS, the absence of delineated target volumes might hamper proper treatment prescription and evaluation. Therefore, when writing future protocols, more attention should be paid to the proper definition and delineation of target volumes. The simplest way might be to recommend a strict adherence to the ICRU guidelines, although discrepancies will always occur because of the different interpretation of the clinical data by different clinicians.

In this individual case review, a high compliance to the patient-inclusion criteria was found. A number of potentially systematic deviations from the protocol or from general guidelines, which might lead to a false-negative result of the end point, were nevertheless detected. This may indicate that even in protocols where, for reasons of accrual, the radiotherapy guidelines are kept as simple as possible, a more precise prescription of the procedures may be necessary.

Only 19 of the 45 participating institutes completed the ICR. However, since the 19 participants are the ones with the highest accrual (77\%), this can be judged as being of less importance. Moreover, by providing recommendations regularly to all participants, the ICR will have worked (like the dummy run) as oil spreading on the water. Both QA procedures were performed in the early phase of the trial, before a significant number of patients was accrued. This enabled the study coordinators and the QA committee to give recommendations for improvements to the participants individually as well as during the meetings of the EORTC Radiotherapy Group. A summary of all recommendations was soon sent to all participants and already in May 1999, less than 3 years after the start of the trial and before the accrual of one-third of the parients needed, a revision of the trial protocol was written and distributed. By doing so, participating institutes, including those which had not yet participated to the QA programme or did not actually start 
patient accrual, were encouraged to improve the compliance to the trial protocol and to refine the radiotherapy techniques used. This should lead to an improvement of the dose distribution of the radiotherapy of the IM-MS and to a decrease in the inter-institutional dose variarion, thereby strengthening the reliability of the final trial results.

\section{Acknowledgements}

The authors want to thank all persons involved at the participating centres as well as Marianne Pierart, data manager at the EORTC Data Centre.

\section{References}

1. Fisher $B$, Bauer $M$, Wickerham DL, et al. Relation of number of prositive axillary nodes to the prognosis of patients with prinnary breast cancer: a NSABP update. Cancer 1983,52,1551-1557.

2. Lacour J, Bucalossi P, Caceres $\mathbb{E}$, et al. Radical mastectony versus mdical mastectomy phus internal mammary dissection. Cancer 1976, 37, 206-214.

3. Levit SH. Is there a role for post-operative adjuvant radiation in breast cancer? Beauriful hypothesis versus ugly facts: 1987 Gilbert H. Fletcher lecture. Inx J Radiat Oncol Biol Phys 1988, 14, 787-796.

4. Veronesi U, Marubini E, Mariani L, et al. The dissection of internal mammary nodes does not improve the survival of breast cancer patients, 30-year results of a randonised trial. Eur J Cancer 1999, 35, 1320-1325.

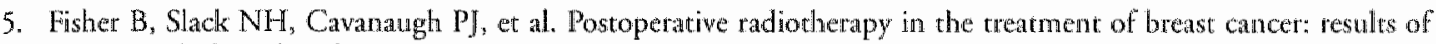
the NSABP clinical triad. Ann Surg 1970, 172,711-730.

6. Fowble B, Hanlon A, Freedman $G$, et all. Internal mammary node itradiation neirher decreases distant metastalses nor improves survival in stage I and II breast cancer. Int J Radiat Oncol Biol Phys 2000, 47, 883-894.

7. Cuziclk J, Stewart H, Peto R, et al. Overwiew of randomized trials of postoperative adjuvant radionherapy in breast cancer. Cancer Treat Rep 1987, 71, 15-25.

8. Harris JR, Hellman S. Put the "Hockey stick" on ice. Int J Radiat Oncol Biol Phys 1988, 15, 497-499.

9. Edland RB. Presidential address: does adjukant radiotherapy have a rolle in the postmilstectomy maningement of patients with operable breast cancer-revisited. Int J Radiat Oncol Biol Phys 1988, 15, 519-535.

10. Hetcher GH, Monague ED. Does adequate irradiation of the internal mam mary chain and supraclawicular nodes improve survival rates? Int J Radiat Oncol Biol Phys 1978, 4, 481-492.

11. Hurkmans $\mathrm{CW}$, Borger JH, Bos L,J, et al. Cardiac and lung complication probabilities afrer breast cancer irradiation. Radiother Oncol 2000, 55, 145-151.

12. Rurquist LE, Lax I, Fornander T, et al. Cardiovascular mortality in an randomized trial of adjuxam radianion therapy versus surgery alone in primary breast cancer. Int I Radiat Oncol Biol Phys 1992, 22, 887-896.

13. Cuzick J. Srewart H, Rutquist LE, et al. Cause-specific mortality in long-term survivors of breast cancer who participated in trials of radiotherapy. I Clin Oncol 1994.12, 447-453.

14. Overgaard $M$, Hansen PS, Overgaard J, et al. Postoperarive radiorherapy in high-risk premenopausal women with breast cancer who receive adjuvant chemotherapy. N Engl I Med 1997, 337, 949-955.

15. Overgaard M, Jensen M-J, Overgaard J, et al. Postoperative radiotherapy in high-risk posmenopausal breastancer patients given adjuvant tamoxifen: Danish Breast Gancer Cooperative Group DBCG 82c randomised trial. Lancet $1999,353,1641-1648$.

16. Ragaz J, Jackson SM, Le $\mathrm{N}$, et al. Adjuvant radiotherapy and chemotherapy in node-positive premenopausal women with breast cancer. N Engl J Med 1997, 337, 956-962.

17. Kaija $\mathrm{H}$, Mauna P: Tangential breast irradiation with or without internal mammary chain irradiation: results of a randomized trial. Radiother Oncol 1995, 36, 172-176.

18. Lievens $Y$, Van den Bogaert W. Inernal mammary and medial supradavicular lymph node irradiation: the thin line berween adwantages and side effects [edicorial]. Radiother Oncol 2002, 55, $75-77$. 


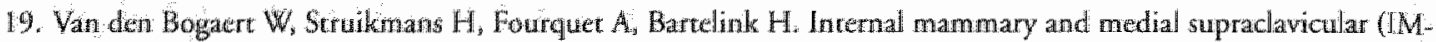
MS) Iymph node chain irradiation in stage I-III breast cancer. A phase III randomised trial of the EORTC, protocol 22222/10925, May 1996, rewision May 1999.

20. Lievens $Y_{\text {; }}$ Poormans D, Van den Bogaert W. A glance on quality assurance in EORTC study 22922 evaluating technicues for internal mammary and medial supraclavicular lymph node chain irradiation in breast cancer. Radiother Oncol 2001, 60, 257-625.

21. Kouloulias $V$, Poormans $P$, Bernier $\mathrm{J}$, et al. The quality assurance programme of the Radiocherapy Group of the European Organixation for Research and. Treament of Cancer (EORTC): a cricical appraisal of 20 years of continuous efforts. Euir] Cancer [in press]

22. Bolla M, Bartelink $H$, Garavaglia $G$, er al. EORTC guidelines for writing protocols for clinical trials of radiotherapy: Radiother Oncol 1995, 36, 1-8.

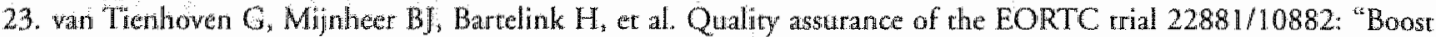
versus no boost in breast conserving therapy: an owerwiew". Surahlenther Onkol 1997, 173, 201-207.

24. Valley J-F, Bernier J, Tercier P-A, et al. Quality assurance of the EORTC radiatherapy trial 22931 for head and neck carcinomas: the dummy run. Radiother Oncol 1998, 47, 37-44.

25. Kouloulias V, Bosset JF, van Tienhoven $G$, Davis BJ, Pierart M, Poortmans P. Quality assurance in the EORTC 22921 trial on preperative radiotherapy with or withour chemorherapy for resectable rectal cancer: evaluation of the individual case review procedure. Bur J Cancer 2002, 38, $1849-1856$.

26. Thwates D. Quality atssurance into the next century [editorial]. Radiocher Oncol 2000, 54, vii-ix.

27. Poortmans $P M$, Venselaar $J \mathrm{~L}$, Struikmans $H$, er al. The potential impact of treatment vartations on the results of radiotherapy of the intemal mammary lymph node chain: a quality-assurance report on the dummy run of EORTC phase III randomized trial $22922 / 10925$ in stage I-III breast cancer(1). Int I Radiat Oncol Biol Phys $2001,49,1399-1408$.

28. Gomola I, van Dam J, Isern-Verdum J, et al. Exrernal audits of electron beams using mailed TLD dosimetry: preliminary results. Radiother Oncol 2001, 58, 163-168.

29. ICRU Report 50. Prescribing, Recording and Reporting Photon Beam Therapy. 7910 Woodmont Avenue, Bethesda, MD, 20814, USA. International Commission on Radiation Units and Measurements.

30. ICRU Report 62. Prescribing, Recording and Reporting Photon Beam Therapy (Supplement to ICRU Report 50). 7910 Woodmont Avente, Bethesda, MD 20814, USA, International Commission on Radiation Units and Measurements. 


\section{CHAPTER 9}

Quality assurance in the EORTC randomised trial 22922/10925 investigating the role of irradiation of the internal mammary and medial supraclavicular lymph node chain works.

Philip Poortmans, Philippe Lambin, Vassilis Kouloulias, Laurence Collette, Geertjan van Tienhoven, Henk Struikmans, Jack L.M. Venselaar, Walter Van den Bogaert, J. Bernard Davis, on behalf of the EORTC Radiotherapy and Breast Cancer Groups.

Eur J Cancer: submitted, 2005. 


\section{Abstract}

Purpose: The results of a dummy run and of an individual case review performed in the EORTC trial investigating the role of adjuvant internal mammary and medial supraclavicular irradiation in breast cancer are compared to evaluate the possible influence of recommendations on protocol compliance.

Materials and Methods: Prior to protocol activation participating institutes produced treatment plans according to the guidelines of the protocol based on manual outlines of an average patient. Thereafter they were asked to provide data on the first six randomised patients.

Results: The dummy run particularly yielded information on specific treatment techniques. The indiviclual case review collected additional patient- and tumour-related data, showing the use of anatomical information for treatment planning. A comparison between both procedures revealed that the treatment of real trial patients concurred more accurately with protocol guidelines than the dummy run.

Conclusions: This quality assurance program positively influenced the protocol compliance in this trial.

Keywords: Randomised clinical trial, Quality assurance, Breast cancer, Radiotherapy, Internal mammary chain. 


\section{Introduction}

In operable breast cancer, the role of regional radiotherapy remains a controversial issue. Several meta-analyses including 3 randomised trials have demonstrated a reduction of long-term breast cancer death rate in high-risk patients irradiated loco-regionally. This was partially counterbalanced by a relative increase of non-breast cancer related deaths, especially of vascular origin and exclusively seen in older trials using outdated radiotherapy techniques $[1,2,3,4,5,6,7,8]$. To date, only one randomised radiotherapy trial specifically dealing with the effectiveness of adjuvant internal mammary irradiation has been published [9]. The follow-up is too short and the number of patients too limited (270) to answer the question of the value of internal mammary irradiation. In a phase II prospective trial on high dose chemotherapy in high-risk breast cancer patients, some of the patients did not receive internal mammary irradiation due to technical reasons. These patients had a significantly decreased disease free and overall survival rate due to this omission [10]. From May 1996 to January 2004, the Radiotherapy and the Breast Cancer Groups of the European Organisation for Research and Treatment of Cancer (EORTC) conducted a phase III randomised multi-center trial to investigate the thin line between advantages and side effects of adjuvant irradiation of the internal mammary and medial supraclavicular (IM-MS) lymph node chain. Eligible patients had localised breast cancer stage I-III with medially or centrally located tumours and/or axillary lymph node invasion [11]. The protocol was approved by the ethics committees of all participating institutions and all patients signed an informed consent. A large number of patients and a long follow up period are needed to definitely answer the question whether elective irradiation of the IM-MS chain is of dinical benefit. To allow broad participation of radiotherapy institutes and to accrue a large and representative sample of patients, a socalled standard treatment set-up technique for the IM-MS irradiation was recommended and described in the protocol. More complex treatment set-ups were also accepted in the trial, provided that these were in accordance with the basic recommendations $[12,13]$.

It is well recognised that deviations from protocol guidelines can have an adverse effect on the results of the trial. Addicionally, the lack of evidence that a trial has been performed under strict conditions of quality assurance (QA) will reduce general acceptance of the results from such a trial. For these reasons and for several years now, the EORTC Radiotherapy Group has developed a large number of QA procedures for clinical trials to test the ability of the participating institutes to cope with protocol guidelines $[14,15,16]$. The variations in the dose delivered due to uncertainties in treatment planning systems (TPS) and in the calibration of the treatment machines are investigated by the general EORTC Radiotherapy Group QA procedures for institutional 
infrastructure with an independent beam output check. In the early phase of the accrual of clinical trials various QA procedures are performed to verify protocol compliance and to detect and correct possible ambiguities in the protocol, which might, if not corrected, lead to systematic protocol deviations. In EORTC trial 22922/10925 the specific QA program consisted of (i) control of data consistency at the EORTC Data Center by a double data entry procedure in the database, (ii) a dummy run procedure (DR) on "paper patients", (iii) an individual case review (ICR), and (iv) mailed TLD electron dosimetry. All these separate aspects of the QA have been reported earlier $[12,13,17,18]$.

This paper describes the comparison between the results of the DR and of the ICR. The degree of agreement between the information on different treatment parameters collected before the onset of the trial with the DR on paper patients and in the early phase of trial participation on real patients is discussed, including its' potential influence on the outcome of the trial.

\section{Materials and Methods}

For the DR, participants were asked to make a treatment plan for an anonymous and suitable patient after mastectomy and for another after lumpectomy, according to each of the 2 arms of the protocol [13]. After activation of the trial, a full set of data including patient, clinical and treatment related parameters for the first 3 patients randomised to each of the 2 treatment arms had to be collected for the ICR [18].

All data from the participating institutes were mailed to Tilburg for evaluation. This enabled a prompt response to the participating institutes in the form of a letter. In many cases, participants were asked to clarify or to complete the information supplied. At regular time intervals, a QA committee meeting was organised to discuss the findings and to complete the provisional answers already sent to the participating institutes.

The location of the IM-MS lymph nodes varies considerably [19,20]. To cover the target volume with at least the $95 \%$ isodose line for photons and $85 \%$ for electrons, the prescription point for photons is defined at $3 \mathrm{~cm}$ depth and the electron energy should be between 12 and $14 \mathrm{MeV}$ [21,22]. In the case of individual localisation of the IM-MS, participants were encouraged to adapt dose prescription, beam energy and relative weight of photons versus electrons to the individual patients' anatomy. For the evaluation of the calculated dose at the IM lymph nodes, 2 reference points were defined: the point P(IM) represents an estimation of the anatomical location of the IM lymph nodes and the point $P(3,3)$ is the point located $3 \mathrm{~cm}$ below the skin and $3 \mathrm{~cm}$ homolateral of the midline of the parient (Figure 1). The calculated dose at both points was measured on the central 
slice and on the cranial slice for the treatment plans according to both arms of the protocol, i.e. without and with IM-MS irradiation.

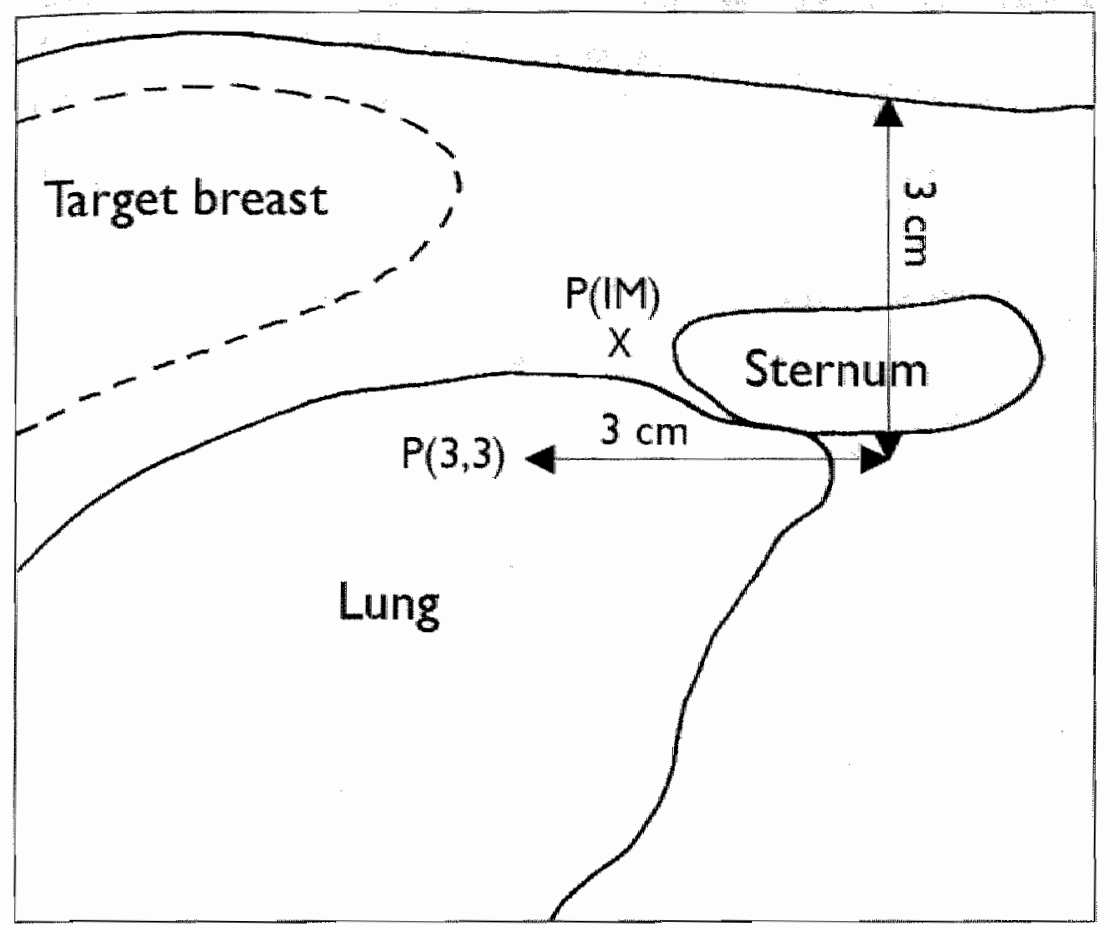

Figure 1: Illustration of the definition of points $P(I M)$ and $P(3,3)$.

The primary end-point of the trial is overall survival. The statistical considerations of the trial are based on an expected survival rate in the control group (without IM-MS irradiation) of $75 \%$ at 10 years. The minimal benefit considered to be of clinical relevance is a $4 \%$ gain in 10 years survival up to $79 \%$ at 10 years in the patient group with IM-MS irradiation. The first analysis will be carried out at the 0.022 significance level whenever 1000 deaths will have been reported. This number should provide $80 \%$ power to detect a real difference of $4 \%$. To estimate the theoretical influence of interinstitutional dose variation to the IM-MS lymph node chain on the outcome of the trial, we assumed, based on the typical sigmoid cell survival curves, that $0-49 \%$ of the prescribed dose to the IM-MS region has no effect, a dose of $50-84 \%$ has only $10 \%$ of the desired effect and a dose of higher than $85 \%$ has the full effect on survival probability [13]. 


\section{Results}

Between July 1996 and January 2004, 4004 patients had been entered in the study by 45 institutes in 12 different countries. The DR procedure was completed by 41 institutes, who accrued $93 \%$ of the patients. Twenty radiotherapy departments, responsible for the accrual of $76 \%$ of the patients, participated in the ICR. For 19 different participating institutes, accruing 73\% of the patients, the DR as well as the ICR were available and found suitable for comparison (Table 1).

\begin{tabular}{|c|c|c|c|}
\hline City & Country & Institution & $\begin{array}{l}\text { Local responsible radiation oncologist } \\
\text { and physicist }\end{array}$ \\
\hline Bruxelles & Belgitum & University Hospieal Saint-Luc & C. Kirkhove, S. Vynckier \\
\hline Leuven & Belgium & University Hospizal & A.Maes, W.van den Bogaer, D.Huyskens \\
\hline Santiago & Chile & Instinto de Radiomedicina & R.Arriagada, L.Shwartzman \\
\hline Bordenux & France & Institut Bergonié & J.-M.Dilhuydy, V.Lurie \\
\hline Dijon & France & Centre G.-F.Lederc & I.Barillot, S.Naudy \\
\hline Grenoblle & France & University Hospital & M.Bolla, A.Dusserre \\
\hline Villejuif & France & Institut Gustave Roussy & C.Le Pechoux, J.Ph. Pignol, H. Bouhnik \\
\hline Betlin & Germany & University Hospital Charite & V.Budach, U.Jahn, L.Schlenger, J.Groll \\
\hline Berlin & Germany & Robert-Rössle-Klinik & S.Koswig, H.Koppe \\
\hline Kölin & Germany & Universiry Hospital & R.Bongattrz \\
\hline Tiibingen & Germany & University Hospital & M,Bamberg, N.Weidner, E.Herrmann \\
\hline Como & Italy & Ospectale Sant'Anna & M. Valli, A.Monci, A.Ostinelli \\
\hline Genève & Switzerland & University Hospital & J.Kurk, Ph. Nouer \\
\hline Lausanne & Switzerland & University Hospital & W.Jeanneret, H.Do \\
\hline Zuirich & Swirzerland & University Hospiral & Ch.Glanzmann ${ }_{v}$ J.B.Davis \\
\hline Amsterdam & The Netherlands & Nederlands Kanker Institurut & B. Pieters $_{x}$ C.Hurkmans \\
\hline Deventus: & The Netherlands & RTI Stederidriehoek e.o. & A.Mak (†), A.van "t Riet \\
\hline Tillourg & The Netherlands & Dr. Bernard Verbeeten Instituut & Ph.Poortmans, I. Venselatar \\
\hline Urecht & The Netherlatids & Universty Medical Center & H.Struikmans, I.Lagendijk \\
\hline
\end{tabular}

Table 1: List of the 19 institutes who completed boch the dummy run and the individual case review.

The DR procedure was designed to evaluate the treatment techniques used by the different institutes and their compliance to the protocol guidelines, as well as to detect possible ambiguities in the trial protocol. In the ICR, several patient, tumour and treatment related variables were evaluated also to verify patients' eligibility. For the comparison between the DR and the ICR, we focussed on the treatment techniques and the calculated dose at the IM lymph node region.

\subsection{Treatment techniques:}

Irradiation of the breast alone was always performed by 2 tangential photon fields. In the DR, the irradiation of the thoracic wall after mastectomy was done in 12 institutes by 2 
tangential photon fields, in 5 institutes by a direct electron field and in 2 institutes no data were given on irradiation of the thoracic wall after mastectomy. In the $1 \mathrm{CR}, 13$ institutes used two tangential photon fields, 2 institutes a direct electron field and for 4 institutes data were missing.

For irradiation of the IM-MS lymph nodes, fifteen institutes in the DR and 14 in the ICR used the standard rechnique as described in the protocol with a direct mixed electron and photon beam to the IM-MS lymph node area, adjusted to the irradiation beans of the breast/thoracic wall. Four institutes presented a customised technique in both the DR and the ICR. One institute used the standard technique for the DR whilst in all 3 patients of the ICR deep tangential fields were used to irradiate both the breast and the IM nodes.

\begin{tabular}{|c|c|c|}
\hline General remarks & $\overline{\mathrm{DR}}$ & ICR \\
\hline Inirially no plans provided for arm 1 (withour LM-MS) & 3 & 0 \\
\hline Missing beam data / monitor unirs calcullations & 2 & 5 \\
\hline Flectron fields not planned & 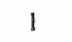 & 6 \\
\hline Separate electron and photon plans & 2 & 3 \\
\hline No drawing of internal structures & 0 & 14 \\
\hline Too few isodose lines & 11 & 6 \\
\hline Magnification factor different from plan to plan & 1 & 8 \\
\hline Dose prescription not according to ICRU recommendations & 2 & 3 \\
\hline Normalisation not correct & 8 & 6 \\
\hline Dose prescription not $25 \times 2 \mathrm{~Gy}$ & 4 & 2 \\
\hline No lung densicy correction & 2 & 1 \\
\hline Only inclusion of specific pacient sulggroups & 0 & 7 \\
\hline Excessive normal tissuc îradiarion & 3 & 5 \\
\hline Use of individual aratomical information & na: & 17 \\
\hline Specific remarks on the irradiation of the IM-MS lymph node chain & DR & ICR \\
\hline Length IM-MS, too long or too short & 9 & $3^{i+9}$ \\
\hline Position IM-MS field, cave underdose & 5 & 2 \\
\hline Overlap IM-MS and breast/thoracic wall fields, cave hot spot & 7 & 5 \\
\hline Exact match at junction, cave underdose & 5 & 6 \\
\hline IM-MS electron energy & 9 & $10^{\mathrm{a}}$ \\
\hline Dose prescription IM-MS photons & 8 & $9^{\infty}$ \\
\hline Proportion photons/electrous not $26 \mathrm{G} / 24 \mathrm{~Gy}$ & 4 & $8^{4}$ \\
\hline Bolus limiturg IMC-dose & 1 & $a$ \\
\hline Remarks on the irradiation of the breast/thoracic wall & $\overline{\mathrm{DR}}$ & ICR \\
\hline Dose to $[$ M-MS (unintended) $\geq 25 \%$ & 7 & 4 \\
\hline Supraclavicular irradiation & () & 4 \\
\hline Too low minimal dose ro breast/horacic wall CTV & 13 & 3 \\
\hline Wide fields around target volume & 3 & 1 \\
\hline Tangential fields at $180^{\circ}$ & 3 & 2 \\
\hline Too large or too low wedge fraction & 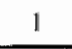 & 2 \\
\hline
\end{tabular}

${ }^{\circ}=$ all individualising based on anatomical information; $\mathrm{n}$ a. = aot applicable

Table 2: Remarks on the dumxyy run (DR) and on the individual case review (ICR). Numbers are numbers of institutes uere the specified remark was noted. 
Table 2 summarises a number of remarks and possible deviations from the protocol or from general guidelines. The most frequently noted deviation in the ICR was the lack of delineation of target volumes for the breast/thoracic wall and for the IM-MS lymph nodes in 14 institutes. All institutes which used a customised technique and 13 out of 15 using the standard technique localised the IM-MS nodes, mostly by CT-scanning, to individualise treatment set up and dose prescription. In the $\mathbb{L C R}$, there may have been a patient selection in 7 institutes, most often because all 6 patients were treated with a breast conserving approach. Most of the possible deviations for irradiation of the IM-MS target volume seen in the DR were also noted in the ICR. For breast/thoracic wall irradiation only, the dose was too low for 13 and 3 institutes in the DR and in the ICR respectively, generally due to too narrow margins. In 7/18 DR plans and in 4/15 ICR plans without IM-MS irradiation where we could estimate the dose to the IM-MS lymph node region, it exceeded $25 \%$.

\begin{tabular}{|c|c|c|c|c|c|c|}
\hline \multicolumn{7}{|c|}{ TANGENTIAL FIELDS TO BREAST/THORACIC WALL (13 institutes, 118 treatment plans) } \\
\hline Oof prescribed dose: & $<25 \%$ & $25-49 \%$ & $50-74 \%$ & $75-84 \%$ & $85-94 \%$ & $295 \%$ \\
\hline P(M) CAX & 24 & 4 & & & $1\left(\mathrm{c}^{-}\right)$ & 1 \\
\hline$P(3,3) C A X$ & 20 & 7 & 1 & $1\left(\mathrm{e}^{*}\right)$ & & 1 \\
\hline P(IM) CRAN & 26 & 2 & 1 & & & \\
\hline$P(3,3)$ CRAN & 22 & 5 & 1 & & & 1 \\
\hline Total & \multicolumn{2}{|c|}{$94 \%$} & \multicolumn{2}{|c|}{$3 \%$} & \multicolumn{2}{|c|}{$3 \%$} \\
\hline
\end{tabular}

ELECTRON FIELD TO THORACIC WALL (5 institutes, 18 treatment plans)

\begin{tabular}{|c|c|c|c|c|c|c|}
\hline \% Of prescribed dose: & $<25 \%$ & $25-49 \%$ & $50-74 \%$ & $75-84 \%$ & $85-94 \%$ & $\geq 95 \%$ \\
\hline PIN) CAX & & & 5 & & & \\
\hline$[P(3,3) C A X$ & & 1 & 2 & 2 & & \\
\hline$P(I N)$ CRAN & 2 & 2. & & & & \\
\hline $133 \mathrm{CRAN}$ & 2 & 2 & & & & \\
\hline Total & & & & & & \\
\hline
\end{tabular}

ALL. (18 institutes, 136 treatment plans)

\begin{tabular}{|c|c|c|c|c|c|c|}
\hline of prescribed dose: & $25 \%$ & $25-49 \%$ & $50-74 \%$ & $75-84 \%$ & $85-94 \%$ & $295 \%$ \\
\hline$P(\mathbb{M}) C A X$ & 24 & 4 & 5 & \multirow{4}{*}{3} & 1 & 1 \\
\hline$P(3,3) C A X$ & 20 & 8 & 3 & & & 1 \\
\hline $\mathrm{P}(\mathbb{M}) \mathrm{CWAN}$ & 28 & 4 & 1 & & & \\
\hline $\mathrm{P}(3,3) \mathrm{CKAN}$ & 24 & $?$ & 1 & & & 1 \\
\hline Torat & \multicolumn{2}{|c|}{$87 \%$} & \multicolumn{2}{|c|}{$10 \%$} & \multicolumn{2}{|c|}{$3 \%$} \\
\hline
\end{tabular}

Table 3 a: Number of treatment plans with the dose in $\%$ of the prescribed dose at the internal mammary lympls node bain for anm I (no IM-MS theatwent) in the dummy run. See Materials and Methods section and froure 1 for defivition of points $P(M M)$ and $P(3,3) ; C A X=$ centrals to the medial part of the mastectomy san. For 1 institute, doses conld not be calculated. 


\subsection{Dose to the IM-MS region in both randomisation arms:}

The doses at the points $P(I M)$ and $P(3,3)$ could be calculated for 18 to 19 institutes for the DR, arm 1 and 2 respectively and for 15 institutes for the ICR. The doses are displayed in Tables $3 a$ and $3 b$ for the treatment plans 'without IM-MS irradiation' and in Tables $4 a$ and $4 b$ for the treatment plans 'with IM-MS irradiation'. In Tables $3 a$ and $3 \mathrm{~b}$ results are shown for the plans with tangential beam techniques and the plans with electron field techniques separately. In the latter category a higher percentage of plans showed an unintended high dose to the points $P(I M)$ and $P(3,3)$. 'Tables $4 a$ and $4 b$ illustrate that the variability of the calculated dose is larger among institutes that used a standard technique as compared to those using customised techniques. In the latter less underdose was noted to the $P(I M)$ and $P(3,3)$ points.

\begin{tabular}{|c|c|c|c|c|c|c|}
\hline \multicolumn{7}{|c|}{ TANGENTIAL FIELDS TO BREAST/THORACIC WALL (13 institutes, 109 treatment plans) } \\
\hline 9. Of prescribed dose: & $<25 \%$ & $25-49 \%$ & $50-74 \%$ & $75-840 \%$ & $85-94 \%$ & $295 \%$ \\
\hline $\mathrm{P}(\mathrm{IM}) \mathrm{CAX}$ & 32 & 4 & 4 & & & 1 \\
\hline $\mathrm{P}(3,3) \mathrm{CAX}$ & 28 & 8 & 3 & & & 1 \\
\hline P(IM) CRAN & 13 & 1 & & & & \\
\hline$\underline{P(3,3) C R A N}$ & 13 & 1 & & & & \\
\hline Toral & \multicolumn{2}{|c|}{$92 \%$} & \multicolumn{2}{|c|}{$6 \%$} & \multicolumn{2}{|c|}{$2 \%$} \\
\hline
\end{tabular}

ELECTRON FIELD TO THORACIC WALL (2 institutes, 6 treatment plans)

\begin{tabular}{|c|c|c|c|c|c|c|}
\hline \% Of prescribed dose: & $<25 \%$ & $25-49 \%$ & $50-74 \%$ & $75-84 \%$ & $85-94 \%$ & $\geq 95 \%$ \\
\hline $\mathrm{P}(\mathrm{MM}) \mathrm{CAX}$ & & 1 & 1 & & & \\
\hline $\mathrm{P}(3,3) \mathrm{CAX}$ & & 1 & & & & 1 \\
\hline $\mathbb{P}(\mathbb{M})$ CRAN & & & $\mathbb{1}$ & & & \\
\hline $\mathrm{P}(3,3) \mathrm{CRAN}$ & & 1 & & & & \\
\hline Toral & \multicolumn{2}{|c|}{$50 \%$} & \multicolumn{2}{|c|}{$33 \%$} & \multicolumn{2}{|c|}{$17 \%$} \\
\hline
\end{tabular}

ALL (15 institutes, 115 treatment plans)

\begin{tabular}{|c|c|c|c|c|c|c|}
\hline$\%$ Of prescribed dose: & $<25 \%$ & $25-49 \%$ & $50.74 \%$ & $75-84 \%$ & $85-94 \%$ & $295 \%$ \\
\hline$P(I M) C A X$ & 32 & 5 & 5 & & & 1 \\
\hline $\mathrm{P}(3,3) \mathrm{CAX}$ & 28 & 9 & 3 & & & 2 \\
\hline P(IM) CRAN & 13 & 1 & 1 & & & \\
\hline $\mathrm{P}(3,3)$ CRAN & 13 & 2 & & & & \\
\hline Total & \multicolumn{2}{|c|}{$90 \%$} & \multicolumn{2}{|c|}{$7 \%$} & \multicolumn{2}{|c|}{$3 \%$} \\
\hline
\end{tabular}

Table 3b: Number of trament plans with the dose in \% of the prescribed dose at the internal mammary lymph node dain for arm I (no IM-MS treatment) in the individual case revicw. See Materials and Methods section and figure 1 for definition of points $P(I M)$ and $P(3,3) ; C A X=$ central slice; $C R A N=$ cranial slice. For 4 institutes, doses could not be calculated. 


\begin{tabular}{|c|c|c|c|c|c|c|}
\hline \multicolumn{7}{|c|}{ STANDARD IECHNIQUE (15 institutes, 98 treatmient plans) } \\
\hline \% OY prescerbed dorst: & $25 \% \%$ & $25-49 \%$ & $50-74 \%$ & $75-840 \%$ & $85-94 \%$ & $295 \%$ \\
\hline$P(I M) C A X$ & \multirow{4}{*}{\multicolumn{2}{|c|}{2}} & \multirow{3}{*}{5} & \multirow{4}{*}{4} & 2 & 23 \\
\hline$P(3,3) C A X$ & & & & & 4 & 12 \\
\hline P(M) CRAN & & & & & 2 & 22 \\
\hline$P(3,3)$ CRAN & & & 5 & & 5 & 10 \\
\hline Toul & \multicolumn{2}{|c|}{$2 \%$} & \multicolumn{2}{|c|}{$16 \%$} & \multicolumn{2}{|c|}{$82 \%$} \\
\hline \multicolumn{7}{|c|}{ CUSTOMISED TECHNIQUES (4 institutes, 32 treatment plans) } \\
\hline \% Of prescriberl dose: & $25 \%$ & $25-49 \%$ & $50-7.79 \%$ & $75-84 \%$ & $85-94 \%$ & $295 \%$ \\
\hline$P(I M) C A X$ & & & & & & 8 \\
\hline $\mathrm{P}(3,3) \mathrm{CAX}$ & & & 2 & 2 & 3 & 1 \\
\hline $\mathrm{P}(\mathrm{M}) \mathrm{CRAN}$ & & & & & 1 & 7 \\
\hline$P(3,3) \mathrm{CRAN}$ & & & 1 & 2 & 5 & \\
\hline Traal & & & & & & \\
\hline \multicolumn{7}{|c|}{ All. (19 institutes, 130 treatment plans) } \\
\hline \%of orescribed dosie: & $<25 \%$ & $25-49 \%$ & $50-74 \%$ & $75-84 \%$ & $85-94 \%$ & $295 \%$ \\
\hline$P(I M) C A X$ & & & & & 2 & 31 \\
\hline$P(3,3) \mathrm{CAX}$ & & & 7 & 6 & 7 & 13 \\
\hline$P[M] C R A N$ & & & & & 3 & 29 \\
\hline$P(3,3), C R A \mathbb{N}$ & & 2 & 6 & 4 & 10 & 10 \\
\hline Total & & & & & & \\
\hline
\end{tabular}

Table 4 a: Number of treatment plans with the dose in of the prescribed dose at the internal mammary lymph node clatin for am 2 (IM-MS treatment) in the dummy run. See Materials and Methods section and figure 1 for definition of points $P(I M)$ and $P(3,3) ; C A X=$ central slice; $C R A N=$ cranial slice. Doses could be calculated for all 19 institutes.

\begin{tabular}{|c|c|c|c|c|c|c|}
\hline \multicolumn{7}{|c|}{ STANDARD TECHNIQUE (11 institutes, 75 treatment pllans) } \\
\hline$\%$ Of prescribed dose: & $<25 \%$ & $25-49 \%$ & $50-74 \%$ & $75-84 \%$ & $85-94 \%$ & $\geq 95 \%$ \\
\hline$P(M M) C A X$ & & & & & 7 & 19 \\
\hline$P(3,3) \mathrm{CAX}$ & & & 3 & 4 & 5 & 11 \\
\hline P(IM) GRAN & & & 1 & & 3 & 9 \\
\hline$P(3,3) \mathrm{CRAN}$ & & & 2 & & 道 & 7 \\
\hline Tornl & \multicolumn{2}{|c|}{$0 \%$} & \multicolumn{2}{|c|}{$13 \%$} & \multicolumn{2}{|c|}{$87 \%$} \\
\hline
\end{tabular}

CUSTOMISED TECHNIQUES (4 institutes, 38 treatment plans)

\begin{tabular}{|c|c|c|c|c|c|c|}
\hline Yo Of prescribed dose: & $<25 \%$ & $25-49 \%$ & $50-74 \%$ & $75-84 \%$ & $85-94 \%$ & $\approx 95 \%$ \\
\hline$P(M) C A X$ & & & \multirow{4}{*}{\multicolumn{2}{|c|}{1}} & & 12 \\
\hline$P(3.3) C A X$ & & & & & 1 & 10 \\
\hline PIIM) CRAN & & & & & & 7 \\
\hline P(3.3) CANN & & & & & 2 & 5 \\
\hline Total & \multicolumn{2}{|c|}{$0 \%$} & \multicolumn{2}{|c|}{$3 \%$} & \multicolumn{2}{|c|}{$970 \%$} \\
\hline
\end{tabular}

\begin{tabular}{|c|c|c|c|c|c|c|}
\hline \multicolumn{7}{|c|}{ ALL (15 institutes, 113 treatment plans) } \\
\hline 36 Of prescribed dose: & $<25 \%$ & $2549 \%$ & $50-74 \%$ & $75-84 \%$ & $85-94 \%$ & $\geq 95 \%$ \\
\hline$P(I M) C A X$ & & & & & 7 & 31 \\
\hline$P(3,3) \mathrm{CAX}$ & & & 4 & 4 & 6 & $2 \mathbb{I}$ \\
\hline P(IM) CRAN & & & 1 & & 3 & 16 \\
\hline$P(3,3)$ CRAN & & & 2 & & 6 & 12 \\
\hline Tonal & \multicolumn{2}{|c|}{$0 \%$} & \multicolumn{2}{|c|}{$10 \%$} & \multicolumn{2}{|c|}{$90 \%$} \\
\hline
\end{tabular}




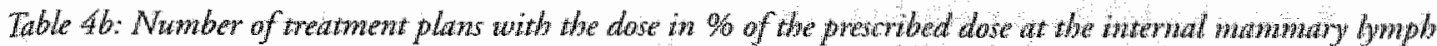

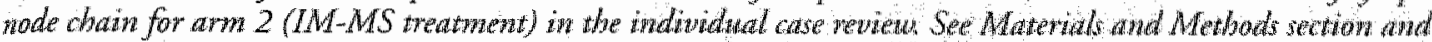

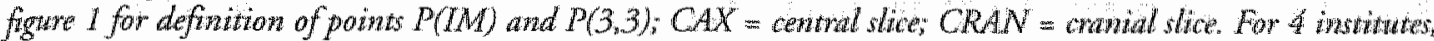
doses could not be calculated.

\subsection{Potential influence on trial outcome:}

For the above mentioned calculated dose variation to the IM-MS nodes in each randomisation arm we calculated the potential impact on the final outcome of the trial (Tables $5 \mathrm{a}$ and $5 \mathrm{~b}$ ). Taking the above-mentioned assumptions into account, the dilution effect of the dose variation onto the expected survival difference can be estimated. Assuming the same rates of deviations as assessed by the DR and the ICR respectively, the expected true 10-year survival benefit of $4 \%$ with IM-MS irradiation would be reduced to only $3.1 \%$ and $3.5 \%$ respectively due to "overdose" in the control arm and "underdose" in the treatment arm.

\begin{tabular}{|c|c|c|c|c|}
\hline \multicolumn{5}{|c|}{ ARM I: BREAST/THORACIC WALL ONLY } \\
\hline$\%$ Of prescribed dose: & $<50 \%$ & $50-84 \%$ & $\geq 85 \%$ & \\
\hline$\%$ of anses & 87 & 10 & 3 & \\
\hline 10 -Year survival & 75 & 75.4 & 79 & \\
\hline Toral & 65.3 & 7.5 & 24 & $=75.2 \%$ \\
\hline \multicolumn{5}{|c|}{ ARM II: BREAST/THORACIC WALL + IM-MS } \\
\hline \% Of prescribed dose: & $<50 \%$ & $50-84 \%$ & $285 \%$ & \\
\hline of cases & 2 & 17 & 81 & \\
\hline 10-Year survival & 75 & 75.4 & 79 & \\
\hline Totall & 1.5 & 12,8 & 64.0 & $=78.3 \%$ \\
\hline Difference & & & & $=3.1 \%$ \\
\hline
\end{tabular}

Table 5a: Theoretical influence of the actual dose at the IM-MS region as measured with the dummy run procedure on the estimated 10 -year survival, assuming that a dose $<50 \%$ to the IM-MS region has no effect, a dose of $50-84 \%$ bas a $10 \%$ effect and a dose $\geq 85 \%$ has a full effect, based on a 10 -year sumvival of $75 \%$ in the contral group and a $4 \%$ absolute improvement from 75 to $79 \%$. The real difference in 10 -year survival of $4 \%$, at the level of significance, will be observed as a difference in 10-year survival of $3.1 \%$.

\begin{tabular}{|c|c|c|c|c|}
\hline \multicolumn{5}{|c|}{ ARM I: BREAST/THORACIC WALL ONLY } \\
\hline$\%$ Of prescribed dose: & $50 \%$ & $50-84 \%$ & $285 \%$ & \\
\hline$\%$ of cases & 90 & 7 & 3 & \\
\hline 10- Year survival & 75 & 75.4 & 79 & \\
\hline Total & 67.5 & 5.3 & 2.4 & $=75: 2 \%$ \\
\hline \multicolumn{5}{|c|}{ ARM II: BREAST/THORACIC WALL + IM-MS } \\
\hline$\%$ Of prescribed dose: & $<50 \%$ & $50-840 \%$ & $2885 \%$ & \\
\hline$\%$ of cases & 0 & 10 & 90 & \\
\hline 10-Year surwival & 75 & 75.4 & 79 & \\
\hline Total & 0 & 7.6 & 71.1 & $=78.7 \%$ \\
\hline Difference & & & & $=3.5 \%$ \\
\hline
\end{tabular}

Table 56: Theoretical influence of the actual dose at the IM-MS region as measured with the individual case 
review procedure on the estimated 10-year survival, assuming that a dose $<50 \%$ to the IM-MS region bus no effect, a dose of $50-84 \%$ has a $10 \%$ effect and a dose $\geq 85 \%$ bas a full effect, based on a 10 -year survinal of $75 \%$ in the control grout and a $4 \%$ absolute improvement from 75 to $79 \%$. The real difference in 10-year survival of $4 \%$, at the level of significance, will be observed as a difference in 10-year survival of $3.5 \%$.

The trial is planned to detect a $4 \%$ difference with $80 \%$ power at the 0.022 significance level and 1000 events are needed for that purpose. To detect the smaller differences of $3.1 \%$ and $3.5 \%$ under the same conditions, a total of 1660 and 1420 events would be needed respectively. If the analysis was carried out as planned, whenever 1000 events are available, the trial would only have $55.8 \%(63.6 \%)$ power to detect the "diluted difference" of $3.1(3.5 \%)$ resulting from deviations from protocol dosing recommendations.

\section{Discussion}

In both the DR and the ICR, a significant number of mostly minor deviations from the protocol guidelines were found. Most of these deviations are due to ambiguities in the protocol, to not adhering to the ICRU dose prescription guidelines $[21,22]$, and for some institutes to a lack of familiarity with irradiating the complex set of target volumes formed by the lymph nodes and the breast/thoracic wall.

A rather typical problem when using the standard technique for IM-MS irradiation together with breast/chest wall irradiation is the matching of the field junctions. The technique used was the same for the DR and for the ICR for all but one institute, but only in the ICR we could evaluate the use of anatomical information. All institutes using a customised technique and 13 out of 15 using the standard technique individually localised the IM-MS nodes, mostly by CT-scanning. The treatment plans provided by the 4 institutes using a customised technique all showed an improved dose distribution at the junction of the different fields.

A majority of the deviations seen in the DR was also noted in the ICR. Many of those could be explained by individualising trearment plans based on anatomical information, which only became clear in the ICR. Several other deviations were noted in a different frequency in the DR and the ICR. The most remarkable finding was that in the ICR, the target volumes for the breast/thoracic wall and for the IM-MS lymph nodes were not drawn on the treatment plans in 14 institures. This might, even when using CT-based patient anatomy clearly visible on the TPS screen, hamper proper treatment prescription and evaluation. Therefore, when writing future protocols, more attention should be paid to the proper definition and delineation of target volumes. The simplest way for this 
might be to recommend a strict adherence to ICRU guidelines, although discrepancies will always occur because of the different interpretation of the clinical data by different clinicians. Some of the most common general deviations only hampered the evaluation of the DR and the ICR itself: too few isodose lines and a varying magnification factor of the plotted plan, depending on the TPS used by the institutes. Especially in the DR, the dose to the breast/thoracic wall was often too low. More often in the DR, the dose to the IM lymph node region was considered too high ( $225 \%$ ) for treatment plans without IM-MS irradiation. Furthermore, irradiation of the MS lymph node region for patients randomised to receive no IM-MS radiotherapy could only be seen in the ICR because this item was not checked in the DR.

Tables $3 a$ and $3 b$ illustrate that the use of electron field techniques to irradiate the thoracic wall more often leads to an unintended high dose to the points $P(I M)$ and $P(3,3)$. Tables $4 \mathrm{a}$ and $4 \mathrm{~b}$ show that the calculated dose to these points is less variable among the institutes that used customised techniques to irradiate the IM-MS lymph nodes as compared to those that used the standard technique, especially in the ICR. In general, the calculated doses at the points $P(I M)$ and $P(3,3)$ are more according to the prescribed dose for the ICR patients compared to the DR, but they still showed a significant variation from the ideal situation where a dose of $\leq 10 \%$ in Arm 1 and $\geq 95 \%$ in Arm 2 is called for. Since the sample size of the trial is based on significance at a $4 \%$ measured difference in survival probability, a true $4 \%$ difference might thus lead to a (falsely) non significant outcome of the trial with 3,1\% according to the DR and 3,5\% in the ICR. Of course, these hypothetical calculations have to be considered with caution. However, they illustrate the possible influence of a limited variation in treatment variables on the results of every clinical trial, also when it includes surgery and chemotherapy.

We have to recognise that not all institutes completed the DR and the ICR. However; since the participation to the DR was very high and the 20 participants to the ICR are the ones with the highest accrual (76\%), this can be judged as being of less importance. Moreover, as the DR and the ICR were performed in the early phase of the trial, recommendations for improvements could be given to all the parricipants before a significant number of patients were accrued. These recommendations were given on an individual basis after the review of the QA data and during the plenary sessions of the meetings of the EORTC Radiotherapy Group. A letter with recommendations was sent already in November 1997 to all participants. Based primarily on the findings of the DR, a revised version of the trial protocol was prepared and distributed as early as in May 1999 , less than three years after the start of the trial and before the accrual of one third 
of the patients. By doing so, the QA procedures will have had a synergetic effect. It also encouraged those institutes which had not yet participated to the QA program to do so and to those which had started patient accrual to improve the compliance to the trial protocol and to refine the radiotherapy techniques used.

Although the DR is limited due to the use of patient contours without complete clinical and anatomical information, it allows the evaluation of radiotherapy techniques applied and the compliance to most of the protocol and treatment guidelines before running the trial. Thereby, adjustments and recommendations can be made before the accrual of the first patient. The ICR is very suitable to evaluate not only patient and tumour related data for eligibility but also to verify the application of the recommendations given after the evaluation of the DR. In general, the outcome of the evaluation of the ICR showed a lower number of protocol deviations and an improved inter-institutional dose variation than encountered in the DR. This confirms our expectation that our suggestions and recommendations to improve the dose distribution led to an improved adherence to protocol guidelines. This will ultimately increase the reliability of the final trial results. We are convinced that quality assurance, like performed in this large multicentre trial, is of great importance to all disciplines involved in the treatment of cancer patients.

\section{Acknowledgements}

The authors want to thank all the trial participants as well as Marianne Pierart, data manager at the EORTC Data Center. More members of the QA committee of whom the valuable input in the early phase of the QA program is very much appreciated are Vernon Vlaun, Karin De Winter and Marleen Van der Hulst, Tilburg; Jan Lagendijk, Utrecht; Coen Hurkmans, Eindhoven; Ben Mijnheer, AvL Amsterdam; Dominique Huyskens, Leuven. Vassilis Kouloulias worked in 2001 as Emmanuel van der Schueren fellow in

Quality Assurance at the EORTC Data Center, supported by a grant from the "Vlaamse Kankeriga".

\section{References}

1. 1. Cuzick I. Stewart H, Rutquist LE, et al. Cause-specific mortality in long-term survivors of breast cancer who participated in trials of tadiotherapy. J Clin Oncol 1994, 12,447-453.

2. Overgard M, Hansen PS, Overgaard J。 et al. Postoperative radiorherapy in high-risk premenopausal women with breast cancer who receive adjuvant chemotherapy. $N$ Engl J Med 1997, 337, 949-955.

3. Ragaz I, Jackson SM. Le N, et al. Adjuwant radiotherapy and chemorherapy in node-positive premenopausal women with breast cancen $N$ Engl J Med 1997, 337, 956-962.

4. Overgaard M, Jensen M-J, Overgaard J, et al. Postoperative radiotherapy in high-risk postmenopausal breastcancer parients given adjuvant Tamoxifen: Danish Breast Cancer Cooperative Group DBCG $82 \mathrm{c}$ randomised trial. 
Lancet $1999,353,1641-1648$.

5. Hfyris $I_{2}$ Overgaard $M$, Christensen J], et al. Morbidity and morcality of ischemic hearr disease in hightrisk breastcancer patients after adjuvant post-nnastectomy systemic treatment with of without radiotherapy: analysis: of DBCG $82 \mathrm{~B}$ and $82 \mathrm{C}$ randomised rials. Lancet $1999,354,1425-1430$.

6. Fowble $\mathbb{B}_{0}$ Hanlon $A$, Freedman $G_{\text {s }}$ et al. Internal mammary node irradiation neicher decreases distant metastases nor improves survival in stage I and II breast cancer. Int J Radiat Oncol Biol Phys 2000, 47, 883-894.

7. Early Breast Cancer Trialists ${ }^{2}$ Collaborative Group. Favourable and unfavourable effects on long-term strvival of radiotherapy for early breast cancer: an overview of the randomised trials. Lancet 2000, 355, 175741770.

8. Whelan TJ, Julian J, Wright J, et al. Does locoregional radiation therapy improve survival in breasr eancer? A meraanalysis. J Clin Oncol 2000, 18, 1220-1229.

9. Kanja $\mathrm{H}$, Mauna $\mathrm{P}$. Tangential breast irradiation with or without internal nammary chaim irtadiationy results of a randomized trial. Radiorher Oncol 1995, 36, 172-176.

10. Stenmer SM, Rizel S, Hardan I, er al. The vole of irradiation of the intemal mammary lymph nodes in high-risk stage III to IILA breast cancer parients after high-dose chemotherapy: a prospective sequential nonmandomized study. I Clin Oncol 2003, 21, 2713-2718.

11. Van den Bogaert W, Struikmans H, Fourquet A, Bartelink H. Internal mammary and medial supraclavicular (IMMS) lymph node chain irradiation in stage I-III breast cancer. A phase III randomised trial of the EORTC, protocol 22922/10925. May 1996, revision May 1999.

12. Lievens $Y$, Poortmans $P$, Van den Bogaert W. A glance on quality assurance in $\mathbb{E O R T C}$ study 22922 evaluating techniques for internal mammary and medial supraclavicular lymplu node chain irradiation in breast cancer. Radiother Oncol 2001, 60, 257-265.

13. Poortmans PMP, Venselaar JLM, Struikmans $H$, et al. The potential impact of treatment variations on the tesults of radiotherapy of the internal mammary lymph node chain: A quality-assurance report on the dummy tun of EORTC phase III randomized trial 22922/10925 in srage I-fLII breast cancer. Int J Radiat Oncol Biol Phys 2001, 49, 1399-1408:

14. Bolla $M$, Bartelink $H$, Garavaglia $G$, et al. EORTC guidelines for writing protocols for clinical trials of radiotherapy. Radiother Oncol 1995, 36, 1-8.

15. Hurkmans CW, Borger JH, Bos LJ, et al. Cardiac and lung complication probabilities after breast cancer irradiation. Radiother Oncol 2000, 55, 145-151.

16. Kouloulias VE, Poormars PM, Bernier J, er al. The quality assurance programme of the Radiotherapy Group of the European Organisation for Research and Treatment of Cancer (EORTC): a critical appraisal of 20 years of continuous efforts. Eur J Cancer 2003, 39, 430-437.

17. Gomola I, Van Dam J, Isern-Verdum J, et all. Externad audits of electron beams using mailed TLD dosimetry: preliminary results. Radiocher Oncol 2001, 58, $163-168$.

18. Poortmans $P_{x}$ Kouloulias VE, Venselaar JLs er al. Quality assurance of EORTC trial 22922/10925 investigaring the role of internal mammary - medial supraclavicular inadiation in stage $\mathbb{I} I l l l$ breast cancer: the individual case review. Eur J Cancer 2003, 39, 2035-2042.

19. Pierce LJ, Richer AS. Postmastectomy radiotherapy: more than locoregional control. I Clin Oncol 1994, 12, 444446.

20. Struikmans $H$, van Rijk PP. Oprimizing radiotherapy of the internal mammary chain in breast carcinoma by scintigraphy, Radiother Onool 1996, 41, 15-20.

21. ICRU Report 50. Prescribing, Recording and Reporting Photon Beam Therapy. International Commission on Radiation Units and Measurements. 7910 Woodmont Avenue, Berhesda, Maryland 20814, USA.

22. ICRU Report 62. Prescribing, Recording and Reporting Phoron Beam Therapy (Supplement to ICRU Report 50). International Commission on Radiarion Units and Measurements. 7910 Woodmont Avenue, Bethesda, Maryland 20814, USA. 


\section{CHAPTER 10}

The Quality Assurance Programme of the Radiotherapy Group of the European Organisation for Research and Treatment of Cancer: Past, Present and Future.

P. M. Poortmans, J. B. Davis, F. Ataman, J. Bernier, J.-C. Horiot, for the EORTC Radiotherapy Group.

Eur J Surg Oncol: accepted, 2004. 


\section{Abstract}

As early as in 1982, the European Organisation for Research and Trearment of Cancer Radiotherapy Group established a quality assurance programme. In the course of 20 years, quality assurance procedures have become a vast and important part of the activities of the group. Today, the membership committee uses standard procedures based on minimal requirements to evaluate current members and new membership applications. Moreover, for every new trial, specific quality assurance procedures are an integral part of the preparation of the protocol and executed under the responsibility of the study co-ordinator. With the growing complexity of the radiotherapy techniques used in the framework of the more recent trials, quality assurance procedures have also become more complex including trial specific phantom based measurements. Future ways to evaluate all steps of the radiotherapy process using a common platform connecting all users with the Internet are currently under development.

Keywords: Quality assurance; radiotherapy; European Organisation for Research and Treatment of Cancer (EORTC); clinical trials; review. 


\section{Introduction}

All patients, including those with cancer, need to be treated according to the highest standards, and preferably in the framework of a well designed clinical trial which gives us the opportunity to learn more from the disease and optimise its treatment. For the reliability of the interpretation of the results of a clinical trial, the design of the protocol, the way that it is conducted and the evaluation of the data are of utmost importance. Quality assurance (QA) procedures are able to identify flaws in the design of a trial as well as to assess if deviations from the guidelines of the protocol are caused by misinterpretation of the trial prescriptions. Only the results of a well conducted trial where patients are treated in standardised and technically optimal conditions will be of use for the interpretation of the results and find general acceptance.

It is well documented that patients who participate in a clinical trial often have a better outcome than orher similar patients do. This variation not only concerns the effectiveness of the therapeutic management in terms of cure rate, but also the risk for severe complications and the outcome in terms of quality of life. The better outcome of trial parients can be explained by a patient selection bias but also by the use of more precise standards, superior QA of the diagnostic and therapeutic procedures and more attention from health care workers in centres which participate in clinical trials. [1]

In this perspective, the European Organisation for Research and Treatment of Cancer Radiotherapy Group (EORTC-RTG) has developed, over the past 20 years, a comprehensive QA programme to validate the reliability of their clinical trials. Part of the QA procedures were and are related to the structure and the functioning of the department including the radiation physics parameters and another part is specific to the cinical trials. Reviews of the QA programme of the EORTC-RTG have been reported elsewhere. $[2,3,4,5,6]$.

\section{The history of quality assurance in the EORTC Radiotherapy Group}

\subsection{Site visits (1982-1987)}

In 1982 the EORTC-RTG activated its QA programme. Initially, various member centres were visited by a team of radiation oncologists and radiation physicists. The evaluation included three steps: an evaluation of the infrastructure, staffing levels, mechanical and dosimetric integrity of treatment units, and a check of the data in the clinical and radiotherapy charts. The study included 17 centres that were visited by a group of experts in radiotherapy and radiation physics from January 1982 to December 
1984. Large variations were observed in the workload as expressed in the number of patients treated per year per radiation oncologist, radiation physicist and radiation technologist. Also for the infrastructure a wide variation in the number of treatment machines existed with major problems for $5 / 17$ centres making it difficult to comply with the requirements of the EORTC-RTG. [7] The dosimetric evaluation showed. deviations for a number of scanning electron beams, and for the flatness and the symmetry of as well the X-ray as the electron beams. [8] No major deviations related to absorbed dose calibration or calculation in an anatomical phantom, containing a tonsillar tumour and a homolateral subdigastric node were found. This investigation led to the development of the concept of what was later named the "dummy run" procedure: investigators were asked to treat a dummy patient (the Alderson anatomical phantom) as if it was a true patient entered in an active protocol (in that case EORTC protocol No. 22791. comparing conventional versus twice a day fractionation in oropharyngeal cancers). Although the dose delivered was correct on the central axis in all cases, large differences in treatment planning were observed resulting in about one third of the cases in insufficient or excessive target volumes, exposing the "patient" to either increased failure or complication rates. [9] The evaluation of mechanical checks of megavoltage units and simulators showed that, in general, the deviations observed for accelerators and simulators were smaller than for cobalt units, possibly related to the advanced age (up to 20 years) of some of the latter units. [10]

\subsection{Thermoluminescent dosimetry (1987-today)}

In 1987, a mailed thermoluminescent dosimetry (TLD) programme for machine output check was started. This revealed a few large deviations $(>7 \%)$ between the dose measured and the dose stated by the centres. Based on this corrections were made by the participating centres. Subsequent mailings resulted in a decrease of the measured deviations and the standard deviation on subsequent mailings. Correlating information from the TID measurements with the on-site visits led to further decrease in the beam output deviations. [11] In 1993, a report on the mailed TLD results of radiotherapy centres in Europe showed that the large majority of the beams (23/25) with deviations > $3 \%$ were from centres which had not participated in external audits in the 5 years preceding the audit. [12] Based on the outcome of these QA procedures, a document with minimum requirements for $\mathrm{QA}$ in radiotherapy departments was published. [13] The mailed TLD dosimetry programme of the EORTC-RTG was later taken over by the European Society for Therapeutic Radiology and Oncology (ESTRO). [14,15] In 1996, a new evaluation of the profile of the radiotherapy departments participating in clinical trials of the EORTC-RTG was published. Compared with their earlier report, the large 
wariations in equipment number and age and in staffing levels among participating centres continued to exist; the number of cancer patients treated per year per radiation oncologist diminished slightly, especially in those centres which had experienced a considerable staff shortage; the most significant improvement was observed for the number of cases treated per year per member of the radiation physics team; the radiation technologist's workload showed the opposite trend and the situation for equipment remained unchanged. [16] Based on a consensus meeting in 1987, focusing on QA procedures in radiotherapy inside the EORTC, a report with recommendations and guidelines was published. [17] It was recommended that a centralised QA programme should include sire visits, evaluations of the dosimetry and a review of the radiotherapy treatment records. Criteria for ranges of variation for dose prescription and delivery, patient immobilisation as well as the verification by portal films were included. Emphasis was given to the mandatory use of the International Commission on Radiation Units and Measurements (ICRU) recommendations. [18,19]

\subsection{Trial oriented procedures (1987-today)}

In 1987 the Radiotherapy Group initiated trial oriented QA procedures, initially consisting of the evaluation of a fictitious parient (dummy run) to identify, prior to protocol activation, any deviation which might occur. As protocol non-compliance may be due to the guidelines being not clearly written or to a different incerpretation of the guidelines from that intended by the study co-ordinator, there would be ample time to take corrective action. Similarly, any deviation in the dosimetry or treatment techniques can be corrected and advice given if deemed necessary. Shortly after, in 1989, patientoriented QA reviews were activated to search for random errors. These so-called individual case teviews (ICR) included a review of patient, tumour and treatment related parameters to evaluate and to improve the compliance of the participating centres to study protocols. A consensus meeting including the participation of surgeons, pathologists, radiation oncologists, physicists and radiation technologists was held to develop treatment and QA guidelines to be applied in the framework of clinical trials in early breast cancer. [20] During a subsequent QA meeting of the Radiotherapy Group in January 1993, three goals were defined: definition of minimum requirements for participating centres, evaluation of dose-volume effect in normal tissues and predictive tests for a better characterisation of individual radio-sensitivity. [21] Special attention was. also given to the definitions of acceptable variations, major and minor deviations of treatment parameters. The concept of a Master Protocol for phase III studies was realised; in order to make the work of future study co-ordinators easier and to improve the homogeneity of the protocol prescriptions of a clinical trial. [22] One of the first trials 
for which an extensive QA programme was organised is EORTC trial No. 22881/10882 evaluating the value of a boost dose in breast conserving therapy for early breast cancer. $[23,24]$ For the dummy run, three transverse sections of a patient were sent to 16 participating centres with a request to make a 3-plane treatment plan, according to the protocol guidelines. The evaluation revealed a large variation in treatment techniques used, especially concerning the use of wedge filters. Further steps of the QA programme of this trial consisted of an ICR procedure and in vivo as well as phantom-based dosimetry studies. Three types of systematic protocol deviations were detected: deviations due to ambiguities in the protocol prescriptions; deviations not known to the centre, such as misinterpretation of trearment planning guidelines and the inability of an centre to cope with all protocol prescriptions for technical or logistic reasons. The first 2 types of deviations can be corrected by individual discussions, specific recommendations for the dose in the ICRU point and in vivo dosimetry. With respect to the third type it is up to the trial co-ordinator to accept or refuse a centre's participation, depending upon the relative importance of the particular deviation(s) for the trial end points. Later, the results of several other trial-bound QA procedures were reported and are under preparation: several aspects of QA in 4 consecutive dinical trials in prostate cancer (EORTC protocols No. 22863, 22911, 22961 and 22991) including dummy runs, individual case reviews and evaluation of technology questionnaires; an individual case review in EORTC protocol No. 22921 on preoperative radiotherapy with or without chemotherapy for resectable rectal cancer; a dummy run in EORTC protocol No. 22931, comparing postoperative radiotherapy alone to concomitant radio-chemotherapy for patients with locally advanced head and neck carcinomas; a dummy run and an individual case review in EORTC trial No. 22922/10925 investigating the role of adjuvant internal mammary and medial supraclavicular (IM-MS) irradiation in stage I-III breast cancer; a review of the medical files from EORTC trial No. 10853 investigating the role of radiotherapy in breast-conserving treatment for ductal carcinoma in situ of the breast. Similar findings were reported in most of these QA studies, including sometimes wide variations in the different diagnostic and treatment procedures raising the question of homogeneity and reproducibility. From the radiotherapy aspects, the most important and largest variations were observed in the delineation of target volumes. $[25,26,27,28,29,30,31,32]$ A lack of adherence to $\mathbb{C}$ CRU recommendations for dose prescription and reporting was noted as well. This emphasises the need for establishing clear and uniform guidelines for all steps of the diagnostic and the therapeutic procedures. For EORTC trial No. 22922/10925, theoretical calculations of the influence of the sub-optimal dose distribution in the IMMS region as calculated in the dummy run, revealed that a real survival benefit of $5 \%$ would be measured as only a $3.8 \%$ benefir, resulting in a false negative outcome of the 
primary trial endpoint. [29]

\section{The current standard quality assurance procedures}

The 1995-1996 period was characterised by the further development of QA procedures that had been validated during the three phases mentioned above with a particular emphasis on the mailed TLD programme and on trial-bound programmes including dummy-runs and individual case reviews. Since 2001, trial-bound QA procedures are greatly helped by the support of the Emmanuel van der Schueren fellowship in QA. In 2001, the results of the first large-scale mailed TLD evaluation of electron beams in reference centres and centres participating in EORTC trial No. 22922/10925 were reported: the standard deviation of the measured dose to the stated dose was $3.2 \%$, while in 7 of the 330 beams a deviation exceeding 10\% was observed. [33] Bentzen used radiobiological modelling of data from the TLD dosimetry study of the EORTC to estimate the possible clinical impact of QA programmes on local tumour control and on complication probability. For the $10 \%$ of the beams with the most pronounced underdose, the loss in tumour control probability could be up to $7-8 \%$. Similarly, in the $10 \%$ of the beams with the most pronounced overdose, the calculated increase in the probability of mild/moderate morbidity could be up to 19-22\%. Moreover, for severe morbidity the same beams raised the estimated probability of incidence of severe complications from $5 \%$ to $9-10 \%$. The loss of uncomplicated cure probability was estimated at $1 \%$ both for high and low energy beams. [34]

In 1994 ESTRO published an Advisory Report to the European Commission within the framework of the "Europe against Cancer" Programme [35]. The main item of this report was the documentation of guidelines for QA in the radiotherapy. This resulted in the establishment of a TLD laboratory for dose monitoring in radiotherapy centres throughout Europe. Today, ESTRO offers through EQUAL, an acronym for ESTRO Quality Assurance in radiotherapy, highly specialised skills and tools for various aspects of QA. [15]

The EORTC-RTG formed a Membership Commitree that evaluates all new applications for membership. Criteria for membership to the RTG are based on infrastructure and staffing levels. The committee can also be involved in discussions concerning participation in specific clinical trials involving specific radiotherapy techniques. Candidate members are asked to complete a questionnaire on infrastructure, staff and institutional QA procedures. Moreover, annual beam calibration done by ESTROEQUAL or an equivalent laboratory is requested. Based on the outcome of this 
evaluation, and if necessary after further discussion, a candidate member can be accepted. Recently, a technologically more advanced dummy run (DR) for the intensicy modulated radiotherapy (IMRT) treatment arm of the EORTC trial No. 22991 has been started. This trial investigates the value of short-term adjuvant hormonal therapy in early stage prostate cancer treated with 3-D conformal radiotherapy (3-DCRT) or IMRT. Because of the technical complexity, this is done in co-operation with the ESTRO QA office (EQART). This evaluation needs to include the inverse treatment planning algorithm and the treatment delivery by comparing the planned versus the measured dose distribution as well as the on-line treatment portal imaging verification. The OPERA phantom (Operational Phantom for European Radiotherapy Audits) is a phantom designed by EQART to check the dosimetric aspects of complex treatments. It has been adapted for IMRT and has been used in 5 key centres in Europe to establish the principle and functionality. The OPERA phantom will be sent to each participating centre to evaluate the accuracy of the IMRT inverse planning and the IMRT treatment delivery with the use of film dosimetry and TLD. The estimated cost of such complex dosimetry is bound to be high. It is expected that participating centres may have to bear some of the costs involved.

\section{Quality assurance in radiotherapy in the 21 st century}

Standards of care are constantly improving. In radiation therapy as for other treatment modalities, this has meant more complex treatments. These treatments are technically more demanding and are often more protracted to reduce unwanted side effects. Consequently, it is expected that future clinical trials will become more complicated. This trend can already be observed in the growing number of trials in which special techniques such as stereotactic radiotherapy and IMRT are used. Inevitably, this will be accompanied by a higher level of complexity of the QA procedures, which translates into a heavier burden and costs for the EORTC Data Centre and the participating centres alike. For the past few years, the RTG QA co-ordinators have been looking at ways of reducing the QA burden and costs without sacrificing accuracy and effectiveness.

\subsection{Data base on infrastructure}

One of the first steps will be the establishment of a dara bank of the infrastructure of the member centres. The infrastructure questionnaire has been adlapted and has recently been placed on the RTG website. This questionnaire is designed to acquire data on equipment, staffing levels, treatment techniques and QA procedures in member centres. The data bank will be regularly updated and will be available to all members. One advantage of 
such a data bank is that information deemed necessary for study and QA co-ordinators will be readily accessible without having to resort to asking each participating centre to fill in another questionnaire.

\subsection{Web-based quality assurance}

With the tremendous progress made in information technology, the propagation of computers and the ease of access to the Internet, it is clear that this points the way to future QA procedures. The EORTC has a long term collaboration with the National Cancer Institute (NCI), so it seemed logical to collaborate with them in QA matters as they have already developed a system for digital planning data transfer via the internet for many Radiation Therapy Oncology Group (RTOG) trials.

The Advance Technology Consortium (ATC) is funded by the NCI and consists of several existing partners, namely the Image-Guided Therapy QA Center (ITC), the Quality Assurance Review Center (QARC), the RTOG Radiological Physics Center (RPC) and the Resource Center for Emerging Technologies (RCET). The ATC is committed to using advanced medical informatics and aims at facilitating education, collaboration and peer review, providing an environment in which clinical investigators can receive, share and analyse treatment planning digital data, which can support clinical trial QA. The ATC also performs image and radiation therapy digital data management and is actively engaged in research and development in these areas. Its ultimate goal is to improve standards of care in management of cancer by improving the quality of clinical trial medicine. To achieve this, ATC focuses on electronic data exchange of treatment planning digital data between ATC QA centres and centres participating in clinical trials. Digital Image and Communication (DICOM) is used in all parts of the radiotherapy treatment planning including $\mathrm{CT}$ images, anatomical structure sets, radiotherapy plans, images (DRR, BEV etc) and doses. Data of patients entered into clinical trials can be uploaded via the Internet to a central server pending review by a QA group. This server could be in any centre, at the EORTC Data Centre, or at the ATC and is accessible to the study co-ordinators and QA group.

Centres will be credentialed prior to being allowed to submit data. This will be done after completion of a facility questionnaire and after successfully completing and submitring a protocol specific DR. It may be possible to use the data bank described above instead of the facility questionnaire to access this information. Treatment planning data will be submitted in digital format, either in the RTOG specification for network format or in DICOM RT format (in compliance with the ATC's DICOM RT conformance statement). The digital data requirements include protocol compliant images (CT, MRI 
etc), protocol compliant contours using standard names for body outline, target volumes (GTV, CTV, PTV) and all specified organs at risk (OAR), beam geometry and doses for all courses, DRR or digital portal images for each beam and a dose-volume histogram (DVH) for PTV and OAR. The trial-bound QA is centre oriented (dose at reference point) as well as trial specific (DR and ICR). Data will be sent from the QA team to the participating centre and vice versa. Some data, like some clinical data and case report forms, may be submitted electronically or as a hardcopy.

\section{Discussion}

In the past, some problems concerning protocol inconsistencies and routine clinical practice have been noted. Potential systematic protocol deviations possibly leading to false negative/positive results were often detected. Special attention must be given to the variations in the delineation of target volumes and to the adherence to ICRU recommendations for dose prescription and reporting. The possible influence of deviations in treatment delivery on the end-results of studies has been estimated and this fact has emphasised the immense value of QA in clinical trials.

As a rule, a QA programme is implemented for every clinical trial activated by the EORTC-RTG and has resulted in a specific field of clinical research to develop methodologies that are now gradually transferred from clinical research entities to the entire radiotherapy community. To be effective, the QA programme must be implemented prior to protocol activation or as early as possible in the course of a clinical trial. It should also include a direct interactive feedback procedure. The main aspect of $\mathrm{QA}$ is not only to check protocol compliance, or detect an inconsistency but also to give advice, aiming to improve protocol compliance and ensuring that corrections are made as soon as possible. Thus a QA structure should be friendly, informal and confidential, with the ultimate aim of improving the quality of clinical practice. Therefore, published data from a QA procedure should always be in an anonymised form. The QA programme in the EORTC-RTG is generally well accepted by all participants even though it adds to the burden of busy clinics. It is considered by everyone involved to be mandatory for the validity of the clinical data collected from different departments with different resources and working in different ways.

The QA programme of the EORTC-RTG can also be a valuable tool in harmonising the infrastructure, human resources and the execution of radiotherapy both in member and non-member departments. This can lead to the improvement, on a very broad scale, of treatment outcome in terms of local control, survival, complications and quality of life. 
Mailed TLD verification of the beam output of radiotherapy machines should be an integrall part of basic QA procedures in radiotherapy.

Today, the main axes of research in QA could be articulated around the following issues:

- Cost-benefit analysis of the evaluation of case report forms, DR and ICR procedures.

- An update of information on required radiotherapy infrastructure in EORTC centres based on mailed questionnaires possibly in collaboration with ESTRO.

- The estimation of the influence of treatment variations and inter-institutional variability on the outcome of clinical trials in terms of tumour control and normal tissue complication probability.

- The co-operation in the field of QA with other oncology treatment specialities like surgery, medical oncology and pathology.

In conjunction with the NCI/EORTC collaborative project, the use of Telematics will be investigated for teleconferencing QA audits and for DICOM based information exchange between the QA team and the participating departments, especially when new radiotherapy techniques are involved.

Quality assurance has a cost. Some of it is provided by the participating institutions with the competence and time spent by investigators (radiation oncologists and radiation physicists). Another part is sometimes funded by the pharmaceutical industry when relevant to the protocol (e.g. for some of the prostate cancer trials). Funding can also come from charities (e.g. cancer leagues) for a specific programme submitted to a scientific committee or for a specific fellowship: The Flemish Cancer League is funding the "Emmanuel van der Schueren fellowship for quality assurance" which plays a major" role in co-ordinating quality assurance programmes in the EORTC-RTG. However, these sources of funding are not permanent. The QA budget must be sought for every year and this may jeopardise our efforts in implementing our know how in each protocol.

\section{Conclusions}

Quality Assurance in clinical trials can be a useful tool for increasing the quality of radiotherapy in general. Study co-ordinators, QA team and participating centres must communicate on an individual basis. Free exchange of ideas between members in general and the publication of findings provide a platform for sharing knowledge and experience. The level of appropriate quality assurance in clinical trials is highly dependent on the complexity of the trial. It should be comprehensive and it effects should also extend to 
the treatment of all patients. By doing this, efficient quality assurance in clinical trials will improve the overall quality of treatment for most patients at an acceptable cost.

\section{Acknowledgements}

The authors wish to thank the numerous colleagues who were actively or passively involved in the organisation and execution of quality assurance in the EORTC Radiotherapy Group, including but not limited to: Harry Bartelink, Guido Garavaglia, Jean-Yves Giraud, Frances Godson, Karl-Axel Johansson, Vassilis Kouloulias, Philippe Maingon, Marianne Pierart, Jan van Dam, Geertjan van Tienhoven and the late Emmanuel Van der Schueren. We also thank very warmly the Vlaamse Kankerliga for the ongoing financial support of the Emmanuel Van der Schueren fellowship (Dr. Farma Ataman) for Quality Assurance in radiotherapy and our colleagues from the ESTROEQUAL network and from the NCI-ATC consortium for the positive and fruitful collaboration. None of this would have been possible without the collaboration of the participating centres, to which we express a special note of gratitude.

\section{References}

1. Antman K, Amato D, Wood Wet al. Selection bias in clinical trials. J Clin Oncol 1985:3:1142-1147.

2. Horiot JC, wan der Schueren $\mathrm{E}$, Johansson $\mathrm{KA}$, Bernier J, Bartelink H. The programme of quality assurance of the EORTC radiotherapy group. A hiscorical review. Radiother Oncol 1993;29:81-84.

3. Meunier F, Horior JC. EORTC scientific strategy meering 25-26 March 1999. Eur \ Cancer 1999;35:1423-1430.

4. Bernier J, Horior JC, Poortmans .PM - Quality Assurance in radiorherapy: from radiation physics to patient- and trial-oriented control procedures. Eur J Cancer 2002;38:155-158.

5. Ottevanger PB, Therasse $\mathrm{P}$, van de Velde $\mathrm{C}$, Bernier J, van Krieken $\mathrm{H}$, Grol R, De Mulder $\mathrm{P}$. Quality assurance in clinical trials. Crit Rev Oncol Hematol 2003,47:213-235.

6. Kouloulias VE, Poortmans PM, Bernier I et al. The quality assurance programme of the Radiotherapy Group of the European Organization for Reseatch and Treatment of Cancer (EORTC): a critical appraisal of 20 years of continuous efforts. Eur I Cancer 2003;39:430-437.

7. Horiot JC, Johansson KA, Gonzalca DG, wan der Schueren E, van den Bogaert W, Noter G. Quality assurance control in the EORTC cooperative group of radiotherapy. 1. Assessment of radiotherapy staff and equipment. European Organization for Rescarch and Treatment of Cancer. Radiother Oncol 1986:6275-284.

8. Johansson KA, Horio JC, Van Dam J, Lepinoy D. Sentenac I, Sernbo G. Quality assurance control in the EORTC cooperarive group of radiotherapy. 2. Dosimerric intercomparison. Radiother Oncol 1986;7:269-279.

9. Johansson KA, Horior JC, van der Schueren E. Quality assurance control in the EORTC cooperative group of radiotherapy. 3. Intercomparison in an suatomical phancom. Radiother Oncol 1987;9:289-298.

10. Van Dam J. Johansson KA, Bridier A, Sernbo G, Hansson U. EORTC radiotherapy group quality assurance: mechanical checks and beam alignments of megawoltage equipment. Radiother Oncol 1993;29:91-96.

11. Hansson U, Johansson KA, Horiot JC, Bernier J. Mailed TL dosimerry programme for machine outpur check and clinical in the application in the EORTC radiotherapy group. Radiother Oncol 1993;29:85-90.

12. Dutreix A, van der Schueren $E$, Derreumaux $\$$, Chavaudra J. Preliminary results of a quality assurance network for radiotherapy centres in Europe. Radioner Oncol 1993;29:97 101.

13. Horiot JC, Bernier J, Johamsson KA, van der Schueren E, Bartelink I-I. Mininum requirements for quality 
assurance in radiotherapy: Radiother Oncol 1993;29:103-104.

14. Derreumaux $S$, Chavaudra J, Bridier A, Rossetri V. Dutrex A. Furopean quality assurance network for radiotherapy: dose measurement procedure. Phys Med Biol 1995;40:1191-1208.

15. Ferreira IH, Durreix A, Bridier A, Chavaudra J. Svensson H. The ESTRO QUALiry assurance nerwork (EQUAL). Radiocher Oncol 2000;55:273-284.

16. Bernier J, Horior JC, Bartelink H er al. Profile of radiotherapy departments contributing to the Cooperarive Group of Radiotherapy of the European Organization for Research and Treatment of Cancer. Int I Radiat Oncol Biol Phys $1996: 34: 953-960$.

17. Johansson KA, Hanson WF, Horior JC. Workshop of the EORTC Radiotherapy Group on quallity assurance in cooperative trials of radiotherapy: a recommendation for EORTC Cooperative Groups. Radiother Oncol $1988 ; 11: 201-203$.

18. ICRU Report 50. Prescribing, Recording and Reporting Photon Beam Therapy. Intemational Commission on Radiation Units and Measurements. 7910 Woodmont Avenue, Bethesda, Maryland 20814, USA.

19. ICRU Report 62. Prescribing, Recording and Reporting Photon Beam Therapy (Supplement to ICRU Report 50). International Commission on Radiation Units and Measurements. 7910 Woodmont Avenue, Bethesda, Maryland 20814, USA.

20. Bartelink H, Garavaglia $\mathrm{G}$, Johansson KA et al, Qualicy assurance in conservative treatment of early breast cancer. Report on a consensus meeting of the EORTC Radiotherapy and Breast Cancer Cooperative Groups and the EUSOMA (European Sociery of Mastology). Radiother Oncol 1991;223323-326.

21. H. Bartelink, J. Bernier ${ }_{\mathrm{y}}$ J. Kurtz, G. Garavaglia, Radiother Oncol 1993;29:xi-xii.

22. Bolla $M$, Bartelink $H$, Garavaglia $G$ et al. EORTC guidelines for writing protocols for dinical trials of radiotherapy. Radiother Oncol 1995;36:1-8.

23. van Tienhoven $G$, van Bree NA, Mijnheer B], Bartelink H Quality assurance of the EORTC trial 22881/10882. "assessment of the role of the booster dose in breast conserwing therapy": the Dummy Run. EORTC Radiotherapy Cooperative Group. Radiother Oncol 1991:22:290-298.

24. van Tienhoven G, Mijnheer BJ, Bartelink H, Gonzalez DG. Quality assurance of the EORTC Trial 2288 1/10882: boost versus no boost in breast conserving therapy. An overview. Strahlenther Onkol 1997;173:201-207.

25. Dusserre A, Garavaglia G, Giraud JY, Bolla M. Quality assurance of the EORTC radiotherapy trial 22863 for prostatic cancer: the dummy run. Radiother Oncol 1995;36:220-234.

26. Dawis JB, Reiner B, Dusserre A, Giraud JY, Bolla M. Quality Assurance of the EORTC trial 229 11. A phase III study of post-operative external radiotherapy in pathological stage T3NO prostatic carcinoma: The durnny run. Radiother Oncol 2002;64:65-73.

27. Kouloulias VE, Bosset JF, Van Tienhoven G, Davis BJ, Piarare M, Poormans $P$, for the EORTC Radiorherapy Group. Quality assurance in the EORTC 2292』 trial on preoperative madiotherapy with or without chemotherapy for resectable rectal cancer: Evaluation of the individual case review procedure. Eur. J. Cancer 2002;38:18449-1856.

28. Valley JF, Bernier J, Tercier PA et al. Qualicy assurance of the EORTC radiotherapy trial 22931 for head and neck carcinomas: the dummy run. Radliother Oncol 1998;47:37-44.

29. Poortmans $\mathbb{P M}$, Venselaar JL, Struikmans $H$ er al. The potential impact of treament variations on the wesults of radiotherapy of the internal mammary lymph node chain: a quality-assurance report on the dummy run of EORTC Phase III randomised trial 22922/10925 in Stage I--lll breast cancer(1). Int I Radiat Oncol Biol Phys 2001:49:1399-1408.

30. Lievens Y, Poormans P, Van den Bogaert W. A glance on qualicy assurance in EORTC study 22922 evaluating rechniques for internal mammary and medial supraclavicular lymph node chain ir radiarion in breast cancer. Radiother Oncol 2001;60:257-265.

31. Poortmans P, Kouloulias VE, Venselar JL et al. Quality assurance of EORTC trial 22922/10925 investiganing the role of internal mammary - medial supraclavicular irradiation in stage I-III brease cancer: the individual case review. Eur J Cancer 2003;39: 2035-2042.

32. Bijker N, Rutgers E], Peterse JL et al. Variations in diagnostic and therapeutic procedures in at mulkicentre, randomized clinical trial (EORTC 10853) investigating breast-conserving treatment for DCIS. Eur J Surg Oncol $2001 ; 27: 135-1,40$.

33. Gomola I, Van Dam ], Tsern-Verdum ] et al. External audits of electron beams using mailed TLD dosimerry: preliminary results. Radiother Oncol 2001;58:163-168.

34. Bentzen $S M$, Bernier J, Davis JB er al. Clinical impact of dosimetry quality aseurance programmes assessed by 
radiobiological modeling of data from the therrioluminescent dosimetry study of the European Organization for Restearch and Treatment of Cancer. Eur J Cancer 2000;36:615-620.

35. Thwaitcs D, Scalliet $P$, Leer JW, Overgaind J. QA in radiotherapy; ESTRO report 1994 Radiother Oncol $1995 ; 35: 61-73$. 


\section{CHAPTER 11}

Quality Assurance in clinical trials in breast cancer: Summary and future prospects. 
The work presented in this thesis was initiated through the participation in the large multi-centre trial of the European Organisation for Research and Treatment of Cancer investigating the value of an additional boost on the primary tumour bed in breast conserving treatment for breast cancer. This has led to the involvement of the author in the Quality Assurance programme of the EORTC Radiotherapy Group, starting with the subsequent trial in breast cancer, investigating the value of regional irradiation.

Chapter 2 describes the evolution of 20 years of Quality Assurance in the EORTC Radiotherapy Group. Initially, thanks to the financial support of the European Union, a number of on-site visits to verify the physical characteristics of the treatment machines as well as the clinical parameters of trial participation were undertaken by a team of clinicians and physicists. Based on this experience, items of importance for Quality Assurance in daily practice and especially in the framework of clinical trials could be defined. This led to the definition of a number of minimum requirements to which a participating institute had to comply. Moreover, it also stimulated the collaboration within the Radiotherapy community at the European level. Thanks to this pioneering work, the "Vlaamse Kankerliga" took the initiative to support a fellowship in Quality Assurance in Radiotherapy, which was named after the late Emmanuel van der Schueren to commemorate his contribution to this work. This investment led to the completion of a number of on-going projects and to several new initiatives in the field of Quality Assurance, supervised by the study co-ordinators and by the Quality Assurance committee.

The results of the EORTC "boost-no boost trial" are often referred to because of the huge number of patients (5569), the positive outcome of the primary endpoint and the high quality of the trial. In chapter 3 the analysis after a median follow-up of 5.1 years is described. This confirmed the value of a higher boost dose to the primary tumour bed for patients up to the age of 50 with early stage breast cancer after a microscopically complete lumpectomy. Above this age, the results were already excellent without a boost dose and a definitive conclusion on its value will only be possible after a longer followup. This analysis is planned for the near future. For patients younger than 50 , a further increase in the boost dose is currently being investigated in a new Dutch trial, which is also open for participants from other countries. The prognostic value of gene and protein analysis will be investigated as a translational side study for the patients participating to the study. Chapter 4 shows, in the same prospective EORTC "boost-no boost trial", that the technique used to deliver the boost dose did not have a significant influence on the results. In the framework of this trial, a dedicated and comprehensive Quality Assurance programme was organised. Because it was the first time that Quality Assurance was 
organised in such a comprehensive way, it helped the writing of guidelines in future rrials and served as an example for later initiatives. The basic evaluation case report form developed for the Quality Assurance in this trial still remains the basis on which evaluation forms for current trials are prepared. While most of this work was published earlier, a comparison between the individual case review performed early in the trial and repeated in a number of large centres at the end of the trial was never performed. This comparison, of a kind that has never been published before, showed that the compliance to treatment improved over time for the group of institutes which participated twice to the individual case review. These results therefore suggest a positive effect of trial participation possibly mediated through the Quality Assurance programme linked to the trial. The same conclusion might be drawn from the very constant rate of local recurrences amongst the participating institutes, in contrast to the high variance in an older EORTC trial (Figures 1 and 2 of Chapter 1 of this thesis).

The role of Radiotherapy in the treatment of regional lymph nodes has been the subject of a debate since the early seventies of last century. The advocates of radiorherapy emphasised the possibility of a group of patients with microscopic tumour spread to the lymph nodes only, without distant metastases, and therefore curable with Radiotherapy, while other oncologists contested the existence of such a group and therefore emphasised the possible negative influence of irradiation on survival. This could be referring to an increase in cardiovascular mortality, especially with older treatment techniques. "To definitively end this controversy, the EORTC Radiotherapy and Breast Groups designed a trial investigating the value of internal mammary and medial supra-clavicular lymph node irradiation. With the experience of the Quality Assurance in the "boost-no boost trial", meetings were held to prepare a comprehensive Quality Assurance programme in the framework of the new trial, before finalising the protocol. In close co-operation with the study co-ordinators, the author of this thesis organised and led the entire programme. This work is summarised in chapters 6 to 9.

To conclude, in chapter 10 a number of recent developments are described and a glance into the future is taken. More than ever, Quality Assurance will continue to be a matter of close co-operation between radiation oncologists, physicists, technologists, data managers and others who are involved in the field of radiation oncology and in the multidisciplinary approach of oncology health care. 


\section{Future prospects, treats and recommendations:}

Over the last decades, significant progress has been made in the general knowledge, detection and treatment of cancer. It is becoming more and more likely that there will be no simple "solution of the cancer problem", but that we will continue for many years on the same path of progressing by steps, usually with very small steps indeed. Especially in small improvements, the final clinical effect may be blurred by the variance in patient, tumour and treatment related parameters. Therefore, the role of Quality Assurance in every aspect of cancer treatment in daily practice as well as in clinical trials will continue to be crucial to safeguard the translation of new findings and improvements into daily patient care. Much of the progress will undoubtedly be obtained with well designed clinical research, sometimes leading to large prospective trials and sometimes to more limited initiatives including translational research components. The results of the work described in this thesis clearly support the suggestion of a positive effect of trial participation, which is possibly mediated by the Quality Assurance programme linked to the trial. Therefore, trial participation, even in non-academic institutions, should be encouraged, despite the extra resources needed. Proper funding of these extra costs will have a positive impact on patient care, not only for the patients who participate to a clinical trial but also for the entire Radiotherapy department.

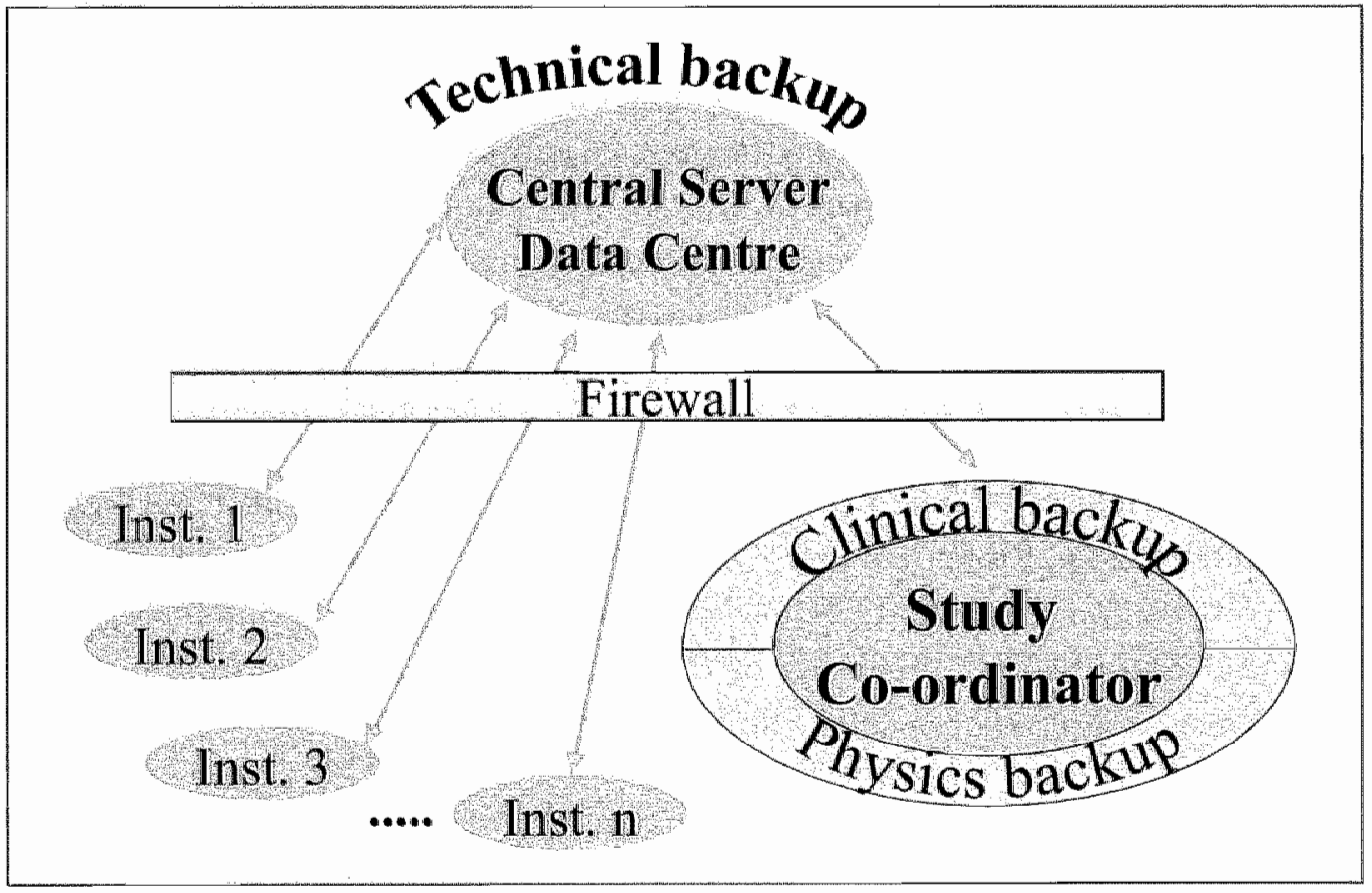

Figure 1. Simplified sobematic representation of a sytem for remote revieuing and Quality Assurance in a multi-centre setting. 
The complexity of the process of Radiotherapy continues to grow to an extent that the evaluation of the numerous steps in the treatment of the cancer patient requires the input of highly skilled scientists as well as an increasing amount of sophisticared and dedicated equipment. With the use of the new developments in the field of computer and communication technology, we are currently investigating the implementation of Quality Assurance procedures using the worldwide web (Figure 1).

Because of the infrastructure required for this purpose and since there is no absolute need to re-invent the wheel, the EORTC Radiotherapy Group is negotiating with a recently founded US-based consortium. If we can bring this co-operation to a good end, it will enable us to perform the evaluation of various patient, tumour and treatment related variables remotely and later probably interactively using our own computers, without the need for mailing data on paper or on CD-ROM or for visiting the participating departments. Especially in the start-up phase however, this requires a huge amount of work and quite some investments in infrastructure because of security reasons and because many of the facilities in use in the various Radiotherapy departments do not fully support the exchange of the data. In this respect, it was striking to see that even DICOMRT, the so-called common language for images and data in Radiotherapy, is still at present not capable to support the mutual exchange of data by many treatment planning systems and other machines like simulators.

Within the European Society for Therapeutic Radiology and Oncology (ESTRO), the ESTRO Quality Assurance Laboratory (EQUAL) gathered a huge experience in physical beam measurements. All Radiotherapy departments participating in clinical trials of the EORTC Radiotherapy Group have to organise an annual EQUAL external dosimetric audit for their treatment machines. Until recently, this was free of charge thanks to a grant from the European Commission but today the participants thave to pay for this audit. Some years ago, ESTRO has set up the European Institute for Quality Assurance in Radiotherapy (EQART), a structure to co-ordinate all aspects of QA in Radiotherapy delivery in Europe. In the early phase, the EOR'TC Radiotherapy Group discussed and evaluated the possible collaboration in the field of Quality Assurance in clinical Radiotherapy trials (figure 4). Already then, based on real cost-estimates of the infrastructure and the personnel, an important fee to be paid per trial and per patient was calculated. A grant application to the European Commission to support EQART financially was not successful. Thereafter, this activity had to be stopped but a private initiative emerged - where several aspects of Quality Assurance can be hired for a fee.

Today Quality Assurance activities impose a heavy burden on the workload of clinical oncology departments and even more on clinical trial organisations such as the EORTC. 


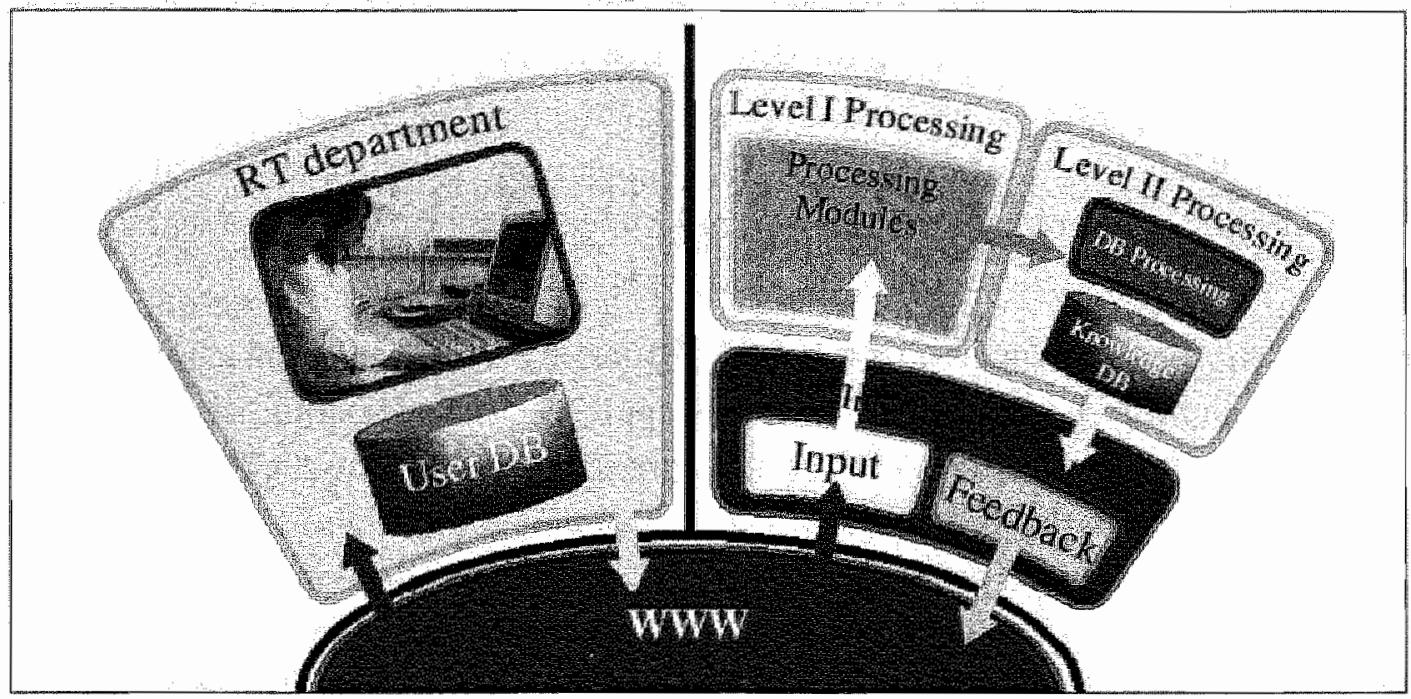

Figure 2: Schenatic representation of the web-based approath for Quality Assutance as designed by EQART (Donninique Huyskens et al).

Most if not all of this work is currently done by volunteers and by fellows in the framework of grants provided by cancer leagues. With the changing ideas on time management in the younger generations of health care workers and the growing complexity of the procedures needed to evaluate all aspects of the treatment and of the clinical and basic scientific research, we will soon need to evaluate the organisation and the financial support for the organisation of Quality Assurance. Unless it can be incorporated as a standard and essential part of the organisation and conduct of clinical trials including the reservation of sufficient financial and technical support, it might well be completely disregarded because of the lack of volunteers, money and means.

If we manage to incorporate the aspects of Quality Assurance in all parts of basic and translational research, clinical trials and routine patient treatment, it will prove to be a very cost-efficient means to swiftly recognise and translate new findings and procedures from research to bedside and to guaranty the correct implementation of improvements in the daily care of our patients. 


\section{SAMENVATTING}

Borstkanker is in de Westerse geindustrialiseerde wereld de meest voorkomende tumor bij de vrouw. Terwijl het aantal patiënten met borstkanker ten gevolge van de vergrijzing en de veranderde gezinsplanning gedurende de laatste decennia fors gestegen is neemt de mortaliteit gelukkig geleidelijk af. Daar waar tot ongeveer 15 jaar geleden nog ruim 1 vrouw op 3 die aan borstkanker leed er ook aan stierf verwachten we dat dit tegen 2015 nog slechts ongeveer 1 op 5 zal zijn. Een vroegere diagnosestelling dankzij bevolkingsonderzoek en het bewustwordingsproces bij de bevolking verklaart een deel van deze verbetering. Daarnaast heeft ook gericht klinisch wetenschappelijk onderzoek een belangrijke bijdrage geleverd door het introduceren van nieuwe behandelingen en het ondersteunen van richtlijnvorming. Deze verbetering zien we niet alleen op het gebied van de genezingskans maar ook op het gebied van de levenskwaliteit zoals bij de borstsparende behandeling en bij de schildwachtklierprocedure.

De EORTC Radiotherapie Groep was en is een pionier op het gebied van de kwaliteitscontrole bij klinische studies. Een overzicht van dit werk vormt hoofdstuk 2 van dit proefschrift. Het begon met het bezoeken van deelnemende afdelingen en kreeg later meer vorm door het vastleggen van procedures met o.a. minimum vereisten voor de apparatuur en de bestaffing van deelnemende centra, fysische metingen van de bestralingsapparatuur en met studiespecifieke programmas zoals "dummy-runs" en de individuele beoordeling van de medische dossiers van deelnemende patiënten,.

In hoofdstukken 3, 4 en 5 worden de resultaten van de EORTC studie 22881/10882 beschreven waarbij werd aangetoond dat, vooral bij jongere patiënten, het geven van een bijkomende (boost) dosis na de chirurgische verwijdering van de primaire tumor en de bestraling van de volledige borst de kans op een recidief significant vermindert. Een verdere analyse van de verschillende technieken die gebruikt werden om de boost toe te dienen toont aan dat er geen significante verschillen worden aangetroffen in de resultaten maar ook dat er een grote variatie in het gebruik van deze technieken aanwezig is naargelang de verschillende tijdsperiodes en de verschillende deelnemende landen. Deze studie was de eerste EORTC studie waarbij bij de start een uitgebreid en volledig programma van kwaliteitscontrole was voorzien. In het kader daarvan werden dossiers beoordeeld van deelnemende patiënten van vrijwel alle deelnemende instituten. Deze procedure werd bij 9 grotere deelnemers op het einde van de behandeling herhaald. Deze gegevens, die het ook mogelijk maakten om eenzelfde kwaliteitscontroleprocedure die zowel vroeg als laat in een studie werd uitgevoerd met elkaar te kunnen vergelijken, werden nooit volledig geanalyseerd en gepubliceerd. De resultaten tonen aan dat vooral 
die deelnemers waarbij aanvankelijk vrij veel afwijkingen werden vastgesteld, het bij een latere kwaliteitscontrole beter doen.

Toen de EORTC Radiotherapie en Borst Groepen in 1992 toestemming kregen om een onderzoek naar de waarde van radiotherapie van de parasternale en de mediaal supraclaviculaire lymfeklierregios op te starten werd gebruik gemaakt van de ervaring die met de kwaliteitscontrole in de boost-studie werd opgedaan. Tijdens de voorbereidende fase van de studie werd een volledig programma uitgewerkt dat was aangepast aan de verwachte specifieke eisen en verwachtingen. Hoofdstukken 6 tot en met 9 zijn een weergave van dit onderzoek, dat gecoördineerd werd door de auteur van dit proefschrift. In hoofdstukken 6 en 7 wordt de "dummy-run" beschreven waarvoor deelnemende instituten werden gevraagd om de bestralingsplanning wit te werken op een "papieren patiënt". In hoofdstuk 8 wordt de evaluatie van de beoordeling van de medische gegevens van in totaal 6 patiënten per deelnemend instituut weergegeven. Hierbij werden naast de bestralingstechnieken ook een aantal patiënt en tumorgebonden parameters beoordeeld. In hoofdstuk 9 tenslotte worden de resultaten van de "dummy-run" en de beoordeling van de gegevens van de deelnemende patiënten per instituut met elkaar vergeleken voor wat betreft de bestralingstechnieken en de daarmee berekende dosis ter hoogte van het parasternale gebied. Hieruir blijkt dat met de "dummy-run" reeds veel informatie kan bekomen worden over de gebruikte bestralingstechnieken en over mogelijke onduidelijkheden in het studieprotocol. De beoordeling van de echte patiënten laat evenwel niet alleen de beoordeling van patiënt en tumorgebonden parameters toe maar daarnaast ook de evaluatie van specifieke aspecten van de bestralingsbehandeling zoals het gebruik van beeldvorming (bijv. CT-scan) voor het aanpassen van de radiotherapie an de anatomie van de individuele patiënt. Uit deze vergelijking blijkt tenslotte dat de behandeling bij de echte patiënten beter overeenkomt met de gewenste behandeling zoals voorgeschreven door het studieprotocol.

Kwaliteitscontrole dient zich aan te passen aan de gebruikte middelen en procedures waaronder de steeds complexer wordende radiotherapie technieken. Dankzij de steeds voortschrijdende ontwikkelingen op het gebied van beeldverwerking en de verzending van gegevens zal het in de nabije toekomst mogelijk worden om ook voor de kwaliteitscontrole, zowel binnen als buiten het kader van een klinische trial, geheel nieuwe procedures inclusief het gebruik van het wereldwijd internet aan te wenden. Bovendien wordt kwaliteitscontrole meer en meer gezien als een zaak van alle betrokkenen bij de multidisciplinaire oncologische behandeling. Hoofdstuk 10 is in het kader van deze beide aspecten geschreven als bijdrage van de radiotherapic aan een speciaal nummer van het Europese tijdschrift voor chirurgische oncologie. 


\section{CURRICULUM VITAE}

\section{POORTMANS Philip, Martin, Peter}

Born: May 31, 1961 Herentals (Belgium)

Dr. Bernard Verbeeten Instiruut

PO Box 90120

Private: Kennedylaan?

5000 LA Tilburg

The Netherlands 2200 Herentals

Tel: **31-(0) 13-5947777

Belgium

Tel: **-31-(0)13-5947765 (direc)

Tel: ${ }^{* *}-32-(0) 14-215193$

Fax: ${ }^{* *}-31-(0) 13-5947683$

e-mail: poortmans.ph@bvi.nl

\section{Studies and training:}

School:

1973-1979:

Gemeenschapsonderwijs Herentals and Turnhout, Latin-Mathematics

University: 1979-1982:

Rijks Universitair Centrum Antwerpen, cum laude.

1982-1986:

Universitaire Instellingen Antwerpen, cum magna laude

Medical degree: Antwerp, Belgium, 10 juli 1986

Training: $\quad$ AZ Middelheim, Antwerp, Prof. Dr. W. Van den Bogaert

St.-Vincentiushospital, Antwerp, Dr. G. Langerock

AZ Middelheim, Antwerp, Prof. Dr. P. Scalliet

18-04-1991: Registration radiation oncologist, Belgium

16-11-1991: Registration radiarion oncologist, The Netherlands

\section{Professional:}

$01-10-90-30-06-91$ :

St.-Elisabethziekenhuis, Turnhout, Belgium

since 01-07-91:

Dr. Bernard Verbeeten Instituut, Tilburg, The Necherlands

\section{Memberships:}

- B.V.R.O. (Belgische Vereniging voor Radiotherapie en Oncologie)

- N.V.R.O. (Nederlandse Vereniging voor Radiotherapie en Oncologie)

- E. S.T.R.O. (European Society for Therapeutic Radiology and Oncology)

- E.O.R.T.C. (European Organisation for Research and "Treatment of Cancer) Radiotherapy Group

- E.O.R.T.C. Lymphoma Group

\section{Current scientific responsabilities:}

- Member Steening Commitree E.O.R.T.C. Radiotherapy Group.

- "Quality Assurance co-ordinator" of the E.O.R.T.C. Radiotherapy Group.

- Member "Radiorherapy subcommittee" of the E.O.R.T.C. Lymphoma Group.

- Member C.K.T.O. (Dutch advisory board on clinical research).

- Member "Editorial Board" "Trialnieuws".

- Member "Editorial Board" "Radiotherapy \& Oncology".

- Member "Shadow Scientific Committee" 13th bi-annual ECCO meeting, Paris, 2005. 


\section{PUBLICATIONS}

\section{Quality assurance:}

- Poortmans, Philip M.P.; Venselaar, Jack L.M.; Struikmans, Henk; Hurkmans, Coen W; Davis, J. Bernard; Huyskens, Dominique; van Tienhoven, Geertjan, MD; Vlaun, Vernon; Lagendijk, Jan J.W.; Mijnheer, Ben J.; De Winter, Karin A.; Van der Hulst, Marleen H.; Van den Bogaert, Walter F.C. The potential impact of treatment variations on the results of radiotherapy of the internal mammary lymph node chain: A qualiry-assurance report on the dummy run of EORTC phase III randomized trial 22922/10925 in stage II-III breast cancer. Int. J. Radiat. Oncol. Biol. Phys. 49: 1399-1408, 2001.

- Jacques Bernier, Jean-Claude Horiot, Harry Bartelink, Karl-Axel Johansson, Luca Cionini, Dionisio Gonzalez Gonzalez, Walter Van den Bogaert, Philip Poortmans, Bernard Davis, Frances Godson, on behalf of the Radiotherapy Cooperative Group of the European Organization for Research and Treatment of Cancer. Minimum requirements for infrastructure, staff and workload in radiotherapy departments contriburing to the EORTC clinical activities. Work-document of the EORTC, August 2001.

- Bernier J, Horior JC, Poortmans P. Quality assurance in radiorherapy: from radiation physics to patient- and trial-oriented control procedures. Eur. J. Cancer 38 (Suppl. 4: ESSM 26-28 March): S155-8, 2002.

- Vassilis E. Kouloulias, Jean-Francois Bosser, Geertjan Van Tienhoven, Bernard J. Davis, Marianne Pierart, Philip Poortmans, for the EORTC Radiotherapy Group. Quality assurance in the EORTC 22921 trial on preoperative radiotherapy with or without chemotherapy for resectable rectal cancer: Evaluation of the individual case review procedure. Eur. J. Cancer 38: 1849-1856, 2002.

- Yolande Lievens, Philip Poortmans, Walter Van den Bogaert. A glance on quality assurance in EORTC study 22922 evaluating techniques for internal mammary and medial supraclavicular lymph node chain irradiation in breast cancer. Radiother. Oncol. 60:257-265, 2001.

- Monograph: J. Berridge, M. Coffey, G. Brusadin, M. Ramalho, W. Sapru, V. Vlaun, G. Vandevelde, M. Pierart, J. Bernier, P. Poortmans, on behalf of the EORTC radiotherapy technologists group. Guidelines for radiotherapy technologists in the management of dinical trials involving radiotherapy. EORTC Radiotherapy Technologists Group Publication, Brussels, December 2002.

- Vassilis E. Kouloulias, Philip M. Poortmans, Jacques Bernier, Jean-Claude Horior, Karl-Axel Johansson, Bernard Davis, Frances Godson, Guido Garavaglia, Marianne Pierart, Emmanuel Van der Schueren. The quality assurance programme of the Radiotherapy Group of the European Organization for Research and Treatment of Cancer (EORTC): a critical appraisal of 20 years of continuous efforts. Eur J Cancer 39: 430-437, 2003.

- Kouloulias VE, Ntasis E, Poortmans P, Maniaris TA, Nikita KS. A scenario for a web-based radiation treatment planning structure: A new tool for quality assurance procedure? Technol Health Care: 11(2):105-114, 2003.

- P. Poortmans, V.E. Kouloulias, J.L. Venselaar, H. Struikmans, J.B. Davis, D. Huyskens, G. van Tienhoven, C. Hurkmans, B. Mijnheer, W. Van den Bogaert. Quality assurance of EORTC trial 22922/10925 investigating the role of internal mammary - medial supraclavicular irradiation in stage I-III breast cancer: the individual case review. Eur J Cancer 
39: 2035-2042, 2003.

- Kouloulias VE, Poortmans P, Antypas C, Kappas C, Sandilos P. Field Hatness and symmetry of photon beams: Review of the current recommendations. Technol Health Care 11 (4): 283$288,2003$.

- Ataman F, Poortmans P, Stupp R, Fisher B, Mirimanoff RO. Quality assurance of the EORTC 26981/22981; NCIC CE3 intergroup trial on radiotherapy with or withour temozolomide for newly-diagnosed glioblastoma multiforme: the individual case review. Eur I Cancer 40:1724-30, 2004.

- Ataman F, Poormans P, Davis JB, Bernier J, Giraud JY, Kouloulias VE, Pierart M, Bolla M. High conformality radiotherapy in Europe: thirty-one centres participating in the quality assurance programme of the EORTC prostate trial 22991. Eur J Cancer 40: 2411-2416, 2004

- Philip M. Poortmans, J. Bernard Davis, Fatma Ataman, Jacques Bernier, Jean-Claude Horiot, for the EORTC Radiotherapy Group. The Quality Assurance Programme of the Radiotherapy Group of the European Organisation for Research and Treatment of Cancer: Past, Present and Future. Eur J Surg Oncol, accepted, 2004.

- Philip Poortmans, Fatma Ataman, J. Bernard Davis, Harry Bartelink, Jean-Claude Horiot, Marianne Pierart, Laurence Collette, Geertjan Van Tienhoven. Quality Assurance in the EORTC phase III randomised 'boost vs. no boost' trial for breast conserving therapy: comparison of the results of two individual case reviews performed early and late during the accrual period. Radiother Oncol Accepted, April 2005.

- Philip Poortmans, Philippe Lambin, Vassilis Kouloulias, Laurence Collette, Geertjan van Tienhoven, Henk Struikmans, Jack L. Venselaar, Walter Van den Bogaert, J. Bernard Davis, on behalf of the EORTC Radiotherapy and Breast Cancer Groups. Qualiry assurance in the EORTC randomised trial 22922/10925 investigating irradiation of the internal mammary and medial supraclavicular lymph node chain works. Eur J Cancer submitted, 2004.

- Berthe M.P. AJeman, Théodore Girinsky, Richard W.M. van der Maazen, Simon Strijk, Paul Meijnders, Roberto Bortolus, Manouk J.J. Olofsen-van Acht, Marnix L.M. Lybeert, Yolande Lievens, Houchingue Eghbali Evert M. Noordijk, Radka Tom_i, Jacobus H. Meerwaldt, Philip M.P. Poortmans, Wilma G.J.M. Smit, Antonella Pinna, Michel Henry-Amar, John M.M. Raemaekers, on behalf of the European Organization for Research and Treatment of Cancer (EORTC) Lymphoma Group.Quality control of involved-field radiotherapy in patients with advanced Hodgkin's lymphoma (EORTC 20884). Int. J. Radiat. Oncol. Biol. Phys. Accepted, March 2005.

\section{Breast cancer:}

- C. Vrieling, J.C. Horiot, E. Van der Schueren, P. Poortmans, H. Struikmans, L. Collette, M. Pierart, H. Bartelink. Is a boost dose essential in breast conserving therapy? An EORTC phase III study (22881/10882). Proceedings 8th Varian European Users Meeting, Vilamoura, Portugal, April 27-30, 1997, pp 8-10, 1998.

- A.-M. Ptaszynski, W. Van den Bogaert, J.-C. Horiot, Ph. Poortmans, A. Fourquet, H. Struikmans, H. Bartelink, M. Pierart, L. Collette, E. van der Schueren: Radiation dose homogeneity in an EORTC multicenter trial on breast irradiation. Acta Oncologica, vol. 38, suppl. 13, pp. 19-27, 1999. 
- C. Vrieling, L. Collette, E. Bartelink, J.H. Borger, S.J. Brenninkmeyer, J.-C. Horiot, M. Pierart, Ph.M. Poortmans, H. Struikmans, E. Van der Schueren, J.A. Van Dongen, E. Van Limbergen, H. Bartelink. On behalf of the EORTC Radiotherapy and Breast Cancer Cooperative Groups. Validation of the methods of cosmetic assessment after breastconserving therapy in the EORTC "boost versus no boost" trial. Int. J. Radiat. Oncol. Biol. Phys. 45: 667-676, 1999.

- C. Vrieling, L. Collette, A. Fourquet, W.J. Hoogenraad, J.-C. Horiot, J.J. Jager, M. Pierart, Ph.M. Poortmans, H. Struikmans, M. Van der Hulst, E. Van der Schueren, H. Bartelink. On behalf of the EORTC Radiotherapy and Breast Cancer Cooperative Groups. The influence of the boost in breast-conserving therapy on cosmetic outcome in the EORTC "boost versus no boost" trial. Int. J. Radiat. Oncol. Biol. Phys. 45: 677-685, 1999.

- Conny Vrieling, Laurence Collette, Alain Fourquet, Willem J. Hoogenraad, Jean-Claude Horiot, Jos J. Jager, Marianne Pierart, Philip M. Poortmans, Henk Struikmans, Ben Maat, Erik van Limbergen, Harry Bartelink. On behalf of the EORTC Radiotherapy and Breast Cancer Cooperative Groups: The influence of patient, tumor and treatment factors on the cosmetic results after breast-concerving therapy in the EORTC "boost vs. no boost" trial. Radiother. Oncol. 55: 219-232, 2000.

- Ph. Poortmans. Nabestraling na borstsparende operatie halveert antal lokale recidieven: eerste resultaten van grote EORTC-trial zijn bemoedigend. Kanker 24: 18-21, 2000.

- C. Vrieling, L. Colletre, A. Fourquet, W.J. Hoogenraad, J.C. Horiot, J.J. Jager, S. Bing Oei, H.L. Peterse, M. Pierart, P.M. Poortmans, H. Struikmans, W. Van den Bogaert, H. Bartelink, on behalf of the EORTC Radiotherapy, Breast Cancer Groups. Age related to treatment, parhology and local control in breast-conserving therapy. Thesis Free University, Amsterdam, The Netherlands, 87-109, 2001.

- Ph. Poortmans. Nabestraling na borstsparende operatie halveerr antal. lokale recidieven: eerste resultaten van grote EORTC-trial zijn bemoedigend. Gamma 5: 2-5, 2001.

- Harry Bartelink, Jean-Claude. Horiot, Philip Poortmans, Henk Struilkmans, Walter Van den Bogacr, Isabelle Barillor, Alain Fourquer, Jacques Borger, Jos Jager, Willem Hoogenraad, Laurence Collecte, Marianne Pierart. Recurrence rates after treatment of breast cancer with standard radiotherapy with or without additional radiation. N. Engl. J. Med. 345: 1378$1387,2001$.

- Bartelink H, Poortmans PhMP, Struikmans $\mathrm{H}_{n}$ Borger JH, Jager JJ, Hoogenraad WJ Van den Bogaert W, Barillot I, Fourquer A, Coletre L, Pierart M en Horiot J-C. Minder lokale recidieven van borstkanker door extra lokale bestralingsdosis na mammasparende chirurgie; 5jaarsresultaten van een klinische trial. Ned. T. Geneesk. 146: 416-423, 2002.

- Bartelink Harry, Horior Jean-Claude, Poormans Phillip. Correspondence, reply of the aurhors: Radiotherapy for breast cancer. N. Engl. J. Med. 346: 863-864, 2002.

- Philip Poortmans. Lymfoedeem: de invloed van de behandeling van borstkanker. Oedeminus 5: 8-14, 2002.

- Bartelink H., Poortmans Ph.M.P., Struikmans H. Antwoord van de autheurs op correspondentie van G. van Tienhoven "Is bij borstkankerpatiënten ouder dan 60 jaar na een mammasparende operatie extra lokale bestraling nog wel geïndiceerd?" Ned. T. Geneesk. 146: $824,2002$.

- D. van Loon, L.L. Maas, P. Tobé, F.E.M. de Vries, Ph. Poortmans, P.H. van der Giessen, E. Raaijmakers. Huidreacties bij thoraxwandbestralingen: een evaluatie. Gamma 2: 2-6, 2003. 
- C. Vrieling, L. Colletre, A. Fourquet, W.J. Hoogenraad, J.C. Horiot, J.J. Jager, S. Bing Oei, H.L. Peterse, M. Pierart, P.M. Poortmans, H. Struikmans, W. Van den Bogaert, H. Bartelink, on behalf of the EORTC Radiotherapy, Breast Cancer Groups. Can patient-, treatment- and pathology-related characteristics explain the high local recurtence rate following breastconserving therapy in young patients? Eur J Cancer 39 (7): 932-944, 2003.

- M.F.Ernst, A.C.Voogd, J.W.W.Coebergh, P.M.Poortmans, J.A.Roukema. Using loco-regional recurrence as an indicator of the quality of breast cancer treatment. Eur J Cancer $40: 487-493$, 2004. (+ editorial)

- Philip Poortmans, Harry Bartelink, Jean-Claude Horiot, Henk Struikmans, Walter Van den Bogaert, Alain Fourquet, Jos Jager, Willem Hoogenraad, Patrick Rodrigus, Carla WárlámRodenhuis, Laurence Collette, Marianne Pierart, on behalf of the EORTC Radiotherapy and Breast Cancer Groups. The influence of the boost technique on local control in breast conserving treatment in the EORTC "boost versus no boost" randomised trial. Radiother Oncol 72:25-33, 2004.

- Ninja Antonini, Heather Jones, Jean Claude Horior, Philip Poortmans, Henk Struikmans, Walter van den Bogaert, Isabelle Barillot, Alain Fourquet, Jos Jager, Willem Hoogentaad, Laurence Collette, Marianne Pierart, Guus Hart, Harry Bartelink. Relation of local control with age and radiation dose after breast conserving treatment in the randomised EORTC Trial 22881/10882. Ready to be submitted, April 2005.

\section{Malignant lymphoma:}

- Philip Poortmans. More than 50 years of evolution in radiotherapy in Hodgkin's disease: from generalisation to individualisation. Proceedings of the Russian-Dutch conference "Diagnosis and treatment of lymphomas." Sint-Petersburg, 26-27 september 2002.

- Philip Poortmans. Primary central nervous system lymphoma: a multidisciplinary challenge! Proceedings of the Russian-Dutch conference "Diagnosis and treatment of lymphomas." SintPetersburg, 26-27 september 2002.

- Sylvie Martiner, Mahmur Ozsahin, Yazid Belkacémi, Christine Landmann, Philip Poortmans, Christoph Oehlere, Luciano Scandolaro, Marco Krengli, Philippe Maingon, Raymond Miralbell, Gabriela Studer, Bruno Chauvet, Simone Marnitz, Abderrahim Zouhair, RenéOlivier Mirimanoff. Outcome and prognostic factors in orbital lymphoma: a rare cancer network study on 90 consecutive patients treated with radiotherapy. Int. J. Radiat. Oncol. Biol. Phys. 55: 892-898, 2003.

- Phillip Poortmans. Chlorambucil of twee maal bestralen? Onderzoek bij laaggradige NonHodgkin lymfoom. Regionaal Trialnieuws 7:6, maart 2003.

- Philip M.P. Poortmans. Primary central nervous system lymphoma: a continuing therapeutic challenge. Cancerfutures 2: 76-82, 2003.

- R.L.M. Haas, Ph. Poortmans, D. de Jong, B.M.P. Aleman, L.G.H. Dewit, M. Verheij, A.A.M. Hart, M.H.J. van Oers, M. van der Hulst, J.W. Baars, and H. Bartelink. High response rates and lasting remissions after low-dose involved field radiotherapy in indolent lymphomas. J Clin Oncol 21: 2474-2480, 2003.

- Philip M.P. Poortmans, Hanneke C. Kluin-Nelemans, Hanny Haaxma-Reiche, Mars Van't Veer, Mads Hansen, Pierre Soubeyran, Martin Taphoorn, José Thomas, Martin Van den Bent, Martin Fickers, Gustaaf Van Imhoff, Cynthia Rozewicz, Ivana Teodorovic, and Martine van 
Glabbeke. High-dose methotrexate-based chemotherapy followed by consolidaring radiotherapy in non-AIDS-related primary central nerwous system lymphoma: European Organization for Research and Treatment of Cancer Lymphoma Group phase II trial 20962. J Clin Oncol 21: 4483-4488, 2003. (+ editorial)

- H. Harder, H. Holtel, J. E.C. Bromberg, P. Poormans, H. Haaxma-Reiche, H. C. KluinNelemans, J. Menten and M. J. vari den Bent. Cognitive status and quality of life after treatment for primary CNS lymphoma. Neurology 62:544-547, 2004. (+ editorial)

- Philip M.P. Poortmans. Primary Central Nervous System Lymphoma: a case report of a curable brain tumor. Am J Oncol Rev 3:386-390, 2004.

- Shenkier TN, Blay JY, O'Neill BP, Poortmans P, Thiel E, Jahnke K, Abrey LE, Neuwelt E, Tsang R, Batchelor 'T, Harris N, Ferreri AJ, Ponzoni M, O'Brien P, Rubenstein J, Connors JM. Primary CNS lymphoma of T-cell origin: a descriptive analysis from the international primary CNS lymphoma collaborative group. J Clin Oncol 23:2233-2239, 2005.

- R.L.M. Haas, Ph. Poortmans, D. de Jong, M. Verheij, M. van der Hulst, J.P. de Boer, H. Bartelink. Effecrive palliation by low dose local radiotherapy of $4 \mathrm{~Gy}$ for recurrent and/or chemotherapy refractory non-follicular lymphoma patients. Eur J Cancer, accepred, April 2005.

- Lauren E. Abrey, Tracy T. Barchelor, Andres M. Ferreri, Mary Gospodarowicz, Elisa J. Pullczynski, Emanuele Zucca, Justine Smith, Agnieszka Korfel, Carole Soussain, Lisa M. DeAngelis, Edward A. Neuwelt, Brian P. O'Neill, Eckhard Thiel, Tamara Shenkier, Frasesc Graus, Martin van den Bent, John F. Seymour, Philip Poortmans, James O. Armitage and Franco Cavalli for the International Primary CNS Lymphoma Collaborative Group (IPCG). Report of an International Workshop to Standardize Baseline Evaluation and Response Criteria for Primary Central Nervous System Lymphoma. J Clin Oncol, resubmitted after minor comments, March 2005.

- Mahmut Ozsahin, Richard W. Tsang, Philip Poortmans, Yazid Belkacémi, Michel Bolla, Fazilet Öner Dinçbas, Chrisține Landmann, Bernard Castelain, Jeroen Buijsen, Jürgen Curschmann, Sidney P. Kadish, Anna Kowalczyk, Yavuz Anacak, Josef Hammer, Tan D. Nguyen, Gabriela Studer, Rachel Cooper, Meriç Sengöz, Luciano Scandolaro, and Abderrahim Zouhair. Outcome and patterns of failure in solitary plasmacytoma: A mulricenter Rare Cancer Network study of 258 patients. Blood, submitted, 2005.

- Maggie Klessens, Linda van Rijen, Richelle Reijenga, Sandra Hol, Marjan van de Pol, Philip Poortmans. Bestralingstechnieken voor mediastinaal gelokaliseerde maligne lymfomen. Gamma, ingediend, april 2005.

\section{Miscellaneous:}

- De invloed van kanker en zijn behandeling op her seksuele leven bij de mens. Ph. Poortmans, G. Van Damme. Actualiteiten, 11: 71-80, 1988.

- Ph. Poortmans, H. Hamers. Postoperative Radiation Therapy for Rectal Cancer: An Interim Analysis of a Prospective, Randomised Multicenter Trial in The Netherlands ("Letter to the editor"). Cancer 69: 3016-3019, 1992.

- Ph. Lambin, Ph. Poortmans, J. Menten, H.P. Hamers. Accelerared radiorherapy with carbogen and nicotinamide (ARCON) in high grade malignant gliomas. ("Letter to the editor") Radiotherapy \& Oncology 43: 324, 1997. 
- P.N. Post, P.J.M. Kil, A.J.M. Hendrikx, Ph.M.P. Poormans, M.A. Crommelin, J.-W. Coebergh. On behalf of the regional study group for urological oncology IKZ, Eindhoven: Trend and variation in treatment of localized prostate cancer in the southern part of the Netherlands, 1988-1996. European Urology 36: 175-180, 1999.

- Philip Poortmans, Ans Vulto, Esther Raaijmakers. Always on a Friday? Time pattern of referral for spinal cord compression. Acta Oncologica 40: 88-91, 2001.

- Philip Poortmans. CCMO-perikelen: Een verhaaltje over de bomen en het bos. Regionaal Trialnieuws 2:7-8, mei 2001.

- Yuen Choi Hsia, Philip Poortmans, Ans Vulto, Linda van Rijen. Motivatie voor een rookstop: Zijn pariënten die curatieve radiotherapie krijgen gemotiveerder om te stoppen met roken als rookstop gunstig is voor de behandeling? Gamma 11: 20-23, 2002.

- Yazid Belkacémi, Christine Berger, Philip Poortmans, Gaëlle Piel, Abderrahim Zouhair, JeanBaptiste Méric, Tan-Dat Nguyen, Marco Krengli, Frank Behrensmeier, Abdelkarim Allal, Dany De Looze, Jacques Bernier, Luciano Scandolaro, René-Olivier Mirimanoff, and on behalf of the Rare Cancer Nerwork. Management of primary anal canal adenocarcinoma: a large retrospective study from the Rare Cancer Network. Int. J. Radiar. Oncol. Biol. Phys. 56: 1274-1283, 2003.

- Tan Dat Nguyen, Philip MP Poortmans, Marleen van der Hulst, Gabriela Studer, Éva Pigois, Timothy D Collen, Yazid Belkacemi, Véronique Beckendorf, Raymond Miralbell, Luciano Scandolaro, Guy Soete, Salvador Villa, Eliahu Gez, Olivier Thomas, Marco Krengli, Marie Saliou. Prostate cancer diagnosed before 55: a retrospective study from the Rare Cancer Network. Bulletin du Cancer 91: 959-964, 2004.

- Dirk Rades, Lukas J.A. Stalpers, Theo Veninga, Rainer Schulte, Peter J. Hoskin, Nermina Obralic, Amira Bajrovic, Rudolf Schwarz, Maarten C. Hulshof, Philip Poortmans, and Steven E. Schild. Evaluation of five radiation schedules and prognostic factors for metastatic spinal cord compression in a series of 1304 patients. J Clin Oncol, accepted, March 2005.

- J.C.M. Vulto, W.J.Louwman, Ph. Poortmans, J.W.W. Coebergh. Hospital variation in referral for primary radiotherapy in South Netherlands, 1988-1999. Eur J Cancer, Accepted, April 2005.

- Marieke Moolenaar, Robert Louwrens Poorter, Petrus Paulus Gerardus van der Toorn, Albert Willem Lenderink, Philip Poortmans, Antonius Cornelis Gerardus Egberts. The effect of honey compared to conventional treatment on healing of radiotherapy-induced skin toxicity in breast cancer patients. Acta Oncologica, submitted, 2005.

- Tan Dat Nguyen, Philip M.P. Poortmans, Marleen van der Hulst, Gabriela Studer, Eva Pigois, Timothy D. Collen, Yazid Belkacemi, Véronique Beckendorf, Raymond Miralbell, Luciano Scandolaro, Guy Soete, Salvador Villa, Eliahu Gez, Olivier Thomas, Marco Krengli, Corinne Merle, $M$ Saliou. The curative role of radiotherapy in adenocarcinoma of the prostate in men under 55: A Rare Cancer Network study. Ready to be submitted, March 2005.

- Some more arricles are under preparation. 


\section{ACKNOWLEDGEMENTS}

First of all I would like to thank my parents and grandparents and all my teachers and professors who have helped me on the way to learn the process of continuous learning and studying. During my training, Walter Van den Bogaert, Guy Langerock, Anne Strubbe, Allan van Oosterom, Pierre Scalliet and Daniëlle van den Weyngaert teached me not just a profession but the art of Radiation Oncology: thank you sincerely for this. A word of thanks also for colleague Jacques. Rutten who not only advised me as a young medical doctor in the choice for Radiation Oncology but later also helped me on the way to Tilburg. It's a merit of the good organisation of the Dr. Bernard Verbeeten Instituut and of the excellent team of colleagues and collaborators that we - as a team - could contribute to the high standing clinical research within the framework of the EORTC. Not only the participation itself but also the high priority that you all gave to clinical trial research in the surrounding of a department with a very high patient workload have supported and facilitated my work for the EORTC. A special word of thanks to my former colleague and director Han Hamers who inspired me in this and who preceded me in the EORTC Radiotherapy Group. It is in this framework that Marleen Van der Hulst, data manager, acquired the skills to handle with the sometimes complicated case report forms. It's thanks to her strive to perfection that we have an excellent reputation at the EORTC Data Centre for the quality of reporting of data. I cannot summarise all the names of all colleagues and collaborators of the EORTC Radiotherapy Group, the EORTC Lymphoma Group and at the EORTC Data Centre to whom I own my gratitude not only for the nice co-operation but also for their friendship. I remember very well how Michel Bolla, who was chairman of the Radiotherapy Group at that time, has striven for my election in the Steering Committee. I consider the members of the Steering Committee rather as friends than as colleagues: dear Abraham, Bernard, David, Fons, Frances, Geertjan, Harry, Jacques, Jan-Willem, Jean-Claude, Jean-François, Karin, Laurence, Maria, Marie-Hélène, Mary, Michel, Philippe, Philippe, René-Olivier, RolfPeter, Salvador, Vincent, Volker, Walter, thank you for your friendship and for your kindness. Without participating investigators and motivated patients, clinical research would not be possible. Therefore, we should not forget that, even in times when things are not going as smooth as desired, clinical research should be attractive by it's design and friendly by it's management. Of course, I also thank warmly everybody else who I did not mention specifically but who invested time in the preparation or in the completion of this thesis, including the members of the evaluation and of the promotion commission and many others. 
Dear Walter, I will never forget my first months in Radiation Oncology chat I have spend under your supervision, now nearly 20 years ago. You helped me to become adult in real - professional - life and to be myself. By chance, we met each other again several years later, you as study co-ordinator and I as candidate to manage the Quality Assurance programme of your trial. This co-operation turned out to become very fruitful and forms the base of this thesis. Thank you very much for your guidance!

Dear Philippe, it was at a CERRO meeting, 3 years ago that you suggested me to combine the work I was doing in Quality Assurance into a thesis. The opportunity to present and defend this thesis under your supervision at the University of Maastricht was a unique chance to combine my daily patient work with my passion for clinical trial research and Quality Assurance. I'll keep this in mind forever!

Dear Bernard, my partner in Quality Assurance in the Radiotherapy Group. When I first met you 10 years ago, you were the mysterious physicist in the Steering Committee of the Radiotherapy Group. While learning you better, the appreciation only has grown but the mystery did not completely disappear. Thank you endlessly for what you have learned me and are still learning to me about Quality Assurance in Radiotherapy and in clinical trials. I am sure that we will share many more nice projects together.

I thank the "Vlaamse Kankerliga" for their support of the Emmanuel Van der Schueren fellowship in Quality Assurance in Radiation Oncology. With this support, I had the chance to supervise and work, together with Bernard Davis, with Vassilis Kouloulias, Fatma Ataman and now Elena Musat. Thanks to their contribution a lot of work in the field of Quality Assurance in the EORTC Radiotherapy Group could be organised and completed. I feel honoured that I had the opportunity to supervise and work together with these magnificent young colleagues.

Dear Marianne. It has always been a pleasure to work together with you, not only for your kindness and for your willingness to mail or fax me supplementary data when I needed them for yet another Quality Assurance package I received from the participating institutes, but also for your good ideas and support during meetings and discussions.

And than, last but not least, my dearest Marijike, Renee and Michelle. I feel that I should rather apologise than thank you for the freedom of time you have given me to work... for my patients, my thesis and all other activities I could not say no to ... It was often only in Spain that I still could concentrate fully on you - but even there I took often my laptop with me. Still I know it was not tolerance but real support you have given to me. Thank you infinitely for this! 
Tout ce qui est dans la limite du possible doit être et sera accompli.

Jules Verne, $1828-1905$ 